Old Dominion University

ODU Digital Commons

Physics Theses \& Dissertations

Physics

Fall 12-2020

\title{
Deeply Virtual Compton Scattering at Hall A, Jefferson Lab
}

Mohamed Nuhman Hashir Rashad

Old Dominion University, mhash005@odu.edu

Follow this and additional works at: https://digitalcommons.odu.edu/physics_etds

Part of the Elementary Particles and Fields and String Theory Commons, and the Nuclear Commons

\section{Recommended Citation}

Hashir Rashad, Mohamed N.. "Deeply Virtual Compton Scattering at Hall A, Jefferson Lab" (2020). Doctor of Philosophy (PhD), Dissertation, Physics, Old Dominion University, DOI: 10.25777/z7th-my96 https://digitalcommons.odu.edu/physics_etds/129

This Dissertation is brought to you for free and open access by the Physics at ODU Digital Commons. It has been accepted for inclusion in Physics Theses \& Dissertations by an authorized administrator of ODU Digital Commons. For more information, please contact digitalcommons@odu.edu. 


\title{
DEEPLY VIRTUAL COMPTON SCATTERING AT HALL A, JEFFERSON LAB
}

\author{
by \\ Mohamed Nuhman Hashir Rashad \\ B.Sc February 2010, University of Peradeniya \\ M.S. May 2012, Old Dominion University
}

A Dissertation Submitted to the Faculty of

Old Dominion University in Partial Fulfillment of the

Requirements for the Degree of

DOCTOR OF PHILOSOPHY

PHYSICS

OLD DOMINION UNIVERSITY

December 2020

Approved by:

Charles E. Hyde (Director)

Anatoly Radyushkin (Member)

Moskov Amaryan (Member)

Alexander Gurevich (Member)

Hani Elsayed-Ali (Member) 


\title{
ABSTRACT \\ DEEPLY VIRTUAL COMPTON SCATTERING AT HALL A, JEFFERSON LAB
}

\author{
Mohamed Nuhman Hashir Rashad \\ Old Dominion University, 2020 \\ Director: Dr. Charles E. Hyde
}

The Standard Model of particle physics defines quarks and leptons as the basic building blocks of all matter. The interaction between them are mediated by force carrying gauge bosons. Quantum ChromoDynamics (QCD), the theory that explains the strong interaction is still not complete enough to derive the physical observables of a Quark-Gluon system from the fundamental degrees of freedom of it's constituents. Experimentally observable single particle densities provide important insights into our understanding of the quark-gluon system and hence help fill in the gaps of QCD. Generalized Parton Distributions (GPDs) provide simultaneous information of both spacial and longitudinal momentum distributions of constituents of a quark-gluon system. Deeply Virtual Compton Scattering (DVCS) is understood to be the simplest and cleanest process to access GPDs. Even though the exclusive DVCS is simple to understand, the experimental process however, is complex with the Bethe-Heitler and $e+p \rightarrow e+\gamma+N \pi$ being in the mix of the electron proton scattering $e+p \rightarrow e+\gamma+X$. Over the years, 3 generations of DVCS experiments have been conducted in the Experimental Hall-A of Thomas Jefferson National Accelerator Facility (JLab). This thesis presents the extraction of DVCS cross section in 9 total kinematic points from the $3^{\text {rd }}$ generation experiment (DVCS3) conducted after the $12 \mathrm{GeV}$ upgrade of the Continuous Electron Beam Accelerator Facility (CEBAF) of JLab during Fall 2014 - Fall 2016. 
Copyright, 2021, by Mohamed Nuhman Hashir Rashad, All Rights Reserved. 


\section{ACKNOWLEDGMENTS}

As the saying goes, There is no such thing as a self-made man, We are made up of thousands of others. It is true in life and it is certainly true in scientific research. I would like to thank everyone who were a part of my success, actively or passively; visibly and invisibly.

I would like to express my sincere and deepest gratitude to my thesis advisor Prof. Charles Hyde for all his guidance and encouragement during the entire course of my $\mathrm{PhD}$ work. I'm extremely grateful for his support and help throughout the years and the freedom he gave me to work on things I'm interested in and his patience and understanding. He inspired me as an academic as well as a genuine human being and I'm extremely fortunate to have known him and worked with him.

With great pleasure, I would like to thank the rest of my thesis committee Dr. Anatoly Radyushkin, Dr. Moskov Amaryan, Dr. Alexandre Gurevich and Dr. Hani Elsayed-Ali for the valuable suggestions, encouragements, support and guidance. I feel honored and humbled to have such esteemed members in my committee.

I would like to extend my thanks to the Hall A DVCS Collaboration, for all the discussions, encouragements, ideas and advises. My special thanks to Dr. Carlos Muños-Camacho, for guiding us through every step, sharing his expertise in data analysis and helping us out with technical and software setup, Dr. Julie Roche for all the inspiring questions, instilling confidence through her warmth and encouragements, Dr. Alexandre Camsonne for sponsoring me at JLab and his assistance with the experimental shifts, many times during the middle of the night when things broke and Dr. Kijun Park for the discussions during assembling and re-assembling the DVCS calorimeter. I'm in debt to the wonderful set of DVCS students and my friends, especially Mongi Dlamini, Bushnu Karki, Alexa Stefanko and Frederic Georges for all the warm conversations and sharing of experiences. This work would not be a success without their work during the calibration and analysis process.

I would also like to thank Thomas Hartlove, of ODU for his help and the quality times spent working on various projects and the Jefferson Lab - Hall A Collaboration, specially Dr. Bogdan Wojtsekhowski, Ole Hansen, Albert Shahinyan and the members of the GMp experiment for their support, help and insights.

My sincere gratitude to Dr. Gail Dodge, who was the chair of the department when I joined, for her help and advises and providing me with the best possible situation to carryout my academic research, Dr. Charles Sukenik for continuing and furthering the 
legacy, Dr. Lepsha Vuskovic and Dr. Alexander Godunov for their services, support and help as Graduate Program Directors. I would like to take a moment to remember Dr. Mark Havey, then Graduate Admissions Director and my first contact at ODU. He had such a warm personality and will be truly missed.

I would also like to thank the extra ordinary experimental nuclear physics community at ODU for the valuable seminars and providing an exiting, vibrant and friendly working environment. My special gratitude to Dr. Larry Weinstein and Dr. Robert Bennett for initiating me in the field of experimental nuclear physics.

I would like to thank the unsung heroes of an international graduate student's life at ODU and JLab. The physics department staffs Lisa Okun, Annette Guzman-Smith and Delicia Malin, the staff at the ODU VISA office, Charlette Kimbro and her extraordinary ITS team and the people at JLab for all their help, support and assistance with all the paper work and everything else that made life easier.

A special thank you goes to Justin Mason for all his work supporting the TAs at ODU physics department. I'm always Indebted to the support and understanding extended by Dr. Perry Neram with the GTA responsibilities specially during the final days of my thesis writing and defense.

I would like to thank all my teachers from grade school to graduate school. My special thanks to Mr. N Suresh, my advanced level physics teacher for instilling an interest of physics in me, All the wonderful faculty members of the physics departments at University of Peradeniya and ODU, and Dr. Josef Dudek for extending his help and concerns when needed.

I had the fortune to meet some extra ordinary people in my life at UoP and Hampton Roads who became great friends. I would like to thank Avanthi Mawathage, Safra Niyaz, Shafraz Omer, Manoj Chandrabose and my fellow students at UoP and ODU for all their support, and for being such wonderful people. My heartfelt thanks to all my Sri Lankan friends and family in Hampton Roads for the warmth with which they welcomed me here, gave me a home away from home and for the many camping trips and volleyball games. I will always cherish the many many end of workday coffee chats at Dunkin with Nanda Karthik, Senthil Raja, Viraj Premathilake, Randika Gamage and Anton Fernando.

I take this opportunity to express my deepest gratitude to my parents, my sister, brotherin-law and their two kids and My lovely wife and my extended family for the love, support and understanding throughout the years.

Finally, and most importantly all praise and thanks belong to the almighty. 


\section{TABLE OF CONTENTS}

LIST OF TABLES. viii

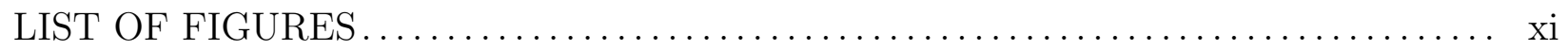

Chapter

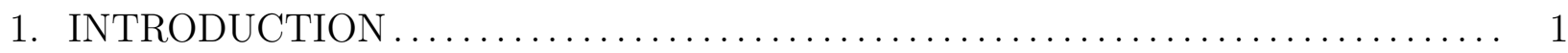
1.1 UNDERSTANDING THE INTERNAL MAKEUP AND STRUCTURE OF

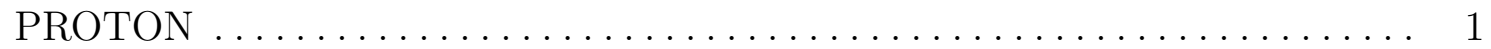

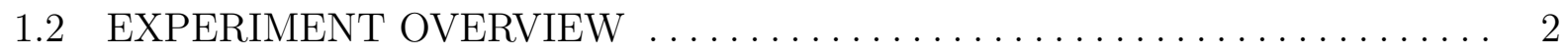

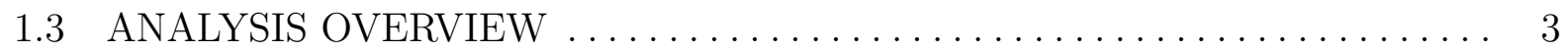

1.4 CROSS-SECTION EXTRACTION METHOD $\ldots \ldots \ldots \ldots \ldots \ldots \ldots \ldots \ldots .4$

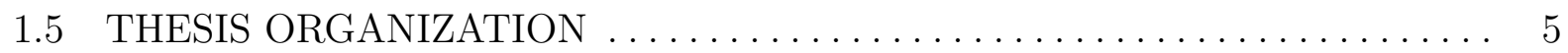

2. PROBING A PROTON THROUGH ELECTRON SCATTERING EXPERIMENTS 6

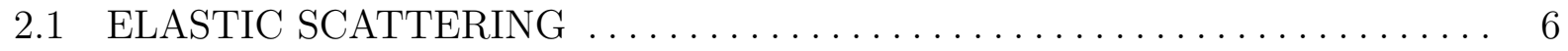

$2.2 \quad$ DEEP INELASTIC SCATTERING $\ldots \ldots \ldots \ldots \ldots \ldots \ldots \ldots \ldots \ldots \ldots$

2.3 GENERALIZED PARTON DISTRIBUTIONS $\ldots \ldots \ldots \ldots \ldots \ldots \ldots \ldots \ldots \ldots$.

2.4 DEEPLY VIRTUAL COMPTON SCATTERING - ACCESSING GPDS IN

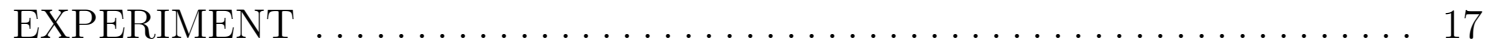

2.5 EXPERIMENTAL OVERVIEW OF DVCS AROUND THE WORLD . . . . . 20

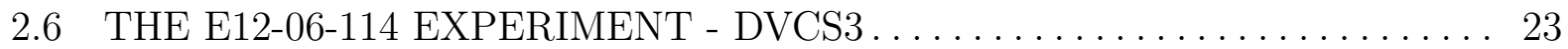

3. EXPERIMENT SETUP AND INSTRUMENTATION $\ldots \ldots \ldots \ldots \ldots \ldots \ldots \ldots \ldots \ldots$

3.1 THOMAS JEFFERSON NATIONAL ACCELERATOR FACILITY] . . . . . . 26

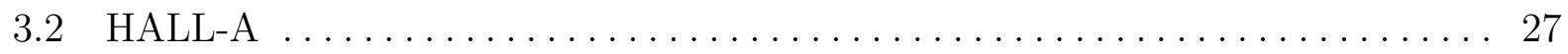

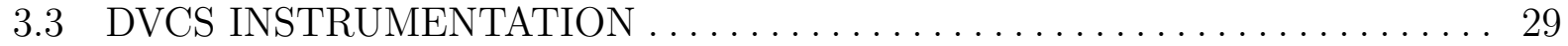

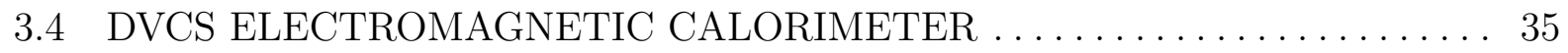

$3.5 \quad$ DATA ACQUISITION $(\mathrm{DAQ}) \ldots \ldots \ldots \ldots \ldots \ldots \ldots \ldots \ldots \ldots \ldots \ldots \ldots$

4. HRS DETECTOR CALIBRATION AND EFFICIENCY ANALYSIS. . ........... 39

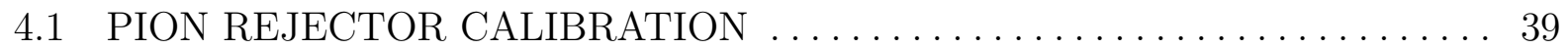

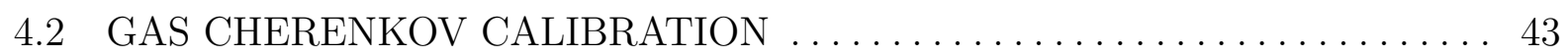

4.3 TARGET VERTEX RECONSTRUCTION AND OPTICS STUDIES $\ldots . . . .48$

4.4 TRACKING STUDIES AND TRACKING EFFICIENCIES $\ldots \ldots \ldots \ldots \ldots \ldots$.

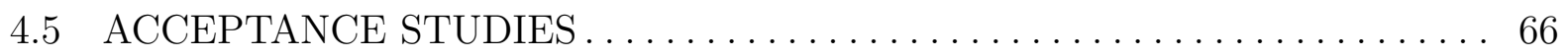

4.6 TRIGGER EFFICIENCY STUDIES $\ldots \ldots \ldots \ldots \ldots \ldots \ldots \ldots \ldots \ldots \ldots \ldots$ 
5. ELECTRON SELECTION, SPECTROMETER NORMALIZATION AND TRIG-

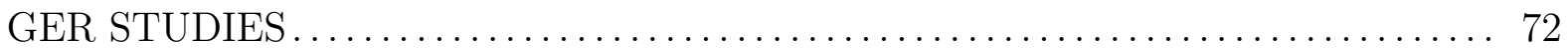

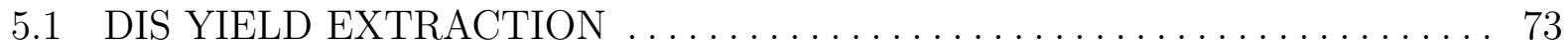

5.2 DIS TRIGGERS AND PRE-SCALE AMBIGUITY $\ldots \ldots \ldots \ldots \ldots \ldots \ldots$

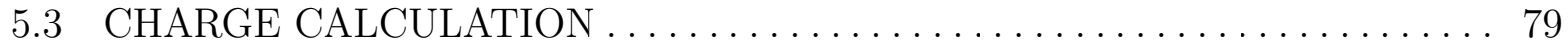

5.4 EXPERIMENTAL EFFICIENCY CORRECTIONS $\ldots \ldots \ldots \ldots \ldots \ldots \ldots$

5.5 PHASE-SPACE AND RADIATIVE CORRECTION $\ldots \ldots \ldots \ldots \ldots \ldots \ldots$

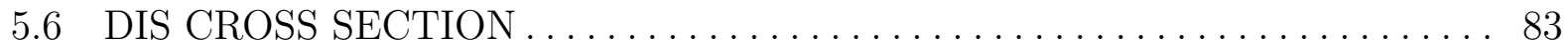

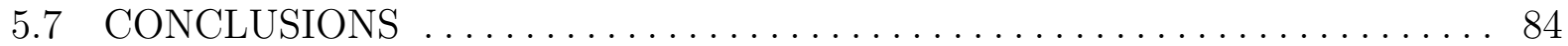

6. DVCS CALORIMETER AND PHOTON SELECTION $\ldots \ldots \ldots \ldots \ldots$

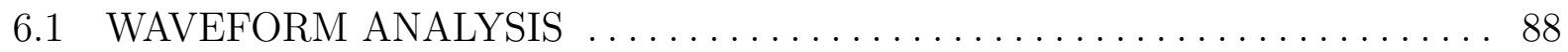

6.2 CALORIMETER ENERGY CALIBRATION $\ldots \ldots \ldots \ldots \ldots \ldots \ldots \ldots$

6.3 HRS-CALORIMETER COINCIDENCE TIME CORRECTION $\ldots \ldots \ldots \ldots 92$

6.4 CLUSTERING ALGORITHM $\ldots \ldots \ldots \ldots \ldots \ldots \ldots \ldots \ldots \ldots \ldots \ldots \ldots \ldots \ldots \ldots \ldots$

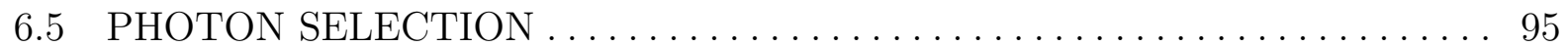

6.6 CONTAMINATION SUBTRACTION $\ldots \ldots \ldots \ldots \ldots \ldots \ldots \ldots \ldots \ldots \ldots \ldots \ldots$

6.7 MONTE-CARLO EVENT GENERATION AND GEANT4 SIMULATION . . 99

6.8 CALIBRATION AND SMEARING OF THE SIMULATION $\ldots \ldots \ldots \ldots . .99$

6.9 CALORIMETER MULTI CLUSTER, MISSING MASS AND EXCLUSIVITY 100

7. DVCS CROSS SECTION ANALYSIS]. . . . . . . . . . . . . . . . . . . . . . . . . . . 106

7.1 EXPERIMENTAL EFFICIENCY CORRECTIONS . . . . . . . . . . . . . . 106

7.2 CROSS SECTION EXTRACTION METHOD $\ldots . . \ldots \ldots \ldots \ldots \ldots \ldots$

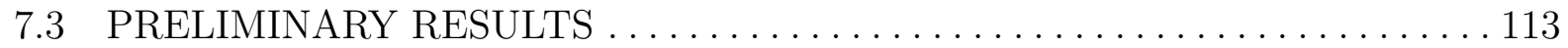

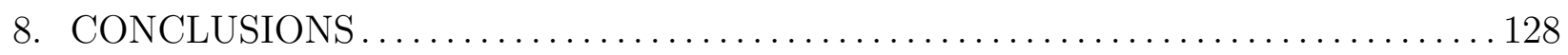

APPENDICES

A. PION REJECTOR RENORMALIZATION PROCEDURE AND STABILITY

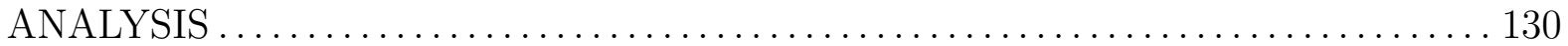

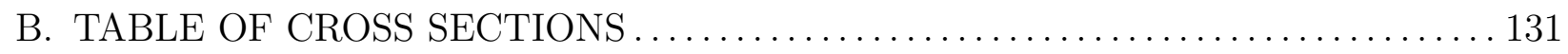

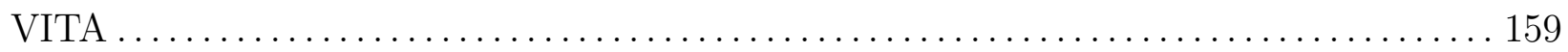




\section{LIST OF TABLES}

Table

1 DVCS3 runs were taken in 9 different kinematic settings, in 3 different run periods spanning 2 years. The experiment used multiple beam energies up to $11 \mathrm{GeV}$. The total acquired charge per kinematic listed here are adjusted to the DAQ

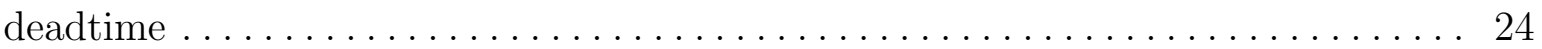

2 This table summarizes the renormalization weighting factors W1 and W2 of the two layers of the Pion Rejector for each kinematic setting as the average over all the runs in the kinematic together with the standard deviations. . . . . . . . . 43

3 This table shows the extracted calibration parameters for the $4^{\text {th }}$ phototube of the Gas Cherenkov. This parameters are then used to calibrate the Gas Cherenkov and extract the photo electron yield per electron travelled through

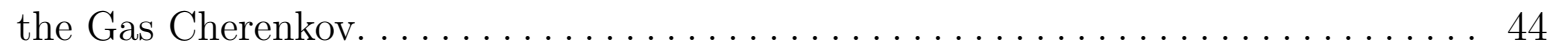

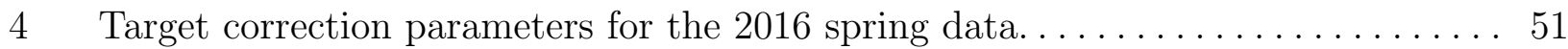

5 Target offset values for each kinematic setting. $Z_{\text {Offset }}-$ Optics is used in the

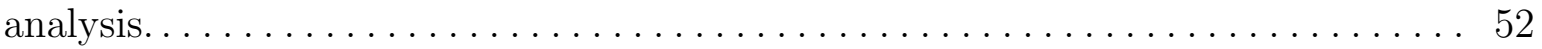

$6 \quad$ Accepted Event Fraction values for different classes of events for Run 14228 of Kin $36 \_2$ shows the effect of varying tracking efficiency with different classes of

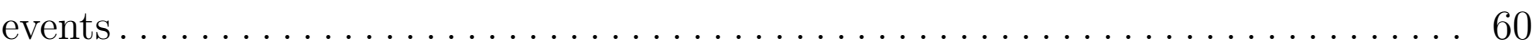

7 Correction Factors computed for Two Track events and and all Multi Track candidates events using election selections with total Pion Rejector sum and Pion Rejector Cluster sum. Only higher number of track candidate events shows a large uncertainty in our extracted correction factors, provinding evidence to the idea these events could contain additional low energy electron track . . . . . . . 65

$8 \quad$ This table summarizes the results for tracking efficiency corrections expressed as a multiplicative correction factor for each kinematics. $\ldots \ldots \ldots \ldots \ldots \ldots \ldots$

$9 \quad$ This table summarizes the RCut values as defined by A. Stefanko [75] . . . . . . 67

10 This table summarizes the trigger efficiency for Fall $2014 \ldots \ldots \ldots \ldots \ldots \ldots \ldots$. . . 70

11 This table summarizes the trigger efficiency for Spring 2016. Spring 2016 has 17 multi trigger runs. S2M detector was always in the trigger in these runs and hence an unbiased trigger efficiency for S2M was not estimated. Instead the efficiency between 2016 Spring and Fall runs were assumed to be similar and the value of

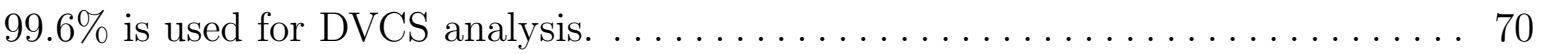


12 This table summarizes the trigger efficiency for Fall 2016. Fall 2016 has 9 dedi-

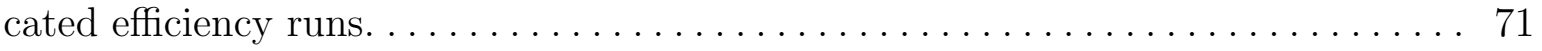

13 This table summarizes the BCM calibration Coefficient from B. Karki [50] ...... 80

14 This table shows experimental correction factors. $\ldots \ldots \ldots \ldots \ldots \ldots \ldots \ldots$

15 This table shows the Phase-Space and Radiative Correction factors from A. Stefanko [74], and B. Karki [53]. Subscript or Superscript B and E denotes the computations from Bishnu Karki with two different simulations. A- denotes the computation by Alexa Stefanko with a $3_{r d}$ simulation. All three computations are using the same DIS parametrization from [21] $\ldots \ldots \ldots \ldots \ldots \ldots \ldots$

16 This table shows the extracted DIS Cross-Section from a previous study by A. Stefanko $\left(A_{\text {old }}\right)$ and B. Karki $\left(B_{\text {old }}\right.$ and $\left.E_{\text {old }}\right)$ with a New study I did using the same DIS Cross section normalizations but a standardized yield $\begin{array}{lllllllllll}\text { analysis. } & \text { All cross sections are expressed as a fraction } \frac{\sigma_{i}}{\sigma_{s}} \text { where } \sigma_{i} \in\end{array}$ $(\mathrm{A}, \mathrm{B}, \mathrm{E})$ and $\sigma_{s}$ is the reference cross-section $\ldots \ldots \ldots \ldots \ldots \ldots \ldots \ldots \ldots$

17 This table summarizes the photon energy cut, Calorimeter cluster threshold, $\pi^{0}$ invariant mass $M_{\pi^{0}}$ and $\sigma_{M_{\pi^{0}}}$ for each run period of each kinematics. . . . . . . 97

18 This table shows the missing mass cut region for each kinematic setting. . . . . . . . 102

19 This table summarizes the average live time correction factors and live time adjusted total accumulated charge. We compute the charge and LTCF run by run and sum them together to get the total accumulated charge $\left(\right.$ Adjusted $\left.^{\text {Total }}\right)$. Since the live time correction is already included in the listed charge, we do not apply a correction for it again. Charge calculation is described in section 5.3 and the deadtime corrections are discussed in section $5.4 .1 \mid \eta_{\text {Polarization }}$ is described

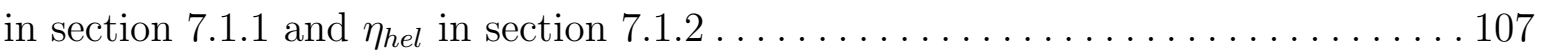

$20 \quad$ Unpolarized cross section for Kin 361 in $\mathrm{pb} / \mathrm{GeV}^{4}$

$21 \quad$ Helicity-Dependent cross section for Kin 361 in $\mathrm{pb} / \mathrm{GeV}^{4} \ldots \ldots \ldots \ldots \ldots$

$22 \quad$ Unpolarized cross section for Kin 362 in $\mathrm{pb} / \mathrm{GeV}^{4} \ldots \ldots \ldots \ldots \ldots \ldots \ldots \ldots$

$23 \quad$ Helicity-Dependent cross section for Kin 362 in $\mathrm{pb} / \mathrm{GeV}^{4} \ldots \ldots \ldots \ldots \ldots \ldots$

24 Unpolarized cross section for Kin 363 in $\mathrm{pb} / \mathrm{GeV}^{4} \ldots \ldots \ldots \ldots \ldots \ldots \ldots$

$25 \quad$ Helicity-Dependent cross section for Kin 363 in $\mathrm{pb} / \mathrm{GeV}^{4} \ldots \ldots \ldots \ldots \ldots \ldots$

$26 \quad$ Unpolarized cross section for Kin 481 in $\mathrm{pb} / \mathrm{GeV}^{4} \ldots \ldots \ldots \ldots \ldots \ldots \ldots$

27 Helicity-Dependent cross section for Kin 481 in $\mathrm{pb} / \mathrm{GeV}^{4} \ldots \ldots \ldots \ldots \ldots \ldots$ 
28 Unpolarized cross section for Kin 482 in $\mathrm{pb} / \mathrm{GeV}^{4} \ldots \ldots \ldots \ldots \ldots \ldots \ldots$

$29 \quad$ Helicity-Dependent cross section for Kin 482 in $\mathrm{pb} / \mathrm{GeV}^{4} \ldots \ldots \ldots \ldots \ldots \ldots 1$

$30 \quad$ Unpolarized cross section for Kin 483 in $\mathrm{pb} / \mathrm{GeV}^{4} \ldots \ldots \ldots \ldots \ldots \ldots \ldots$

$31 \quad$ Helicity-Dependent cross section for Kin 483 in $\mathrm{pb} / \mathrm{GeV}^{4} \ldots \ldots \ldots \ldots \ldots$

32 Unpolarized cross section for Kin 484 in $\mathrm{pb} / \mathrm{GeV}^{4} \ldots \ldots \ldots \ldots \ldots \ldots \ldots$

33 Helicity-Dependent cross section for Kin 484 in $\mathrm{pb} / \mathrm{GeV}^{4} \ldots \ldots \ldots \ldots \ldots$

34 Unpolarized cross section for Kin 601 in $\mathrm{pb} / \mathrm{GeV}^{4} \ldots \ldots \ldots \ldots \ldots \ldots \ldots \ldots$

$35 \quad$ Helicity-Dependent cross section for Kin 601 in $\mathrm{pb} / \mathrm{GeV}^{4} \ldots \ldots \ldots \ldots \ldots \ldots \ldots$

$36 \quad$ Unpolarized cross section for Kin 603 in $\mathrm{pb} / \mathrm{GeV}^{4} \ldots \ldots \ldots \ldots \ldots \ldots \ldots$

$37 \quad$ Helicity-Dependent cross section for Kin 603 in $\mathrm{pb} / \mathrm{GeV}^{4} \ldots \ldots \ldots \ldots \ldots$ 


\section{LIST OF FIGURES}

Figure

$1 \quad$ Figure shows a Feynman diagram of an elastic $e p \rightarrow e^{\prime} p^{\prime}$ scattering in the single photon exchange approximation. Incoming electron emits a virtual photon in the presence of a proton and scatters off. The virtual photon is absorbed by the proton and the proton momentum is changed as a result. $k, k^{\prime}, P$ and $P^{\prime}$ are the four-vectors of incoming electron, scattered electron, initial proton and the final

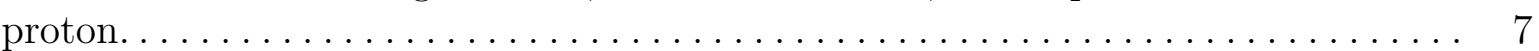

2 Figure 2 2a gives the measured elastic cross section as a function of scattering angle from [61]. The measured cross section deviated from the prediction for a point-like particle (equation 3), point charge(5) and point charge with anomalous magnetic moment (4) indicating a diffused structure for the proton. Figure $2 \mathrm{~b}$ gives the ratio of measured DIS cross section to the Mott cross section with $Q^{2}$ for different values of the invariant mass $W$ from [54].The dotted curve indicates the prediction for the ratio as inferred from previous experiments with low beam energy. The open $(W=3 \mathrm{GeV})$ and closed $(W=2 \mathrm{GeV})$ circle points following a straight-line indicating that the electrons are in fact scattering off of a point-like particle within the proton. These particles are called Partons at the time and later identified with the quarks suggested by Gell-Mann[36] and Zweig[80] . . . . 8

3 Figure $3 \mathrm{a}$ shows a Feynman diagram of an inelastic $e p \rightarrow e^{\prime} X$ scattering in the single photon exchange approximation. Incoming electron emits a virtual photon in the presence of a proton and scatters off. The virtual photon interacts with the proton and breaks it apart. $k, k^{\prime}$, and $P$ are the four-vectors of incoming electron, scattered electron and initial proton and $X$ is the sum of all possible hadron final states. Figure $3 \mathrm{~b}$ | shows the Feynman diagram of DIS in the Bjorken limit where the virtual photon scatters of of a parton inside the proton carrying a fractional momentum $x$ of the proton. $\ldots \ldots \ldots \ldots \ldots \ldots \ldots \ldots \ldots \ldots \ldots \ldots \ldots \ldots \ldots \ldots \ldots \ldots \ldots \ldots \ldots$

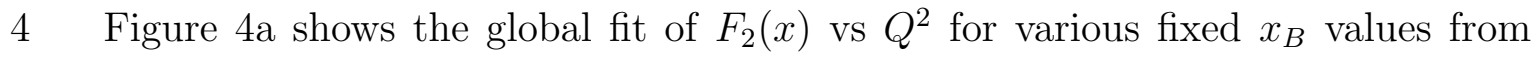
[55]. Scaling is observed for $0.032 \leq X_{B} \leq 0.25$ region. For smaller $x_{B}$ values $F_{2}$ increases with $Q^{2}$ and for larger $x_{B}$ values $F_{2}$ decreases with increasing $x_{B}$ violating the scaling. This variation is explained by DGLAP evolution [7] discussed in section 2.2 .2 . Figure $4 \mathrm{~b}$ shows the global fit for the valence distribution with $x$ for $Q^{2}=10 \mathrm{GeV}$. The plot shows the existence of twice as large up quark distribution than down quark distribution in the valence region. We also see that the valence quark distribution becomes much smaller in the small $x$ region while the gluon and the sea quark distribution rises as if the proton is made up of a soup of gluons, quarks and anti-quarks of all flavors. $\ldots \ldots \ldots \ldots \ldots$ 
$5 \quad$ Figure shows the leading order - twist 2 handbag diagram of underlying virtual compton scattering of the $e p \rightarrow e^{\prime} p^{\prime} \gamma$ process. Virtual photon emitted by the incoming electron interacts with a parton picked out from a nucleon in a billiardball like collision. The parton momentarily absorbs this virtual photon and then emits a real photon when it returns back to the nucleon, keeping the nucleon intact. $x+\xi$ is the longitudinal momentum fraction carried by the struck quark and $x-\xi$ is the longitudinal momentum fraction carried by quark after emitting the real photon when returning to the final state. $t=\Delta^{2}=\left(p^{\prime}-p\right)^{2}$ is the total transfer of 4-momentum squared at the proton vertex. $\xi \sim \frac{x_{B}}{2-X_{B}}$ is called the

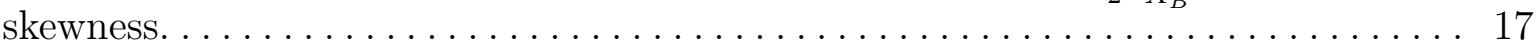

6 Figure shows the DVCS process and Bethe-Heitler. In DVCS the final state photon is emitted from the nucleon but in $\mathrm{BH}$ the final state photons are bremsstrahlung radiation from either the incoming or the scattered electron. . ... 18

7 Figure shows the handbag diagram of Deeply Virtual Meson Production. The process has an additional layer of theoretical complexity via the pion distribution amplitude, DA. The soft part has additional GPDs called transversity GPDs. . . . 19

$8 \quad$ Figure shows the lepton scattering plane and hadron scattering plane from [10]. $\phi$ defined in eqaution 40 , is the azimuthal angle used for harmonic expansion in

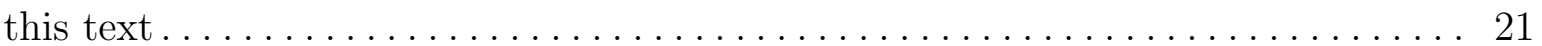

$9 \quad$ Figure shows the combined kinematic coverage for GPD studies via DVCS pastpresent-and future [2]. JLab kinematics are mostly in the valence quark region. After HERA, the proposed Electron Ion Collider will provide coverage in to the

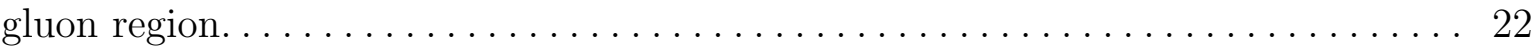

$10 \quad$ Figure shows the combined kinematic coverage for DVCS3 experiment from real acquired data. each scatters are roughly 2000 points. Blue is $x_{B}=0.6$, Green is

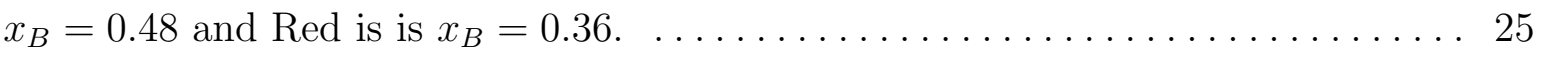

11 A cartoon diagram of the DVCS3 experiment setup in Jefferson Lab, Hall A. Scattered electrons are detected in the Left High Resolution Spectrometer and

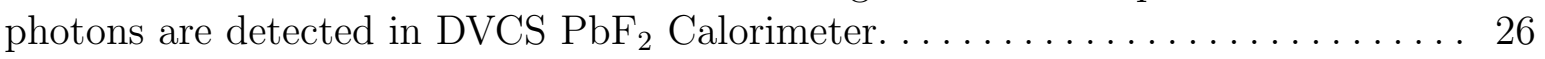

12 A schematic diagram of the Continuous Electron Beam Accelerator Facility (CEBAF) of Jefferson Lab. The diagram shows the two linacs and the positions of the 4 experimental halls. Electrons are accelerated up to $45 \mathrm{MeV}$ in the injector,

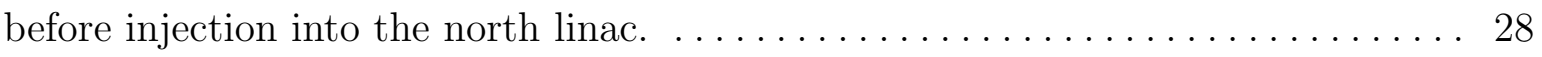

13 Diagram of the experimental Hall A setup. Target chamber is in the center. . . . . 29

14 Cartoon diagrams of the LHRS detector stack showing the various particle and

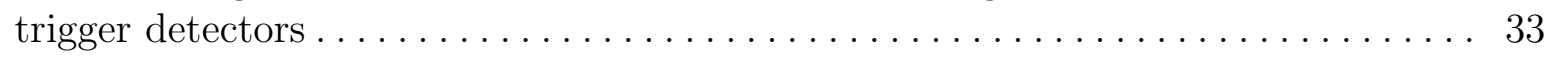


15 Hall A LHRS Pion Rejector has 2 identical layers of 34 Pb-Glass blocks. . . . . . . . 34

16 Gas Cherenkov in Hall A DVCS is a threshold detector with $1.2 \mathrm{~m}$ long chamber filled with atmospheric pressure $\mathrm{CO}_{2}$ that could distinguish between electron and

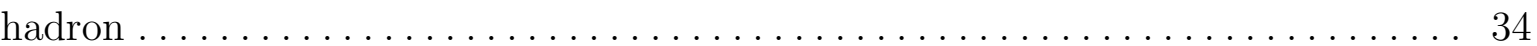

17 Side and top view of the Hall A vertical Drift Chamber. VDC has 2 chambers (Top and Bottom) at 335mm vertical distance between each other with 2 perpendicular wire planes (U and V) each having 368 sense wires per plane with $4.24 \mathrm{~mm}$ wire spacing. It sits at a 45 degree angle to the nominal particle trajectory with bottom chamber at $Z_{\text {focal plane }}=0$ and uses standard Argon $/$ Ethane $(62 \%-38 \%$

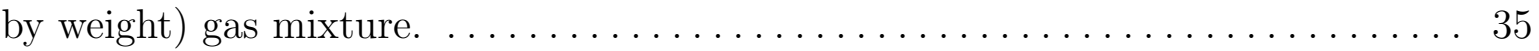

18 Figure shows the assembled DVCS calorimeter before installing the front and back panels and the black box. $\ldots \ldots \ldots \ldots \ldots \ldots \ldots \ldots \ldots \ldots \ldots \ldots \ldots \ldots \ldots \ldots \ldots \ldots \ldots$

19 Figure shows PRL1 sum (Blue) and PRL2 sum (Red) for 4 different runs with 3 different kinematics showing the gain mis-alignment of minimum ionizing peak between layers (around channels 100-200). It also demonstrates that the gain is a function of the kinematic setting as evidence by the different mis-alignment among different kinematic runs and similar mis-alignment between the same kinematics

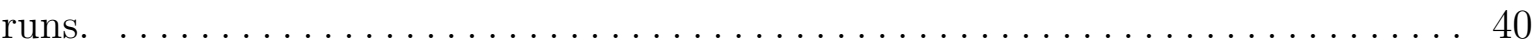

20 Amplitude spread of PRL1 Vs PRL2. Red oval is to illustrate the bulk of the single track electron events. these events can be selected and re-normalized using the weighting factors W1 and W2 so that they lie on a 45 degree line and the electron peak of the re-normalized Pion Rejector sum is at ADC Chan 1000. . . . 41

21 Figure shows the renormalization for run 12985. Plots on the Left shows 2D and 1D Pion Rejector Spectrum before renormalization and the plots on the right shows the same after renormalization. 1D PRL1 spectrum is given in blue and PRL2 in red. We can see that the renormalization procedure automatically aligns the minimum ionizing peaks of the two layers. $\ldots \ldots \ldots \ldots \ldots \ldots \ldots \ldots \ldots \ldots$

22 Each individual photo tube spectrum of the Gas Cherenkov in LHRS were fitted using a two part gaussian and a second order polynomial tail to extract the position and width of pedestal and single photo electron peak. Figure $22 \mathrm{a}$ a shows the fit for the $4^{\text {th }}$ photo tube. Figure $|22 \mathrm{~b}|$ shows Cherenkov amplitude spectrum with two different calibration. Blue spectrum shows the initial calibration performed by the GMp group. Red spectrum shows the calibration performed for verification. The test calibration recovered a cleaner Poisson distribution. . . . . . . 44 
23 Poisson fit on central 4 PMT's of the Gas Cherenkov for DVCS efficiency run 10419 on carbon target taken in fall 2014, with scintillator triggers. Here, only the electron events from Pion Rejector with $90 \%$ of the Cherenkov light collected by the PMT in question are selected. Since this selection considers only one out of the 10 phototubes, it also rejects $90 \%$ of the Multi-Electron events seen as a long non-poisson tail in Figure $22 \mathrm{~b}$. New photo electron yield is roughly about

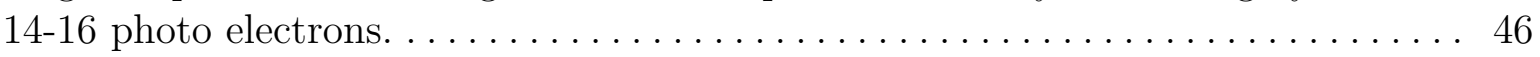

24 Normalized Pion Rejector sum with various Cherenkov cuts (left) and Cherenkov spectrum with various Pion Rejector Cuts (right) are shown here. Events that are on the right to the red vertical line on both plots, that is, events with more than 1.5 photo electron in the Cherenkov and NormalizedPRSum greater than $60 \%$ of the full electron peak with more than $20 \%$ of the full electron peak in PRL1 are considered "Good Electron". 1. PE is chan 100 in Cherenkov spectrum. Left plot shows very minimal effect if increasing the cerenkov cut up to $7 \mathrm{PE}$ due to the already high efficiency of the 1.5 PE cut. Right plot shows that the Pion Rejector cut effectively eliminating the multi-electron bacground - the non-Poisson tail. . . 47

25 Left: Medium energy electron pile-up events as selected by CERElectron $\cap$ PRMediumElectron, reconstructed in the geometrical $y$ vs. $x$ plane of the first layer of pion rejector. This spectrum shows a localization at the extreme large $x$ end of the Lead-Glass blocks (which is the region of maximal reconstructed momentum and physically at the top, closer to the ceiling of the Hall). Right: Same events after a target vertex cut, which shows that the cluster of events at large $x$ are not coming from the target itself, an indication that these could be

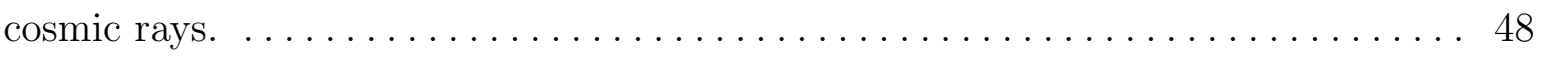

$26 \quad$ Figure shows target vertex distributions for Dummy Target run against $\mathrm{LH}_{2}$ target run, normalized to the run duration. Dummy target (Green) shows the scattering from the $\mathrm{Al}$ end caps leaking into $\mathrm{LH}_{2}$ spectrum (Blue). Cutting $1 \mathrm{~cm}$

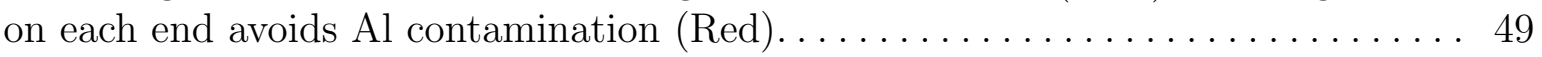

27 Target vertex distribution, normalized to the the event count plotted for 3 different kinematic settings, Kin 361 (Blue), 362 (Red), 363 (Green) shows a shift in the target center, kinematic to kinematic. $\ldots \ldots \ldots \ldots \ldots \ldots \ldots \ldots \ldots \ldots \ldots \ldots \ldots \ldots \ldots \ldots$

28 Target vertex cut for all kinematic settings. Blue is the reconstructed target, Green is the Target Cut and Red is the selected events after applying all electron

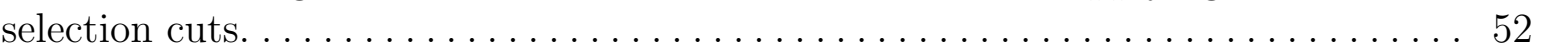

29 Each nominal track activates around 4-6 wires in a single wire plane (clusters). $\theta_{Q i}$ is local drift angle and $Q i$ is local cross over position $\ldots \ldots \ldots \ldots \ldots \ldots 4$ 
30 Track Co-Ordinates reconstructed at Q3 Magnet for Run 14228 of Kin 36_2. Plot on top left shows the $1^{\text {st }}$ reconstructed track for all the events that have more than one reconstructed track. Top right shows the track co-ordinates for only events with one reconstructed track. Bottom plots shows the same plot for identified electron tracks only. Electron tracks are selected based on gas Cherenkov and Pion Rejector. The localization around $Y=0$ and $X= \pm 0.3$ together with the crescent shape suggest showering from the edges of the Q3 magnet $\ldots \ldots \ldots \ldots 57$

31 Track Co-Ordinates reconstructed at Q3 Magnet for Run 14228 of Kin 36_2 for different classes of single track events with good electron. Plot on top left shows the track co-ordinates for all events with only one reconstructed track. Top right shows the events with all single clusters in every wire plane of the VDC. Bottom left plot shows the event with one multiple cluster in one of the wire plane and single clusters in all other three wire planes. Bottom right shows the events with purely single cluster on both wire-planes of one chamber and 2 clusters each on both wire planes of the second chamber. Electron tracks are selected based on gas Cherenkov and Pion Rejector. Even though there are no clear localization here as in Figure 30 its shows evidence that the tracking efficiency reduces with increasing number of clusters in wire plane $\ldots \ldots \ldots \ldots \ldots \ldots \ldots \ldots$

32 Track Co-Ordinates reconstructed at Pion Rejector Layer 1 for Run 14228 of Kin 36_2 for different classes of events with good electron (passing the PID cuts). These are events depositing at least $60 \%$ of full electron peak energy in the Pion Rejector and hence expected to be within its boundary. Plot on top left shows the track co-ordinates for all events with only one reconstructed track. Top right shows the events with at most one multi clusters in any of the wire plane of the VDC. Bottom left shows the events with purely single cluster on both wire-planes of one chamber and 2 clusters each on both wire planes of the second chamber. Bottom left plot shows the events with more than one reconstructed tracks. The accepted event percentage from equation 55 for each event class is shown on the plot. acceptance drops to nearly half for 2M2S events from the other two types

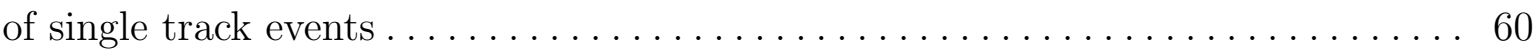

33 Pion Rejector sum for single track (blue), 2 track candidate(red), 3 track candidate (green), 4-track candidate (black) and 5 track candidate (purple) events in log scale. Only 2 track events are showing a considerable electron peak. $\ldots . \ldots 62$

34 Left plot shows the raw distribution of a sample event with two clusters in the pion rejector. Right plot shows an identified two cluster event after the $2^{\text {nd }}$ iteration of the cluster finding algorithm. $\ldots \ldots \ldots \ldots \ldots \ldots \ldots \ldots \ldots \ldots \ldots \ldots \ldots \ldots \ldots \ldots \ldots$

35 Plot shows Normalized Pion Rejector sum spectrum of raw sum in blue and

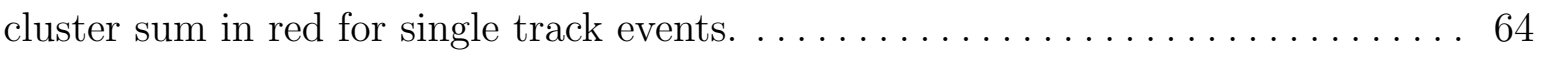


36 Figure shows the application of RFunction cut for kin 603 on each plane the functions are defined. Left is the raw distribution and right is after the cut. . . . . 67

37 Typical timing spectra of different triggers. Blue spectrum shows all events and Red spectrum shows Good Single track electron events, with the green lines indicate the cut region for determining good timing signal. Fig 1-3 are from High resolution DVCS TDC and figure 4 is from LHRS TDC . . . . . . . . . . . 69

38 On the left, $\frac{\delta P}{P}$ spectrum for Kin 481 run 12518 without $S 0 \cap C E R$ in the trigger. On the right $\delta p$ spectrum for same kinematic run 12541 with $S 0 \cap C E R$ in the trigger. Shape of the spectrum suggests that there are events missing in the low

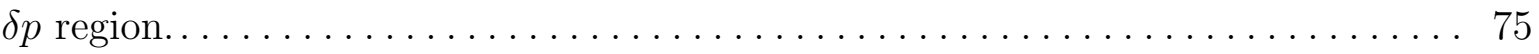

39 Plots show overlaid S0-ARSStop and S2-ARSStop timing spectra (time on the vertical axis) as functions of the reconstructed tract $\delta p / p$ value on the horizontal axis, for two of the spring 2016 runs. The spread following the red line shows the S0 timing and green line is S2 timing. S0 spectrum shows about a 50 ns spread. Plot on the left is for Run 12518 without $S 0 \cap C E R$ in the trigger. Plot on the right for run number 13100 shows an additional set of events with an unexpected S0 timing in the presence of $S 0 \cap C E R$ trigger in low x region. The numbers in parentheses next to the run number in the plot titles are the pre-scale factor for DIS, $S 0 \cap C E R$ and $S 0 \cap S 2$ triggers in that order $\ldots \ldots \ldots \ldots \ldots \ldots \ldots$

40 Plot shows $S 2 \cap C E R$ coincidence time with respect to ARSStop separated by triggerPatterWord tags. Blue spectrum is Inclusive DVCS, Red is Inclusive DIS and Green in Exclusive $S 0 \cap C E R$. Events from $S 0 \cap C E R$ coincidence trigger shows $S 2 \cap C E R$ coincidence time peaks, even though they were not tagged. (Here, Exclusive means events with only the spoken trigger tag and Inclusive means events with spoken trigger tag with other additional trigger tags $) \ldots \ldots 76$

41 Plot shows yield corrected $\delta p$ spectrum for Run 13110 using equation 67 . Blue spectrum is scaled DIS (uncorrected). Green is the corrected spectrum. Magenta is GoodElectron $\cap$ Calo, Yellow is scaled DIS $\cap$ !Calo the Red is scaled Exclusive S0CERก!Calo. Green spectrum is the sum of Red, Yellow and Magenta and

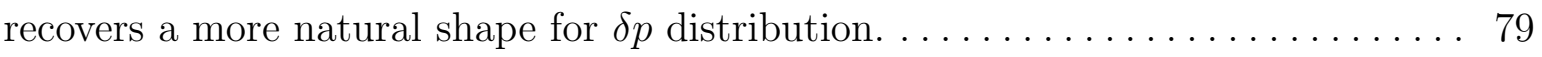

42 Plot shows the run-by-run extraction of DIS cross section for Kin 603 using equation 75. Red (Bishnu) Blue(Alexa) and Green (Eric) are using 3 different $\alpha$, Гand $\eta_{R}$ given in Table 15 , which were computed with the same cross-section parametrization but with 3 different simulations. All extraction of the cross section uses extracted Yield with the same acceptance cuts, implying the variations among them are due to the uncertainties of normalizing the model cross section rather than the uncertainty in the acceptance. The numbers stated are the average percentage variations of each extraction from the reference cross section for

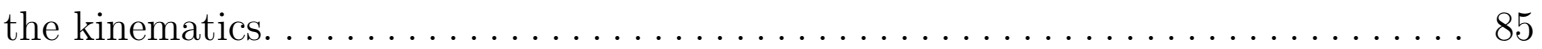


43 Figure shows the run-by run DIS cross section for Kin 483, a spring 2016 kinematics. Figure (a) shows the cross section using the old yield extraction and Figure (b) shows the cross section using the new yield extraction, indicating the improvement made by the Equation 67 in Yield extraction. Red (Bishnu) and Blue(Alexa) are using 2 different $\alpha$, $\Gamma$ and $\eta_{R}$ given in Table 15, which were computed with the same cross-section parametrization but with 2 different simulations. All extraction of the cross section uses extracted Yield with the same acceptance cuts. The simulation labelled Eric was not available for this kinematics. The numbers stated are the average percentage variations of each extraction from the reference cross section for the kinematics, negative sign implying the

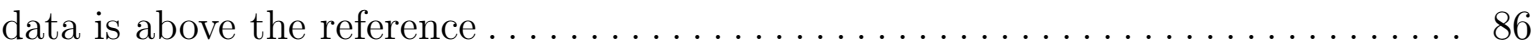

44 Figure shows the one pulse and two pulse fit sample events. The signal pulse is is in blue and the fit using the reference shape is in red. This results are courtesy

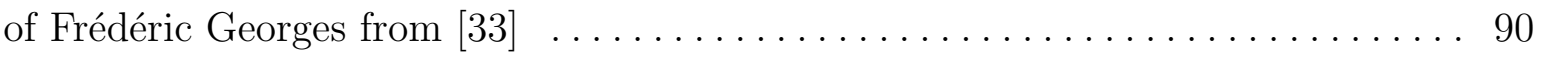

45 Figure shows the reconstructed $\pi^{0}$ invariant mass before (blue) and after (red) the $\pi^{0}$ calibration for a fall 2016 data performed by Frédéric Georges [33]. The black reference line indicates the expected $\pi^{0}$ mass. The result demonstrate that the

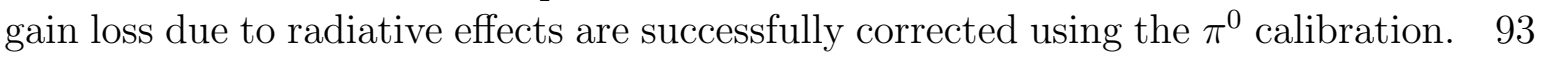

46 Figure shows the width of the timing distribution per calorimeter block after each consecutive timing correction. The black star spectrum indicates the final corrected time distribution. Timing correction for DVCS3 was performed by

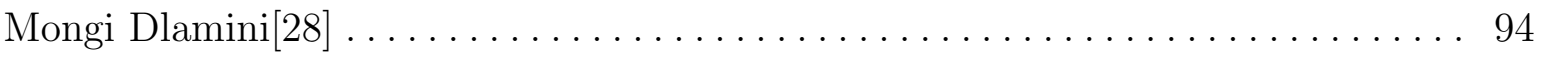

47 Figure shows the coincidence time spectrum of all detected photons. The blue spectrum is obtained by clustering in the whole $[-15,15]$ ns window. The red spectrum is obtained by doing the clustering in each individual timing windows of $[-3,3] \mathrm{ns},[-11,5] \mathrm{ns}$ and $[5,11] \mathrm{ns}$. Red spectrum includes an additional

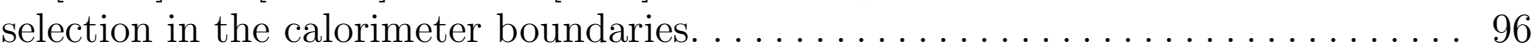

48 Figure (a) shows the full calorimeter and (b) shows the calorimeter fiducial region for photon selection, for Kin 483. (b) is more finely binned than $\mathrm{F}(\mathrm{a}) . \ldots \ldots \ldots 97$

$49 \quad$ Left figure shows the reconstructed missing mass spectrum for kin 481 in Black. This spectrum includes the contamination from accidental coincidences (Magenta) and when only one photon is detected from a $\pi^{0}$ decay pair (Green spectrum is simulated estimate of this case). The blue spectrum is from the estimated DVCS event candidates after the contamination subtraction (Black - Green Magenta). Right figure shows missing mass spectrum from the simulation after smearing (Red) overlaid on top of the missing mass subtracted spectrum of the data (Blue). The black spectrum in the left plot is the simulated missing mass

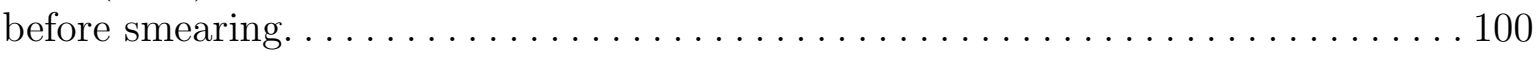


50 Plot shows missing mass spectrum from the simulation after smearing (Red) overlaid on top of the missing mass subtracted spectrum of the data (Blue). Red dotted lines are indicating the missing mass range for photon selection. . . . . . . . 101

51 Left plot shows the extracted cross section at $-0.0118<t^{\prime}<0$, and $\phi_{\gamma \gamma}=172^{0}$ with varied missing mass cut for kin 603 . The blue box extraction are obtained by varying the $M x_{\text {cut }_{\text {min }}}^{2}$ values in $0.2 \mathrm{GeV}$ steps around the central value for the fixed $M x_{c u t_{\max }}^{2}$. The red box extractions are the other way around. Start marked points are the central points. Extracted cross sections are independent of $M x_{c u t}^{2}$ while a strong systematic uncertainty is seen for $M x_{c u t_{m a x}}^{2}$. Right plot shows only the red box extraction of left plot with $M x_{c u t_{\text {max }}}^{2}$ varied in $0.1 \mathrm{GeV}$ steps at $-0.0430<t^{\prime}<0$, and $\phi_{\gamma \gamma}=172^{0}$ for kin 481 . The maximum variation between $\pm 0.06 \mathrm{GeV}$ region around the central cut value is taken to be the systematic

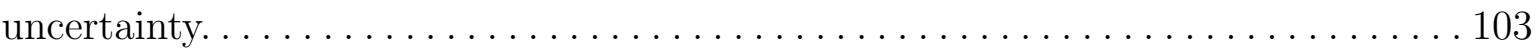

52 Number of clusters recorded in each events are shown in Blue on the left plot. The selected photon Index is shown in Red. The algorithm not always picks the first photon, If we only kept 1 photon in the clustering, either the cases in which the second or higher photon is picked would have been excluded or a photon with a lower likelihood of being from DVCS event would have been picked. Plot on the right shows the photon energy spectrum, Black is for all photon in the true coincidence time window of $[-3,3] n s$, Blue is after the application of Calorimeter fiducial cut discussed in section $\mid 6.5$, Red spectrum is after applying the missing

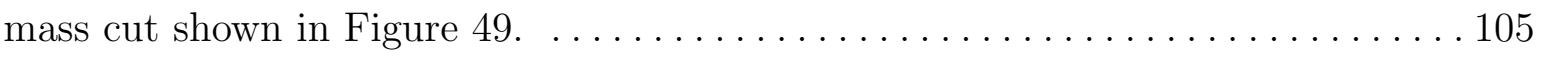

53 The diagrams shows the different internal radiative processes for DVCS . . . . . 108

54 Fitted yield results for Kin 362 unpolarized case is shown here. Reduced $\chi^{2}$ for the overall fit is 1.66 for choice 1 and 1.37 for choice 2 . The points are the experimental yield and the error bars are statistical only. Fit is given as the fill. Parametrization Choices are given in section $\mid 7.2 .4 \ldots \ldots \ldots \ldots \ldots \ldots \ldots \ldots$

55 DVCS Unpolarized cross section for kin 362 at $t_{\text {Bin }}-0.062<t^{\prime}<0.000$ with Beam energy $=8.521 \mathrm{GeV},<Q^{2}>=3.6 \mathrm{GeV}^{2}$ and $\left\langle x_{B}>=0.36\right.$. Closed boxes $/$ Filled colors represents Choice 1 given in section 7.2 .4 . The open boxes/ Polka Squares represents Choice 2 given is section 17.2 .4 . Red curve is the contribution from pure BH cross section. The grey bands around the fits are the statistical uncertainty. Magenta dots represents a parallel analysis by Frédéric Georges [33]. Blue and Brown lines are two global fits given in [57] [56]. The agreement between the different parametrization validates the claim that the cross section is independent of the choices of parametrization while the extracted terms are dependent on the choice. The bands structures closer to the bottom in the plots are the individual angular harmonics. This analysis and the parallel analysis agrees within error bars. KM15 model represents our data better than KM10

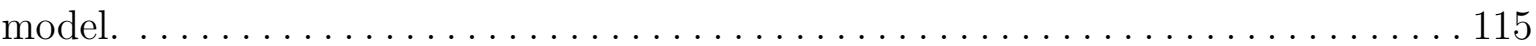


56 DVCS helicity dependent cross section for kin 361 with Beam energy $=7.38 \mathrm{GeV}$, $<Q^{2}>=3.2 \mathrm{GeV}^{2}$ and $\left\langle x_{B}\right\rangle=0.36$. Closed boxes/Filled colors represents Choice 1 given in section 7.2 .4 . Open boxes/ Polka Squares represents Choice 2 given in section 7.2.4. The Grey bands around the fits are the statistical uncertainty. Magenta dot represents a parallel analysis by Frédéric Georges[33]. Blue and Brown lines are two global fits given in [57, 56]. Agreement between the different parametrization validates the claim that the cross section is independent of the choices of parametrization. This analysis and the parallel analysis agrees to the most part. There are slight disagreement in the edges of the bin at $\phi=0^{0}$ or $\phi=360^{\circ}$. The Blue and Green bands are the individual angular harmonic terms. 116

57 Figure shows a comparison between two different choices of parametrization dis-

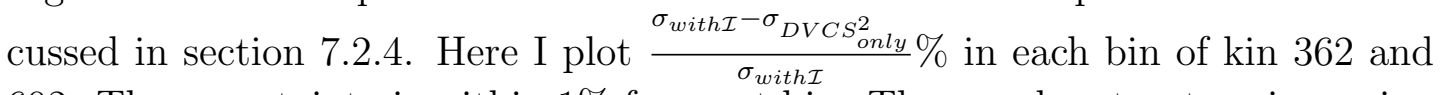
602. The uncertainty is within 1\% for most bin. The angular structure is coming from the angular dependency of the Bethe-Heitler propagators in the denominator

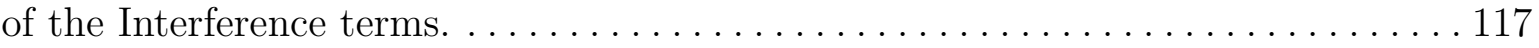

58 Figure shows a comparison between two parallel cross section extraction performed in this thesis and by Frédéric Georges in [33]. The two extractions are in agreement within the error bar except for the first $2 t_{\text {bins }}$ of kin 601 and 603 as

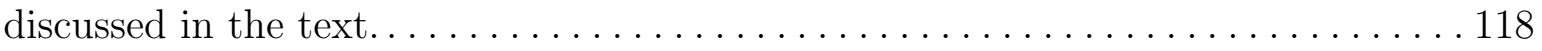

59 DVCS unpolarized cross section for Kin 361. Cross section is extracted with 2 different parametrization choices discussed in section 7.2 .4 . The bands around the fits are the statistical uncertainty. Magenta dot represents a parallel analysis

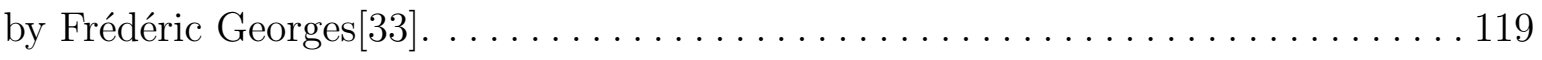

60 DVCS helicity dependent cross section for Kin 361. Cross section is extracted with 2 different parametrization choices discussed in section 7.2 .4 . The bands around the fits are the statistical uncertainty. Magenta dot represents a parallel

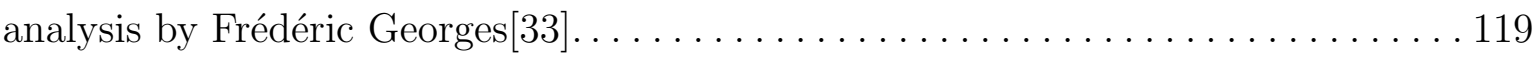

61 DVCS unpolarized cross section for Kin 362. Cross section is extracted with 2 different parametrization choices discussed in section 7.2 .4 . The bands around the fits are the statistical uncertainty. Magenta dot represents a parallel analysis by Frédéric Georges [33]. Model cross sections are given in [57] [56] . . . . . . . 120

62 DVCS helicity dependent cross section for Kin 362. Cross section is extracted with 2 different parametrization choices discussed in section 7.2 .4 . The bands around the fits are the statistical uncertainty. Magenta dot represents a parallel analysis by Frédéric Georges [33]. Model cross sections are given in [57] [56] . . . . 120 
63 DVCS unpolarized cross section for Kin 363. Cross section is extracted with 2 different parametrization choices discussed in section 7.2 .4 . The bands around the fits are the statistical uncertainty. Magenta dot represents a parallel analysis by Frédéric Georges [33]. Model cross sections are given in [57] [56] . . . . . . . . 121

64 DVCS helicity dependent cross section for Kin 363. Cross section is extracted with 2 different parametrization choices discussed in section 17.2 .4 . The bands around the fits are the statistical uncertainty. Magenta dot represents a parallel analysis by Frédéric Georges [33]. Model cross sections are given in [57] [56] . . . . 121

65 DVCS unpolarized cross section for Kin 481. Cross section is extracted with 2 different parametrization choices discussed in section 7.2 .4 . The bands around the fits are the statistical uncertainty. Magenta dot represents a parallel analysis by Frédéric Georges [33]. Model cross sections are given in [57] [56] . . . . . . . . 122

66 DVCS helicity dependent cross section for Kin 481. Cross section is extracted with 2 different parametrization choices discussed in section 7.2 .4 . The bands around the fits are the statistical uncertainty. Magenta dot represents a parallel analysis by Frédéric Georges [33]. Model cross sections are given in [57] [56] . . . . 122

67 DVCS unpolarized cross section for Kin 482. Cross section is extracted with 2 different parametrization choices discussed in section 7.2 .4 . The bands around the fits are the statistical uncertainty. Magenta dot represents a parallel analysis by Frédéric Georges [33]. Model cross sections are given in [57] [56] . . . . . . . . 123

68 DVCS helicity dependent cross section for Kin 482. Cross section is extracted with 2 different parametrization choices discussed in section 7.2 .4 . The bands around the fits are the statistical uncertainty. Magenta dot represents a parallel analysis by Frédéric Georges [33]. Model cross sections are given in [57] [56] . . . . 123

69 DVCS unpolarized cross section for Kin 483. Cross section is extracted with 2 different parametrization choices discussed in section 7.2 .4 . The bands around the fits are the statistical uncertainty. Magenta dot represents a parallel analysis by Frédéric Georges [33]. Model cross sections are given in [57] [56] . . . . . . . 124

$70 \quad$ DVCS helicity dependent cross section for Kin 483. Cross section is extracted with 2 different parametrization choices discussed in section 17.2 .4 . The bands around the fits are the statistical uncertainty. Magenta dot represents a parallel analysis by Frédéric Georges [33]. Model cross sections are given in [57] [56] . . . . 124

71 DVCS unpolarized cross section for Kin 484. Cross section is extracted with 2 different parametrization choices discussed in section 7.2 .4 . The bands around the fits are the statistical uncertainty. Magenta dot represents a parallel analysis by Frédéric Georges [33]. Model cross sections are given in [57] [56] . . . . . . . . 125 
72 DVCS helicity dependent cross section for Kin 484. Cross section is extracted with 2 different parametrization choices discussed in section 7.2 .4 . The bands around the fits are the statistical uncertainty. Magenta dot represents a parallel analysis by Frédéric Georges [33]. Model cross sections are given in [57] [56] . . . . 125

73 DVCS unpolarized cross section for Kin 601. Cross section is extracted with 2 different parametrization choices discussed in section 7.2 .4 . The bands around the fits are the statistical uncertainty. Magenta dot represents a parallel analysis by Frédéric Georges [33]. Model cross sections are given in [57] [56] . . . . . . . . 126

74 DVCS helicity dependent cross section for Kin 601. Cross section is extracted with 2 different parametrization choices discussed in section 7.2 .4 . The bands around the fits are the statistical uncertainty. Magenta dot represents a parallel analysis by Frédéric Georges [33]. Model cross sections are given in [57] [56] . . . . 126

75 DVCS unpolarized cross section for Kin 603. Cross section is extracted with 2 different parametrization choices discussed in section 7.2 .4 . The bands around the fits are the statistical uncertainty. Magenta dot represents a parallel analysis by Frédéric Georges [33]. Model cross sections are given in [57] [56] . . . . . . . 127

76 DVCS helicity dependent cross section for Kin 603. Cross section is extracted with 2 different parametrization choices discussed in section 7.2 .4 . The bands around the fits are the statistical uncertainty. Magenta dot represents a parallel analysis by Frédéric Georges[33]. Model cross sections are given in [57] [56] . . . . 127 


\section{CHAPTER 1}

\section{INTRODUCTION}

\subsection{UNDERSTANDING THE INTERNAL MAKEUP AND STRUCTURE OF PROTON}

Through the many attempts to understand the nature of matter, from five fundamental elements of ancient greek philosophy to Medeleev's Periodic Table, we are interested in answering the question: "What are we made up of, and How?". With the advent of particle accelerators and electron-proton scattering, our understanding of what are we made up of has evolved a lot in the past half a century. The question of "How", however is another story.

The Standard Model of particle physics defines quarks and leptons as the basic building blocks of all matter. The interaction between them are mediated by force carrying gauge bosons. Leptons can occur freely in nature and their interactions are well established by the Electro-Weak theory. Quarks on the other hand, are confined and observable only in the subatomic particles collectively called hadrons (protons, neutrons, atomic nuclei, and the mesons, e.g. pions, kaons). Quantum Chromo-Dynamics (QCD) is the theory governing the strong interaction between quarks and the force carrier for strong interactions: gluons. As powerful as QCD is, our understanding is not complete enough to derive many of the basic physical observables of hadrons from the fundamental quark-gluon degrees of freedom. To bridge the gap, theorists use experimentally accessible densities functions. These densities functions provide insight into the interactions of a quark-gluon system.

From the late 50's, two such densities functions are extensively used to provide information about a hadron.

- Late 50's : Form Factors, accessible via elastic scattering of electron and proton, gave us insight into the electric and magnetic charge distribution [43]

- Late 60's : Structure Functions, accessible via Deep Inelastic Scattering of electron and proton, describes the longitudinal momentum density of quarks and gluons in the hadronic system 62 
In the mid 90's, theorists developed a new kind of densities function, called Generalized Parton Distributions (GPDs) which are shown to combine the information contained in both Form Factors and Structure Functions. The GPDs provide, in particular, information on the correlated transverse spacial and longitudinal momentum distributions.

The electron, a lepton, is conjectured to be a fundamental particle, as there is no known internal structure. Interactions of electrons with other charged particles are well described by Quantum Electro-Dynamics (QED). To see the internal structure of a hadron, one needs a probe with wavelength smaller than the size of a hadron. Louise De Broglie in his PhD thesis [58] hypothesized that an electron has a wavelength inversely proportional to its momentum

as $\lambda=\frac{\hbar}{P}$. Thus experimentalists use relativistically accelerated electrons as a probe to examine the internal structure of hadrons. A hydrogen ion contains only one proton and hence is the most convenient target to study a system of quarks. Therefore, electron-proton scattering is used to access a whole set of useful insights into the dynamics of strongly interacting systems. To access GPDs, one need to look into deep exclusive processes, where the target hadron is violently excited, but only a minimal number of final state particles are produced. Deeply Virtual Compton Scattering (DVCS), an $e p \rightarrow e p \gamma$ process, is the simplest and cleanest such process. It was shown successfully, in previous generations of experiments that, GPD's can be accessed via DVCS [19, 4, 26].

\subsection{EXPERIMENT OVERVIEW}

CEBAF accelerator of Thomas Jefferson Nation Accelerator Facility (JLab) in Newport News, Virginia is one of a kind accelerator capable of providing continuous electron beam, accelerated up to $12 \mathrm{GeV}$. A pair of High Resolution Spectrometers (HRS) located in Experimental Hall A of JLab provides a comprehensive detector package capable of detecting electrons while rejecting pions at higher rate with a very focused acceptance, allowing experimentalists to achieve high luminosity of the order of $10^{38} \mathrm{~cm}^{-2} \mathrm{~s}^{-1}$. The setup of the Hall A also has the flexibility of installing additional detectors necessary for specific experiments, in the case of DVCS experiments a $\mathrm{PbF}_{2}$ calorimeter for photon detection located closer to the target and beam line. The DVCS Working Group at Hall A, JLab has successfully conducted 3 generations of experiments dedicated to access GPD's and test various properties of these new kind of densities functions via the extraction of the DVCS cross-section. 


\subsection{ANALYSIS OVERVIEW}

This thesis extracts DVCS cross section from the $3^{\text {rd }}$ generation DVCS experiment (DVCS3) conducted from Fall 2014 to Fall 2016 at Hall-A, JLab. This thesis uses a fitting method to extract the cross-section from the number of DVCS event found in the experiment (yield). To get the yield, one must first identify a DVCS event from all possible competing events of the electron-proton scattering. A DVCS event is characterized by

- A good single electron in the final state : Final state electrons are observed in Left-HRS. A threshold Gas Cherenkov and a Pb-Glass Calorimeter called the Pion Rejector provides the Electron ID. A Vertical Drift Chamber provides the tracking information and used to select only events with one electron in the final state. A set of Rvachev Functions ensures the electron acceptance. A Target cut makes sure the electron observed is, in fact originated from the liquid hydrogen target.

- A good photon in the calorimeter : DVCS3 uses a custom calorimeter to identify final state photons. Due to the high event rate, an Analogue Ring Sampler (ARS) is used to sample the photons. A wave form analysis is performed to extract the amplitude and the arrival time of the ARS signal as well as to deal with the pile-up due to the high rate of accidentals. A clustering algorithm then finds individual photons. An $e p \rightarrow e p \pi^{0}$ event can sometimes deposit only one of the two decay photon from $\pi_{0}$ in the calorimeter due to it's finite acceptance. A statistical subtraction procedure called $\pi^{0}$ subtraction is performed to handle this. A coincidence time window between the detected electron in HRS and the photon in DVCS Calorimeter will make sure the electron and photon detected are in fact from the same event. Finally accidental events due to random coincidences can be subtracted by estimating the events in a timing window outside of the main coincidence window.

- Exclusivity using missing mass : In addition to an electron and a photon, DVCS event will have a proton in the final state. DVCS3 does not detect the final state proton, instead the exclusivity is ensured by requiring the mass of the undetected particle or particles as inferred from the detected particles to be close to that of a proton. This technique is also extended to find the single DVCS photon when the clustering algorithm registers more than one photon in the calorimeter. 
The yield obtained should be corrected for the efficiencies of each step involved in the event selection.

- Trigger Efficiency : DVCS3 data acquisition happens only when there is a signal in the S2 scintilator array, Gas Cherenkov and the DVCS Calorimeter in coincidence. Any inefficiencies of the trigger system in identifying a possible event will affect its ability to record such events.

- Dead time correction : Data acquisition system can sometimes miss to record some events when it was busy writing a batch of previous events to the disk from memory, due to the limitations of the system. This time in which the DAQ misses to register events is accounted for by the live time correction factor.

- Multi Track Correction : Due to some shortcomings of the VDC tracking algorithm and a large amount of low energy electron showering, roughly $10 \%$ of the events have ambiguous tracking data with multiple track candidates. Good single track electron candidates among these corrupted events are accounted for as a correction.

\subsection{CROSS-SECTION EXTRACTION METHOD}

DVCS Cross-Section is parametrized from QCD theory in a Fourier harmonic expansion described in BMK model[14].

$$
d \sigma=\sum_{j \in C F F_{t e r m s C_{j}}} K_{j}\left(E, Q^{2}, \chi_{B}, t, \phi_{\gamma \gamma}, \phi_{e}\right) \times C_{j}(t)
$$

Where, $C_{j} \in\left\{C_{n}^{T}, S_{n}^{T} \mid T=D V C S, B H, \mathcal{I} ; n=0 \ldots 3\right\}$, are angular harmonics with kinematic factors $K_{j}$. BH, Bethe-Heitler is a competing ep $\rightarrow e p \gamma$ process, were unlike in the DVCS process, the photon in the final state is emitted by the electron instead of the proton. $C_{j}^{B H}$ are bi-linear combinations of Elastic Form Factors and exactly calculable. $C_{j}^{D V C S}$ are bi-linear combinations of complex integrals called Compton Form Factors (CFF) connecting the DVCS Cross Section to GPDs. $C_{j}^{\mathcal{I}}$ are from the interference between DVCS and BH, and are linear combinations of both Elastic and Compton form factors. DVCS cross section is reconstructed from the free parameters $C_{j}$ extracted by fitting the experimental yield in the $k^{t h}$ bin $N_{k}^{e x p}$ to the simulated number of events in the same bin $N_{k}^{s i m}$ by minimizing the $\chi^{2}$

$$
\chi^{2}=\sum_{k=1}^{N_{b i n}}\left(\frac{N_{k}^{e x p}-N_{k}^{s i m}}{\sigma_{k}}\right)^{2}
$$


Two types of cross-sections are extracted based on beam helicity, either is sensitive to the GPD's in different ways. To extract helicity dependent cross section, beam helicity is flipped with a $30 \mathrm{~Hz}$ frequency. A helicity cut and a correction associated with it are also involve in the helicity dependent cross section.

\subsection{THESIS ORGANIZATION}

- Chapter 1, this current chapter provided an extended summary and overview of the thesis introducing the basics of QCD, GPDs, CFF, DVCS, 12 GeV DVCS experiment at Hall-A JLab and the analysis procedure.

- Chapter 2 presents the theoretical framework for Generalized Parton Distributions, Compton Form Factors and the connection between DVCS Cross-Section and GPD's. It also provides more context to the DVCS experiments in the large scale of high energy physics.

- Chapter 3 covers the experimental procedure and instrumentation. It describes the experimental process, setup, detector packages and data acquisition system.

- Chapter 4 describes the electron selection in the HRS including the calibration of HRS detectors and efficiency analysis.

- Chapter 5 summarizes the spectrometer normalization studies explaining charge calculation, radiative correction, deadtime correction and the DIS trigger analysis.

- Chapter 6 presents the DVCS Calorimeter analysis for photon selection and MonteCarlo simulation. It covers the details of calorimeter clustering, $\pi^{0}$ subtraction, accidental subtraction and multi-cluster correction.

- Chapter 7 describes the cross section extraction procedure and presents the preliminary cross section results and some discussions. 


\section{CHAPTER 2}

\section{PROBING A PROTON THROUGH ELECTRON SCATTERING EXPERIMENTS}

In humanity's very long quest for understanding the make-up of matter, after the discovery of radioactivity[11] and electron [35] in the last decade of $19^{\text {th }}$ century, and Rutherford's gold foil experiment [72] in the early decades of $20^{\text {th }}$ century an important breakthrough came when humanity acquired the ability to accelerate electrons. This enabled experimentalists to bombard hadrons with higher and higher energy particles and study various aspects of them. In scattering experiments a stationary subject can be bombarded by a structure-less particle or two particles can be collided together in a head-on collision. The first case is called fixed target experiments and has the advantage of high luminosity, where the number of occurrences of a scattering is high, but since only the probe is accelerated the available center of mass energy in the scattering is low. The second case is called a collider experiment where the available center of mass energy is large due to the acceleration of both particles, but the luminosity is low. We will limit our discussion in this thesis to the fixed target experiments. The merits of the electron-proton fixed target scattering as a tool to study the internal structure of a hadron is discussed in section 1.1. Depending on the energy available in the reaction, the outcome of the reaction would differ. The scattering process, then can be subdivided in to two categories providing different information about the struck hadron.

\subsection{ELASTIC SCATTERING}

When the final state of the scattering is composed only of the initial scattering particles, the scattering is called an Elastic Scattering. In these experiments all final state particles are detected to ensure they are the same as the initial particles. These types of experiments are called exclusive processes since it was ensured that the process occurred in the experiment is only the considered one.

Figure 1 shows a Feynman diagram of an elastic $e p \rightarrow e^{\prime} p^{\prime}$ scattering in the single photon exchange approximation. Elastic cross section for a relativistic electron probe and a pointlike particle is given by the Mott cross section as follows,

$$
\left(\frac{d \sigma}{d \Omega}\right)_{M o t t}=\frac{\alpha^{2}}{4 E^{2} \sin ^{4}\left(\frac{\theta}{2}\right)}\left(\frac{E}{E^{\prime}}\right) \cos ^{2}\left(\frac{\theta}{2}\right)
$$




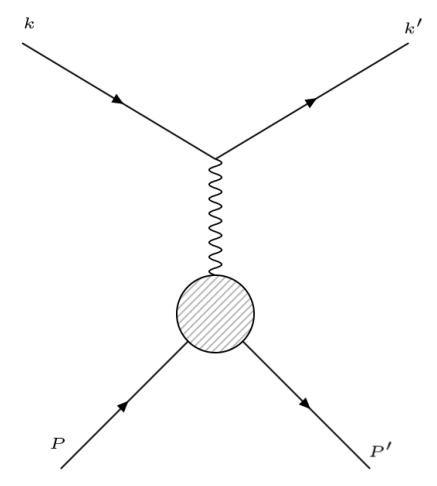

FIG. 1: Figure shows a Feynman diagram of an elastic $e p \rightarrow e^{\prime} p^{\prime}$ scattering in the single photon exchange approximation. Incoming electron emits a virtual photon in the presence of a proton and scatters off. The virtual photon is absorbed by the proton and the proton momentum is changed as a result. $k, k^{\prime}, P$ and $P^{\prime}$ are the four-vectors of incoming electron, scattered electron, initial proton and the final proton.

From the Rosenbluth formalism[71], by giving the charge and the magnetic moment phenomenological interpretations instead of the meson field correction, the scattering cross section for a point charge with anomalous magnetic moment $\mu$ can be given as follows [61],

$$
\begin{aligned}
\sigma & =\sigma_{N S}\left\{1+\frac{Q^{2}}{4 M^{2}}\left[2(1+\mu)^{2} \tan ^{2}\left(\frac{\theta}{2}\right)+\mu^{2}\right]\right\} \\
\sigma_{N S} & =\frac{e^{4}}{4 E^{2}}\left(\frac{\cos ^{2}\left(\frac{\theta}{2}\right)}{\sin ^{4}\left(\frac{\theta}{2}\right)}\right) \frac{1}{1+\frac{2 E}{M} \sin ^{2}\left(\frac{\theta}{2}\right)}
\end{aligned}
$$

where $\mathrm{M}$ is the rest mass of the proton, $\mu=1.79 \ldots$ for the proton and $Q^{2}$ is the invariant momentum transfer in the laboratory frame given as,

$$
Q^{2}=-q^{2}=-\left(k-k^{\prime}\right)^{2}=4 E E^{\prime} \sin ^{2}\left(\frac{\theta}{2}\right) .
$$

When R. Hofstadter et al. 61], performed elastic scattering on a proton with $188-\mathrm{MeV}$ electron in 1950, they discovered that the measured cross section fell below the prediction for a point particle with an anomalous magnetic moment given in equation 4 . This indicated that the proton actually is not a point-like particle, but rather has a form. For a diffused proton, the proton with a form, this formulae can be modified by introducing two Form Factors $F_{1}$ and $F_{2}$ as follows,

$$
\sigma=\sigma_{N S}\left\{F_{1}^{2}+\frac{q^{2}}{4 M^{2}}\left[2\left(F_{1}+\mu F_{2}\right)^{2} \tan ^{2}\left(\frac{\theta}{2}\right)+\mu^{2} F_{2}^{2}\right]\right\}
$$




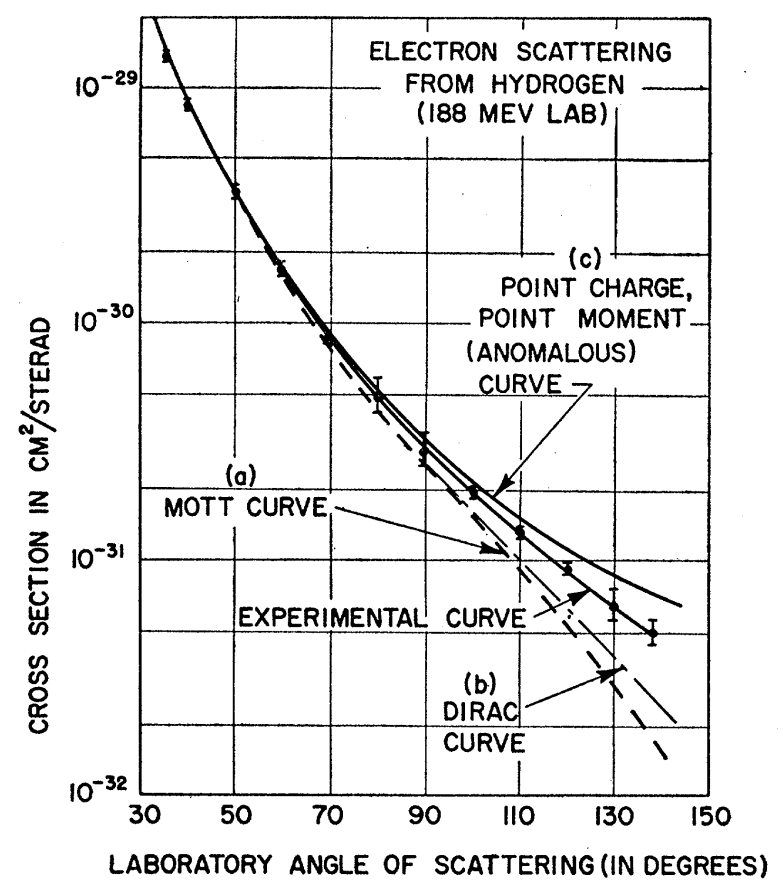

(a) Elastic Cross Section

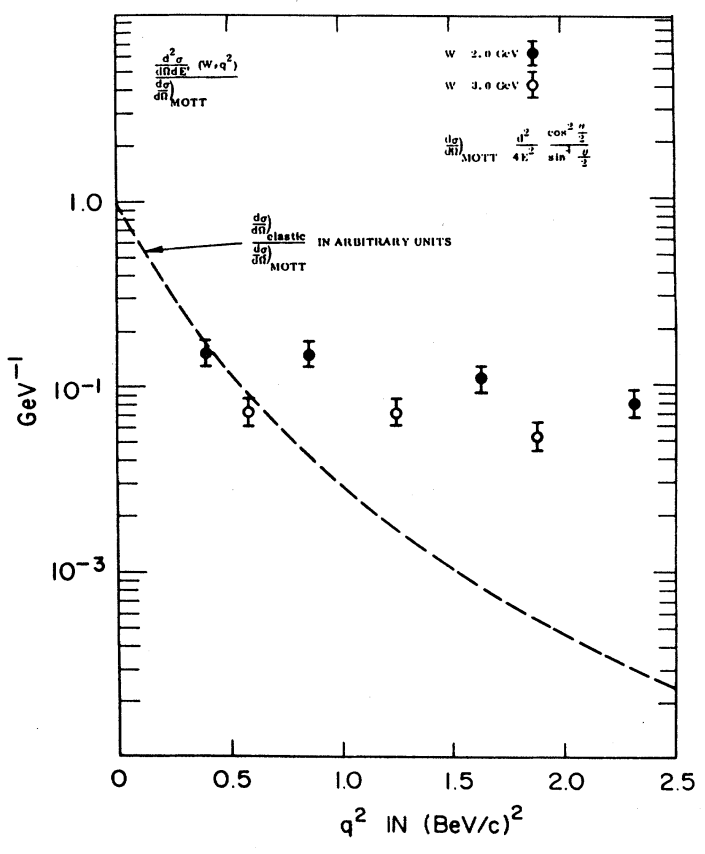

(b) Deep Inelastic Cross Section.

FIG. 2: Figure 2a gives the measured elastic cross section as a function of scattering angle from [61]. The measured cross section deviated from the prediction for a point-like particle (equation 3), point charge(5) and point charge with anomalous magnetic moment (4) indicating a diffused structure for the proton. Figure $2 \mathrm{~b}$ gives the ratio of measured DIS cross section to the Mott cross section with $Q^{2}$ for different values of the invariant mass $W$ from [54].The dotted curve indicates the prediction for the ratio as inferred from previous experiments with low beam energy. The open $(\mathrm{W}=3 \mathrm{GeV})$ and closed $(\mathrm{W}=2 \mathrm{GeV})$ circle points following a straight-line indicating that the electrons are in fact scattering off of a point-like particle within the proton. These particles are called Partons at the time and later identified with the quarks suggested by Gell-Mann[36] and Zweig[80] 
$F_{1}$ is the charge form factor known as Dirac form factor and $F_{2}$ is the anomalous magnetic moment form factor known as Pauli form factor and they depend on $Q^{2}$. A more recognizable form of these form factors are given as,

$$
\begin{aligned}
G_{E}\left(Q^{2}\right) & =F_{1}\left(Q^{2}\right)-\tau \mu F_{2}\left(Q^{2}\right) \\
G_{M}\left(Q^{2}\right) & =F_{1}\left(Q^{2}\right)+\mu F_{2}\left(Q^{2}\right) \\
\tau & =\frac{Q^{2}}{4 M} .
\end{aligned}
$$

The $Q^{2}$ evolution of the form factors are connected to the charge and magnetic moment distribution within the proton as $G_{E}\left(Q^{2}\right)=1-\frac{1}{6}<r_{E}^{2}>Q^{2}+\mathcal{O}\left(Q^{4}\right)$ etc.. At $Q^{2}=0$ the charge and magnetic radii of the proton can be defined as,

$$
\begin{aligned}
& <r_{E}^{2}>=-6 \frac{G_{E}\left(Q^{2}\right)}{d Q^{2}} \\
& <r_{M}^{2}>=\frac{-6}{G_{M}(0)} \frac{G_{M}\left(Q^{2}\right)}{d Q^{2}}
\end{aligned}
$$

\subsection{DEEP INELASTIC SCATTERING}

When the electron carries high enough energy to break the proton apart, the final state particles would not be the same as the initial particles. These types of scattering are called inelastic scattering. When the energy is high enough for the electron to penetrate the proton and interact with a sub structure, we add an adjective of "Deep". In these types of processes, usually many many final state particles are produced and it would be virtually impossible to detect all of them. When only the scattered electron is detected ensuring the process happened, in fact involved the scattering of the electron and the electron energy loss is high, this process is called Deep Inclusive Scattering or Deep Inelastic Scattering (DIS). Sometimes in addition to the scattered electron, one or more other final state particles are detected to ensure the occurrences of additional processes. These types of experiments are called Semi Inclusive Scattering or Semi Inclusive Deep Inelastic Scattering (SIDIS).

Figure 3 shows a Feynman diagram of Deep Inelastic Scattering in the single photon exchange approximation: $k, k^{\prime}$, and $P$ are the four-vectors of incoming electron, scattered electron and initial proton and $X$ is the sum of all possible hadronic final states, which are not detected. The only information about this comes from the invariant mass, defined as,

$$
\begin{aligned}
W^{2} & =\left(p+k-k^{\prime}\right)^{2}=2 M \nu-Q^{2}+M^{2} \\
\nu & =E-E^{\prime},
\end{aligned}
$$




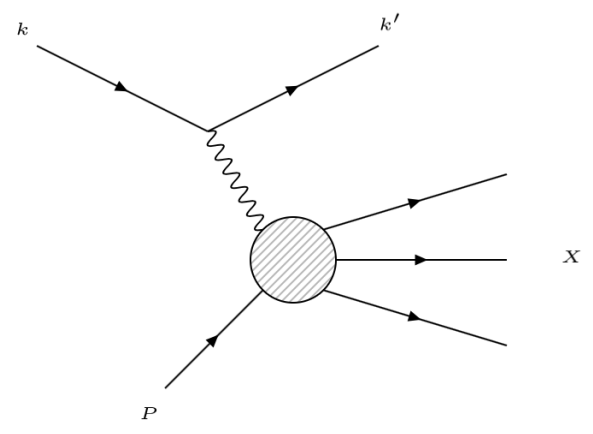

(a) Inclusive Inelastic Scattering

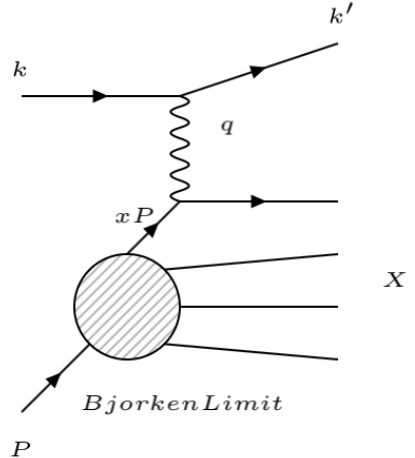

(b) DIS in Bjorken Limit

FIG. 3: Figure 3a shows a Feynman diagram of an inelastic $e p \rightarrow e^{\prime} X$ scattering in the single photon exchange approximation. Incoming electron emits a virtual photon in the presence of a proton and scatters off. The virtual photon interacts with the proton and breaks it apart. $k, k^{\prime}$, and $P$ are the four-vectors of incoming electron, scattered electron and initial proton and $X$ is the sum of all possible hadron final states. Figure $3 \mathrm{~b}$ shows the Feynman diagram of DIS in the Bjorken limit where the virtual photon scatters of of a parton inside the proton carrying a fractional momentum $x$ of the proton.

where $\mathrm{E}$ is the energy of the incoming electron and $\mathrm{E}$ ' is the energy of the scattered electron. For elastic scattering, $\nu=E-E^{\prime}=\frac{Q^{2}}{2 M}$. When R. Hofstadter et al. [61] performed their famous experiment discussed in section 2.1 they also found that the elastic cross section decreased with increasing $Q^{2}$. When they used electron beam with energies up to $550 \mathrm{MeV}$ they noticed that many new particles emerged in the final state. All these particles were initially thought to be composites of each other with no "fundamental" particle [54, 20] in the theory known as nucleon democracy. Gell-Mann [36] and George Zweig [80] independently proposed a model based on fundamental particle with spin $\frac{1}{2}$ and fractional electric charge called quarks. This model was able to reproduce the SU(3) symmetries of the observed hadron spectrum with high accuracy [54]. After the establishment of the Stanford Linear Accelerator, the possibility of accelerating electron beyond a few $\mathrm{GeV}$ became a reality and MIT-SLAC collaboration [62] performed Deep Inelastic Scattering (DIS) experiments with up to $21 \mathrm{GeV}$ electron beam. They measured the cross section in various scattering angles and found that the ratio of $\sigma / \sigma_{M o t t}$ became uniform and independent of $Q^{2}$ for a given value of $\mathrm{W}$ given in equation 13 indicating point-like constituents for the proton. 


\subsubsection{SCALING AND FEYNMANS PARTON MODEL}

Similar to the Elastic cross section, DIS cross section is parametrized by two structure functions $W_{1}\left(\nu, Q^{2}\right)$ and $W_{2}\left(\nu, Q^{2}\right)$,

$$
\frac{d \sigma}{d \Omega d E^{\prime}}=\sigma_{M o t t}\left\{W_{2}\left(\nu, Q^{2}\right)+2 W_{1}\left(\nu, Q^{2}\right) \tan ^{2}\left(\frac{\theta}{2}\right)\right\}
$$

$W_{2}\left(\nu, Q^{2}\right)$ dominates the cross section at smaller angles and $W_{2}\left(\nu, Q^{2}\right)$ controls the large angle scattering. J. D. Bjorken observed that in the limits of $Q^{2} \rightarrow \infty$ both $W_{1}$ and $W_{2}$ becomes a function only of a variable $\omega=\left(W^{2}+Q^{2}-M^{2}\right) / Q^{2}=\frac{2 M \nu}{Q^{2}}$ [17]. Kendall [68] observed scaling in a study of $\nu W_{2}$ as a function of $\omega$, where $\nu W_{2}$ became only a function of $\omega$ independent of $Q^{2}$ and $\nu$. Richard Fynman explained the phenomena using his constituent model for the hadron [31, 68, 54], where the constituents were referred to as partons. He explained that in the infinite momentum frame, where the electron is slowed to a stand still and the proton is moving towards it, under the impulse approximation, where the short-range interactions between the partons are neglected while the virtual photons are exchanged, DIS becomes the coherent sum of the elastic scattering of electrons, off of these "free" partons, thus the $\nu W_{2}$ reflects the dynamics of the free partons. This limit in which the partons behave as if they are free is called the Bjorken limit and the phenomena is later identified as a consequence of QCD asymptotic freedom. $1 / \omega$ is now referred to as Bjorken- $x\left(x_{B}\right)$ In this limit, the variable $x_{B}$ can be shown as the fractional momentum carried by the struck parton. Figure $3 \mathrm{~b}$ shows the Feynman diagram of DIS in the Bjorken limit. The modern form of the functions $W_{1}$ and $W_{2}$ are given as follows,

$$
\begin{aligned}
F_{1}\left(x_{B}\right) & =2 M W_{1}\left(\nu, Q^{2}\right) \\
F_{2}\left(x_{B}\right) & =\nu W_{2}\left(\nu, Q^{2}\right) \\
F_{2} & =2 x_{B} F_{1}
\end{aligned}
$$

The functions $F_{2}\left(x_{B}\right)$ are related to the Parton Distribution Function (PDF) in the asymptotic region as,

$$
F_{2}(x)=\sum_{i \in \text { parton }} e_{i}^{2} x q_{i}(x)
$$

where, $e_{i}$ is the charge of the parton $i$ and $q_{i}(x)$ are the PDF's giving the distribution of parton $i$ with the longitudinal momentum fraction $x$. These partons are later identified as the quarks proposed by Gell-Mann and Zweig. 


\subsubsection{SCALING VIOLATION AND QCD IMPROVED PARTON MODEL}

Global fits to the DIS data over large ranges of $x_{B}$ revealed that the scaling observed in the initial DIS experiments are violated for both $x_{B} \ll 1$ and $1-x_{B} \ll 1$ regions [55]. This phenomena is explained by the existence of gluons in the hadron. Quarks interact via exchange of gluons. In higher $x_{B}$ region, where the struck quark carrying most of the proton momentum, quarks can radiate gluons and lose their momentum thus reducing the density. In lower $x_{B}$ region, where most of the momentum of the proton is carried by the gluon, gluons can split creating more quark anti-quark pairs thus increasing the density of the quarks. These radiations are given by Dokshitzer-Gribov-Lipatov-Altarelli-Parisi (DGLAP) evolution [7]. In this QCD improved parton model, we have quarks and gluons as partons and any given hadronic system can have large number of quarks satisfying the sum rule $\sum_{f} q_{f}-\overline{q_{f}}=n_{f}^{V}$.

For a proton, these sum rules are

$$
\begin{aligned}
& \sum_{u} q_{u}-\overline{q_{u}}=2 \\
& \sum_{d} q_{d}-\overline{q_{d}}=1 \\
& \sum_{s} q_{s}-\overline{q_{s}}=0,
\end{aligned}
$$

where $u$ is up quark, $d$ is down quark, and $s$ is for strange quark. For each quark flavor $f$ the valence and sea distributions are defined as $q_{\text {Valence }}=q_{f}-\bar{q}_{f}$ and $q_{\text {sea }}=q_{f}+\bar{q}_{f}$. However, these definitions do not have meaning as probability distributions.

\subsection{GENERALIZED PARTON DISTRIBUTIONS}

In section 2.1 we discussed elastic form factors and the charge distribution of a proton, accessed via exclusive elastic scattering. In section 2.2 we discussed parton distribution functions and the longitudinal momentum distribution accessed via deep inelastic scattering. These two processes provide us with complementary information but from two independent methods without any correlation. Generalized Parton Distributions developed in mid 90s by Muller et al. [64], A.V Radyushkin [67] and X. Ji [47], provide correlated transverse-spacial and longitudinal-momentum distributions. 


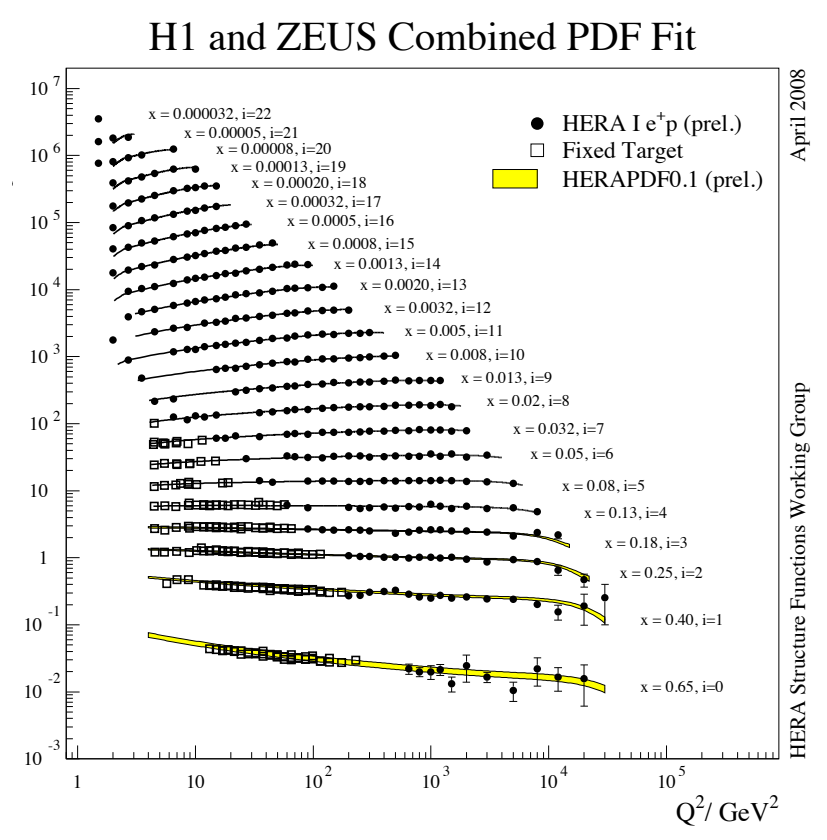

(a) Scaling violation

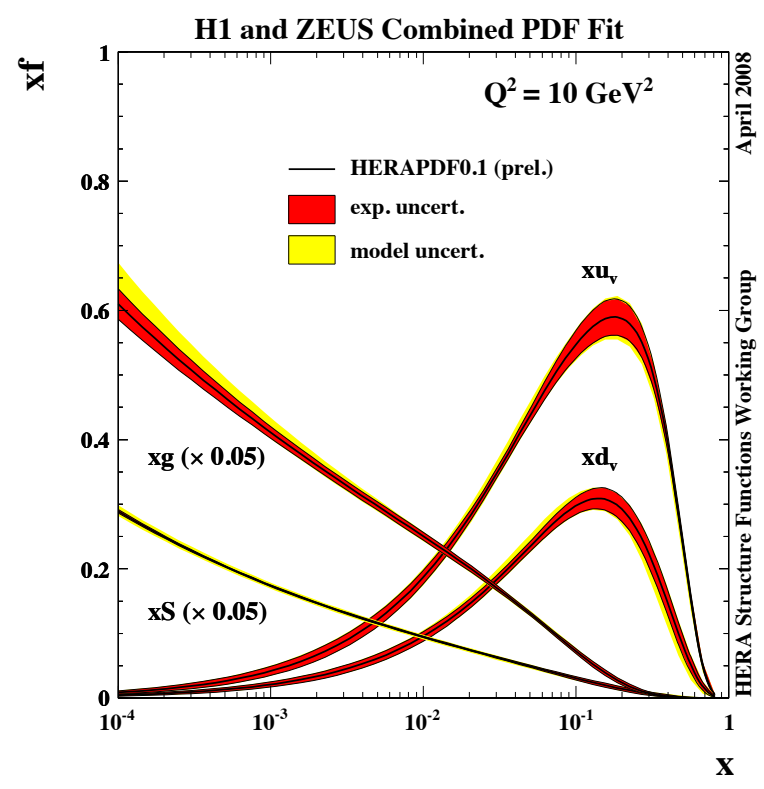

(b) Valence quark distribution

FIG. 4: Figure 4a shows the global fit of $F_{2}(x)$ vs $Q^{2}$ for various fixed $x_{B}$ values from [55]. Scaling is observed for $0.032 \leq X_{B} \leq 0.25$ region. For smaller $x_{B}$ values $F_{2}$ increases with $Q^{2}$ and for larger $x_{B}$ values $F_{2}$ decreases with increasing $x_{B}$ violating the scaling. This variation is explained by DGLAP evolution [7] discussed in section 2.2.2. Figure 4b shows the global fit for the valence distribution with $x$ for $Q^{2}=10 \mathrm{GeV}$. The plot shows the existence of twice as large up quark distribution than down quark distribution in the valence region. We also see that the valence quark distribution becomes much smaller in the small $x$ region while the gluon and the sea quark distribution rises as if the proton is made up of a soup of gluons, quarks and anti-quarks of all flavors. 


\subsubsection{QCD MATRIX ELEMENTS}

Define QCD matrix elements [15],

$$
<P^{\prime}\left|\bar{\psi}_{q}\left(y^{\prime}\right) \mathcal{O} \psi_{q}(y)\right| P>
$$

Here $\psi_{q}(y)$ is a quark field of flavor $q$ at a point $y$ in space-time. $P$ is the initial nucleon momentum, and $P^{\prime}$ is the final nucleon momentum. $\mathcal{O}$ is an operator which can be decomposed on the basis of 16 independent matrices of Dirac's theory [38]. These matrix elements are amplitudes of finding/creating a quark at point $y$ in space-time in a nucleon of momentum $P$ and placing-it/annihilating-it at another point $y^{\prime}$ in space-time in a nucleon of momentum $P^{\prime}$. Based on the values of $y, y^{\prime}$ and $P, P^{\prime}$ we have the following cases:

\section{Local, non-forward matrix elements}

If we look at the elastic scattering in Figure 1, we can conclude that only one point in space-time is involved, and the probed nucleon changed it's momentum by absorbing the virtual photon. The QCD matrix element for this process can be written as $<P^{\prime}\left|\bar{\psi}_{q}(0) \mathcal{O} \psi_{q}(0)\right| P>$. This is called a local, non-forward matrix element. These matrix elements are connected to the form factors discussed in section 2.1 as,

$$
<P^{\prime}\left|\bar{\psi}_{q}(0) \gamma^{+} \psi_{q}(0)\right| P>=F_{1}(-t) \bar{N}\left(P^{\prime}\right) \gamma^{+} N(P)+F_{2}(-t) \bar{N}\left(P^{\prime}\right) i \sigma^{+\nu} \frac{\Delta_{\nu}}{2 m_{N}} N(P)
$$

Here, $F_{1}(-t)$ and $F_{2}(-t)$ are the Dirac and Pauli form factors and $t=\Delta^{2}=\left(P^{\prime}-P\right)$ is the four-momentum transfer at the nucleon vertex. In the case of elastic scattering $-t$ will be $Q^{2}$

\section{Non-local, forward matrix elements}

If we look at the deep inelastic scattering in Figure 3b, we see that the struck quark was picked up at one point in space-time and put back at a different point in space-time with the constraint $\left(y^{\prime}-y\right)^{2}=0$. We don't detect the final state except for the scattered electron, and integrate over all possible final states yielding a symmetrical process with the same initial nucleon with the same momentum. We can write the square of QCD matrix element for this process as $\left\langle P\left|\bar{\psi}_{q}(0) \mathcal{O} \psi_{q}(y)\right| P>\right.$. This is called a non-local, forward matrix element. Fourier transform of the vector matrix element will give the parton distribution function discussed in section 2.2

$$
q(x)=\frac{P^{+}}{4 \pi} \int d y^{-} e^{i x P+y-}<P\left|\bar{\psi}_{q}(0) \gamma^{+} \psi_{q}(y)\right| P>\left.\right|_{y^{+}=\vec{y}_{\perp}=0}
$$




\section{Non-local, non-forward matrix elements}

The generalization of these operators of prime importance to this work are the non-local non-forward matrix elements [64, 67, 47], where the quark is picked up from one point in space-time and put back at another point in space-time while the nucleon remains intact but its momentum changes. This means the nucleon should be shaken vigorously for the quark to absorb a violent collision and then almost immediately radiate nearly all this energy either in the form of a photon or hadronic particle, while leaving the nucleon intact in the final state. Deeply Virtual Compton Scattering is such a process, discussed in detail in section 2.4. These off-diagonal matrix elements can be written as,

$$
\left\langle P^{\prime}\left|\bar{\psi}_{q}(0) \mathcal{O} \psi_{q}(y)\right| P\right\rangle
$$

\subsubsection{GENERALIZED PARTON DISTRIBUTION}

The Fourier transformation of the vector and axial elements of the equation 26 will give,

$$
\begin{aligned}
& \left.\quad \frac{P^{+}}{2 \pi} \int d y^{-} e^{i x P+y-}\left\langle P^{\prime}\left|\bar{\psi}_{q}(0) \gamma^{+} \psi_{q}(y)\right| P\right\rangle\right|_{y^{+}=\vec{y}_{\perp}=0} \\
& =H^{q}(x, \xi, t) \gamma^{+} N(P)+E^{q}(x, \xi, t) i \sigma^{+\nu} \frac{\Delta_{\nu}}{2 m_{N}} N(P) \\
& \left.\frac{P^{+}}{2 \pi} \int d y^{-} e^{i x P+y-}\left\langle P^{\prime}\left|\bar{\psi}_{q}(0) \gamma^{+} \gamma^{5} \psi_{q}(y)\right| P\right\rangle\right|_{y^{+}=\vec{y}_{\perp}=0} \\
& =\tilde{H}^{q}(x, \xi, t) \gamma^{+} \gamma^{5} N(P)+\tilde{E}^{q}(x, \xi, t) i \gamma^{5} \frac{\Delta^{+}}{2 m_{N}} N(P)
\end{aligned}
$$

Here, $H^{q}(x, \xi, t), E^{q}(x, \xi, t), \tilde{H}^{q}(x, \xi, t)$ and $\tilde{E}^{q}(x, \xi, t)$ are called Generalized Parton Distributions. $\xi$ is the skewness. $x+\xi$ is the longitudinal momentum fraction carried by the struck quark and $x-\xi$ is the longitudinal momentum fraction carried by the quark returning to the final state, making $-2 \xi$ the longitudinal momentum transfer. $t=\Delta^{2}=\left(p^{\prime}-p\right)^{2}$ is the total 4 -momentum transfer squared at the nucleon vertex. $\Delta$ has a perpendicular component in addition to the $-2 \xi$.

\section{Properties of Generalized Parton Distributions}

\section{Correlation of transverse position and longitudinal momentum distributions}

When $\xi=0$ and $t=0$ there is no momentum transfer to the nucleon. Then the QCD matrix elements will look like non-local forward matrix elements, giving,

$$
\begin{array}{ll}
H^{q}(x, 0,0)=q(x), & \bar{H}^{q}(x, 0,0)=x \Delta q(x) \\
H^{g}(x, 0,0)=g(x), & \bar{H}^{g}(x, 0,0)=x \Delta g(x)
\end{array}
$$


Where $q(x)$ and $\Delta q(x)$ are unpolarized and polarized Parton Distribution Functions. We also get two polarized and unpolarized gluon density functions $g(x)$ and $\Delta g(x)$,

At finite momentum transfer, following sum rules will relate the elastic form factors to the GPDs

$$
\begin{aligned}
& \int_{-1}^{1} d x H^{q}(x, \xi, t)=F_{1}^{q}(t), \quad \int_{-1}^{1} d x E^{q}(x, \xi, t)=F_{2}^{q}(t), \\
& \int_{-1}^{1} d x \bar{H}^{q}(x, \xi, t)=G_{A}^{q}(t), \quad \int_{-1}^{1} d x \bar{E}^{q}(x, \xi, t)=G_{P}^{q}(t),
\end{aligned}
$$

Here $F_{1}^{q}(t)$ and $F_{2}^{q}(t)$ are Dirac and Pauli Form Factors. $G_{A}^{q}(t)$ and $G_{P}^{q}(t)$ are axial and induced pseudo scaler form factors.

\section{Quark orbital angular momentum}

Proton spin measured by the Spin Muon Collaboration (SMC) at CERN via polarized Deep Inelastic Scattering [3], showed that only about a $20 \%$ of the total spin of the proton is carried by the quark. Ji [48] first showed that the spin of the proton can be decomposed as follows.

$$
\begin{gathered}
J=J_{q}+J_{g}=\frac{1}{2} \\
J_{q}=\frac{\Delta \Sigma}{2}+L_{q}
\end{gathered}
$$

Here, $J_{g}$ is the spin contribution from gluons and $J_{q}$ is the contribution from quarks. $\frac{1}{2} \Delta \Sigma$ is the quark intrinsic spin measured by SMC and $L_{q}$ is the quark orbital angular momentum contribution to the spin. From the second moment of GPD, $J_{q}$ is given by,

$$
J_{q}=\frac{1}{2} \int_{-1}^{1} d x x\left\{H^{q}(x, \xi, t=0)+E^{q} q(x, \xi, t=0)\right\}
$$

This provides a way to determine the quark angular momentum contribution to the proton spin and potentially solve the "spin-puzzle"

\section{Polynomiality Condition}

From some general properties of Fourier transforms, the $n^{\text {th }} x$ moment of the GPD must be a polynomial of order $n+j$ due to Lorentz invariance. $j=0$ if $\mathrm{n}$ is even and $j=1$ if $\mathrm{n}$ is odd. For H GPD we can write,

$$
\left.\int_{-1}^{1} x^{n} H(x, \xi, t) d x=a_{0}+a_{2} \xi^{2}+a_{4} \xi^{4}+\ldots+a_{(} n+j\right) \xi^{n+j}
$$


Due to time reversal invarience $H(x,-\xi, t)=H(x, \xi, t)$ only the even powers of $\xi$ appear in the polynomial.

\subsection{DEEPLY VIRTUAL COMPTON SCATTERING - ACCESSING GPDS IN EXPERIMENT}

Experimentally we can use $e p \rightarrow e p \gamma$ process at high energy to access GPDs. In this case, we are interested in a virtual compton scattering, where a virtual photon emitted from the incoming electron interacts with a parton picked out from the nucleon in a billiard-ball like collision. The parton momentarily absorbs this virtual photon and then emits a real photon when it returns back to the nucleon, keeping the nucleon intact. Figure 5 shows the handbag diagram of the process in the single photon exchange approximation.

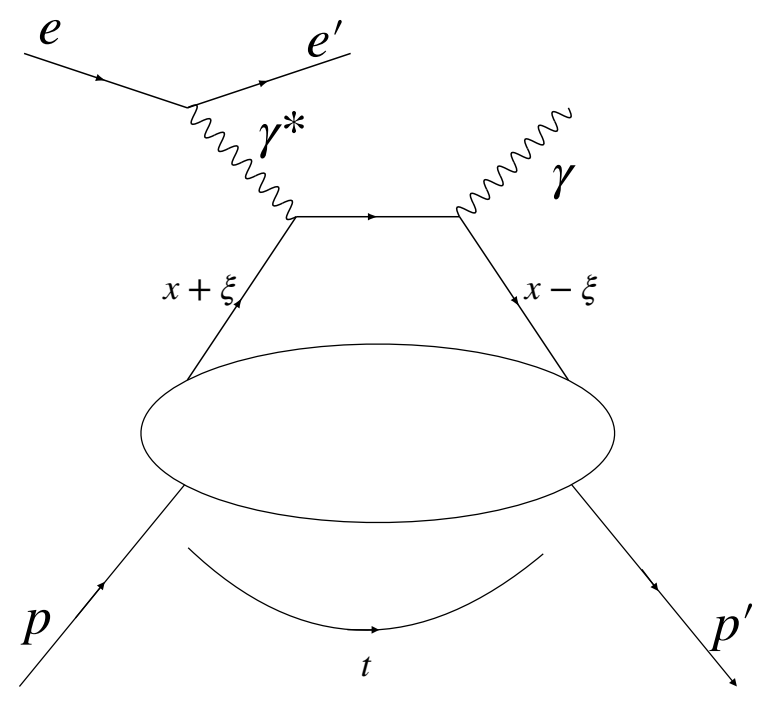

FIG. 5: Figure shows the leading order - twist 2 handbag diagram of underlying virtual compton scattering of the $e p \rightarrow e^{\prime} p^{\prime} \gamma$ process. Virtual photon emitted by the incoming electron interacts with a parton picked out from a nucleon in a billiard-ball like collision. The parton momentarily absorbs this virtual photon and then emits a real photon when it returns back to the nucleon, keeping the nucleon intact. $x+\xi$ is the longitudinal momentum fraction carried by the struck quark and $x-\xi$ is the longitudinal momentum fraction carried by quark after emitting the real photon when returning to the final state. $t=\Delta^{2}=\left(p^{\prime}-p\right)^{2}$ is the total transfer of 4-momentum squared at the proton vertex. $\xi \sim \frac{x_{B}}{2-X_{B}}$ is called the skewness. 


\subsubsection{PARALLEL PROCESSES - EXPERIMENTAL COMPLEXITIES}

In an experiment, liquid hydrogen target is bombarded with accelerated electrons, and many different processes can occur. To identify the DVCS events from all possible reactions of $e-p$ scattering we detect the final state electron and photon and infer the proton from a missing mass cut. The same set of final state can occur in a $e-p$ scattering via a competing QED process called Bethe-Heitler $(\mathrm{BH})$, where the final state photon is emitted from the electron rather than the proton. DVCS and Bethe-Heitler shown in figure 6 are experimentally indistinguishable but the Bethe-Heitler is a well known process and completely calculable within $2 \%$ uncertainty. Moreover the interference of Bethe-Heitler with DVCS, in fact boosts the final cross section and enables us to measure the real and imaginary parts of the DVCS amplitudes as shown in the next section.

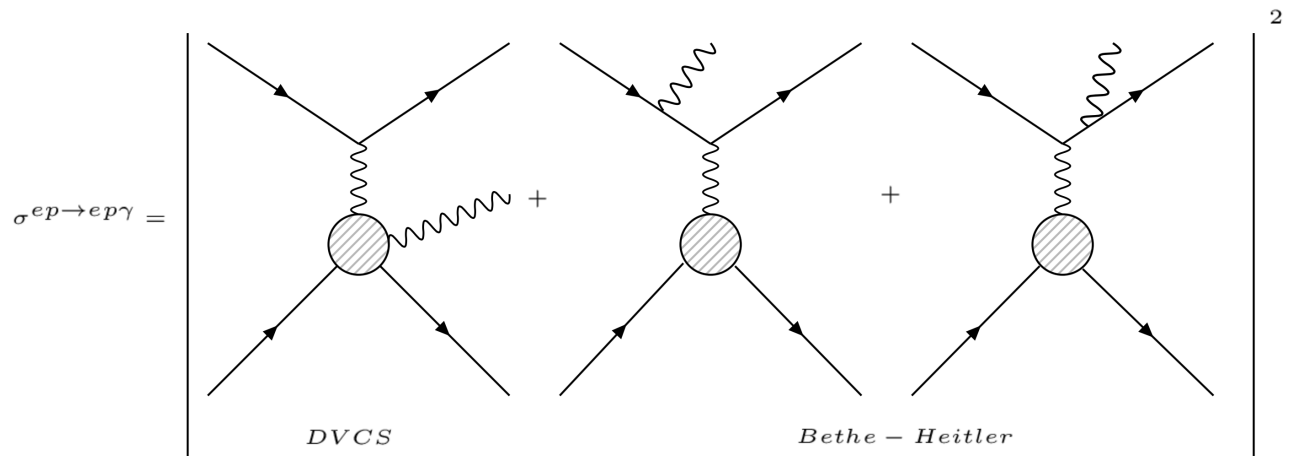

FIG. 6: Figure shows the DVCS process and Bethe-Heitler. In DVCS the final state photon is emitted from the nucleon but in $\mathrm{BH}$ the final state photons are bremsstrahlung radiation from either the incoming or the scattered electron.

A DIS process often called Associated DVCS shown in equation 34 is only separated from the DVCS process by the very small mass of $\pi^{0}$ and leak in to the exclusive $e p \rightarrow e^{\prime} p^{\prime} \gamma$ spectrum after the missing mass cut as a SIDIS background. This results in a systematic uncertainty and discussed in section 6.9 .2

$$
e p \rightarrow e^{\prime} \gamma \Delta^{+} \rightarrow\left\{\begin{array}{l}
e^{\prime}+\gamma+p^{\prime}+\pi^{0} \\
e^{\prime}+\gamma+n+\pi^{+} ; \mathrm{n} \text { is neutron }
\end{array}\right.
$$

As we discussed in section 2.3.1 the hit quark in a hard exclusive process can sometimes radiate a hadronic particle instead of a photon as in DVCS. In the case of DVCS3 experiment 
we have the deeply virtual meson production (DVMP), where the hit quark radiates a $\pi^{0}$. This process in itself interesting and can be used to perform a flavor decomposition of underlying GPDs and hence separately studied in [28, 16]. For the purposes of DVCS, this process can contribute to photon contamination due to the asymetric $\pi^{0}$ decay as discussed in section 6.6.2.

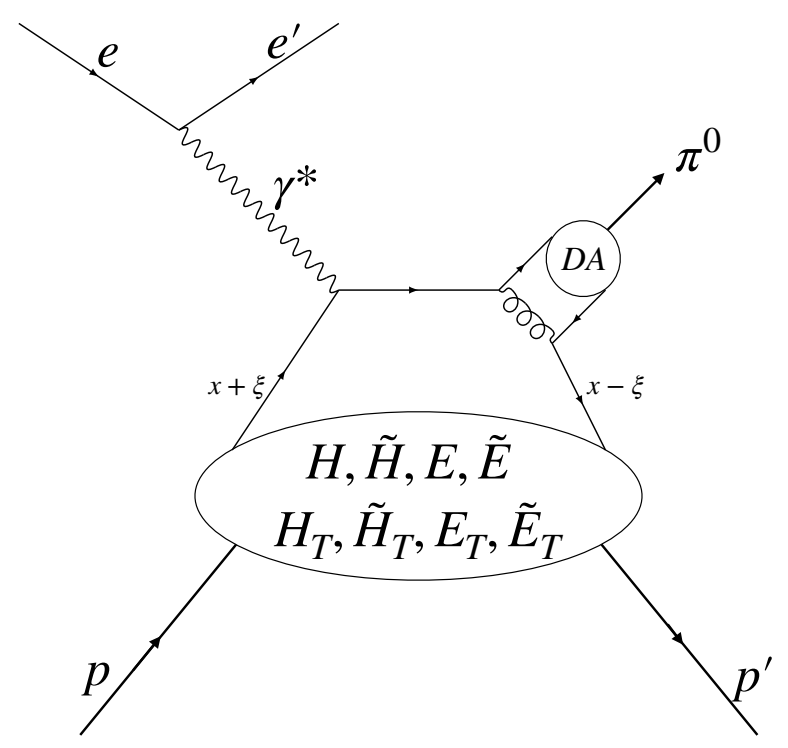

FIG. 7: Figure shows the handbag diagram of Deeply Virtual Meson Production. The process has an additional layer of theoretical complexity via the pion distribution amplitude, DA. The soft part has additional GPDs called transversity GPDs.

\subsubsection{DVCS : BH CROSS SECTION}

Four fold cross section for the $e p \rightarrow e p \gamma$ process is given as follows [12].

$$
\frac{d \sigma}{d x_{B} d Q^{2} d t d \phi}=\frac{\alpha^{3} x_{B} y^{2}}{8 \pi Q^{4} \sqrt{1+\epsilon^{2}}}\left|\frac{\mathcal{T}}{e^{3}}\right|^{2}
$$

here, $\alpha$ is the electro-magnetic coupling constant and $e$ is unit electric charge. $\epsilon=2 x_{B} \frac{M}{Q}$ with $\mathrm{M}$ being the mass of the proton and $y=\frac{p_{1} \cdot q_{1}}{p_{1} \cdot k}$ which will boil down to $\frac{\nu}{k}$ in the lab frame. $\nu=\frac{Q^{2}}{2 M x_{B}}$ and $k$ is the beam energy. $x_{B}, Q^{2}$ and $t$ are Bjorken variable, photon virtuality and four-momentum transfer discussed in previous sections. The angle $\phi$ is of particular interest and discussed bellow.

The total amplitude $\mathcal{T}$ is a superposition of the DVCS and BH amplitudes as discussed in section 2.4.1. The amplitude-squared includes the modulus squared of the DVCS and BH 
amplitudes, and an Interference term between them, given bellow.

$$
\begin{aligned}
\mathcal{T}^{2} & =\left|\mathcal{T}^{\mathcal{B H}}\right|^{2}+\mathcal{T}^{\mathcal{D V C S}}{ }^{2}+\mathcal{I} \\
\mathcal{I} & =\mathcal{T}^{\mathcal{D V C S}}\left(\mathcal{T}^{\mathcal{B H}}\right)^{*}+\left(\mathcal{T}^{\mathcal{D V C S}}\right)^{*} \mathcal{T}^{\mathcal{B H}}
\end{aligned}
$$

\section{Angular Harmonics and Compton Form Factors}

GPDs parametrize the DVCS amplitude, but are not directly accessible in the experiment. They appear via a set of complex integrals called Compton Form Factors (CFFs). A.V. Belitsky, D. Müller and A. Kirchner [14, 12, 13] performed a finite harmonic expansion of the cross section terms of equation 36 in terms of an azimuthal angle $\phi$, as,

$$
\begin{aligned}
\left|\mathcal{T}^{\mathcal{B H}}{ }^{2}\right| & =\frac{e^{6}}{\chi_{B}^{2} y^{2}\left(1+\epsilon^{2}\right)^{2} t \mathcal{P}_{1}(\phi) \mathcal{P}_{2}(\phi)}\left\{c_{0}^{B H}+\sum_{n=1}^{2} c_{n}^{B H} \cos (n \phi)+s_{1}^{B H} \sin (\phi)\right\} \\
\left|\mathcal{T}^{\mathcal{D V} \mathcal{C} \mathcal{S}^{2}}\right| & =\frac{e^{6}}{y^{2} Q^{2}}\left\{c_{0}^{D V C S}+\sum_{n=1}^{2}\left(c_{n}^{D V C S} \cos (n \phi)+\lambda s_{n}^{D V C S} \sin (n \phi)\right)\right\} \\
|\mathcal{I}| & =\frac{e^{6}}{\chi_{B} y^{3} t \mathcal{P}_{1}(\phi) \mathcal{P}_{2}(\phi)}\left\{c_{0}^{\mathcal{I}}+\sum_{n=1}^{3}\left(c_{n}^{\mathcal{I}} \cos (n \phi)+\lambda s_{n}^{\mathcal{I}} \sin (n \phi)\right)\right\} \\
C_{j}^{T} \in\left\{c_{n}^{T}, s_{n}^{T} \mid T\right. & =B H, D V C S, \mathcal{I} ; n=0 \ldots 3\} \text { are called angular harmonics. } C_{j}^{B H} \text { terms }
\end{aligned}
$$
are bilinear combinations of Dirac and Pauli form factors discussed in section 2.1. These terms are exactly calculated by Belitsky et. al in [14]. $C_{j}^{D V C S}$ terms are bilinear combinations of Compton Form Factors. These terms are given in [12]. $C_{j}^{\mathcal{I}}$ terms are linear combination in both elastic form factors and CFFs and are given in [13]. We use parametrization for the Elastic Form Factors given by J. Arrington et al, [9] in our computations.

$\mathcal{P}_{1}(\phi)$ and $\mathcal{P}_{2}(\phi)$ are two lepton propagators, which also introduce angular dependency to the interference term. The angle of expansion $\phi$ is the angle between the leptonic plane formed by $\left\{k, k^{\prime}, q\right\}$ and hadronic plane formed by $\left\{p, p^{\prime}, q^{\prime}\right\}$ shown in figure 8 . Using Trento conventions [10] we can define $\phi$ as,

$$
\begin{aligned}
\cos \phi & =\frac{\hat{q} \times k}{|\hat{q} \times k|} \cdot \frac{\hat{q} \times p^{\prime}}{\left|\hat{q} \times p^{\prime}\right|} \\
\sin \phi & =\frac{\left(k \times p^{\prime}\right) \cdot \hat{q}}{|\hat{q} \times k|\left|\hat{q} \times p^{\prime}\right|} \\
\hat{q} & =\frac{k-k^{\prime}}{|k-k|}
\end{aligned}
$$




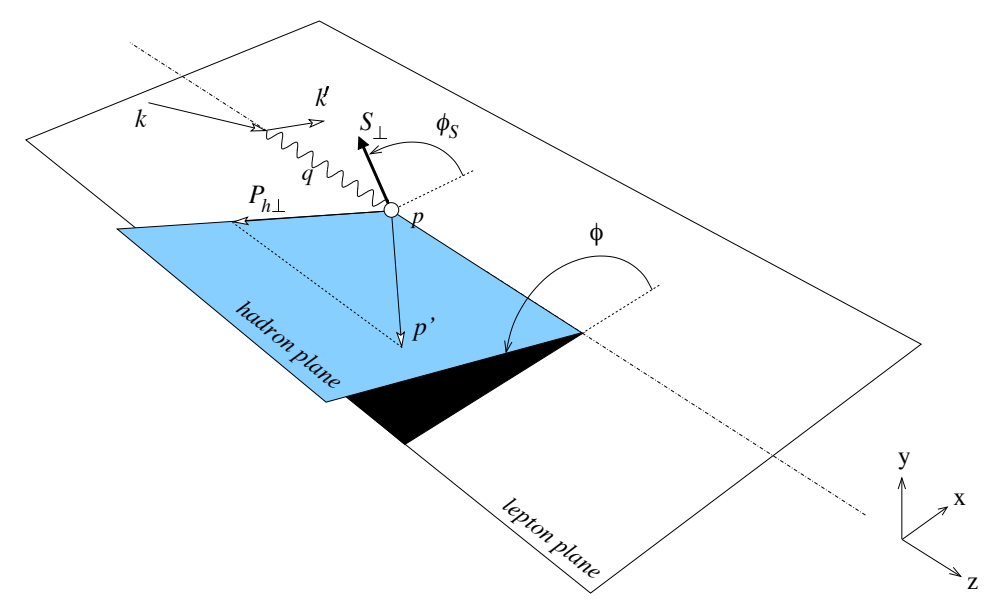

FIG. 8: Figure shows the lepton scattering plane and hadron scattering plane from [10]. $\phi$ defined in eqaution 40, is the azimuthal angle used for harmonic expansion in this text

\subsection{EXPERIMENTAL OVERVIEW OF DVCS AROUND THE WORLD}

As we have seen in this chapter, extraction of GPDs provide many benefits in furthering our understanding of the fundamental nature of universe. The DVCS process has the advantage of conceptual simplicity, but has some experimental challenges. In the last two decades, there were many experiments conducted for the study of GPDs across the world and many are planned well in to the next decades. I will present a very brief overview of some of the past present and future efforts to contextualize this work. [27] presents a detailed account.

\subsubsection{HERA - HADRON ELECTRON RING ACCELERATOR}

HERA was a ring accelerator accelerating both leptons and hadrons at DESY Laboratory in Germany. This was the only lepton - hadron collider operational in its time and powered many collider and fixed target experiments. Collider experiments like ZEUS and H1 utilized up to $27 \mathrm{GeV}$ electron or positron beam and an up to $920 \mathrm{GeV}$ proton beam and able to measure pure DVCS cross sections with minimal Bethe-Heitler interference in the sea quark region of $10^{-4} \leq x_{B} \leq 10^{-2}$ [4]. They were also able to measure the evolution of the DVCS cross section with $Q^{2}, W$ and $t$ [23]. Due to the availability of both electron and positron beam they also measured beam charge asymmetries [1. HERMES is another collaboration at DESY using HERA but they performed fixed target experiments using positron beams 


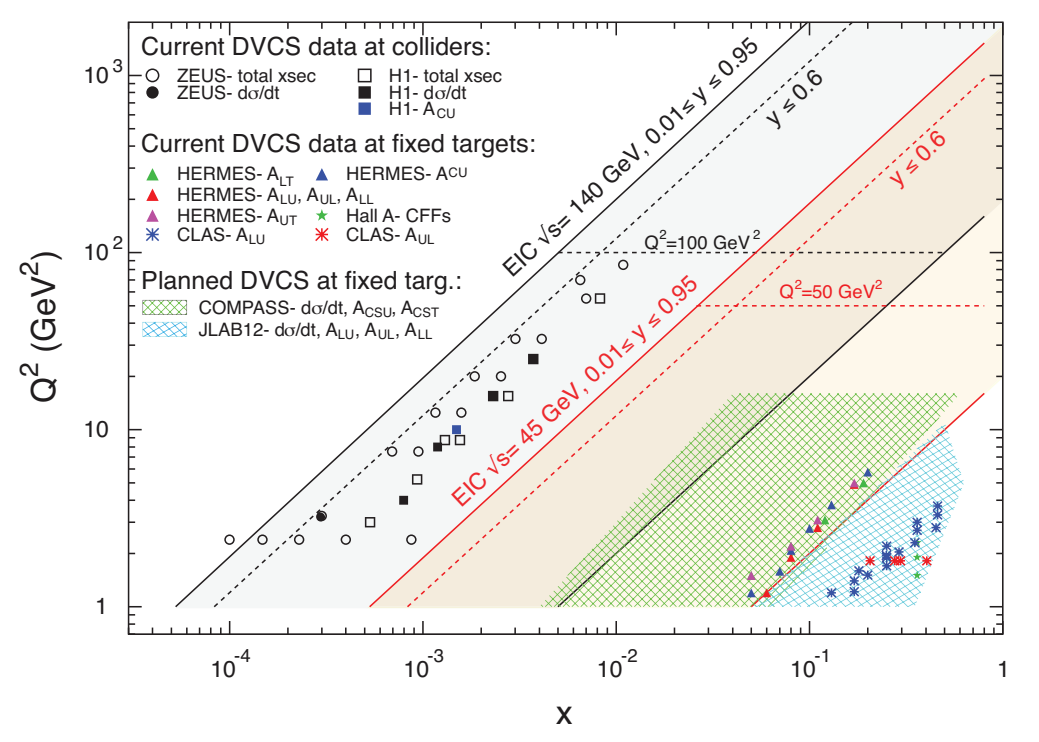

FIG. 9: Figure shows the combined kinematic coverage for GPD studies via DVCS pastpresent-and future [2]. JLab kinematics are mostly in the valence quark region. After HERA, the proposed Electron Ion Collider will provide coverage in to the gluon region.

on gaseous proton target. HERMES measured a full set of asymmetries including the only available DVCS target-spin asymmetry data with transversely polarized target [22]

\subsubsection{CEBAF - JEFFERSON LAB}

Jefferson lab has two complementary DVCS programs in two separate experimental halls. CLAS detector (CEBAF Large Acceptance Spectrometer) provides larger angular acceptance at the expense of luminosity. Hall A and Hall C provides higher luminosity at the expense of acceptance. CLAS collaboration is working on DVCS experiments from 2001 and published azimuthal angle dependency of beam spin asymmetry to kick off the program [77]. In 2004 the very first dedicated DVCS experiment, E00-110 ran in Hall A. It used polarized electron beam on liquid hydrogen target and measured unpolarized and helicity dependent cross sections and performed a scaling test [19]. Another experiment on the same year used a deuterium target and published the first neutron DVCS data [60]. In 2008 second generation experiment at Hall A measured the DVCS coross section for the same kinematics as the 2004 experiment but with two different beam energies to perform a Rosenbluth like separation of $|D V C S|^{2}$ and $\mathcal{I}$ terms. 


\subsubsection{PLANED AND FUTURE EXPERIMENTS}

In Jefferson Lab, E12-06-119 experiment at CLAS12 will expand the existing kinematic range and provide higher statistics for the DVCS on proton. E12-11-003 will expand the neutron DVCS with high accuracy. CLAS collaboration also has plans to measure a complete set of observables akin to the HERMES measurement. Hall C experiment E12-13-010 will expand the Hall A kinematic region as well as provide another set of data at the same kinematics with different beam energies to separate the $|D V C S|^{2}$ and $\mathcal{I}$ terms

\subsection{THE E12-06-114 EXPERIMENT - DVCS3}

E12-06-114, the subject of this thesis is the $3^{\text {rd }}$ generation dedicated DVCS experiment at Hall A of Jefferson Lab. First generation experiments acquired data with $5.75 \mathrm{GeV}$ beam. Second generation experiment used 2 different beam energies for each kinematics, chosen from $3.55 \mathrm{GeV}, 4.55 \mathrm{GeV}$ and $5.55 \mathrm{GeV}$ polarized electron beam. DVCS3 is the first experiment running after the $12 \mathrm{GeV}$ upgrade of Hall A. It ran from Fall 2014 to Fall 2016 in three separate run periods (Fall 2014, Spring 2016 and Fall 2016) and used electron beam of $7-11 \mathrm{GeV} / \mathrm{c}$ and accumulated data in 9 of the originally planed 11 different kinematic settings.

DVCS3 was the first experiment running at JLab after the $12 \mathrm{GeV}$ upgrade and it came with some unique challenges. The status of the Q1 magnet of the High Resolution Spectrometers during the experiment is summarized bellow and discussed in section 4.3.1.

- Spring 2016 : Magnet couldn’t run with full field without quenching.

- 48_1 : 100\% tuned

- 48_2: $62 \%$ tuned

- $48 \_3: 85 \%$ tuned

- 48_4 : 74\% tuned

- Fall 2016 : Replaced magnets, discovered to be saturating at higher current

- 36_2 : 0.8\% saturated

- 36_3 : 6.4\% saturated

- 60_1 : 3.0\% saturated

- 60_3 : 0.7\% saturated 
Table 1 outlines the different kinematic settings, run periods and total accumulated electric charge. Figure 10 Shows the kinematic coverage of the accumulated data.

\begin{tabular}{|c|c|c|c|c|c|}
\hline Run Period & Kin Label & $E_{\text {Beam }}$ & $x_{b}$ & $Q^{2}\left(\mathrm{GeV}^{2}\right)$ & Charge $(C)$ \\
\hline Fall 2014 & $36 \_1$ & 7.383 & 0.36 & 3.20 & 1.8955 \\
\hline Fall 2016 & $36 \_2$ & 8.521 & 0.36 & 3.60 & 1.63791 \\
\hline Fall 2016 & $36 \_3$ & 10.591 & 0.36 & 4.47 & 1.24659 \\
\hline Spring 2016 & $48 \_1$ & 4.487 & 0.48 & 2.7 & 2.17742 \\
\hline Spring 2016 & $48 \_2$ & 8.851 & 0.48 & 4.36 & 2.08707 \\
\hline Spring 2016 & $48 \_3$ & 8.847 & 0.48 & 5.34 & 3.63362 \\
\hline Spring 2016 & $48 \_4$ & 10.992 & 0.48 & 6.90 & 5.66845 \\
\hline Fall 2016 & 60_1 & 8.521 & 0.60 & 5.54 & 5.89503 \\
\hline No Data & 60_2 & - & 0.60 & 6.1 & 0 \\
\hline Fall 2016 & 60_3 & 10.591 & 0.60 & 8.4 & 18.38649 \\
\hline No Data & $60 \_4$ & - & 0.60 & 9.0 & 0 \\
\hline
\end{tabular}

TABLE 1: DVCS3 runs were taken in 9 different kinematic settings, in 3 different run periods spanning 2 years. The experiment used multiple beam energies up to $11 \mathrm{GeV}$. The total acquired charge per kinematic listed here are adjusted to the DAQ deadtime 


\section{DVCS3 Kinematic_Coverage}

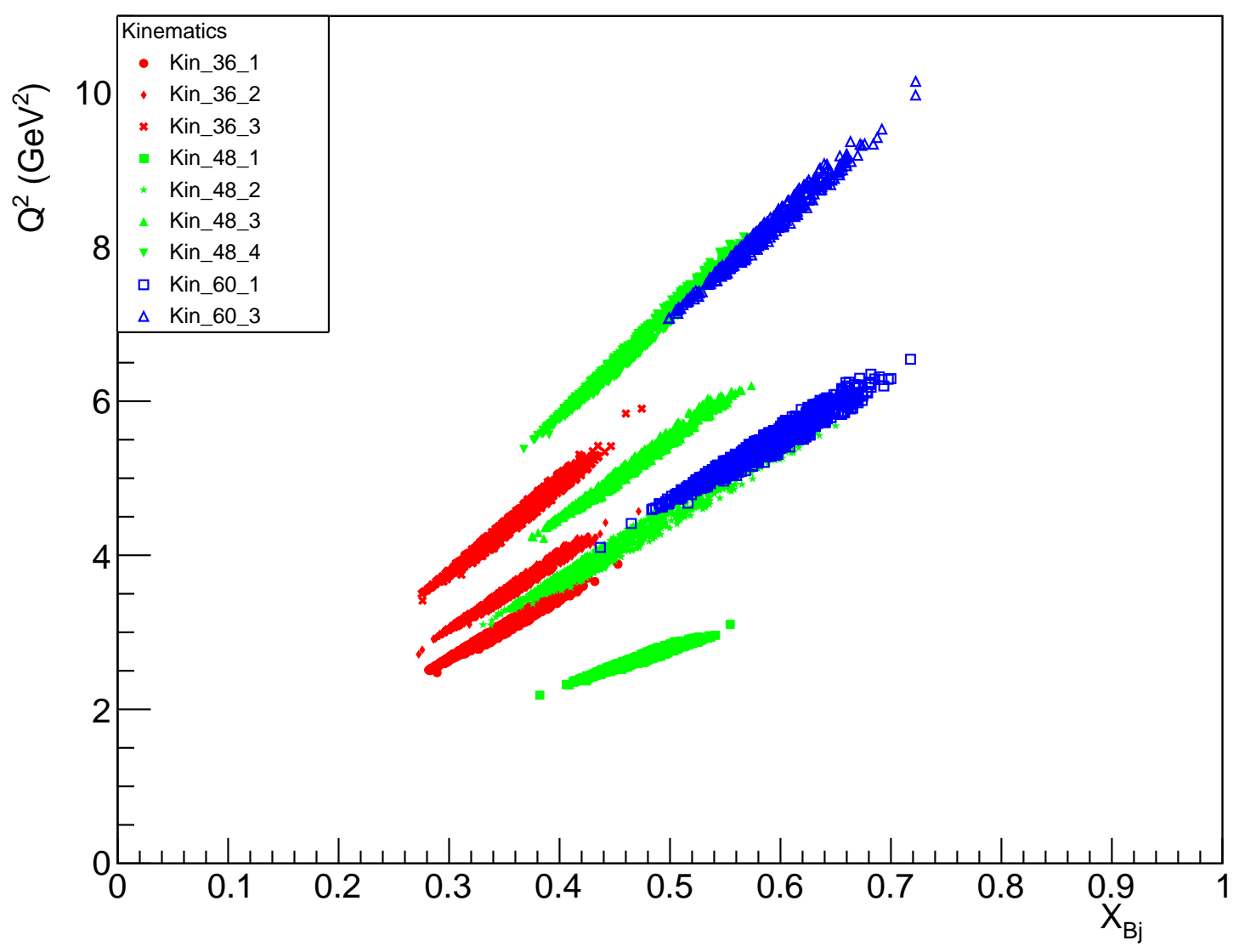

FIG. 10: Figure shows the combined kinematic coverage for DVCS3 experiment from real acquired data. each scatters are roughly 2000 points. Blue is $x_{B}=0.6$, Green is $x_{B}=0.48$ and Red is is $x_{B}=0.36$. 


\section{CHAPTER 3}

\section{EXPERIMENT SETUP AND INSTRUMENTATION}

E12-06-114 (DVCS3) experiment is the $3^{\text {rd }}$ generation experiment of the Thomas Jefferson National Accelerator Facility, Experimental Hall A DVCS Program. It is an electron proton collision experiment, where a cryogentic liquid hydrogen target held inside a $15 \mathrm{~cm}$ long Aluminum cell was bombarded with electrons of energy 6.6 to $11.0 \mathrm{GeV}$. At the heart of the DVCS process is Virtual Compton Scattering, where a virtual photon scatters off of a parton inside the proton in a billiard ball like collision. The virtual photon is emitted from the incoming electron when it comes in close proximity to the proton and the scattered electron is detected in the left high resolution spectrometer (LHRS). If the produced virtual photon is of high enough energy, It can see inside the hadron, to collide with it's constituents. DVCS is an elastic process, wherein the initial proton comes out intact in the final state, which is achieved by the emission of a real photon. The emitted real photon is then detected in a custom electro-magnetic calorimeter constructed of $208 \mathrm{PbF}_{2}$ crystals. The goal of the experiment was to verify the scaling behavior observed in the previous experiments, with measurements over a broader kinematic range. Subsequent sections of this chapter describes the Instrumentation in more detail.

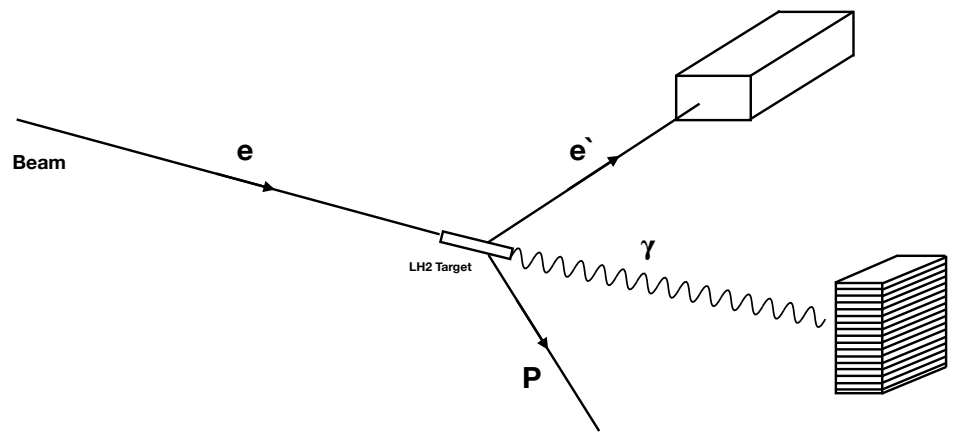

FIG. 11: A cartoon diagram of the DVCS3 experiment setup in Jefferson Lab, Hall A. Scattered electrons are detected in the Left High Resolution Spectrometer and photons are detected in DVCS $\mathrm{PbF}_{2}$ Calorimeter. 


\subsection{THOMAS JEFFERSON NATIONAL ACCELERATOR FACILITY}

Thomas Jefferson National Accelerator Facility (JLab) in Newport News, Virginia, is an electron beam accelerator facility of unique stature. The Continuous Electron Beam Accelerator Facility (CEBAF, Figure 12) accelerates electrons up to $12 \mathrm{GeV}$ (24,000 times the rest mass) with effectively $100 \%$ duty factor, meaning the beam is continuous on the scale of the integration time of the particle detectors. The electron beam is a continuous train of short $100 \mathrm{ps}$ pulses recurring at $1497 \mathrm{MHz}$. the delivery of electron pulses to to the Halls occur at $249.5 \mathrm{MHz}$ during 4 hall operation or $499 \mathrm{MHz}$ during 3 hall operations [34. The high duty factor is achieved by the use of superconducting radio- frequency cavities (SRF). CEBAF can currently provide beam to 4 experimental Halls each with its own unique set of advantages, simultaneously up to a total of $200 \mu \mathrm{A}$ current. CEBAF consists of two linear accelerators with 25 cryomodules each connected via arcs of bending magnets. Each linac provides $1.1 \mathrm{GeV}$ of energy per pass. Electron bunches from the injector make up to 5 passes through the accelerator before directed to experimental Halls A, B or C making them capable of receiving up to $11 \mathrm{GeV}$ electron beam. Electron bunches can make one additional pass through the north linac on it's way to the newly established Hall D making it capable of receiving up to $12 \mathrm{GeV}$ beam.

The beam to the accelerators are injected from a GaAs photo cathode gun with an acceleration of $45 \mathrm{MeV}$, using the first set of RF cavities. The gun is illuminated by 4, 850 $\mathrm{nm}$ lasers each having a phase offset with respect to one another, operating at $250 \mathrm{MHz}$ to pump electrons to 4 different halls. At the end of full acceleration, an RF separator is used to separate the beam delivered to each experiment hall, using the phase offset of the laser. The lasers are circularly polarized using a Pockels cell [66], whose voltages are varied to flip the helicity of the beam at a frequency of $30 \mathrm{~Hz}$.

\subsection{HALL-A}

Hall A can be operated at high luminosity, of the order of $10^{38} \mathrm{~cm}^{-2} \mathrm{~s}^{-1}$ and hence suitable for experiments such as DVCS. Experimental Hall A provides two High Resolution Spectrometers with independent moving arms capable of resolving the momenta of detected charged particles to a relative precision of $2 \times 10^{-4}$, within the roughly $6 \mathrm{msr}$ acceptance. The HRS Magnetic optics have the design of QQDQ. The first two quadrupole magnets focus the accepted particles in perpendicular directions to each other. The resolving magnet is a large $6.6 \mathrm{~m}$ long dipole and a $3^{\text {rd }}$ quadrupole magnets sits after the dipole magnet to focus 


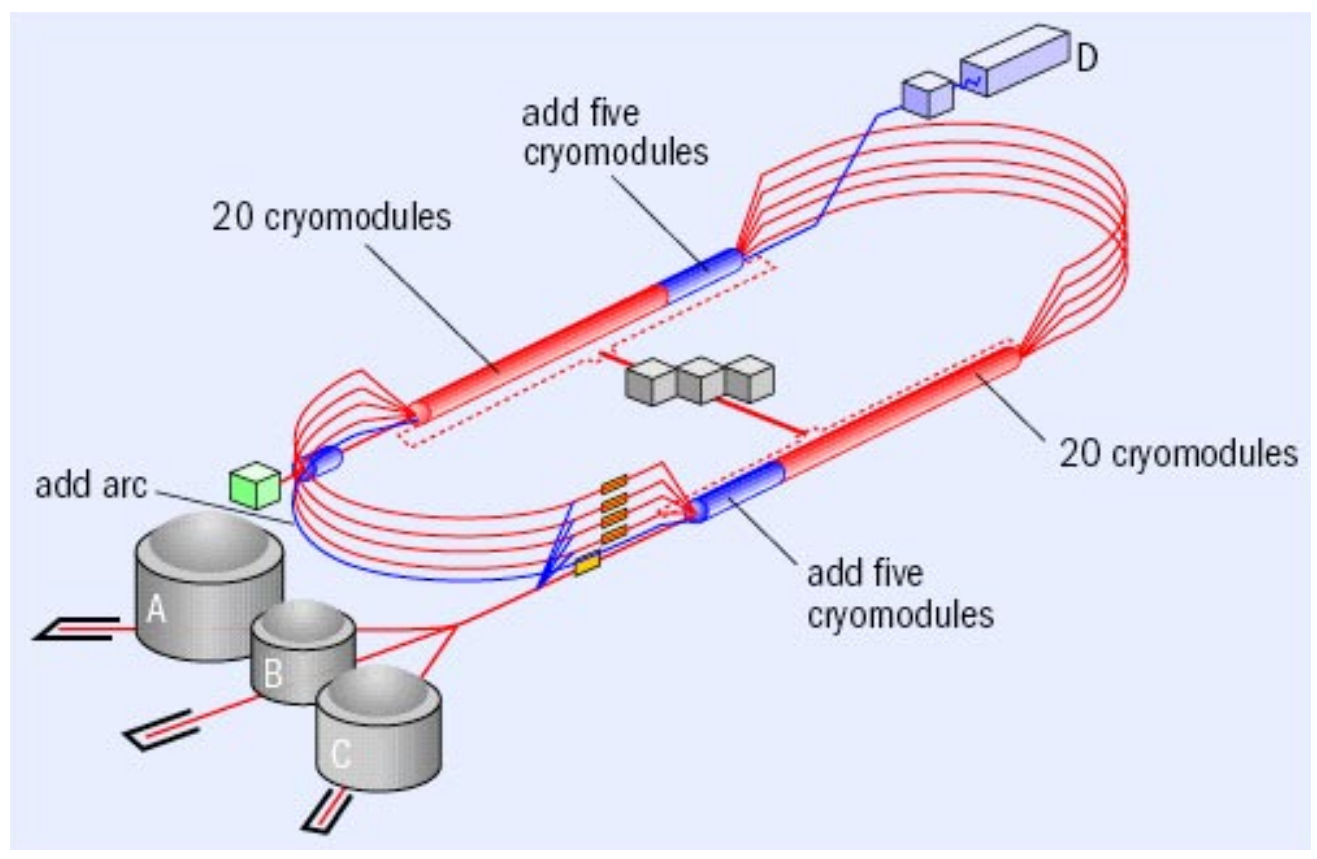

FIG. 12: A schematic diagram of the Continuous Electron Beam Accelerator Facility (CEBAF) of Jefferson Lab. The diagram shows the two linacs and the positions of the 4 experimental halls. Electrons are accelerated up to $45 \mathrm{MeV}$ in the injector, before injection into the north linac. 
the resolved beam in perpendicular direction to the dispersive direction before entering the detector stack. The DVCS3 experiment used the Left arm of the HRS (LHRS) to detect the scattered electron. LHRS has a momentum acceptance of $\pm 4.5 \%$ and angular acceptance of $\pm 30 \mathrm{mr}$ in the horizontal direction and $\pm 60 \mathrm{mr}$ in the vertical direction. A schematic diagram of the Hall is given in Figure 13

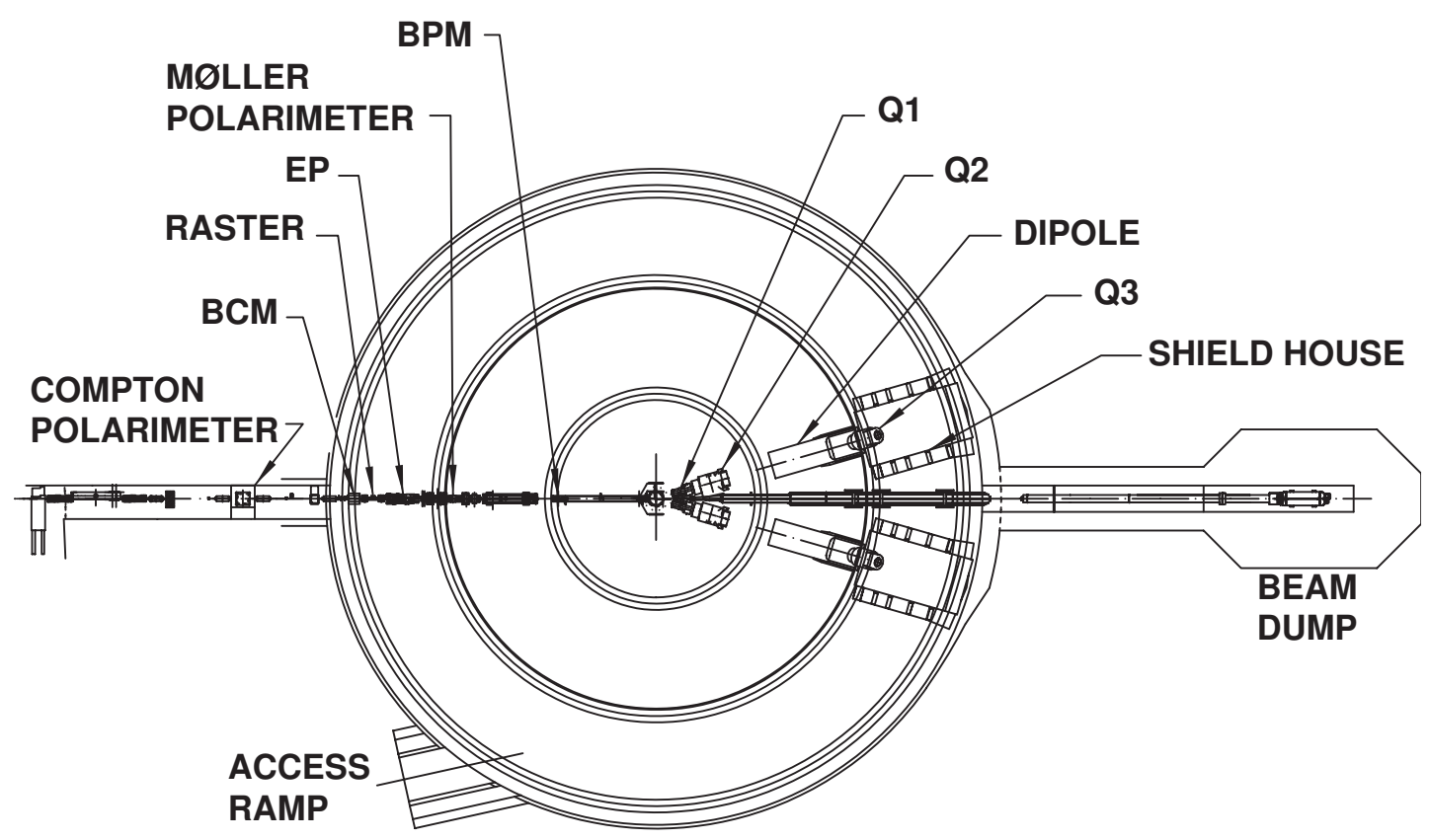

FIG. 13: Diagram of the experimental Hall A setup. Target chamber is in the center.

\subsection{DVCS INSTRUMENTATION}

DVCS uses the standard Hall A instrumentation, together with a custom calorimeter and data acquisition system for gamma-ray detection in coincidence with the scattered electron.

\subsubsection{BEAMLINE}

Hall A beam line consists of several components for the steering and focusing of the beam as well as to measure the necessary properties. 


\section{Polarimetry}

Two polarimeters are available for beam polarization measurements in the hall. The Compton polarimeter provides a non-invasive measurement of the longitudinal beam polarization by measuring the asymmetry of the Compton scattering cross section of the incoming electron beam with a circularly polarized photon beam. Since the measurement is non-invasive, the Compton polarimeter is primarily used to take the beam polarization measurement during an experiment. During the DVCS3 experiment however the Compton polarimeter was not available for part of the data taking due to poor laser alignment issues [28]. The Moller polarimeter was the only option for polarization measurement during those periods. The Moller polarimeter provides an invasive measurement of the beam polarization by measuring the cross section of Moller scattering of the incoming electron beam with polarized atomic electrons in a ferromagnetic foil. This measurement must be performed at lower beam currents and is generally used to cross-check the reading from the Compton polarimeter.

\section{Beam Position Monitors}

A set of wire scanners called Harps are installed throughout the accelerator and experimental halls and operated by the Machine Control Center to measure the absolute beam positions. The scanners contain 3 wires whose positions are known within $200 \mu \mathrm{m}$ and provide a signal when scanned across the the electron beam by a precision stepping motor. The absolute beam position can be measured by inserting the Harp scanner in the path of the beam and moving the wires transversely to observe the current profile [41]. This method is invasive and cannot be used to monitor the beam position while taking experimental data. For that purpose, Hall A provides a pair of devices simply called Beam Position Monitors (BPMs) situated upstream of the target (see Figure 13). BPMs are made of two sets of antennas ( 4 wires in total arranged in the corners of a right cuboid with square cross section) with each wire of the same set placed parallel to the beam and on diametrically opposite sides of the beam pipe. A relative position of the beam can be obtained by comparing the the currents induced in the antennas at the bunch frequency. Calibrating the BPM against the Harp and verifying it against the carbon hole target gives the absolute position of the beam. 


\section{Beam Current Monitors}

Hall A provides a system of an Unser monitor and two RF cavities for Beam Current Monitoring [5]. The Unser monitor is a transformer which provides a nominal output of $\frac{4 m V}{\mu A}$. The Unser is calibrated by sending a known current through a wire passing through the monitor. Due to the drift of Unser motor's output signal over time, it is not used for continuous current monitoring. Two resonant RF cavities produce voltage outputs proportional to the beam current. The output signal from each cavity are split in two and sent for sampling or integration. For the integrated output, the signal down converted from the frequency of the beam bunches to approximately $30 \mathrm{kHz}$ and then sent through an RMS to DC converter. This quasi-DC signal is then sent to a voltage to frequency converter, the pulse train of which is sent to a scaler. This scaler simply accumulate during the run and hence represent the time integrated voltage level, and hence the accumulated charge of the beam. The original RMS-DC signal is linear for current from $5 \mu A$ to over $200 \mu A$. To extend the non linear region, two amplifiers with gain factors 3 and 10 are introduced, making total of 6 signal output from two cavities for a single spectrometer arm.

\section{Raster}

The DVCS Experiment used very high intensity electron beams which, combined with the small transverse cross section could locally heat the liquid hydrogen target and result in density variation as well as damaging the target system. A raster system comprised of two electro-magnets driven at incommensurate frequencies around at $25 \mathrm{kHz}$. This spreads the beam uniformly to dissipate the heat on the target. DVCS3 experiment used a $2 \mathrm{~mm} \times 2 \mathrm{~mm}$ square raster at the target. the instantaneous driving current of the raster system together with the BPM signal are recorded event-by-event and used to reconstruct the transverse vertex position in the target of the scattered electron.

\section{Beam Energy Measurement}

Beam energy for the DVCS3 experiment was measured by utilizing the Arc method or most commonly known as Arc energy measurement. The $40 \mathrm{~m}$ arc section between Hall-A and the accelerator contains 8 dipoles under vacuum which deflects the electron beam. The bending angle of the electron beam through the arc is measured using wire scanners at the entry and exit points of the arc. The field integrals of the dipoles are measured using an identical dipole situated in the next room to the arc dipoles and driven 
in series with them. the momentum of the electron beam is then given by equation 41 with $k=0.299792 \mathrm{GeVrad} T^{-1} m^{-1} c^{-1}[42]$.

$$
p=k \frac{\int \vec{B} \wedge \overrightarrow{d l}}{\theta}
$$

\section{Target System}

Hall-A provided a target ladder assembly with multiple targets mounted. This ladder can be controlled remotely and moved vertically to place a specific target in the beam line according to the experimental requirement. The target ladder is mounted inside a cylindrical vacuum chamber of $1143 \mathrm{~mm}$ diameter and contains experimental targets as well as control targets.

- Cryogenic Target: Hall-A DVCS target system had 3 cells cooled using circulating liquid helium. These cells house the appropriate cryogenic target, in this case hydrogen. Maximum cooling power available for the target cooling system was $1 \mathrm{~kW}$, which could comfortably accommodate up to $130 \mu \mathrm{A}$ beam current accounting for the $700 \mathrm{~W}$ heat output from the beam on top of the heat output from the fans and other heat pumps. DVCS3 experiment used a $63.5 \mathrm{~mm}$ diameter cylindrical aluminum cell of $15 \mathrm{~cm}$ length as the target housing for Liquid hydrogen target. the operating temperature of the target was $19 \mathrm{~K}$ the pressure was $0.17 \mathrm{MPa}$. At this temperature and pressure the target density was about $0.0723 \mathrm{gcm}^{-3}$

- Optics Target: An assembly of five $1 \mathrm{~mm}$ thick carbon foils spaced $3.75 \mathrm{~cm}$ from each other used primarily to calibrate the magnetic optics of the spectrometer by optimizing the transport matrix. Additional four foils were added to the target during the Spring and Fall 2016 Runs to improve the calibration output.

- Dummy Target: Target assembly also provides a set of empty aluminum cells identical to that housing the cryogenic target to study the scattering background from the aluminum walls of the target housing.

- BeO Target: BeO illuminates when hit by electron beam and a camera can capture this directly and function as direct visualization of the beam and used to center the beam in the target.

- Carbon Hole Target: A thick carbon foil with a $2 \mathrm{~mm}$ diameter hole at the center. This target can be used for beam positioning studies. 
- Empty Target: A position in the target ladder without any target. This is used to minimize the radiation effects when performing invasive beam studies or any other studies which does not require a specific target.

\subsubsection{HIGH RESOLUTION SPECTROMETER DETECTOR ASSEMBLY}

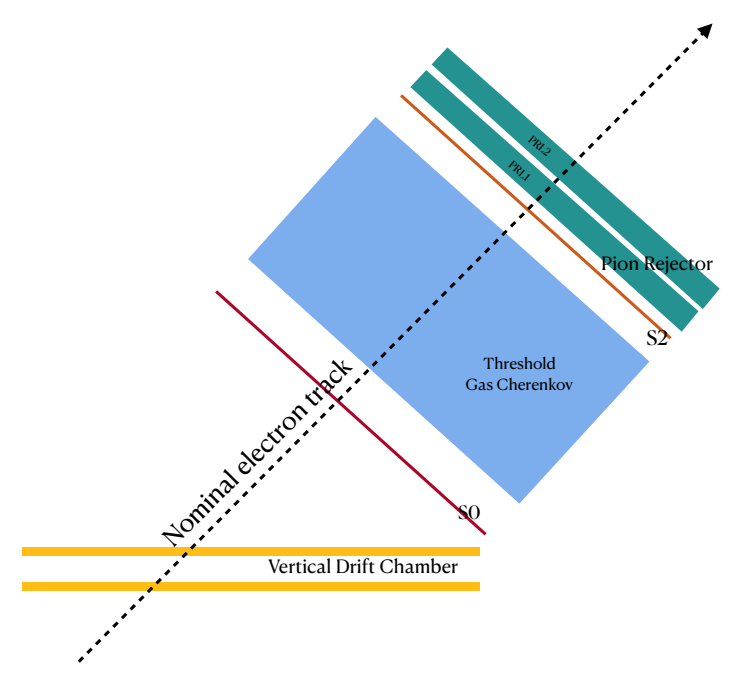

FIG. 14: Cartoon diagrams of the LHRS detector stack showing the various particle and trigger detectors

LHRS detector stack is comprised of a Vertical Drift Chamber (VDC) for tracking, a PbGlass Calorimeter (Pion Rejector - PR) and a Gas Cherenkov detector (CER) for particle identification. Coincidences between $\mathrm{S} 2$, an array of sixteen $5 \mathrm{~mm}$ thin scintillator paddles attach to phototubes on either sides and Gas Cerenkov with the DVCS Calorimeter forms the main trigger. Another large $10 \mathrm{~mm}$ thick single scintillator, called S0 covering the whole vertical length of the detectors paired with two phototubes on top and bottom can be used with either S2 or Gas Cherenkov to form additional triggers. Figure 14 shows a schematic diagram of the detector stack in LHRS.

\section{Pion Rejector}

Hall A detector package contains a pair of $\mathrm{Pb}$-Glass calorimeter each made up of $34 \mathrm{~Pb}$ Glass blocks of $14.5 \times 14.5 \times 30(\mathrm{~cm})$, arranged in $2 \times 17$ array. Figure 15 shows a diagram of Pion Rejector. Each layer of the calorimeter are off-set by $50 \mathrm{~mm}$ and aligned perpendicular 
to the nominal track [5]. The smaller radiation length would ensure only electronic shower will deposit energy while a pion (hadron) is a Minimum Ionizing Particle (MIP).
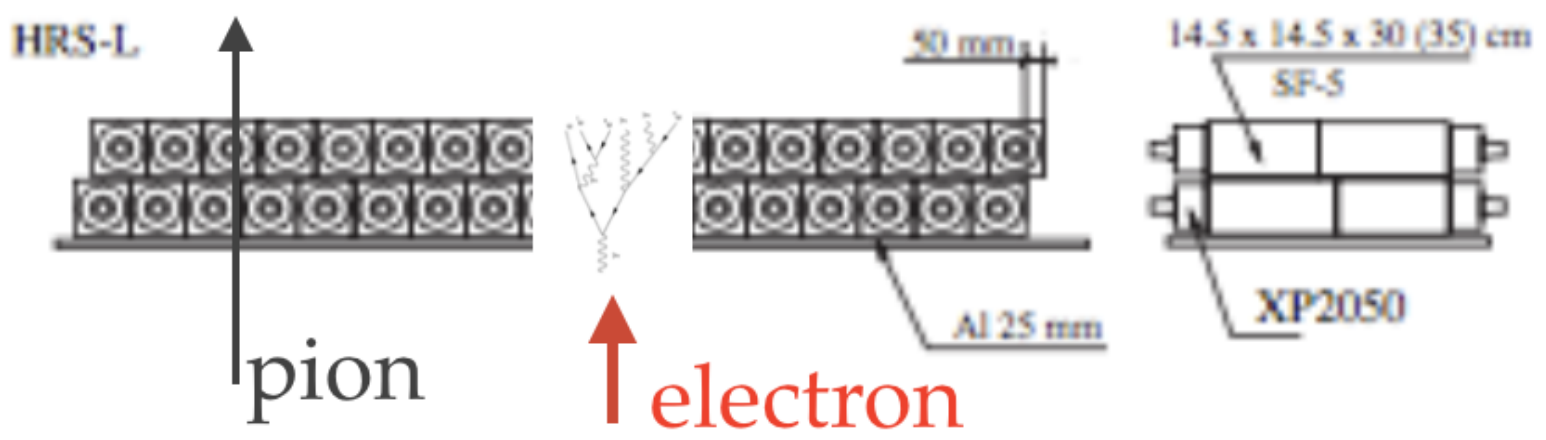

FIG. 15: Hall A LHRS Pion Rejector has 2 identical layers of 34 Pb-Glass blocks.

\subsubsection{GAS CHERENKOV}
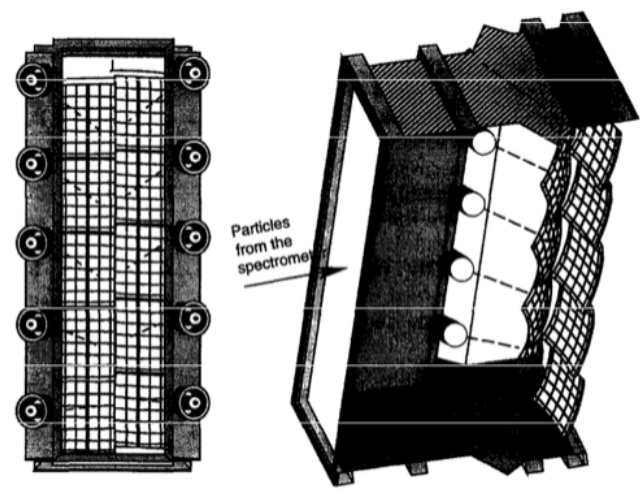

FIG. 16: Gas Cherenkov in Hall A DVCS is a threshold detector with $1.2 \mathrm{~m}$ long chamber filled with atmospheric pressure $\mathrm{CO}_{2}$ that could distinguish between electron and hadron

Figure 16 shows a diagram of the threshold Gas Cherenkov filled with atmospheric pressure $\mathrm{CO}_{2}$ gas. It has 10 spherical mirrors, each viewed by a photo multiplier tube to collect the Cherenkov light generated by the charged particle. The detector has an electron identification efficiency of $99 \%$ with threshold for electrons at $17 \mathrm{MeV} / \mathrm{c}$. Threshold for pions in this setting is $4.8 \mathrm{GeV} / \mathrm{c}$ [5, 46] and above the $4 \mathrm{GeV}$ maximum momentum limit of LHRS. 


\section{Vertical Drift Chamber}

Vertical Drift Chamber (VDC) in Hall A provides the tracking information. It has two chambers (Top and Bottom) each having one set of perpendicular wire planes (U and V) with 368 sense wires [5, 32]. Figure 17 shows the configuration of the VDC in Hall-A. First 16 wires of each end of a wire plane are grounded for field shaping, making the total of 400 wires in each plane 32]. Hall-A Analyzer package runs an algorithm optimized for single cluster scenario to reconstruct the tracks from the raw cluster data. The algorithms performance and shortcomings are discussed in section 4.4.1.
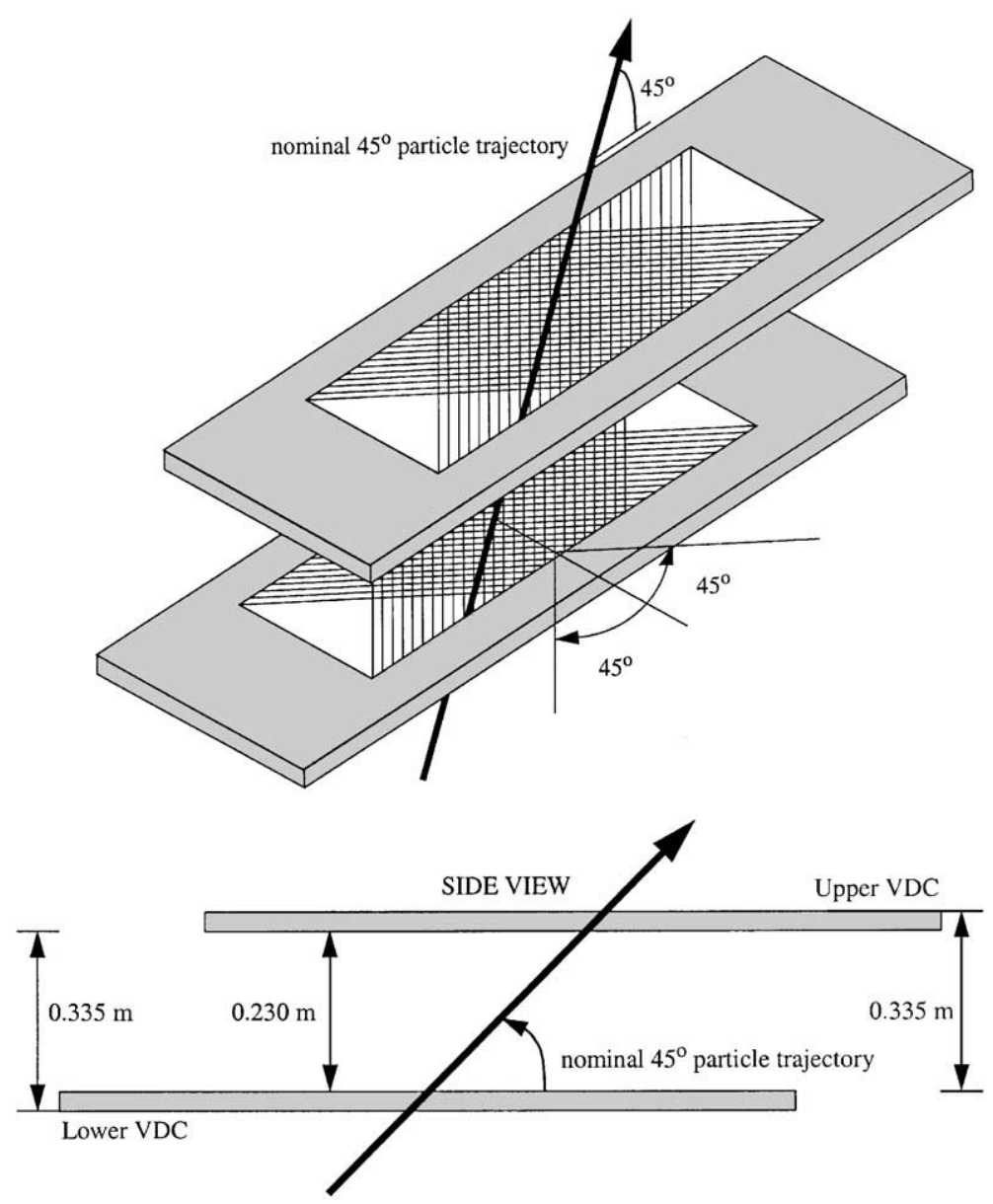

FIG. 17: Side and top view of the Hall A vertical Drift Chamber. VDC has 2 chambers (Top and Bottom) at $335 \mathrm{~mm}$ vertical distance between each other with 2 perpendicular wire planes ( $\mathrm{U}$ and $\mathrm{V}$ ) each having 368 sense wires per plane with $4.24 \mathrm{~mm}$ wire spacing. It sits at a 45 degree angle to the nominal particle trajectory with bottom chamber at $Z_{\text {focal plane }}=0$ and uses standard Argon/Ethane (62\%-38\% by weight) gas mixture. 


\subsection{DVCS ELECTROMAGNETIC CALORIMETER}

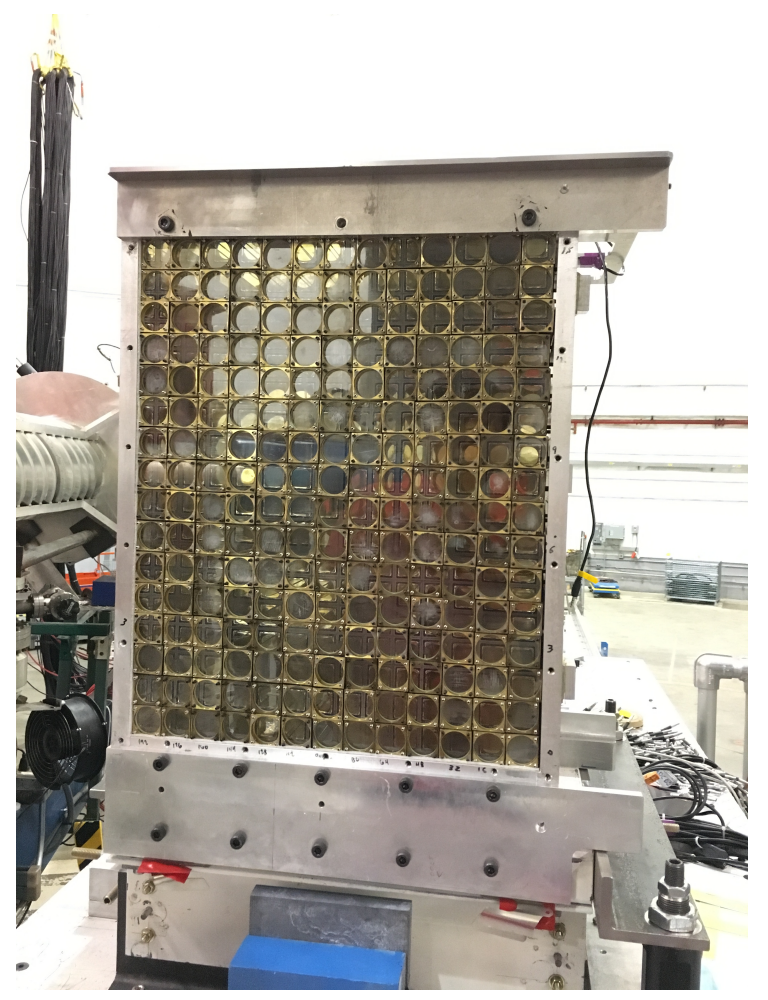

(a) Front of the calorimeter.

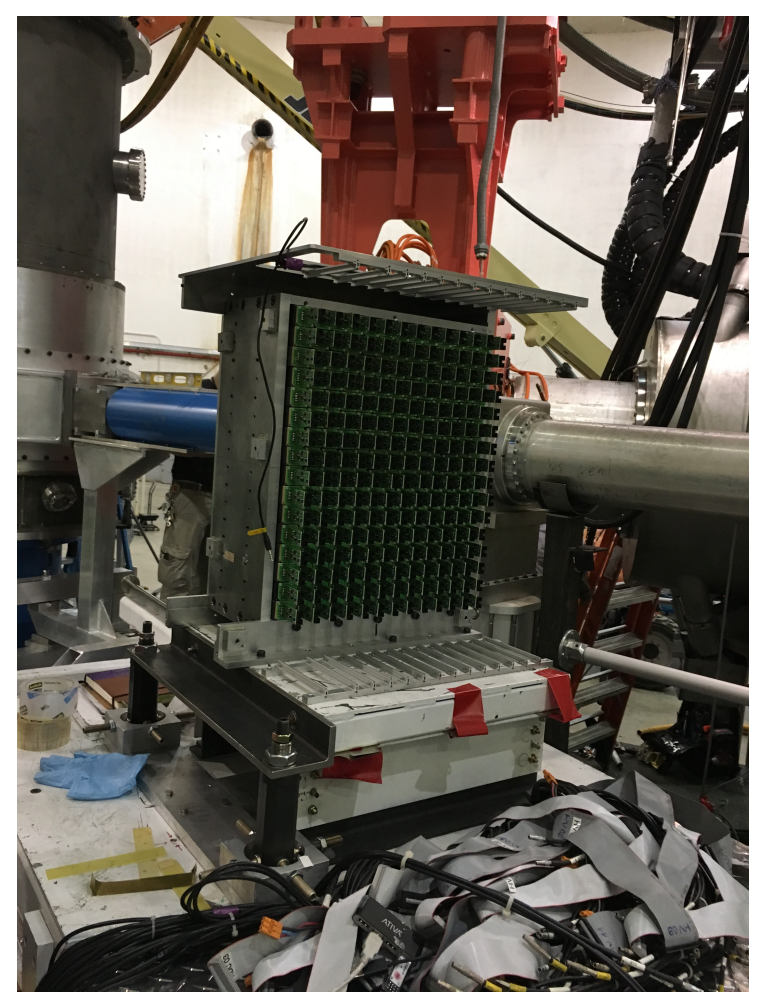

(b) Back of the calorimeter.

FIG. 18: Figure shows the assembled DVCS calorimeter before installing the front and back panels and the black box.

DVCS3 experiment uses a $\mathrm{PbF}_{2}$ calorimeter built from $208 \mathrm{PbF}_{2}$ crystals of $3 \mathrm{~cm} \times$ $3 \mathrm{~cm} \times 18.6 \mathrm{~cm}$ arranged in a $13 \times 16$ array as the photon detector shown in Figure 18. Calorimeter blocks are warped in Tyvek ${ }^{\circledR}$ and then in Tedlar ${ }^{\circledR}$ to prevent light leaks between blocks. This calorimeter is placed between the beam line and LHRS roughly $1 \mathrm{~m}$ to $2.5 \mathrm{~m}$ away from the target depending on the kinematic setting of the experiment. $\mathrm{PbF}_{2}$ is a radiation hard Cherenkov medium with smaller Molière radius. This will also help in resolving the energy of the detected photon with higher accuracy as well as separating the showers from $\pi^{0}$ decay since $90 \%$ of the energy from a photon is deposited in the central block at the same time the block being insensitive to low energy particles. The close proximity of the calorimeter to the beam line would mean higher possibilities of pille-up. This can be dealt with by using capacitively coupled fast response PMTs read by $1 \mathrm{GHz}$ Analogue Ring Sampler (ARS) Digitizers [69]. Additional shielding was installed including a tungsten nose 
shielding to minimize the radiation background from multiple scattering in the scattering chamber reaching the calorimeter, specially the first column closer to the beam pipe.

\subsection{DATA ACQUISITION (DAQ)}

In general, standard Hall-A DAQ system starts with feeding the signal from the endpoint photo multiplier tubes of a detector to Analogue to Digital Converter (ADC), Time to Digital Converters (TDC) and/or scalars in a VME crate. These VME crates are read by Readout Controllers (ROCs) connected to the Trigger supervisor based on the arrival of a trigger. The data collected from by the ROCs are sent to an event builder to build the event information from all pieces of data gathered from various ROCs and passed to the Event Recorder for recording to the local disk which then transferred to the mass storage tape silo. A new trigger is only accepted if all the ROCs are available, if any one of the ROC is busy, the Trigger Supervisor will get a VETO and the event is dropped resulting in an acquisition dead time. Front end of the DAQ is the user controlled piece of software called CODA (CEBAF Online Data Acquisition), which is a tool kit software component to build and customize the DAQ system.

DVCS experiment requires additional pieces of DAQ electronics to manage the high event rate of the DVCS calorimeter due to the high luminosity of the experiment combined with the close proximity of the calorimeter to the beam pipe.

\subsubsection{ANALOGUE RING SAMPLER (ARS) AND DVCS TRIGGER SYSTEM}

As mentioned in section 3.4 each block of the calorimeter is connected to an ARS and sampled at $1 \mathrm{GHz}$. An ARS is made of 128 circular capacitor [30] and the data is moved to the next capacitor in the array every next nano sec resulting in a 128 ns data storage at any given time. When a trigger is received, the ARS data sampling is stopped and the capacitor contents are digitized. Due to large amount of data to be digitized, this results in a $128 \mu s$ dead-time. DVCS experiment employs a 2 level trigger system to minimize this dead time.

DVCS events are triggered by the coincidence between the scintillator array $\mathrm{S} 2$ and the Gas Cherenkov to verify an electron in the spectrometer. When this coincidence is formed, trigger supervisor then looks for a coincidence between the spectrometer and the calorimeter to see for a photon in coincidence with the electron. To establish this coincidence, trigger supervisor looks for the copy of the ARS information send to a fast-ADC. A stop signal is sent to the ARS upon the arrival of the spectrometer trigger and the integrated fast-ADC data is read and summed in any possible $2 \times 2$ neighbors of the blocks to look for a signal 
higher than a set threshold. If such a signal is present an ARS valid is sent and the data is digitized. If the coincidence trigger is not validated, ARS is cleared and resumes sampling. this would reduce the dead time to $500 \mathrm{~ns}$ when the ARS is not validated from the otherwise $128 \mu \mathrm{s}$. 


\section{CHAPTER 4}

\section{HRS DETECTOR CALIBRATION AND EFFICIENCY}

\section{ANALYSIS}

As discussed in section 1.3 the first step in the DVCS cross section analysis process is to identify events with a good electron track in the Left - High Resolution Spectrometer. The DVCS3 experiment ran in parallel with the GMp experiment, which performed a precision measurement of the Proton Elastic Cross Section at high $Q^{2}$ using the Right-HRS in order to extract the proton magnetic form factor $\left(G_{M p}\right)$. GMp group performed the initial calibration of many HRS detectors of both HRS-right and HRS-left. This chapter verifies the performance of the initial calibration and discusses the improvement made when it was necessary and presents the efficiency analysis of each detectors.

\subsection{PION REJECTOR CALIBRATION}

A Pb-Glass calorimeter, called the Pion Rejector described in section 3.3.2, was used to eliminate pions from the detected data. Pion rejector is initially calibrated by extracting a set of coefficients $\left\{C_{i} \mid i=1, \ldots M\right\}$ which translate raw ADC amplitude of each individual $\mathrm{Pb}$ Glass block into a corresponding energy value. These coeficients are obtained by minimizing the following statistics:

$$
\chi^{2}=\sum_{n=1}^{N}\left\{\left[\sum_{i \in M} C_{i}\left(A_{i}^{n}-P_{i}\right)\right]-E_{e}^{n}\right\}^{2}
$$

Here,

$\mathrm{n}$ - Number of given calibration event

i - Number of block in the cluster in the $n^{\text {th }}$ event

$\mathrm{N}$ - Total number of selected calibration events

$\mathrm{M}$ - Total number of $\mathrm{Pb}-$ Glass blocks in the detected cluster of the $n^{\text {th }}$ event

$A_{i}^{n}$ - Amplitude value in the $i^{\text {th }}$ block of the $n^{\text {th }}$ event

$P_{i}$ - Mean pedestal value of the $i^{\text {th }}$ block

$E_{e}^{n}$ - Electron momentum of the $n^{t h}$ calibration event corrected for the extended target effect 
Pion Rejector Amplitudes : PRL1 (Blue), PRL2 (Red), $\chi_{\mathrm{b}}=0.48$
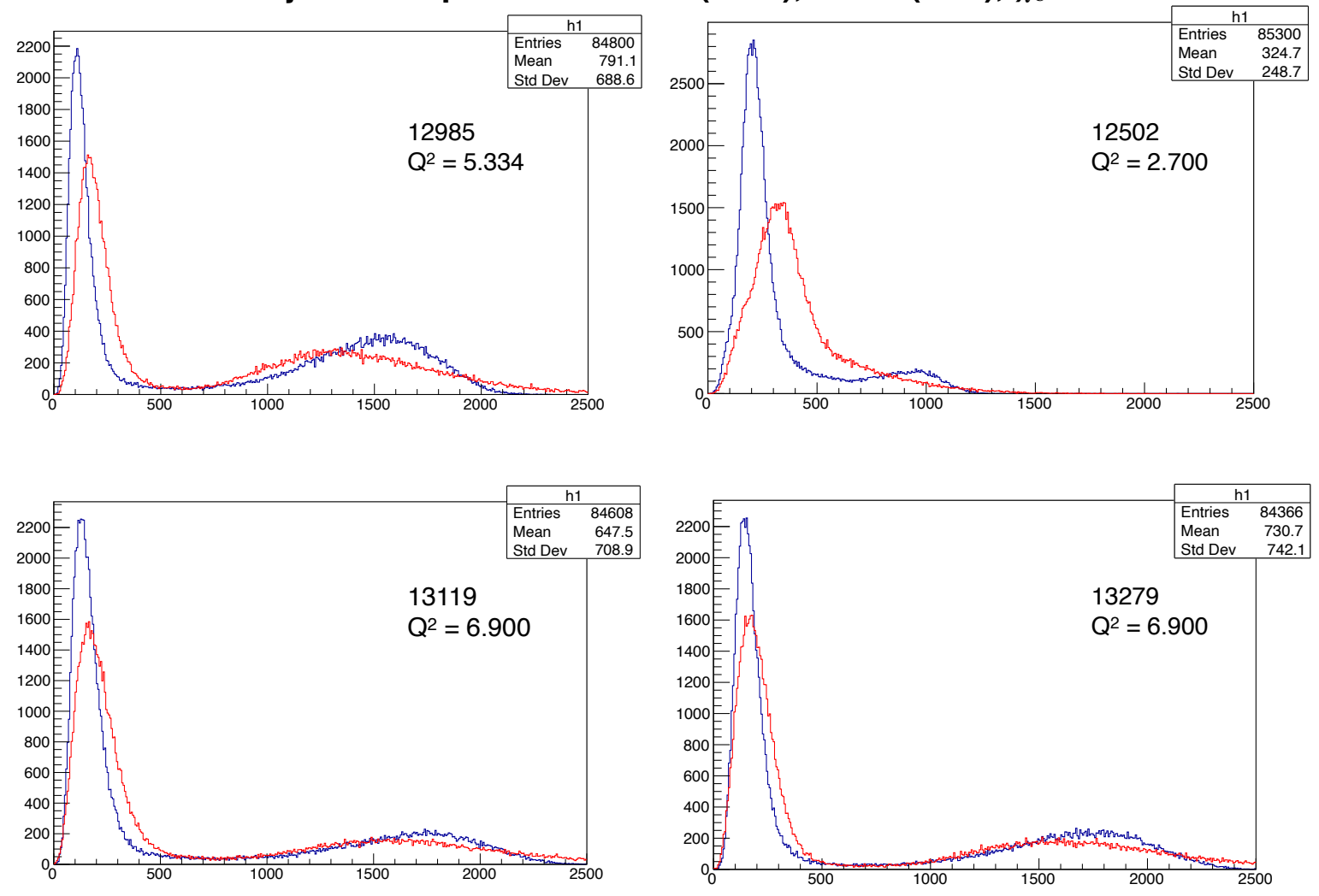

Pion rejector layers for different runs shows the gain mismatch between layers and change with kinematics.

FIG. 19: Figure shows PRL1 sum (Blue) and PRL2 sum (Red) for 4 different runs with 3 different kinematics showing the gain mis-alignment of minimum ionizing peak between layers (around channels 100-200). It also demonstrates that the gain is a function of the kinematic setting as evidence by the different mis-alignment among different kinematic runs and similar mis-alignment between the same kinematics runs. 
Figure 19 shows that after the initial calibration, there is a clear separation between minimum ionizing particles (mostly pions) and electrons. However, the gain values of the two layers is mismatched as evident by the mis-alignment in the Minimum Ionizing Peak of the two layers for 4 different runs across 3 different kinematics, from spring 2016. Since the two layers of the Pion Rejectors are physically identical in size and radiation length, It is reasonable to expect the minimum ionizing peak of both layers to align. The mis-alignment sometimes caused a double peaking in the summed spectrum and a not well defined electron peak.

\subsubsection{PION REJECTOR RE-NORMALIZATION}

To remedy the gain mis-match between the Pion Rejector layers, a re-normalization process was developed using a minimization method, which extracts two weighting factors W1 and W2 for the two Pion Rejector layers, in such a way that the re-normalized full-energy electron peak of the summed spectrum is at spectrum channel 1000.

$$
\text { Normalized PRSum }=\frac{P R L 1}{W 1}+\frac{P R L 2}{W 2}
$$

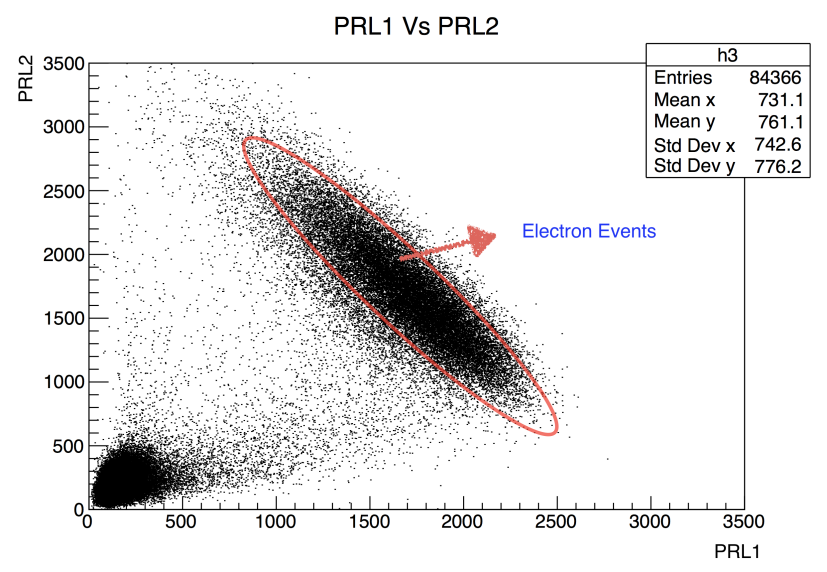

FIG. 20: Amplitude spread of PRL1 Vs PRL2. Red oval is to illustrate the bulk of the single track electron events. these events can be selected and re-normalized using the weighting factors W1 and W2 so that they lie on a 45 degree line and the electron peak of the renormalized Pion Rejector sum is at ADC Chan 1000.

Figure 20 shows the amplitude spread of PRL1 Vs PRL2. The candidate electron events are selected and the variance around the total energy axis (PRL1+PRL2) is minimized to obtain the weighting factors as described in Appendix A. Figure 21 shows the performance 

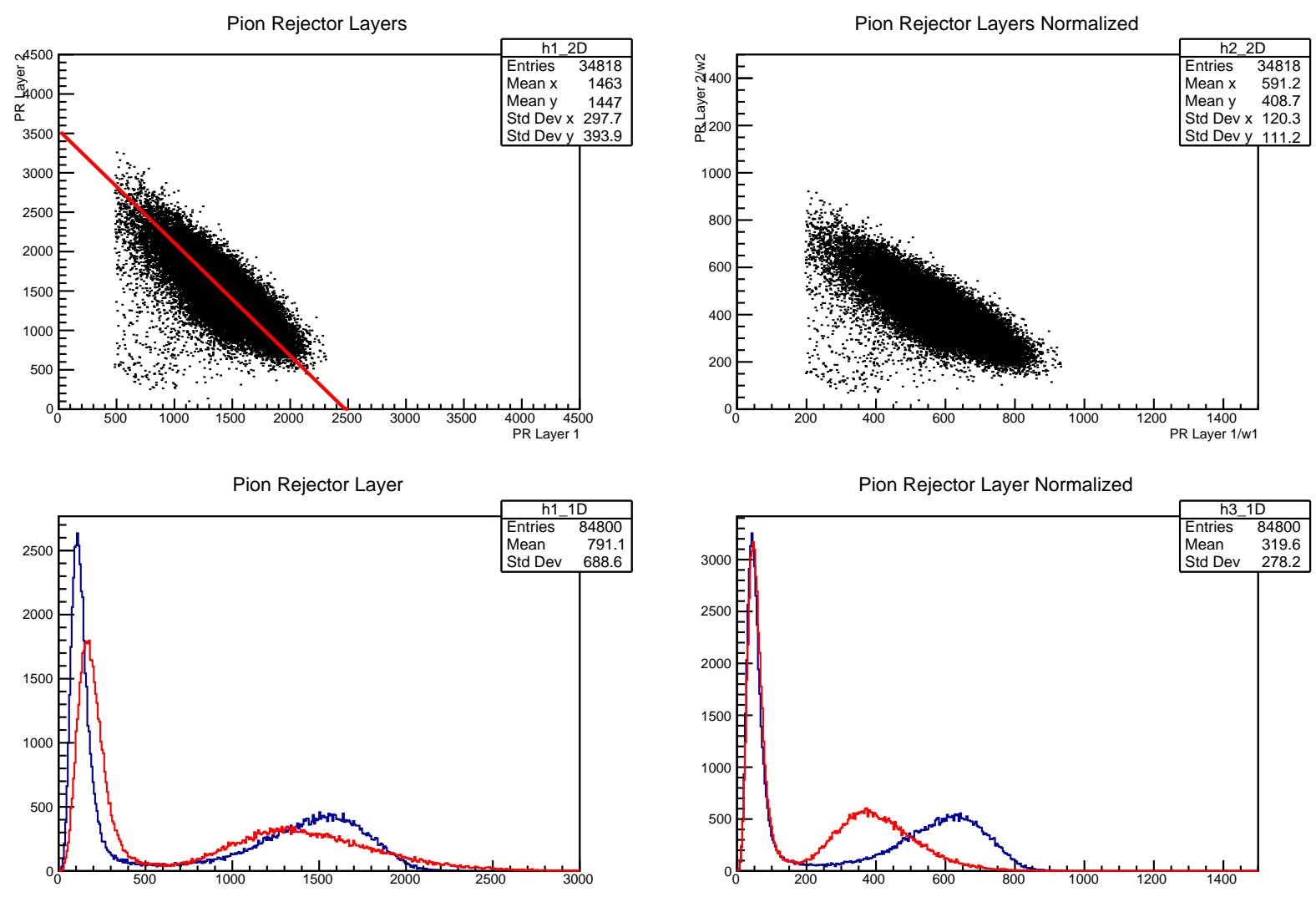

FIG. 21: Figure shows the renormalization for run 12985. Plots on the Left shows 2D and 1D Pion Rejector Spectrum before renormalization and the plots on the right shows the same after renormalization. 1D PRL1 spectrum is given in blue and PRL2 in red. We can see that the renormalization procedure automatically aligns the minimum ionizing peaks of the two layers. 
of the re-normalization on Run 12985. Re-normalization automatically aligns the minimum ionizing peaks of both layers of the Pion Rejector. The normalized electron PRL1 signals are consistently larger than the PRL2 signals, as a consequence of early development of the electromagnetic shower in the first layer while hadronic shower takes time to develop. This can be used to make an additional requirement on the electron selection by requiring the PRL1 to have signals above the minimum ionization. Table 2 summarizes the re-normalization factors for each kinematics based on the averages across all the runs in the kinematic setting. $\sigma_{\mathrm{w} 1}$ and $\sigma_{\mathrm{w} 2}$ are the standard deviation.

\begin{tabular}{|c|c|c|c|c|}
\hline Kin & $W 1$ & $\sigma_{w_{1}}$ & $W 2$ & $\sigma_{W_{2}}$ \\
\hline $36 \_1$ & 2.49 & $1.08 \mathrm{E}-02$ & 3.36 & $3.44 \mathrm{E}-02$ \\
\hline $36 \_2$ & 2.68 & $4.11 \mathrm{E}-03$ & 3.72 & $6.99 \mathrm{E}-03$ \\
\hline $36 \_3$ & 3.33 & $1.54 \mathrm{E}-02$ & 4.52 & $2.14 \mathrm{E}-02$ \\
\hline $48 \_1$ & 1.13 & $1.52 \mathrm{E}-03$ & 1.70 & $5.14 \mathrm{E}-03$ \\
\hline $48 \_2$ & 3.17 & $2.03 \mathrm{E}-02$ & 4.19 & $1.59 \mathrm{E}-02$ \\
\hline $48 \_3$ & 2.28 & $4.43 \mathrm{E}-03$ & 3.11 & $6.30 \mathrm{E}-03$ \\
\hline $48 \_4$ & 2.63 & $8.91 \mathrm{E}-03$ & 3.55 & $1.42 \mathrm{E}-02$ \\
\hline $60 \_1$ & 3.00 & $2.80 \mathrm{E}-02$ & 4.09 & $3.24 \mathrm{E}-02$ \\
\hline $60 \_3$ & 2.64 & $1.84 \mathrm{E}-02$ & 3.65 & $2.74 \mathrm{E}-02$ \\
\hline
\end{tabular}

TABLE 2: This table summarizes the renormalization weighting factors $\mathrm{W} 1$ and $\mathrm{W} 2$ of the two layers of the Pion Rejector for each kinematic setting as the average over all the runs in the kinematic together with the standard deviations.

\subsection{GAS CHERENKOV CALIBRATION}

The threshold Gas Cherenkov described in section 3.3.3 is used to trigger the DVCS events and to provide an additional layer of pion suppression in the analysis. Gas Cherenkov is calibrated by a two fold fit method. In first pass, pedestal values were fitted and in second pass the one photo electron peaks are aligned.

Raw Cherenkov amplitude spectrum of each individual channels were fitted using a two part gaussian and a second order polynomial tail to determine pedestal and single photoelectron peak positions and width. This information was used to verify the calibration. This information was also used later to make a Poisson fit to the calibrated spectrum to check the photo electron yield. Figure 22a shows the fit to the raw spectrum of Cherenkov PMT 4. Table 3 shows the extracted parameters.

Figure $22 \mathrm{~b}$ shows the performance of initial calibration against the second calibrations. 


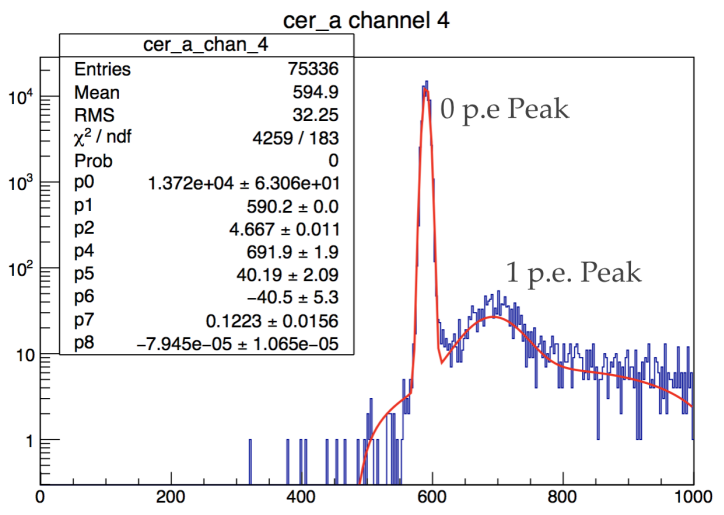

(a) $1^{\text {st }}$ Pass.

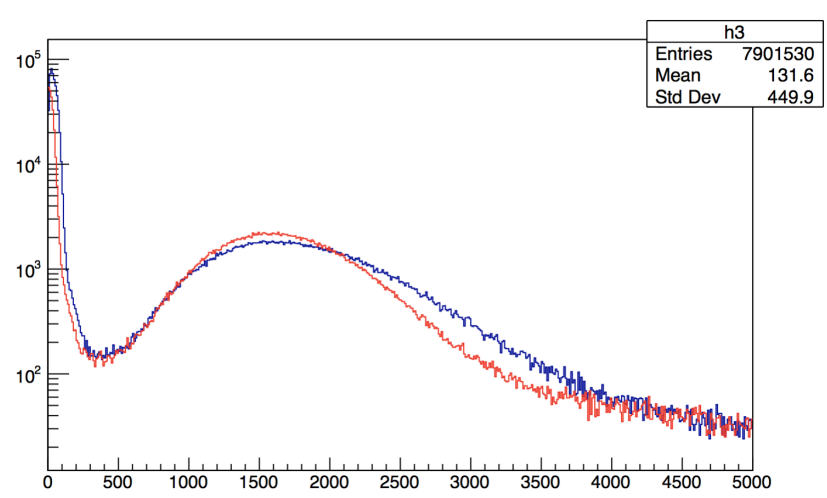

(b) Calibration test

FIG. 22: Each individual photo tube spectrum of the Gas Cherenkov in LHRS were fitted using a two part gaussian and a second order polynomial tail to extract the position and width of pedestal and single photo electron peak. Figure $22 \mathrm{a}$ shows the fit for the $4^{\text {th }}$ photo tube. Figure $22 \mathrm{~b}$ shows Cherenkov amplitude spectrum with two different calibration. Blue spectrum shows the initial calibration performed by the GMp group. Red spectrum shows the calibration performed for verification. The test calibration recovered a cleaner Poisson distribution.

\begin{tabular}{|c|c|c|c|}
\hline & Amplitude & Position & Width \\
\hline Pedestal & 13720 & 590 & 4.66 \\
\hline Single Photo Electron & 21 & 692 & 40.2 \\
\hline
\end{tabular}

TABLE 3: This table shows the extracted calibration parameters for the $4^{\text {th }}$ phototube of the Gas Cherenkov. This parameters are then used to calibrate the Gas Cherenkov and extract the photo electron yield per electron travelled through the Gas Cherenkov. 
Second calibration gives better pedestal subtraction as well as better calibration of the long non-Poisson tail in the spectrum. Yet, for the purpose of particle identification initial calibration would yield a similar performance and hence kept in the analysis process. The long non-Poisson tail, however, is a subject of interest and thought to be from Multi-Electron events. This is discussed in section 4.4 .

\subsubsection{STUDY OF THE EFFECT OF THE WAVELENGTH SHIFTING PAINT}

Cherenkov PMT signals follow Poisson statistics. Fitting the raw Cherenkov PMT signal with the following fit function could be used to determine the photo electron yield of the Gas Cherenkov.

$$
f(i)=N \sum_{n=0}^{\infty} e^{-\mu} \frac{\mu^{n}}{n !} \times \operatorname{Gauss}(i, 100 n, \sigma \sqrt{n}) ;
$$

$i$ - Spectrum channel number,

$N$ - Total number of events,

$100 n$ - Calibrated $n$-photoelectron position, with 1 photoelectron peak at 100,

$\mu$ - Mean number of photo electrons,

$\sigma$ - Width of the 1 photo electron peak.

The Photomultiplier tubes of the Gas Cherenkov are not sensitive to UV light (borosilicate - a UV non-transparent window). During the $12 \mathrm{GeV}$ upgrade of the Jefferson Lab, A Wavelength Shifting Paint (WLS) was applied to the PMTs. In $6 \mathrm{GeV}$ era, before application of the paint, the photo electron yield for electrons traveling through the gas was 6-7 photo electrons [5]. Figure 23 shows the Poisson fit for the central 4 PMTs of the Gas Cherenkov. New photo electron yields for electron events are roughly $14-16$. This is a 50-65\% increment over the $6 \mathrm{GeV}$ era. Initial testing by Allada et al., [6] concluded the WLS gave a moderate $30-40 \%$ increase in the number of photo electrons. In a recently published result, they found more impressive $65 \%$ increase in beam test, which is in agreement with the result observed here in 2014.

Since the Cherenkov ADC spectrum follows a Poisson distribution with mean $\mu$, probability to detect $\mathrm{n}$ photo electrons would be

$$
e^{-\mu} \frac{\mu^{n}}{n !}
$$

With the analysis threshold for electron identification set at 1.5 photoelectrons, the electron detection efficiency $\epsilon$ can be estimated as:

$$
\epsilon=1-(1+\mu) e^{-\mu}
$$



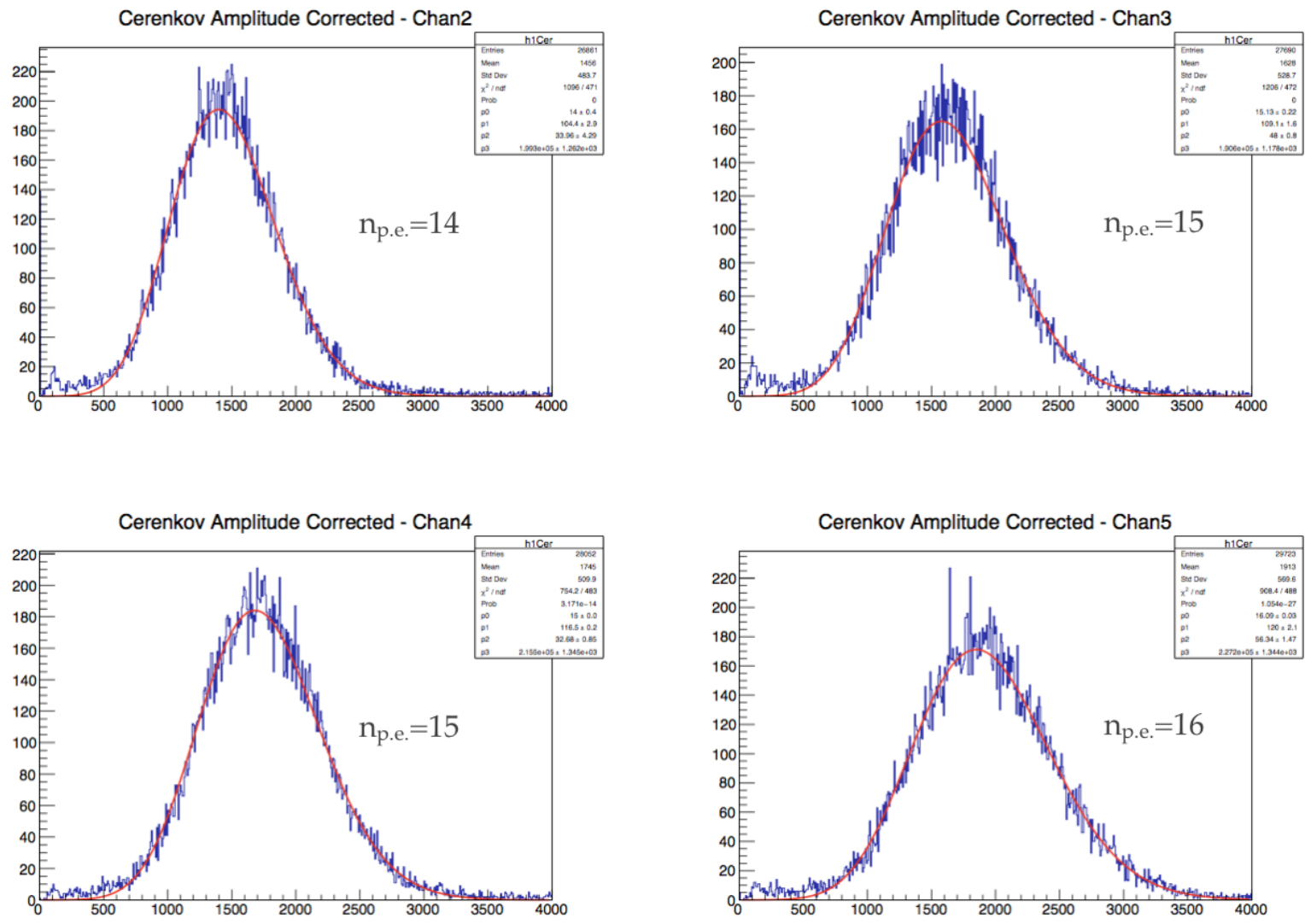

FIG. 23: Poisson fit on central 4 PMT's of the Gas Cherenkov for DVCS efficiency run 10419 on carbon target taken in fall 2014, with scintillator triggers. Here, only the electron events from Pion Rejector with $90 \%$ of the Cherenkov light collected by the PMT in question are selected. Since this selection considers only one out of the 10 phototubes, it also rejects $90 \%$ of the Multi-Electron events seen as a long non-poisson tail in Figure 22b. New photo electron yield is roughly about 14-16 photo electrons. 
Since an electron event yields 14 photo electron, the Poisson estimate of detection efficiency is in excess of $99.99 \%$.

\subsubsection{ELECTRON IDENTIFICATION AND EFFICIENCY}

As mentioned before, a certain degree of pion suppression is built in to the trigger itself. In addition to that, Pion Rejector and Gas Cherenkov in combination are designed to give a pion suppression of a factor of $2 \times 10^{5}$ above $2 \mathrm{GeV} / \mathrm{c}$, with a $98 \%$ efficiency of electron selection in RHRS [5]. LHRS is expected to have a similar performance.
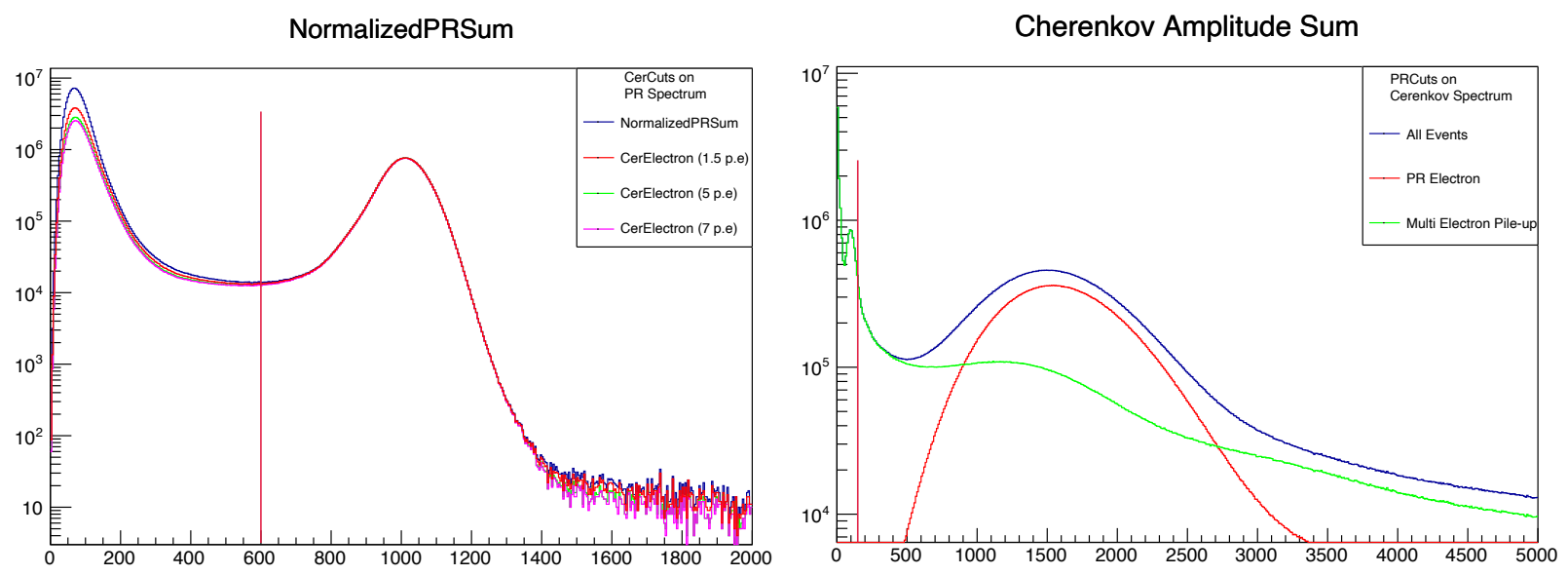

FIG. 24: Normalized Pion Rejector sum with various Cherenkov cuts (left) and Cherenkov spectrum with various Pion Rejector Cuts (right) are shown here. Events that are on the right to the red vertical line on both plots, that is, events with more than 1.5 photo electron in the Cherenkov and NormalizedPRSum greater than $60 \%$ of the full electron peak with more than $20 \%$ of the full electron peak in PRL1 are considered "Good Electron". 1. PE is chan 100 in Cherenkov spectrum. Left plot shows very minimal effect if increasing the cerenkov cut up to $7 \mathrm{PE}$ due to the already high efficiency of the $1.5 \mathrm{PE}$ cut. Right plot shows that the Pion Rejector cut effectively eliminating the multi-electron bacground - the non-Poisson tail.

Figure 24 shows a typical normalized pion rejector spectrum (left) and a Cherenkov spectrum (right) with various possible cuts. The following cuts are defined for electron identification:

$$
\begin{aligned}
\text { CERElectron } & =\text { CerAmpSum }>150 \\
\text { PRElectron } & =(\text { NormalizedPRSum }>600) \cap(\text { NormalizedPRL1 }>200) \\
\text { GoodElectron } & =\text { CERElectron } \cap \text { PRElectron } \\
\text { PRMediumElectron } & =(\text { NormalizedPRSum }<600) \cap(\text { Normalized PRL1 }>200)
\end{aligned}
$$


Here,

1 p.e peak $=$ Chan 100 in Cherenkov

Full electron peak $=$ Chan 1000 in Normalized Pion Rejector Sum

Figure 24(right) shows a long tail in the Cherenkov spectrum extending beyond twice the electron peak. Selecting events with more than $60 \%$ of full energy peak in the Pion-Rejector sum provides strong suppression of this tail. As illustrated by the excellent Poisson fits, this suggests that these events are triggered by more than one low energy electron arriving in coincidence at the Gas Cherenkov. These Multi-Electron events are discussed further in 4.4.2. Figure 24 (left, Normalized Pion Rejector spectrum), together with the "PRMediumElectron" cut shows that the long tail of the PR Minimum Ionizing peak is in coincidence with a background Cherenkov spectrum starting at zero photoelectrons, extending up to 7 photo-electrons before very slowly tapering off above 20 photo electrons. Further investigation shown in Figure 25 reveals that the background events at 0-7 photo electrons are localized to high momentum region of the pion rejector, and totally eliminated by a target cut, which suggests that they could be cosmic radiation, as opposed to leakage of delta rays.
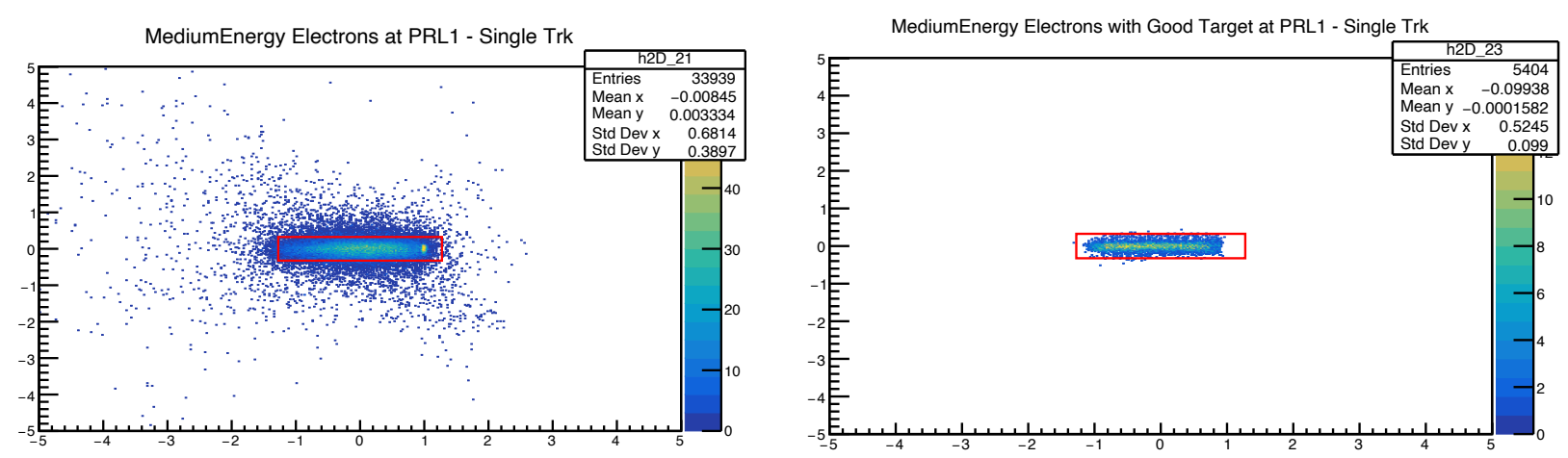

FIG. 25: Left: Medium energy electron pile-up events as selected by CERElectron $\cap$ PRMediumElectron, reconstructed in the geometrical $y$ vs. $x$ plane of the first layer of pion rejector. This spectrum shows a localization at the extreme large $x$ end of the Lead-Glass blocks (which is the region of maximal reconstructed momentum and physically at the top, closer to the ceiling of the Hall). Right: Same events after a target vertex cut, which shows that the cluster of events at large $x$ are not coming from the target itself, an indication that these could be cosmic rays.

\subsection{TARGET VERTEX RECONSTRUCTION AND OPTICS STUDIES}




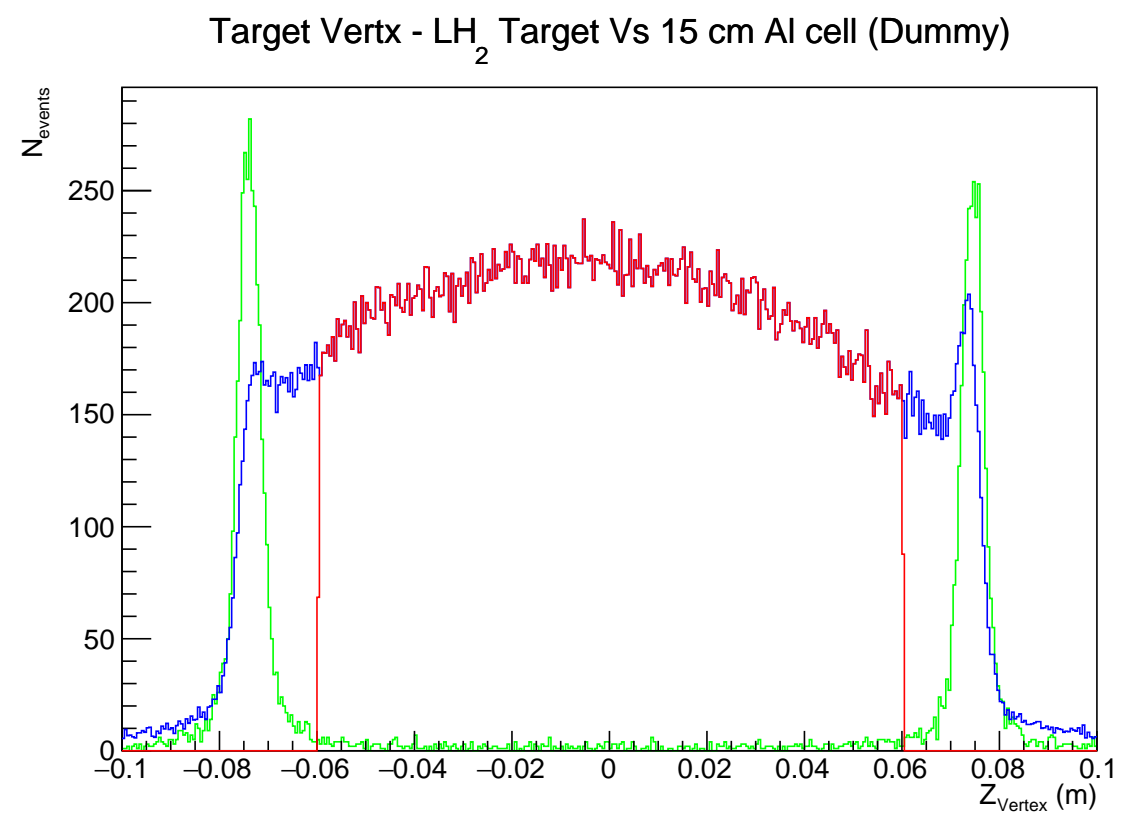

FIG. 26: Figure shows target vertex distributions for Dummy Target run against $\mathrm{LH}_{2}$ target run, normalized to the run duration. Dummy target (Green) shows the scattering from the $\mathrm{Al}$ end caps leaking into $\mathrm{LH}_{2}$ spectrum (Blue). Cutting $1 \mathrm{~cm}$ on each end avoids $\mathrm{Al}$ contamination (Red).

Target system used in DVCS3 Experiment is described in 3.3.1. Hall A provides a dummy target to simulate the effect of $\mathrm{Al}$ end caps of the target holder for the cryogenic targets. Unfortunately, DVCS3 doesn't have dummy data for every desired kinematics. Figure 26 shows a reconstructed target vertex for the dummy target overlaid on hydrogen data for Kin 48_1. Full Width at Half Maximum for the dummy distribution for the kinematics where it is available is around $0.5 \mathrm{~cm}$, indicating cutting $1 \mathrm{~cm}$ on either end of the target would prevent aluminum contamination.

Target center is calibrated during the assembly so that, the spectrometer points to the target center. The reaction vertex is reconstructed as the intersection point of the incoming beam and the extrapolated scattered electron track. The uncertainties in the optics, BPM and raster calibration combined with the minor physical movement of the target center while moving the target and/or the spectrometer or during the cooling and heating of the target could result in an apparent offset of the target center. Figure 27 shows the reconstructed target for 3 different kinematic settings, indicating the changing target offset. 


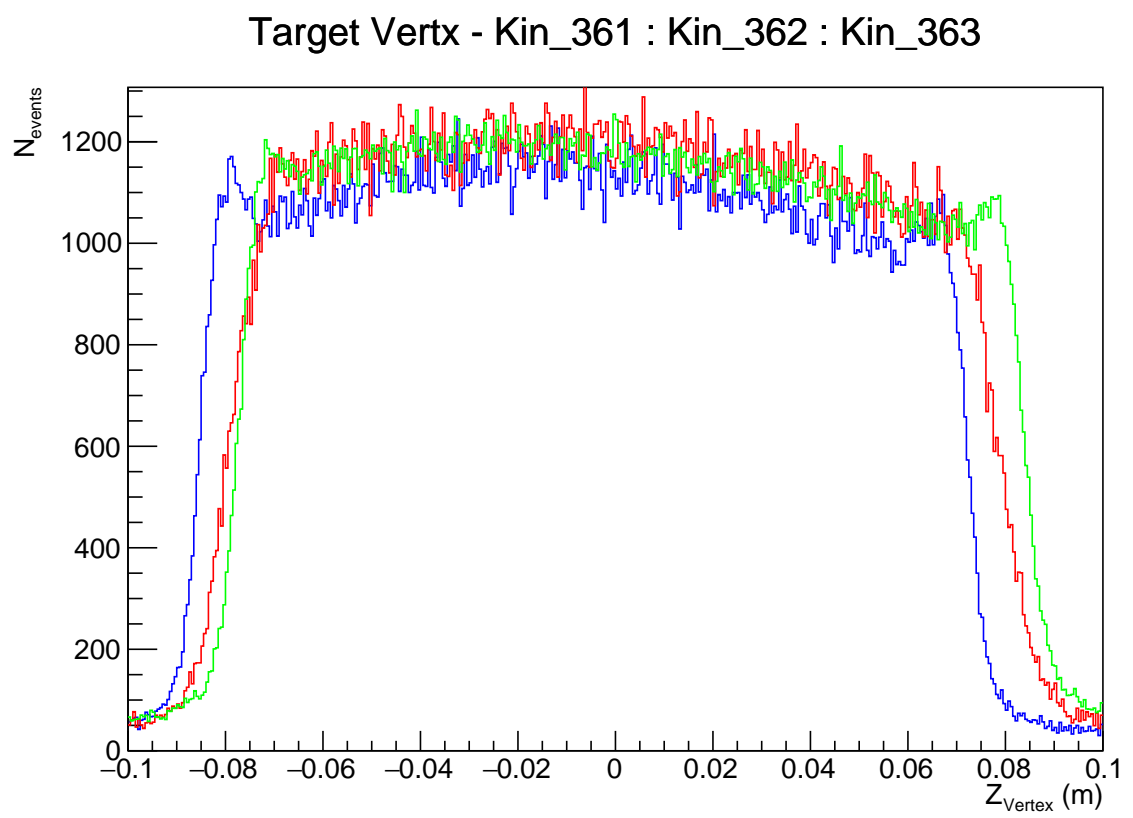

FIG. 27: Target vertex distribution, normalized to the the event count plotted for 3 different kinematic settings, Kin 361 (Blue), 362 (Red), 363 (Green) shows a shift in the target center, kinematic to kinematic.

\subsubsection{OPTICS CORRECTION}

The Q1 magnet of the spectrometer failed to hold full current required for 3 of the 4 kinematic settings during the Spring 2016 run period. For each of this kinematic dedicated optics calibration runs were taken at a small spectrometer angle to increase counting rate and offset the time loss due to extensive calibration runs. Unfortunately, this resulted in the optics data not fully illuminating the focal plane at the larger angles of the DVCS production runs and resulted in larger uncertainties in the higher order terms of the polynomial expansion of the optics matrix. Limiting the polynomial expansion to the $2^{\text {nd }}$ order, while resulting in good reconstruction of the target edges compared to higher order calibration, it resulted in a shorter than $15 \mathrm{~cm}$ reconstructed target. An empirical correction based on rotation $\left(\theta_{\text {rot }}\right)$ 
and re-scaling $\left(\mu_{\text {scale }}\right)$ was then developed to remedy this as follows,

$$
\begin{aligned}
\phi_{t g}^{\prime} & =\phi_{t g} \cos \theta_{\text {rot }}\left(1+\tan ^{2} \theta_{\text {rot }}\right) \\
Z_{\text {vertex }}^{\prime} & =\mu_{\text {scale }}\left\{Z_{\text {vertex }}-\phi_{t g} \tan \theta_{\text {rot }}\right\} \\
y_{t g}^{\prime} & =\frac{X_{\text {vertex }} \cos \theta_{\text {rot }}-Z_{\text {vertex }}^{\prime} \sin \theta_{\text {rot }}}{\cos \left(\arctan \phi_{t g}^{\prime}\right)}
\end{aligned}
$$

Table 4 shows the values of $\mu_{\text {scale }}$ and $\theta_{\text {rot }}$ for Kin 482,483 and 484 .

\begin{tabular}{|c|c|c|}
\hline Kin & $\theta_{\text {rot }}(\mathrm{rad})$ & $\mu_{\text {scale }}$ \\
\hline $48 \_2$ & 0.2290 & 1.056 \\
\hline $48 \_3$ & 0.2372 & 1.053 \\
\hline $48 \_4$ & 0.2051 & 1.042 \\
\hline
\end{tabular}

TABLE 4: Target correction parameters for the 2016 spring data.

Q1 was replaced for the Fall 2016 run period and the replaced magnet was later found to be saturating at higher current. This was not an expected scenario and thus there was no optics data to calibrate, and hence pseudo-data was generated using COSY [59] generated optics matrix for the saturated field and SIMC [8] to perform optics calibration [16].

\subsubsection{TARGET VERTEX CUT}

A target vertex cut of the following form will make sure the events seen in fact are from $\mathrm{LH}_{2}$, taking in to account the target offset and optics corrections,

$$
a b s\left(Z_{\text {vertex }}-Z_{\text {Offset }}\right)<0.065
$$

Here $Z_{\text {vertex }}$ is the optics corrected target vertex. The target offset parameter $Z_{\text {Offset }}$ was computed for each spectrometer setting, by eye as summarized in Table 5. A systematic method to determine the center was developed by Frederic Georges, using the center foil of the optics target. In this, the offset parameter is taken to be the center foil position of the optics target. While this method provides reproducibility, there is no guarantee that the center foil of the optics target and the hydrogen target are aligned at the operating temperatures of the cryogenic target. A comparison between the event selection performance of the two calculations showed that the uncertainties in selected number of events are negligible and hence the optics target positions are kept in the final analysis for reproducibility.

Figure 28 shows the performance of the target cut on each kinematic setting. 


\begin{tabular}{|c|c|c|}
\hline Kin & $Z_{\text {Offset }}(\mathrm{m})$ & $Z_{\text {Offset }}-$ Optics \\
\hline $36 \_1$ & -0.005 & -0.0047 \\
\hline $36 \_2$ & 0.0025 & 0.0025 \\
\hline $36 \_3$ & 0.0050 & 0.0046 \\
\hline $48 \_1$ & 0.0000 & 0.0003 \\
\hline $48 \_2$ & 0.0025 & 0.0017 \\
\hline $48 \_3$ & 0.0025 & 0.0034 \\
\hline $48 \_4$ & 0.0050 & 0.0039 \\
\hline $60 \_1$ & 0.0050 & 0.0040 \\
\hline $60 \_3$ & 0.0025 & 0.0033 \\
\hline
\end{tabular}

TABLE 5: Target offset values for each kinematic setting. $Z_{\text {Offset }}$ - Optics is used in the analysis.
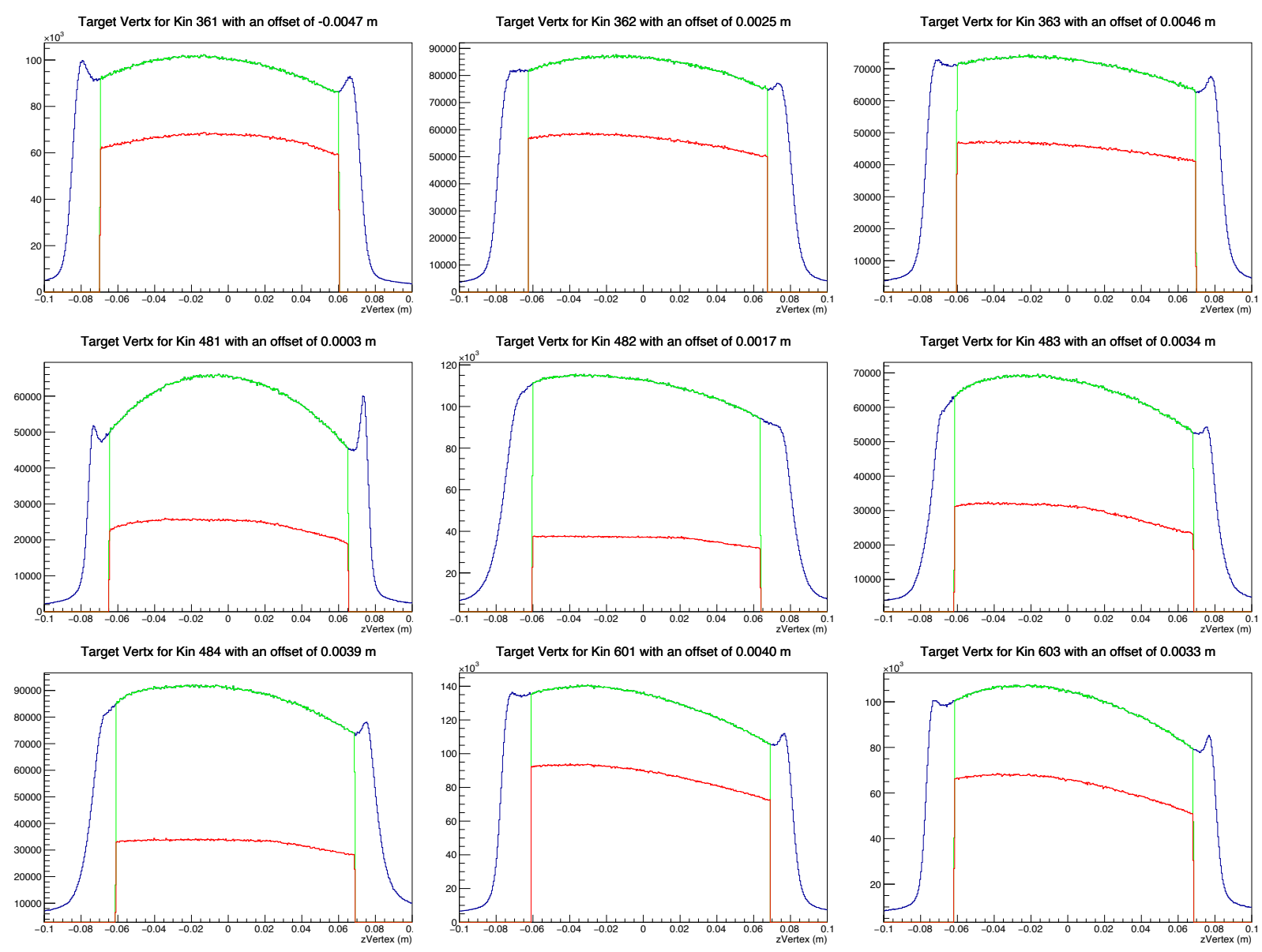

FIG. 28: Target vertex cut for all kinematic settings. Blue is the reconstructed target, Green is the Target Cut and Red is the selected events after applying all electron selection cuts. 


\subsection{TRACKING STUDIES AND TRACKING EFFICIENCIES}

DVCS production data includes about 5\%-10\% of events with more than one reconstructed track in the Vertical Drift Chambers (VDC), with varying accuracies of track reconstruction. The dominant single contributor to multiple scattering was thought to be the horizontal $127 \mu \mathrm{m}$ Ti window at the exit of the spectrometer vacuum [5]. A simulation study in 2005 [65] concluded that, the tracking efficiency due to random coincidences does not impact the reconstructed single tracks, even though the effect increased with increasing number of reconstructed tracks. In traditional practices, events with more than one reconstructed tracks are excluded from the analysis and corrected for at the end assuming both single track and multi track events are equally likely to be good events. In this work I show that the inefficiencies in track reconstruction could also be extended to some classes of events with only one reconstructed track, depending on the occurrence of multiple clusters in some wire planes and develop ways to better handle them in the analysis.

\subsubsection{TRACK RECONSTRUCTION : TRACKING ALGORITHM AND PER- FORMANCE}

Tracking information in Hall A HRS is provided via a pair of Vertical Drift Chambers discussed in 3.3.3. Here I discuss the tracking algorithm in PodAnalyzer v1.5, the Hall-A analysis software used by the DVCS experiments for data reconstruction. A single nominal track in VDC would activate 4-6 wires in each wire plane, forming a cluster. Figure 29 shows an example of a cluster. A cluster is allowed to have maximum of one missing hit or wire gap. At least one cluster per wire plane is required to reconstruct a track. Timing signals from TDC's are converted to drift distances. A local cross-over point $Q i$ and a Local trajectory angle $\theta_{Q i}$ are calculated using a linear fit to drift distances. Since all the algorithm shortcomings are applied to all wires identically, $Q i$ is algorithm independent while, $\theta_{Q i}$ is not. Local cross over points from both the drift chambers are used to obtain global trajectory angles $\Theta_{v}$ and $\Theta_{u}$ (corresponding to the coordinate axes of the wires in each plane) which are algorithm independent. These global angles are then used to project V-plane onto U-plane and vice-versa in each VDC chambers thus giving four tracking coordinates $\left(Q u, Q v, \Theta_{u}\right.$, $\left.\Theta_{v}\right)$. Recalculated co-ordinates of the lower plane represents the reconstructed focal plane track [32].

When multiple clusters occur in a single wire plane, the current algorithm employs following methods to best match the clusters. 


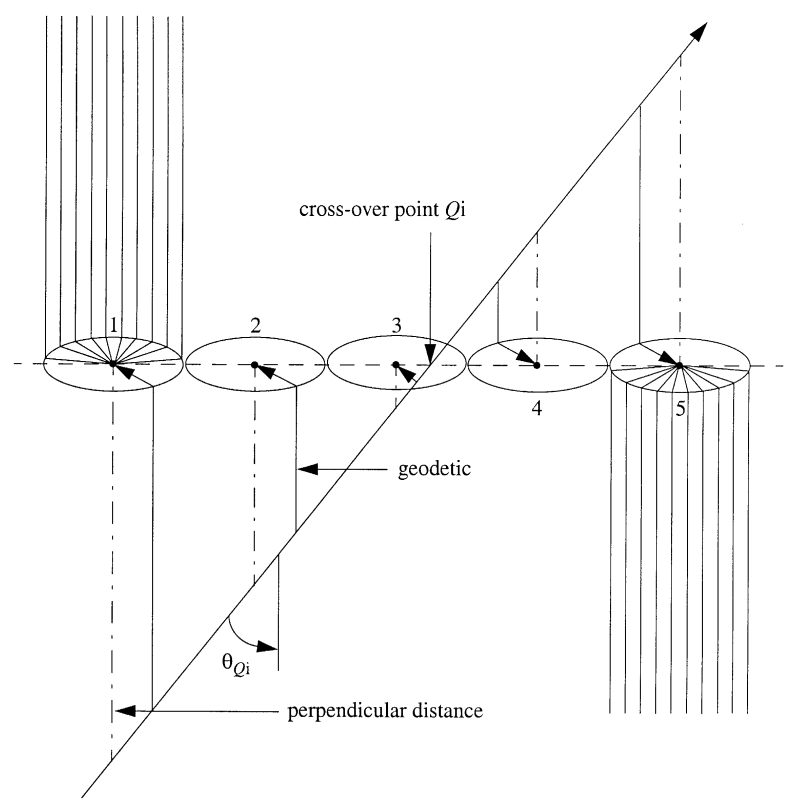

FIG. 29: Each nominal track activates around 4-6 wires in a single wire plane (clusters). $\theta_{Q i}$ is local drift angle and Qi is local cross over position

- UV Matching : Trying to pair U and V clusters of a single VDC chamber by matching pivot wire drift time.

- BT Matching : Trying to match clusters from top and bottom chamber algorithmically by an assigned error value. Each UV cluster pair in a single VDC chamber is considered to project a track onto the other chamber. Then the distance between the local pivot point and the projected point is calculated in each chamber $\left(d_{B}, d_{T}\right)$. The sum of the squares of each pair of distances is considered the error $\left(E=\sqrt{d_{B}^{2}+d_{T}^{2}}\right)$ and the best BT pair is selected based on the least error. This process is then repeated excluding the selected cluster pair to find more pairs and hence tracks. Each track has an overall $\chi^{2}$ based on the differences between track position and drift distance.

The above methods have following caveats [40].

- Pivot wire drift time matching doesn't guarantee that the clusters with similar drift times are made by the same track and hence essentially random, there are no true correlations. 
- UV pairs outside of the active chamber area are not rejected.

- BT Matching only excludes the cluster pair from further matching, not the individual clusters leading to double counting of the clusters. This would mean, the reconstructed tracks are not necessarily represent individual particle tracks, they are merely track candidates. There are no error value cut-off to limit the matched pairs.

Track Reconstruction accuracy varies depending on the number of clusters in each wire plane. I use the notation $\left(n_{\text {clust } U 1}, n_{\text {clust } V 1}, n_{\text {clust } U 2}, n_{\text {clust } V 2}\right)$ to indicate the number of clusters in each wire plane with $X$ being more than one clusters. I here summarize Ole Hansen observations from his studies on tracking performance [40].

- 0M4SClstTrk $(\mathbf{1}, \mathbf{1 , 1}, \mathbf{1})$ : Golden Single Track. Most accurately reconstructed track. This class of events still could have reconstruction inefficiencies due to accidentals within the constraints of VDC design limitations and current algorithm.

- 1M3SClstTrk $(\mathrm{X}, 1,1,1),(1, X, 1,1)$ and so on: When more than one cluster is present only in one of the four wire planes, a correct track is almost always found.

- 2M2SClstTrk $(2,2,1,1)$ or $(1,1,2,2)$ : More than one cluster in both wire planes of one chamber and only one cluster each in both wire planes in the other chamber also yields a single reconstructed track, but this time the accuracy of the reconstructed track might not be as high as other two cases.

- MultiTrack: Any other combination of clusters yield more than one reconstructed track, again with varying accuracies of reconstruction based on the combination of the number of clusters and the chambers and/or wire planes of occurrence. Following are few examples,

$(\mathrm{X}, \mathbf{1}, \mathrm{X}, \mathbf{1})$ and $(\mathbf{1}, \mathrm{X}, \mathbf{1}, \mathrm{X})$ - More than one track with the possibility of one track being a good track

$(1, X, X, X),(X, 1,1,1)$ and so on - More than one track with the possibility of all of them being bogus

$(\mathbf{X}, \mathbf{X}, \mathbf{X}, \mathbf{X})$ - Multiple track with a falling probability of a correct track (Track Candidates)

Following nomenclature is used in this text 
- SingleTrack : All events with a single reconstructed track (GoodSingleTrack $\cup$ MultiClusterTrack)

- GoodSingleTrack : 0M4SClstTrk U 1M3SClusttrk

- MultiClusterTrack : 2M2SClustTrk, even though MultiTrack events are technically Multi Cluster Events, for simplicity of the text, MultiClustTrk refers to 2M2SClstTrk. MultiTrack : Events with more than one track candidates.

\subsubsection{NATURE AND ORIGIN OF MULTI CLUSTER EVENTS}

An additional structure near the path of a nominal track, is the Quadrupole magnet Q3. It is possible that some high energetic electrons, shower from the edges of the Q3 magnet, resulting in secondary electrons whose trajectories bend in the fringe field of Q3, ending up in the nominal spectrometer acceptance. The edge of the cryostat containing the coil is roughly at $z=-3.57 \mathrm{~m}$ in transport coordinates.

Figure 30 shows the track reconstruction at Q3. It shows a localization around ( $X=$ $\pm 0.3, Y=0, Z=-3.57)$ indicating possible showering from the Q3 Magnets. Even though the reconstructed single track events do not show any such localization, the wings along the diagonal follows a similar pattern to that of the Multi Track events. This suggests that, these events could be good single track electron events but corrupted in the track reconstruction due to a showered secondary electron producing a cluster in random coincidence.

Figure 31 shows the track reconstruction at $z=-3.57 \mathrm{~m}$ for different classes of single track events. Events with only single clusters in all 4 wire planes of the VDC (0M4SClustTrk) is the most accurately reconstructed track by the current reconstruction algorithm [40]. High energy electron events of this class, reconstructed outside of the acceptance could still contain a portion of good but corrupted events. Comparing this class of events with 1M3SClustTrk events, where one of the 4 wire planes has a multi cluster and the other 3 wire planes have single cluster, does not show a huge efficiency drop while the 2M2S events, where both wire planes of one VDC chamber has 2 clusters each and both wire planes of the other chamber has only one cluster each, clearly shows a huge efficiency drop.

From above, one could draw the following conclusions.

- A large chunk of multiple clusters in VDC wire plane could have the origin of secondary showering at Q3 Magnet 

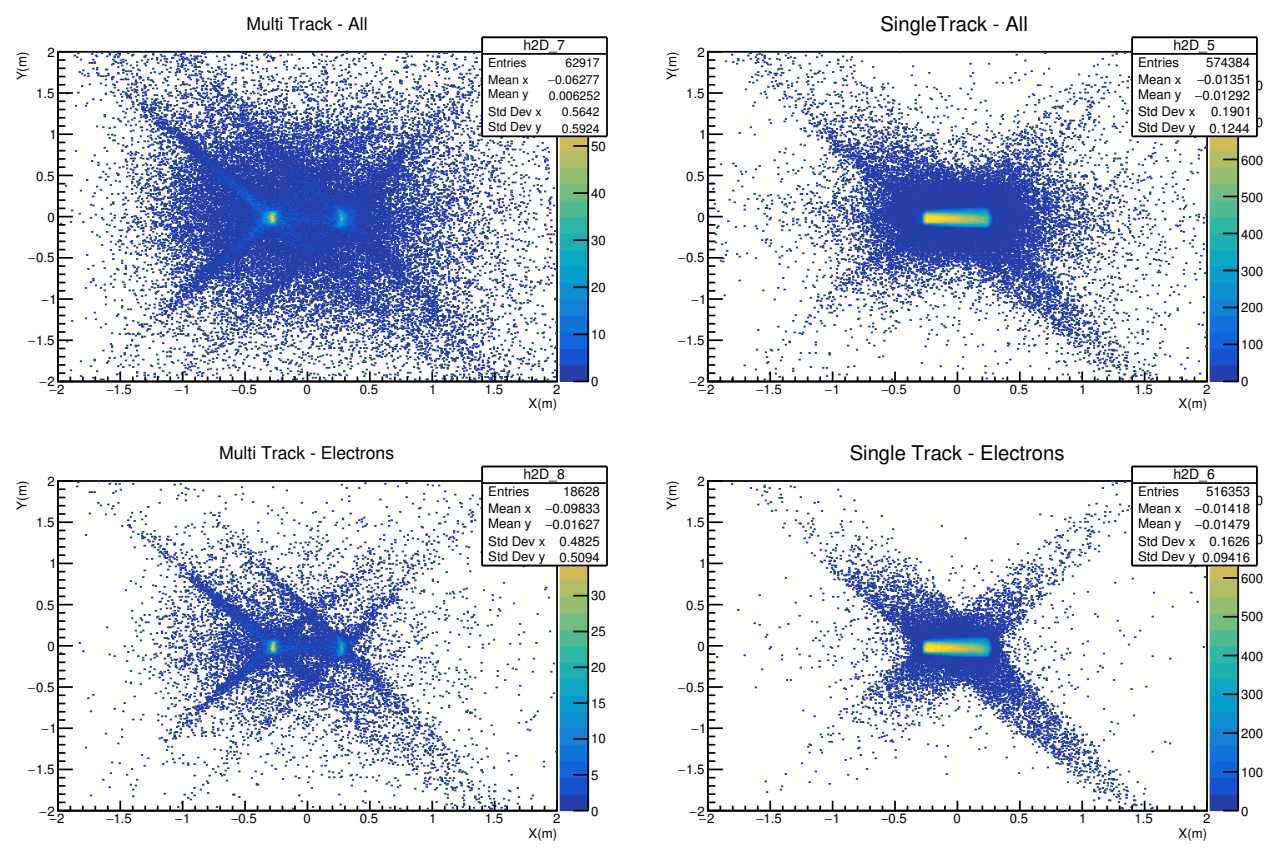

FIG. 30: Track Co-Ordinates reconstructed at Q3 Magnet for Run 14228 of Kin 36_2. Plot on top left shows the $1^{\text {st }}$ reconstructed track for all the events that have more than one reconstructed track. Top right shows the track co-ordinates for only events with one reconstructed track. Bottom plots shows the same plot for identified electron tracks only. Electron tracks are selected based on gas Cherenkov and Pion Rejector. The localization around $Y=0$ and $X= \pm 0.3$ together with the crescent shape suggest showering from the edges of the Q3 magnet 

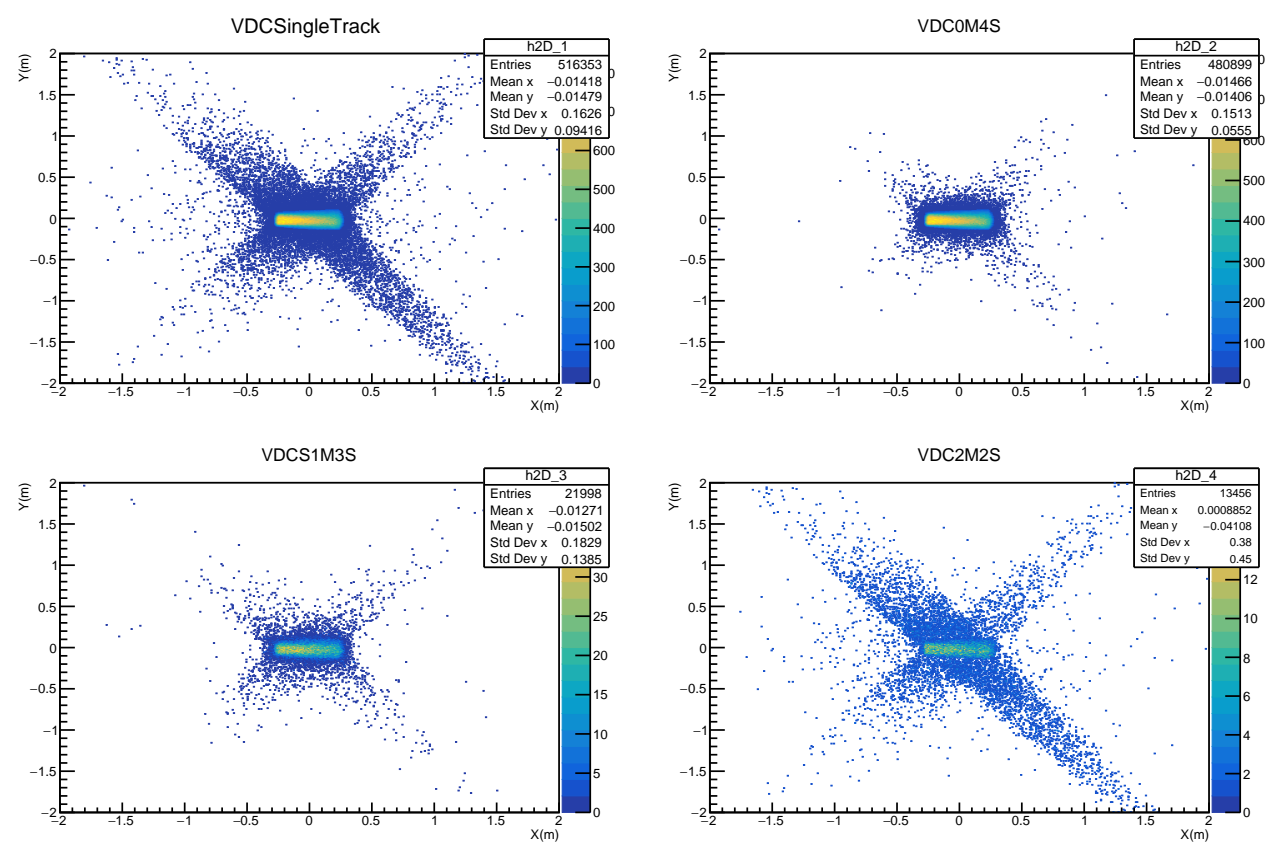

FIG. 31: Track Co-Ordinates reconstructed at Q3 Magnet for Run 14228 of Kin 36_2 for different classes of single track events with good electron. Plot on top left shows the track co-ordinates for all events with only one reconstructed track. Top right shows the events with all single clusters in every wire plane of the VDC. Bottom left plot shows the event with one multiple cluster in one of the wire plane and single clusters in all other three wire planes. Bottom right shows the events with purely single cluster on both wire-planes of one chamber and 2 clusters each on both wire planes of the second chamber. Electron tracks are selected based on gas Cherenkov and Pion Rejector. Even though there are no clear localization here as in Figure 30 its shows evidence that the tracking efficiency reduces with increasing number of clusters in wire plane 
- These low energy secondary electrons could make a cluster in random coincidence with a good electron track and confuse the tracking algorithm

- based on the number of these random clusters, and the wire plane they are produced, the algorithm could have varying reconstruction efficiency for different classes of events.

\subsubsection{TRACKING EFFICIENCIES AND IMPACT ON DATA ANALYSIS}

There are 3 different ways an inefficiency could occur in track reconstruction

- Chamber resolution and Angle reconstruction : This would be an inherent inefficiency of the VDC system and would affect all classes of events in the same way

- Random Electronic Noise : A random electronic noise sometimes could register a cluster and make a random coincidence with other clusters. These would mostly impact 0M4S and 1M3S classes of events.

- A random coincidence of the background shower : These are the background cases discussed in section 4.4.2. Effect of the background shower would vary depending on the number of clusters registered and yield different inefficiencies between different classes of events. 2M2SClustTrk events and MultiTrack events are very much susceptible to these types of inefficiencies.

A poorly reconstructed track, when propagated back to the target, might not appear as originating from the target regardless of whether it's a good event from the target or not. As a result, these events might be eliminated upon applying a target cut. Also, this will affect the different classes of events differently as seen above.

Figure 32 shows the tracks reconstructed at the pion Rejector Layer 1. All events shown here are passing the Particle ID cut and hence expected to be within the boundary of the Pion Rejector, even though a large portion of these events are (poorly) reconstructed to be missing the Pion Rejector by considerable distance. Applying the acceptance cut will eliminate these events completely.

Following quantity, could give us an indication of the impact of acceptance cut on these events.

$$
\text { Accepted Event Fraction }=\frac{N_{\text {AcceptancenElectronID } \cap \text { Track class }}}{N_{\text {ElectronID }}}
$$



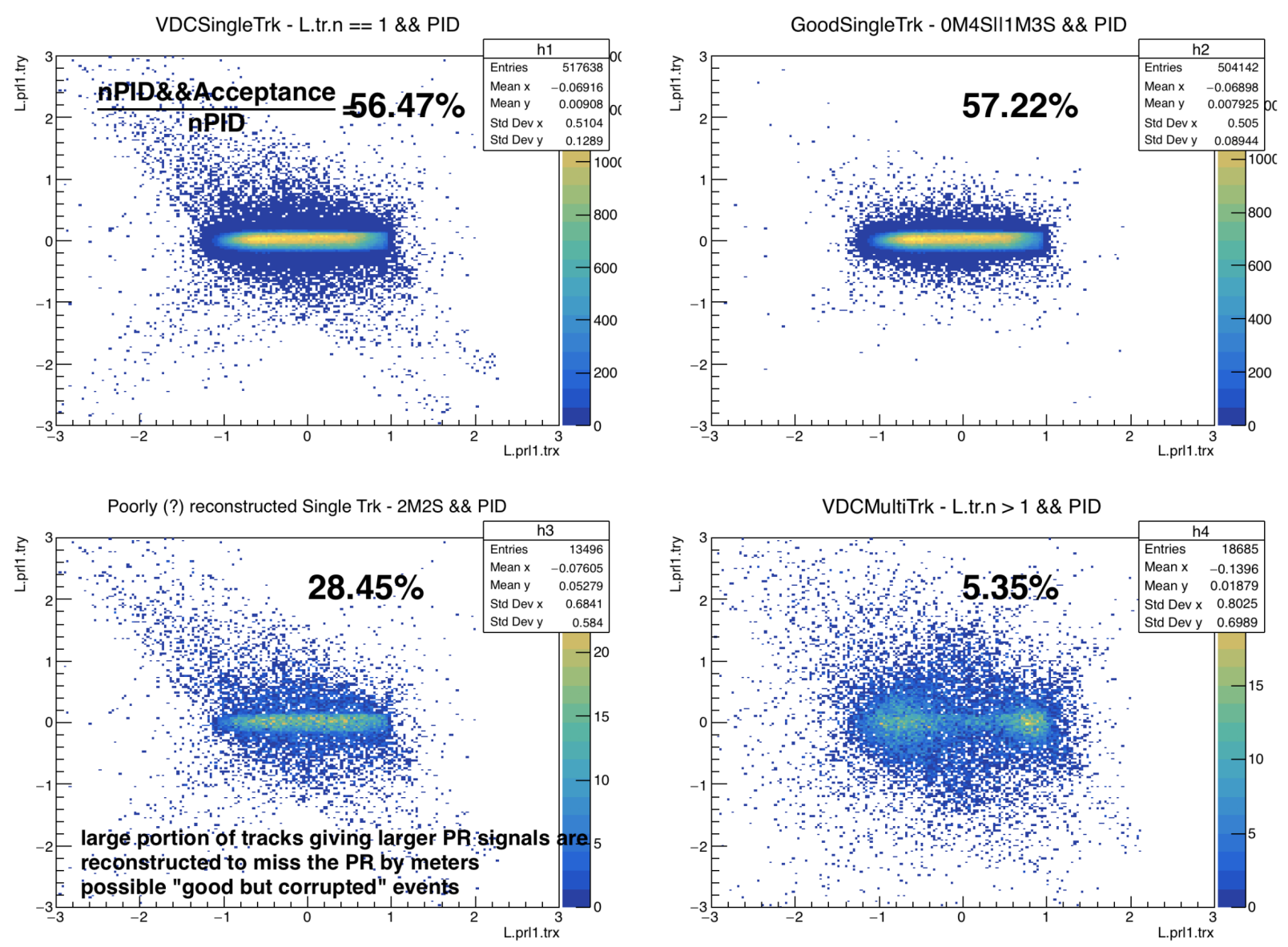

FIG. 32: Track Co-Ordinates reconstructed at Pion Rejector Layer 1 for Run 14228 of Kin 36_2 for different classes of events with good electron (passing the PID cuts). These are events depositing at least $60 \%$ of full electron peak energy in the Pion Rejector and hence expected to be within its boundary. Plot on top left shows the track co-ordinates for all events with only one reconstructed track. Top right shows the events with at most one multi clusters in any of the wire plane of the VDC. Bottom left shows the events with purely single cluster on both wire-planes of one chamber and 2 clusters each on both wire planes of the second chamber. Bottom left plot shows the events with more than one reconstructed tracks. The accepted event percentage from equation 55 for each event class is shown on the plot. acceptance drops to nearly half for $2 \mathrm{M} 2 \mathrm{~S}$ events from the other two types of single track events

\begin{tabular}{|c|c|c|c|c|}
\hline EventClass & SingleTrk & $0 M 4 S \cap 1 M 3 S$ & 2M2S & MultiTrk \\
\hline Accepted Event Fraction & $56.46 \%$ & $57.22 \%$ & $28.45 \%$ & $5.35 \%$ \\
\hline
\end{tabular}

TABLE 6: Accepted Event Fraction values for different classes of events for Run 14228 of Kin 36_2 shows the effect of varying tracking efficiency with different classes of events 
Table 6 shows accepted Event Fraction, that is, the fraction of good electron candidates (selected by "GoodElectron" cut) that pass acceptance cut (R-Function and Target Reconstruction cuts) for each classes of events for the Run 14228 of Kin 36_3. While about 60\% of GoodSingleTrack events are accepted, only a 30\% of 2M2S events are accepted. This indicates the different classes of events will be impacted differently by the acceptance cut due to the inefficiencies in track reconstruction introducing an error in the final result. Since the

effect is very prominent in 2M2S events and events with multiple track candidates, keeping them in the analysis and applying the acceptance cut will eliminate a very large portion of good but poorly reconstructed events. Track reconstruction inefficiencies impact 1M3S events and 0M4S events in almost similar manner, without any real distinction between them.

Following conclusion can be made from the above,

- Highest efficiency for track reconstruction occurs for 0M4S events and 1M3S events. Together these two classes of events makes the GoodSingleTrack events and can be kept in the analysis after the acceptance cut

- Track reconstruction efficiencies drops drastically for any other type of Multi Cluster events. To better handle the effect, these type of events should be excluded from the analysis and corrected for at the end.

\subsubsection{EVENTS WITH MULTIPLE TRACK CANDIDATES VS ACTUAL MULTI PARTICLE TRACK EVENTS}

Track reconstruction algorithm reconstructs up to 9 tracks per event depending on the number of multi clusters on the individual wire planes of VDC chambers. The way the cluster matching works in the current algorithm results in these reconstructed tracks being mostly track candidates rather than guaranteed individual particle tracks. These reconstructed tracks are ordered in the event tree by increasing error value calculated in the BT Matching. Tracking efficiency falls with increasing number of reconstructed tracks [65]. This could occur due to either tracking algorithm struggling to BT match the correlated clusters or due to the obvious limitation of not excluding the individual clusters from further matching. A previous study found that, events with 3 or more reconstructed track to have a tracking efficiency bellow $80 \%$, while single track and two track events have efficiencies greater than $90 \%$ [65] 


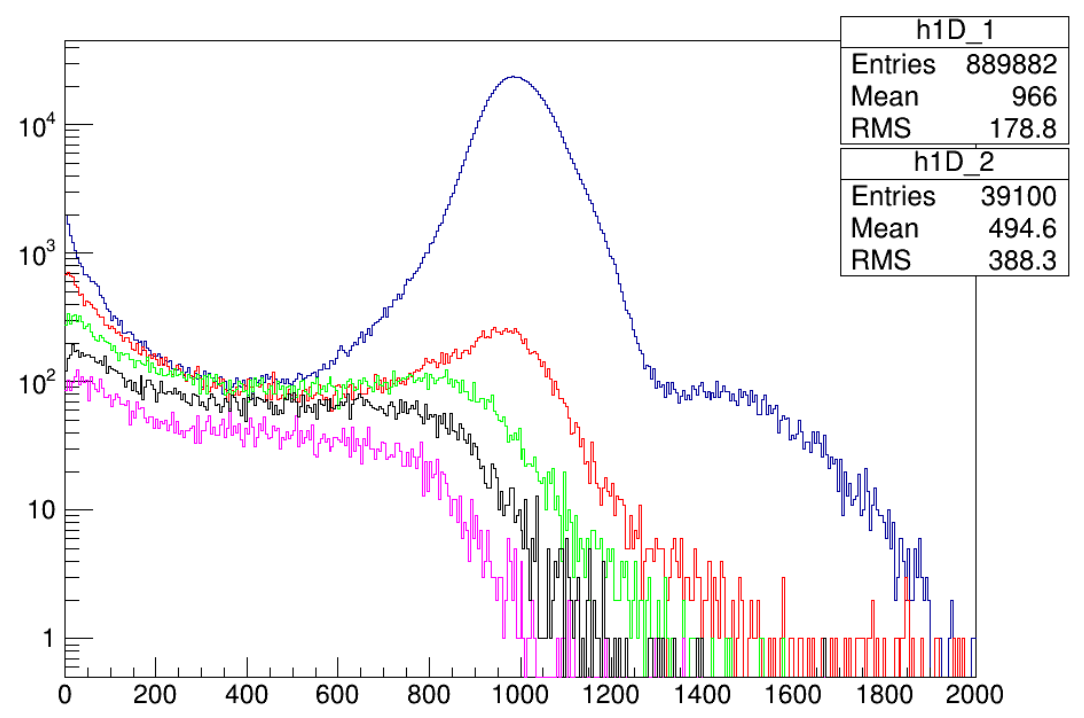

FIG. 33: Pion Rejector sum for single track (blue), 2 track candidate(red), 3 track candidate (green), 4-track candidate (black) and 5 track candidate (purple) events in log scale. Only 2 track events are showing a considerable electron peak.

Figure 33 shows the Normalized Pion Rejector layer sum for varying number of reconstructed tracks. The absence of any full energy electron peaks for events with more than 2 track candidates suggests that these are events with a pile-up of low to medium energy shower particles confusing the track reconstruction of the tracking algorithm resulting in many reconstructed track candidates. If these reconstructed tracks are really multiple particles, they would deposit energies in separate clusters in the pion rejector. the next section develops a cluster finding algorithm to search for multi particle events among these track candidates.

\subsubsection{PION REJECTOR CLUSTER ENERGIES AND CLUSTER FINDING ALGORITHM}

The idea here is to develop an infection algorithm to find the clusters and then sum the cluster energies instead of total energy in the pion rejector layers. Weighted amplitudes of each corresponding crystal in both layers of the pion rejector were summed. Then the algorithm was implemented in two iterations. In first iteration, it scans through all blocks 
of the summed pion rejector layers and finds the block with highest amplitude among its neighbors, and flags it with a cluster number. If two of the neighbors has same amplitude, the first one gets the flag. In the second iteration, the algorithm assign the neighboring blocks to the clusters, if one block shares two neighbors with cluster flags, that block is assigned to the cluster which has the largest amplitude. To avoid summing up the electronic noise in each phototube, each block assigned to a cluster was required to pass through a threshold value. Figure 34 shows a raw and an identified cluster of a multi cluster event.
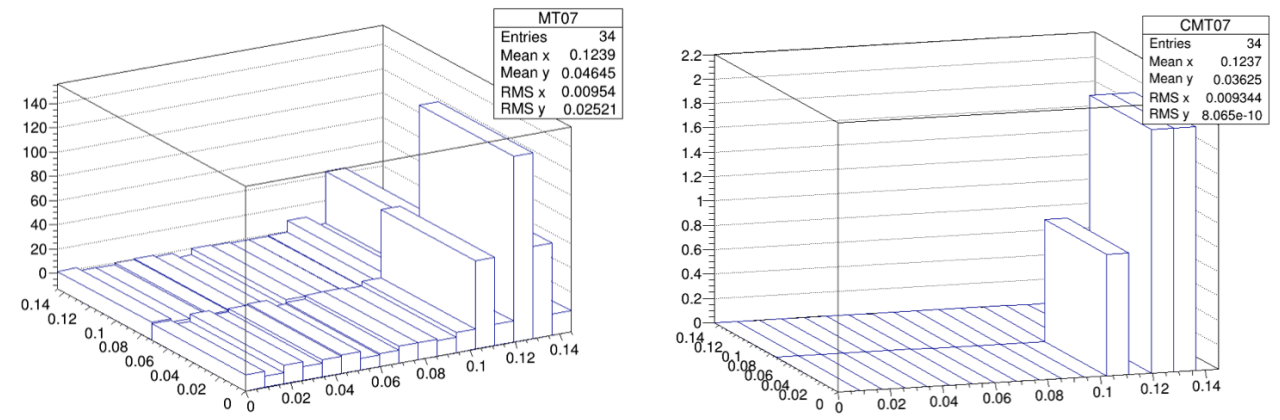

FIG. 34: Left plot shows the raw distribution of a sample event with two clusters in the pion rejector. Right plot shows an identified two cluster event after the $2^{\text {nd }}$ iteration of the cluster finding algorithm.

Table 7 gives the extracted track correction factors for two separate runs from 2010 run period with electron identification using raw sum vs cluster sum of Pion Rejector. Uncertainties of up to $6 \%$ for Multi Track events with more than 2 tracks and very moderate $1 \%$ for two track events were found.

This indicates that the overwhelming majority of the two track events in the data stream are just single track events confused by the algorithm resulting in a second track candidate rather than actual two particle events. Chances of the existence of an actual secondary track increases with increasing number of track candidates explaining the larger uncertainty seen here.

\subsubsection{TRACKING EFFICIENCY CORRECTION}

As previously mentioned, only GoodSingleTrack events are kept in the analysis. Events 


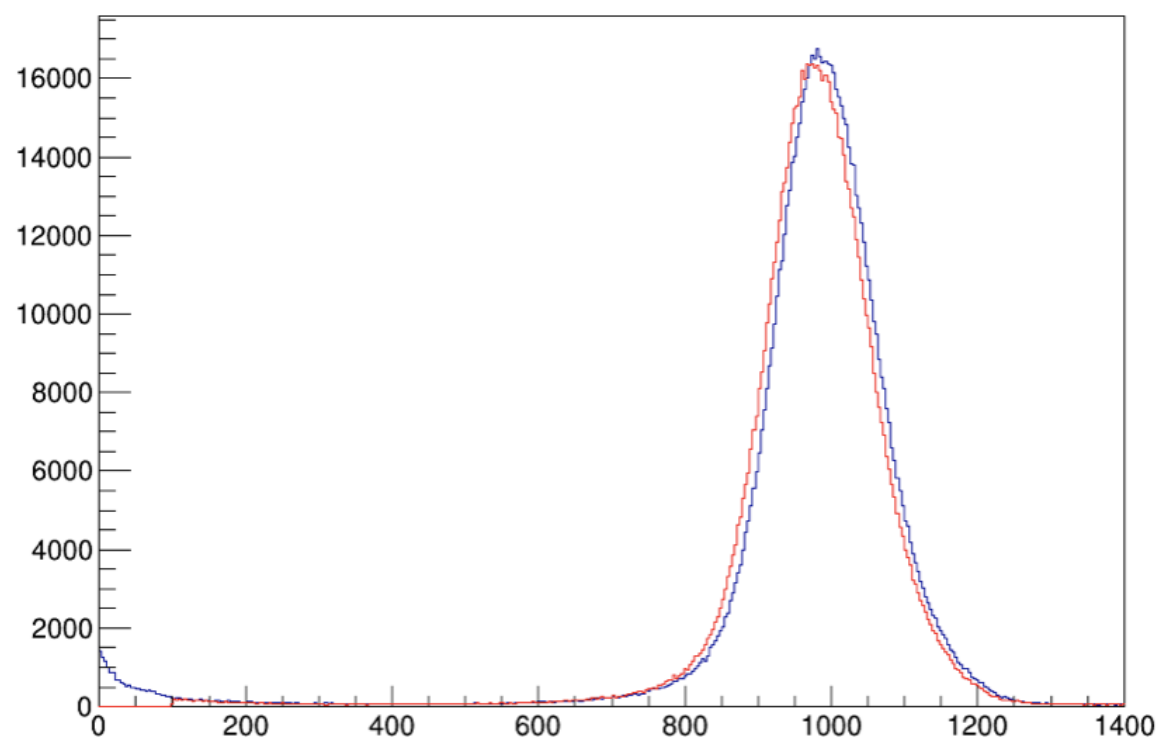

FIG. 35: Plot shows Normalized Pion Rejector sum spectrum of raw sum in blue and cluster sum in red for single track events.

excluded from the analysis should be accounted for. This could be accomplished by applying a correction factor assuming "good but poorly reconstructed" events have the same probability of passing the acceptance cut as a GoodSingleTrack event.

A multiplicative efficiency correction factor for rejected Single track 2M2S events and Events with multiple track candidates $\bar{\eta}_{T r k)}$ can be defined as follows,

$$
\begin{gathered}
\bar{\eta}_{2 M 2 S}=\frac{N_{2 M 2 S \cap P I D}}{N_{(0 M 4 S \cup 1 M 3 S) \cap P I D}} \\
\bar{\eta}_{M T}=\frac{N_{M T \cap P I D}}{N_{(0 M 4 S \cup 1 M 3 S) \cap P I D}} \\
\bar{\eta}_{T r k}=1+\bar{\eta}_{2 M 2 S}+\bar{\eta}_{M T}
\end{gathered}
$$

$\eta_{T r k}=\frac{1}{\bar{\eta}_{T r k}}$ then can be applied as a regular correction. Table 8 summarizes the multiplicative correction factor for all the kinematics. Efficiency correction for DVCS3 is roughly 6\%-7\%. 


\begin{tabular}{lrrrrrrrr}
\hline \multirow{2}{*}{ Run\# } & \multicolumn{4}{c}{9380} & \multicolumn{4}{c}{9748} \\
& \multicolumn{2}{c}{ Two-Track } & \multicolumn{2}{c}{ Multi-Track } & \multicolumn{2}{c}{ Two-Track } & \multicolumn{2}{c}{ Multi-Track } \\
\hline$E_{\text {min }}$ & Total & Cluster & Total & Cluster & Total & Cluster & Total & Cluster \\
600 & 0.018 & 0.017 & 0.034 & 0.029 & 0.019 & 0.017 & 0.033 & 0.027 \\
700 & 0.016 & 0.015 & 0.027 & 0.023 & 0.017 & 0.015 & 0.027 & 0.022 \\
800 & 0.015 & 0.014 & 0.024 & 0.020 & 0.016 & 0.014 & 0.024 & 0.019 \\
\hline
\end{tabular}

TABLE 7: Correction Factors computed for Two Track events and and all Multi Track candidates events using election selections with total Pion Rejector sum and Pion Rejector Cluster sum. Only higher number of track candidate events shows a large uncertainty in our extracted correction factors, provinding evidence to the idea these events could contain additional low energy electron track

\begin{tabular}{|c|c|c|c|c|c|}
\hline Kin & $\bar{\eta}_{2 M 2 S}$ & $\bar{\eta}_{M T}$ & $\bar{\eta}_{T r k}$ & $\sigma_{\bar{\eta}_{T r k}}$ & $\eta_{T r k}$ \\
\hline 361 & 0.027 & 0.033 & 1.06 & $1.26 \mathrm{E}-03$ & 0.943 \\
\hline 361 & 0.027 & 0.037 & 1.064 & $3.75 \mathrm{E}-04$ & 0.94 \\
\hline 362 & 0.028 & 0.042 & 1.07 & $5.18 \mathrm{E}-04$ & 0.935 \\
\hline 481 & 0.025 & 0.017 & 1.043 & $6.04 \mathrm{E}-04$ & 0.959 \\
\hline 482 & 0.027 & 0.036 & 1.063 & $7.67 \mathrm{E}-04$ & 0.941 \\
\hline 483 & 0.027 & 0.03 & 1.056 & $8.09 \mathrm{E}-04$ & 0.947 \\
\hline 484 & 0.027 & 0.033 & 1.06 & $7.00 \mathrm{E}-04$ & 0.944 \\
\hline 601 & 0.027 & 0.039 & 1.066 & $1.00 \mathrm{E}-03$ & 0.938 \\
\hline 603 & 0.027 & 0.037 & 1.064 & $1.07 \mathrm{E}-03$ & 0.94 \\
\hline
\end{tabular}

TABLE 8: This table summarizes the results for tracking efficiency corrections expressed as a multiplicative correction factor for each kinematics. 


\subsection{ACCEPTANCE STUDIES}

Hall A HRS has angular acceptance of $\pm 30 \mathrm{mr}$ in horizontal and $\pm 60 \mathrm{mr}$ on vertical directions with a momentum acceptance of $\pm 4.5 \%$. In first order approximation, where the trajectory of the particle is determined only by the spectrometer magnetic field, a charged particle entering LHRS, will either propagate to the focal plane detectors, or be blocked by the internal spectrometer apparatus, based on the flight path and particle momentum [73]. This acceptance region can be expressed as a set of R-Functions in 5 dimensional space of $x_{t g}, y_{t g}, \phi_{t g}, \theta_{t g}, \delta_{t g}$, where the first five variable determines the line of flight and the 5 th variable, particle momentum [73].

Rvachev functions or R-Functions, are real valued functions whose sign is determined only by the signs of it's arguments, not by the magnitude. Together with the closed nature under composition, RFunctions constructed to determine the boundary of complex geometries can be used to determine the region of space enclosed by the boundary by checking the sign of the RFunctions. This will also allow to vary the size of the confined region easily, by turning the boundary into an inequality of greater than or less than a certain positive value, rather than zero. This allows a great amount of flexibility in a software based acceptance cut.

Each of the three run periods of DVCS3, posses unique challenges, in terms of Q1 magnet of the spectrometer as discussed in section 4.3.1, that would require different R-Function for different kinematic settings

Two set of RFunctions and RCuts were developed for DVCS3 independently by A. Stefanko [76] and G. Hamad [39]. This work follows the work of A. Stefanko.

Up to 15 sets of $2 \mathrm{D}$ RFunctions are defined in following four $2 \mathrm{D}$ subset of the original $5 \mathrm{D}$ space $(\theta \delta, \phi \delta, \phi y, \theta \phi)$ to fully describe the acceptance space.

$$
\frac{A_{i} x+B_{i} y+C}{\sqrt{A^{2}+B^{2}}}=R_{i}
$$

Here,

$x, y$ makes the plane $y x$ of the $2 \mathrm{D}$ subset and $i$ denotes the $i^{\text {th }}$ RFunction up to a total of up to 15 per kinematic setting. Some Functions are deemed redundant for some kinematics.

$R_{\text {value }}$ for a given event then can be computed as $R_{\text {value }}=\operatorname{Min}\left\{R_{i}\right\} . R_{\text {value }}>$ RCut will form the acceptance cut. Table 9 shows the RCut values defined for different kinematics by comparing the $R_{\text {value }}$ distribution of data to the Monte-Carlo.

Figure 36 shows the effect of R-Function cuts on the 2-D planes they are defined for kinematic setting 603. 


\begin{tabular}{|c|c|}
\hline Kin & RCut Value \\
\hline $36 \_1$ & 0.003 \\
\hline $36 \_2$ & 0.004 \\
\hline $36 \_3$ & 0.004 \\
\hline $48 \_1$ & 0.0045 \\
\hline $48 \_2$ & 0.004 \\
\hline $48 \_3$ & 0.003 \\
\hline $48 \_4$ & -0.002 \\
\hline $60 \_1$ & 0.004 \\
\hline $60 \_3$ & 0.004 \\
\hline
\end{tabular}

TABLE 9: This table summarizes the RCut values as defined by A. Stefanko [75]

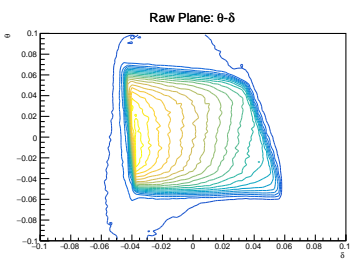

Raw Plane : $\phi-y$

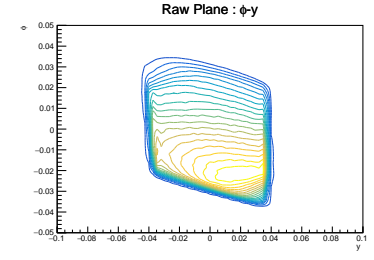

(a) Raw distribution

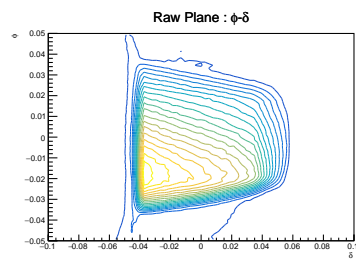

Raw Plane : $:-\phi$

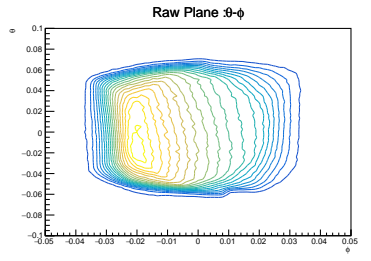

$1 . . .1 .04$

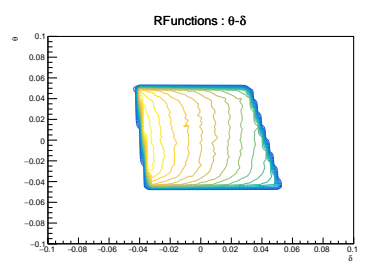

RFunctions : $\phi-y$

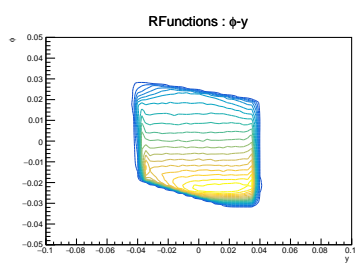

(b) After RFunctions cut
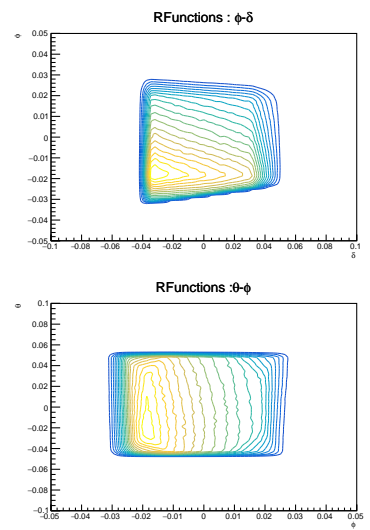

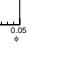




\subsection{TRIGGER EFFICIENCY STUDIES}

In an experiment such as DVCS3 it is important to keep up with the data rates of the DAQ system when recording the data, so it becomes necessary to distinguish potentially interesting events from the background in the DAQ. This is usually achieved by using a trigger system. The DVCS3 experiment looked for events, with a scattered electron (HRS) in coincident with a high energy gamma-ray. Threshold Gas Cherenkov distinguishes between electron and hadron. Similarly DVCS Calorimeter detects the photon. Hence, coincidence of Gas Cherenkov and S2M with DVCS Calorimeter forms the trigger for DVCS production data. The setup of the trigger is described in section 3.5.1.

Inefficiencies in registering the coincidence signal from a good electron in Gas Cherenkov and S2M will result in a Good Event not being recorded in the experimental data. These events should be accounted for. Evaluating the efficiency of the trigger is important to estimate the number of missing events, and usually an important step in a cross-section measurement.

To estimate the trigger efficiencies, in Hall A DVCS experiments, A set of 3 runs each with a different spectrometer trigger is used. Efficiency of a detector trigger is estimated from the run taken without that detector in the trigger, as shown bellow,

- S0กCer Trigger : Evaluate S2M Efficiency

- S0กS2M Trigger : Evaluate Cherenkov Efficiency

- S2กCer Trigger : Evaluate S0 Efficiency

S2M and CER efficiencies are used in cross-section calculations, S0 efficiency is measured as a control variable for experiment.

Trigger efficiency can be defined as, the fraction of good events with a good timing signature of the detector in question among the total recorded good events without the detector in the trigger.

$$
\text { TriggerEfficiency }=\frac{\text { Number of good Electron event with Good Detector Timing }}{\text { Number of Good Electron Event }}
$$

Here, Good Electron Events are determined by

GoodSingleTrack $=$ At Most one Multi Cluster in VDC Chambers

GoodAcceptance $=$ GoodTarget $\cap$ Good RFunctions 
GoodElectronID $_{\text {Trigger }}=$ PionRejectorElectron $\cap$ CherenkovElectron for S0 and S2M efficiency measurement; PionRejector with at least one photo electron in the Gas Cherenkov for Cherenkov efficiency measurement.

Good Detector Timing is defined by analyzing the timing spectrum from each detector. A copy of each timing signal used to form the trigger is sent to DVCS High resolution TDC with 25 pico second time resolution. Gas Cherenkov timing signal, also arrives at LHRS TDC with a 50 pico second time resolution. Figure 37 shows the typical timing spectrum of these different trigger detectors, with the selected timing cut.
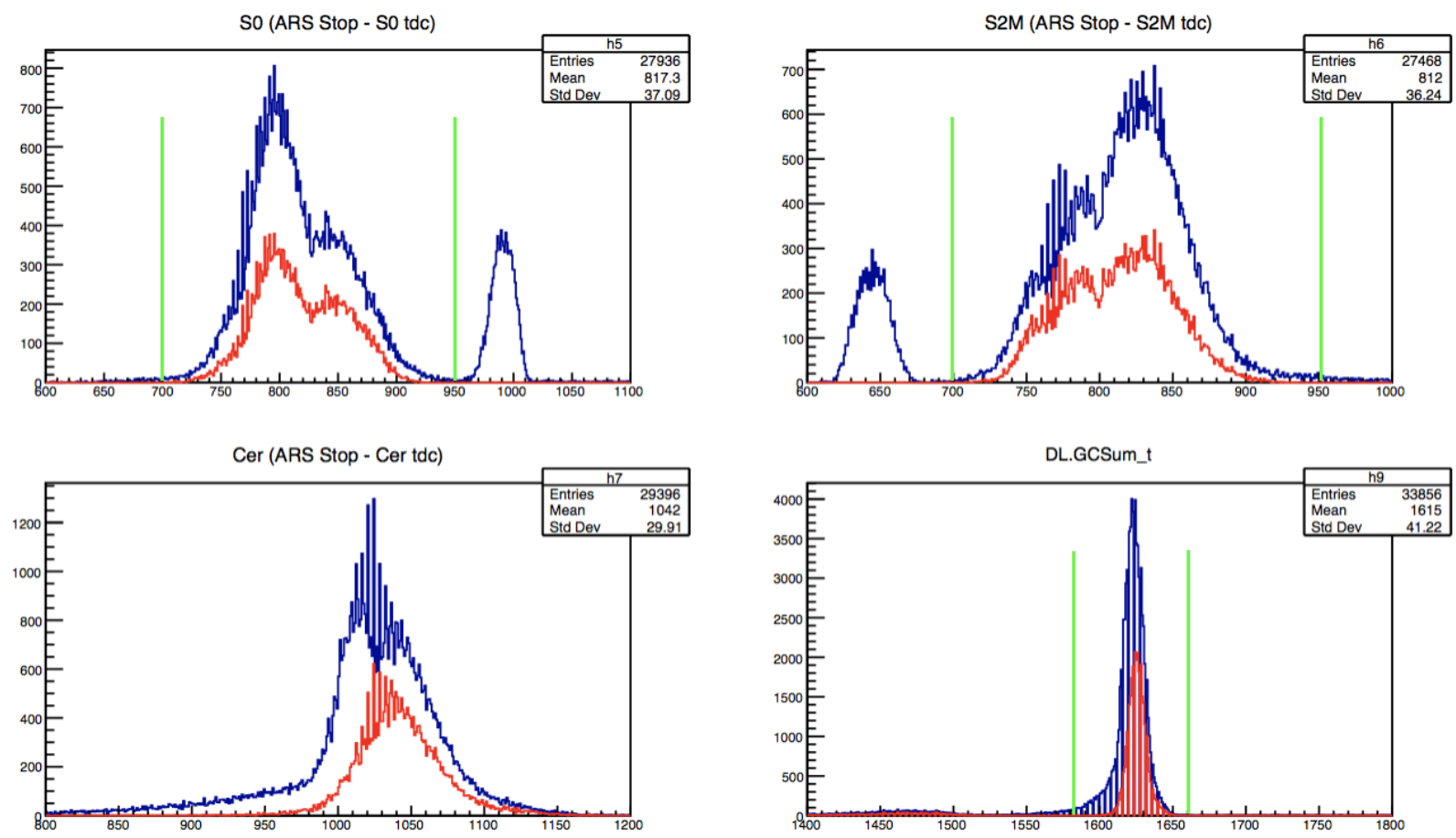

FIG. 37: Typical timing spectra of different triggers. Blue spectrum shows all events and Red spectrum shows Good Single track electron events, with the green lines indicate the cut region for determining good timing signal. Fig 1-3 are from High resolution DVCS TDC and figure 4 is from LHRS TDC

Following is a summary of DVCS3 Efficiency runs

- Fall 2014 : Run spanned 3 days, between December 13 and December 16, 2014. 1 set of efficiency run on December 19.

- Spring 2016 : Feb 13 - April 20. No dedicated efficiency runs. Feb 17, 18 and 19 runs with multiple triggers enabled at once (DVCS, DIS and S0กS2M). 


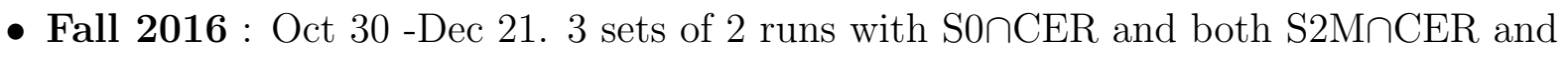
S0กS2M triggers enabled simultaneously on November 2, 4, 6. Dedicated efficiency runs in a 3 day interval from November 24 th.

Fall 2014 and Fall 2016 Detector efficiencies are extracted as mention before. For Spring 2016, the variable triggerPatternWord, which records every coincidence trigger as well as single triggers fired in a recorded event, was used to get the unbiased trigger efficiencies. This method will look for the coincidence triggers without the chosen detector and count the single trigger among them with good timing, with all the previously mentioned event selection cuts, except the once involving the trigger detector in question. S2M detector was always in the trigger in these runs and hence an unbiased trigger efficiency for S2M was not estimated. Instead the efficiency between 2016 Spring and Fall runs were assumed to be similar and the value from Fall data was used for DVCS analysis.

Table 10, 11 and 12 summarize the mean efficiency values for each run period as percentage values.

\begin{tabular}{|c|c|c|}
\hline RunNumber & Detector & Efficiency \\
\hline 10418 & S0 & 99.76 \\
\hline 10415 & S2M & 99.74 \\
\hline 10419 & CER & 99.84 \\
\hline
\end{tabular}

TABLE 10: This table summarizes the trigger efficiency for Fall 2014

\begin{tabular}{|c|c|c|c|}
\hline$N_{\text {iteration }}$ & Detector & Mean.Efficiency & $\sigma$ \\
\hline 17 & S0 & 99.72 & 0.02 \\
\hline 17 & CER & 99.59 & 0.15 \\
\hline
\end{tabular}

TABLE 11: This table summarizes the trigger efficiency for Spring 2016. Spring 2016 has 17 multi trigger runs. S2M detector was always in the trigger in these runs and hence an unbiased trigger efficiency for S2M was not estimated. Instead the efficiency between 2016 Spring and Fall runs were assumed to be similar and the value of $99.6 \%$ is used for DVCS analysis. 


\begin{tabular}{|c|c|c|c|}
\hline$N_{\text {iteration }}$ & Detector & Mean.Efficiency & $\sigma$ \\
\hline 9 & S0 & 99.47 & 0.05 \\
\hline 9 & S2M & 99.62 & 0.08 \\
\hline 9 & CER & 99.77 & 0.09 \\
\hline
\end{tabular}

TABLE 12: This table summarizes the trigger efficiency for Fall 2016. Fall 2016 has 9 dedicated efficiency runs. 


\section{CHAPTER 5}

\section{ELECTRON SELECTION, SPECTROMETER NORMALIZATION AND TRIGGER STUDIES}

This chapter summarizes the electron selection procedure as developed in the previous chapter, and presents the extraction of an absolute $\mathrm{H}\left(e, e^{\prime}\right) p$ cross section in the Bjorken regime of large $Q^{2}$ and large $W^{2}$, also referred to as Deep Inelastic Scattering (DIS).

Extracting DIS cross section and comparing it against the world data serves as a sanity check to establish the performance of the spectrometer electron selection, including the new re-normalization of the Pion Rejector and the tracking efficiency corrections. This should also help to verify the acceptance of the spectrometer and the performance of all the other calibrations.

During the DVCS3 run periods, production events were triggered using the coincidence between Gas Cherenkov and S2 scintillator paddles and validated by FastADC signal in the DVCS calorimeter indicating an electron-photon coincidence. A pre-scaled $S 2 \cap C E R$ autovalidation trigger was also active during the run period recording essentially deep inelastic events. Events recorded by these different triggers were tagged using a triggerPatternWord.

Integrated DIS cross section can be written as follows.

$$
\frac{d^{2} \sigma}{d x_{B} d Q^{2}}=\frac{1}{\eta_{\exp } \times \eta_{R} \times \Gamma} \times \frac{D I S \text { Yield }}{\mathcal{L}}
$$

where,

- $\mathcal{L}$ : Integrated Luminosity

$$
\mathcal{L}=\frac{Q}{e} \times \frac{N_{A} \rho l}{A_{H}}
$$

- Q - Total accumulated charge during a run, this can be calculated run by run

- e - Charge of an electron (1.602E-19 C)

- $N_{A}$ - Avogadro number (6.022E23/mole)

- $\rho$ - Density of the target $\left(0.07229 \mathrm{~g} / \mathrm{cm}^{3}\right.$ at the operating pressure $(25 \mathrm{psi})$ and temperature $(17 \mathrm{~K})$ of the target) 
- $l$ - Length of the hydrogen target $(15 \mathrm{~cm}$, minus the region excluded by the target vertex cut)

- $A_{H}$ - Atomic Mass of hydrogen $(1.0079 \mathrm{~g} / \mathrm{mole})$

- $\Gamma$ - The effective phase-space containing all accepted events. This is computed by using a Monte-Carlo event generator.

- $\eta_{R}$ - Radiative Correction

- $\eta_{\text {exp }}$ - Experimental Corrections - This is a correction for the lost events due to any inefficiency in the yield extraction procedure, including DAQ dead time and tracking efficiency correction.

- DIS Yield - Number of DIS events accepted by the event selection algorithm.

\subsection{DIS YIELD EXTRACTION}

We apply following event section cuts to select a good electron in the spectrometer developed in Chapter 4

- Electron ID (Section 4.2.2) :

- Cherenkov Electron : Events producing more than 1.5 photo electron

- Pion Rejector Electron : Any electron producing more than $60 \%$ of the full energy electron peak with more than $20 \%$ on the first layer of the Pion Rejector is taken to be a good Pion Rejectror electron

- Good Single Track (Section 4.4.3): At most one multi cluster in Vertical Drift Chamber wire planes.

- Target Vertex (Section 4.3.2): Events produced within the central $13 \mathrm{~cm}$ of the Liquid Hydrogen target in $\mathrm{Z}$ direction.

- Acceptance (Section 4.5): A set of up to 15 RFunctions in 4 different planes in the 5 dimensional space of $x_{t g}, y_{t g}, \phi_{t g}, \theta_{t g}, \delta_{t g}$.

In addition to the above cuts, DIS triggered events can be selected by checking for the bit 0x00080 in triggerPatternWord. DIS Yield, then would be the number of events passing all 
the above cuts multiplied by the DIS pre-scale. Unfortunately, this is the most troublesome part of the entire exercise and discussed in detail in section 5.2 .

\subsection{DIS TRIGGERS AND PRE-SCALE AMBIGUITY}

For certain run periods, pre-scaled S0 $\cap$ Cer coincidence and/or S0 $\mathrm{S} 2$ coincidence trigger was/were also active in addition to the $S 2 \cap C E R$ calo-validation and pre-scaled $S 2 \cap C E R$ auto-validation triggers, recording additional events in order to evaluate efficiencies of S2 and Gas Cherenkov detectors as triggers as stated in section 4.6. S0 is a single large scintillator paddle and $\mathrm{S} 2$ is a group of 16 scintillator paddles, discussed in section 3.3 .2

The DVCS DAQ received logic pulses (analog in time) from the standard HRS electronics, indicating S0, Cherenkov, and S2M signals. The DVCS DAQ is based on Field Programmable Gate Array (FPGA) hardware and logic programming: the leading and trailing edges of incoming logic pulses are locked to the next occurring clock pulse ( $5 \mathrm{~ns}$ timing). The FPGA code then formed coincidences, applied pre-scale factors and determined which events were recordable (up to live-time limitations) discussed in section 3.5.1. A bit pattern word in the FPGA recorded all of the valid trigger combinations of each of these coincidences in a recorded event. This triggerPatternWord variable was then used in the analysis to separate events from each individual trigger for appropriate scaling. However, the early arrival of a particular trigger could cause other trigger signals to lie outside the latch window of the FPGA, causing valid trigger signals to not be recorded in the Bit Pattern Word.

\subsubsection{MIS-LABELLED EVENTS}

In our Spring 2016 data, when $S 0 \cap C E R$ coincidence was in the trigger, we had a considerable portion of good electron events in the data stream with good $S 2$ and $C E R$ signals but with no $S 2 \cap C E R$ coincidence tag in the triggerPatternWord variable and hence escaping the trigger selection cut for the DIS. These events are mostly confined to the low $\delta P$ region. Figure 38 shows the $\delta P$ spectrum of all the events having a trigger tag corresponding to DIS events for two of the Spring 2016 runs, one with $S 0 \cap C E R$ in the trigger (12541) and one without. (12518). We observe missing events in the low $\delta P$ region. DVCS trigger was set up in a way that S2 timing always sets up the coincidence window. S0 is a single long scintillator paddle placed vertically in the lab frame, essentially along the $\delta p$ axis. Each end of the paddle is attached to its own photo tube. However, only the phototube from one end of the scintillator is feeding the S0 timing and thus creating a huge spread in S0 timing between high $\delta P$ vs low $\delta P$ events shown in Figure 39 . This could cause the trigger 

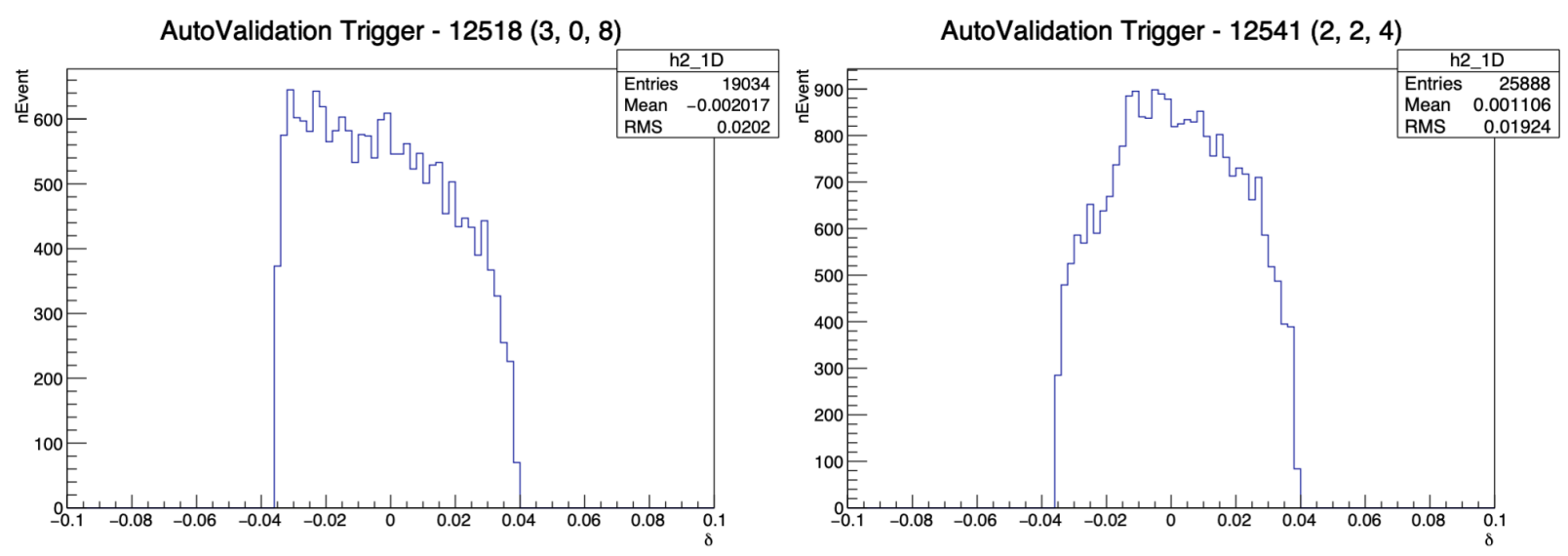

FIG. 38: On the left, $\frac{\delta P}{P}$ spectrum for Kin 481 run 12518 without $S 0 \cap C E R$ in the trigger. On the right $\delta p$ spectrum for same kinematic run 12541 with $S 0 \cap C E R$ in the trigger. Shape of the spectrum suggests that there are events missing in the low $\delta p$ region.

supervisor to lose the trigger tag for $S 2 \cap C E R$ in the presence of $S 0 \cap C E R$ trigger for some events in the low $\delta P$ region.

Figure 40 shows the presence of $S 2 \cap C E R$ coincidence peaks in the $S 0 \cap C E R$ triggered events without the trigger tag for $S 2 \cap C E R$. These events are in fact recoverable by including $S 0 \cap C E R$ trigger - which is also a valid DIS trigger - in the trigger selection. The ambiguity however is on the correct pre-scale factor to be used for these events.

In the ideal condition of no $\mathrm{S} 0$ interference in determining the $S 2 \cap C E R$ coincidence, all of these events should be subjected to the pre-scale on $S 0 \cap C E R$ trigger since they would only have the $S 0 \cap C E R$ coincidence and are the events normally be attributed to $\mathrm{S} 2$ trigger inefficiency. Unfortunately, it is not that simple here since these events satisfies the trigger condition for both coincidence triggers, and knowing the correct pre-scale factor to multiply is near impossible without knowing exactly which trigger recorded which event. For this reason in the previous normalization studies, when good electron events were found without the trigger tag for DIS trigger, they were simply added without any pre-scale.

In reality, at least in the first order approximation the total number of events would be anywhere between Number of Exclusive $S 0 \cap C E R$ events and Number of Exclusive $S 0 \cap C E R$ events $\times$ Largest of the two Pre-Scales. I will attempt to minimize this range of error caused by pre-scale ambiguity as much as possible by using the CaloTriggered variable to distinguish the state of calo-validation (validation by the Calorimeter Fast ADC) in addition to the triggerPatternWord and establish a new method of yield extraction. 

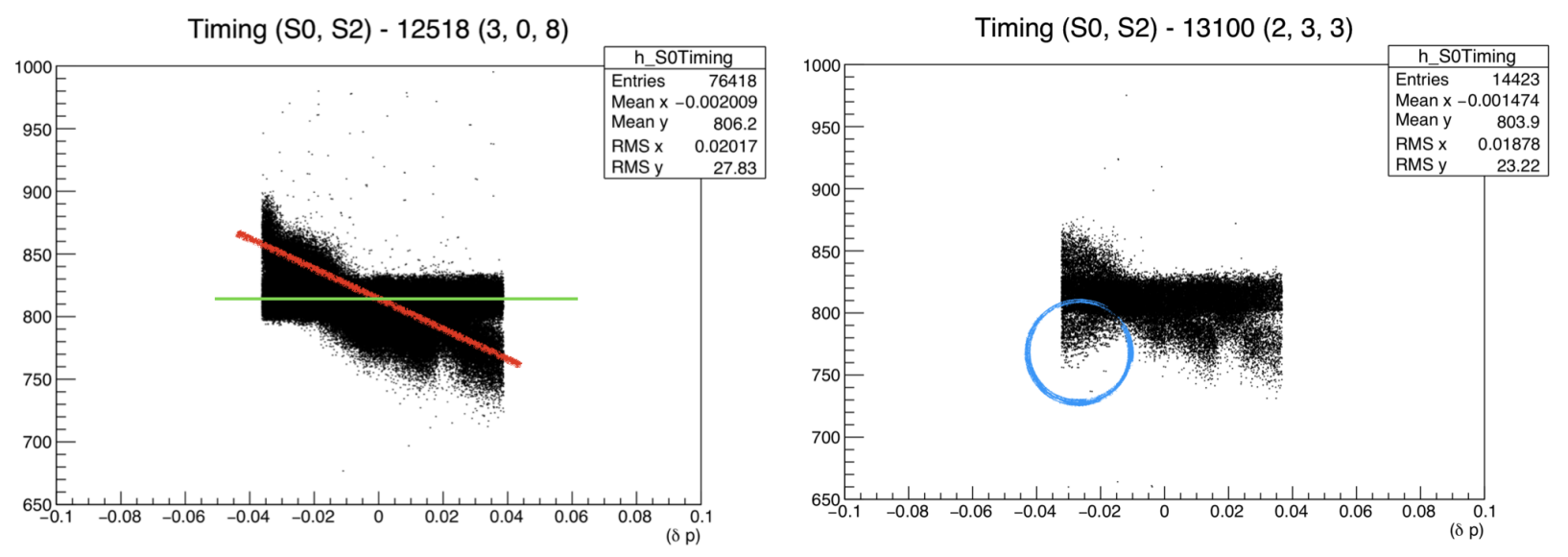

FIG. 39: Plots show overlaid S0-ARSStop and S2-ARSStop timing spectra (time on the vertical axis) as functions of the reconstructed tract $\delta p / p$ value on the horizontal axis, for two of the spring 2016 runs. The spread following the red line shows the S0 timing and green line is S2 timing. S0 spectrum shows about a 50 ns spread. Plot on the left is for Run 12518 without $S 0 \cap C E R$ in the trigger. Plot on the right for run number 13100 shows an additional set of events with an unexpected S0 timing in the presence of $S 0 \cap C E R$ trigger in low $\mathrm{x}$ region. The numbers in parentheses next to the run number in the plot titles are the pre-scale factor for DIS, $S 0 \cap C E R$ and $S 0 \cap S 2$ triggers in that order

S2_CER Timing - 13150 (DIS- 2, SOCER-2)

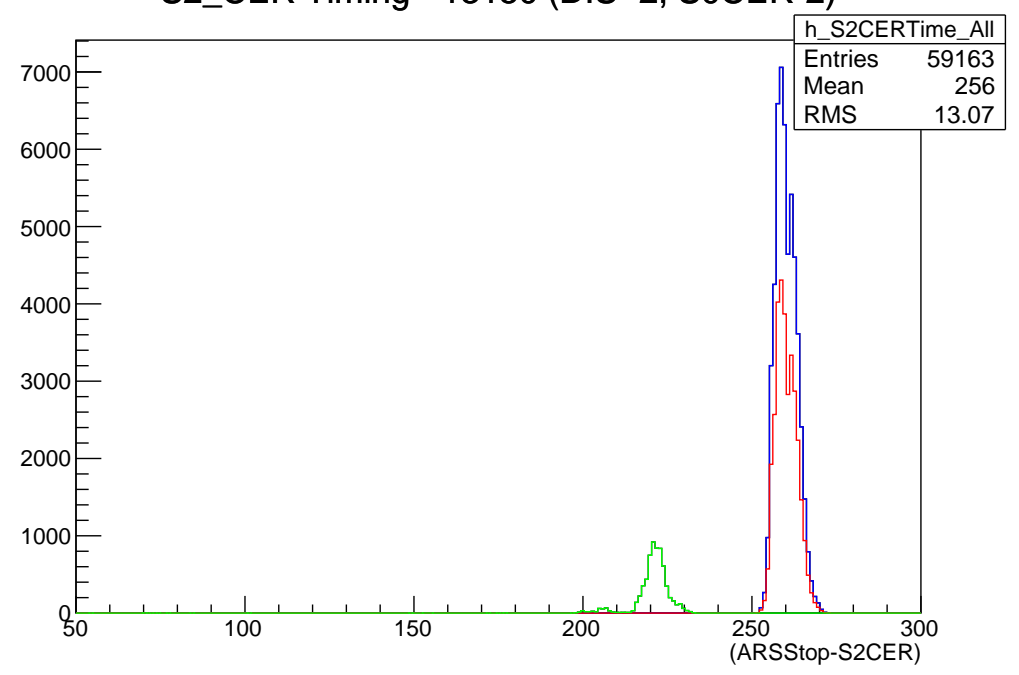

FIG. 40: Plot shows $S 2 \cap C E R$ coincidence time with respect to ARSStop separated by triggerPatterWord tags. Blue spectrum is Inclusive DVCS, Red is Inclusive DIS and Green in Exclusive $S 0 \cap C E R$. Events from $S 0 \cap C E R$ coincidence trigger shows $S 2 \cap C E R$ coincidence time peaks, even though they were not tagged. (Here, Exclusive means events with only the spoken trigger tag and Inclusive means events with spoken trigger tag with other additional trigger tags) 


\subsubsection{RESOLVING THE PRE-SCALE AMBIGUITY}

Let's consider two triggers, trigger $\mathrm{A}$ and trigger $\mathrm{B}$ with pre-scale $\mathrm{a}$ and $\mathrm{b}$ (with $b>a$ ) recording events in tandem and $\mathrm{N}$ number of events satisfying both trigger conditions. Say, the trigger system recorded $N_{R}$ number of events in total. If the pre-scales on both triggers are in lock steps, Trigger A would record $\frac{N}{a}$ number of events and trigger B would have recorded $\frac{N}{b}$ number of events. In this scenario, the trigger system would have recorded exactly $\frac{N}{a}$ number of events and every events recorded by trigger B would be a subset of events recorded by trigger $\mathrm{A}$. This would be the same for a three trigger system. Total number of recorded events will be that of the trigger with lowest pre-scale. If the pre-scale are out of sync, trigger A still would have recorded $\frac{N}{a}$ and Trigger B $\frac{N}{b}$, but now the recorded event pool could contain mutually exclusive event set. Pre-scale can get out of sync due to a particle only triggering one trigger vs the other due to some inefficiency, when this happen again, the pre-scale sync will restored. This could happen many times during the cause of a run. In the extreme end, the trigger system could have recorded a total of $\frac{N}{a}+\frac{N}{b}$ events, making $N_{R} \geq \frac{N}{a}$. For this trigger system we can derive, $N \leq a \times N_{R}$. If one of these trigger is set to record all the events, there is no potential of recording additional events, $N=N_{R}$, no scaling is necessary.

In our case of three trigger system and S0 interference, the exclusive SOกCER events could be of following sub categories,

- A DVCS triggered event bumped by S0 timing: These events are all calo-validated and should be scaled 1 .

- A DIS Triggered event bumped by S0 timing. These events are auto-validated and should be scaled by DIS pre-scale

- Events recorded by the $S 0 \cap C E R$ trigger in addition to other two triggers: This would include regular $\mathrm{S} 2$ inefficiency and are auto validated and should be scaled by $S 0 \cap C E R$ pre-scale.

If we separate the calo-validated portion from the exclusive $S 0 \cap C E R$ events, those wouldn't require scaling. This portion is the only one matters for DVCS analysis. Looking for a good electron with a DVCS photon in coincidence will automatically recover these events. In DIS analysis on the other hand, we need to recover the portion of events without a calo-validation. Only DIS and $S 0 \cap C E R$ trigger would have recorded the events without a 
calo-validation. In this case, the straight forward assumption would be to scale these events by the lowest pre-scale of the two triggers.

Separating out these event by calo-validation(Calo) and no calo-validation (!Calo) would enable us to minimize the error introduced by pre-scale ambiguity. A) By using the fact that DVCS trigger suppose to record every event of that category and B) By breaking the three trigger problem in to 2 at a time

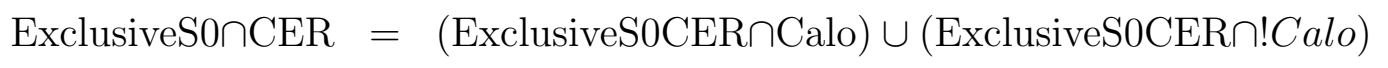

$$
\begin{aligned}
& \text { Yield }_{\text {Mis_Labelled }}=(\text { ExclusiveS0CER } \cap \text { Calo }) \\
& +\min \left\{\text { Scale }_{D I S}, \text { Scale }_{S 0 C E R}\right\} \times(\text { ExclusiveS0CER } \cap \text { Calo })
\end{aligned}
$$

Unfortunately, things are a little bit tricky here in the !Calo part of the event pool. But luckily, the solution is very simple. DVCS3 trigger setup by default calo-validate all HRS coincidences. The trigger bits sent for auto-validation were set separately in the trigger configuration file [78]. A bit value was posted to the start of run log entries denoting the triggers sent for auto-validation [29]. DIS trigger is set as a proper $S 2 \cap C E R$ timing to go through autovalidation. Since the trigger supervisor sees the exclusive $S 0 \cap C E R$ events as only passing the $S 0 \cap C E R$ trigger, they would not get auto validated according to the $S 2 \cap C E R$ pre-scale rather only as $S 0 \cap C E R$ triggered events, hence governed by it's prescale. That would imply exclusive $S 0 C E R \cap ! C a l o$ events should be scaled by $S_{c a l e}$ SonCER . This will give us following formulae for yield correction.

$$
\begin{aligned}
\text { Adjusted DIS Yield }= & \#{\text { Exclusive SOCER Calo }+ \text { Scale }_{D I S} \times \# D I S} \\
& + \text { Scale }_{S 0 C E R} \times \# \text { Exclusive SOCER } \cap \text { Calo }
\end{aligned}
$$

We could also see that,

$$
\#(D V C S \cap \text { Calo })=\text { Scale }_{D I S} \times \#(\text { DIS Calo })
$$

by using the Equation 66, we can arrive at the following formula for yield correction.

$$
\begin{aligned}
\text { Adjusted DISYield } & =\# \text { GoodElectron } \cap \text { Calo }+ \text { Scale }_{D I S} \times(\# \text { DIS } \cap \text { !Calo }) \\
& + \text { Scale }_{S 0 C E R} \times \# \text { Exclusive S0CER } \cap \text { Calo }
\end{aligned}
$$

Figure 41 shows an application of equation 67 to recover the $\delta p$ spectrum. A more natural shape on the adjusted spectrum verifies the validity of equation 67 for DIS yield correction. Inclusion of Exclusive SOCER Calo in the yield would recover all the missed events due to 
the regular inefficiency of S2 in addition to the amplified inefficiency caused by S0 timing. In this case the regular S2 inefficiency correction discussed in section 5.4.3 should be be omitted for the adjusted DIS Yield.

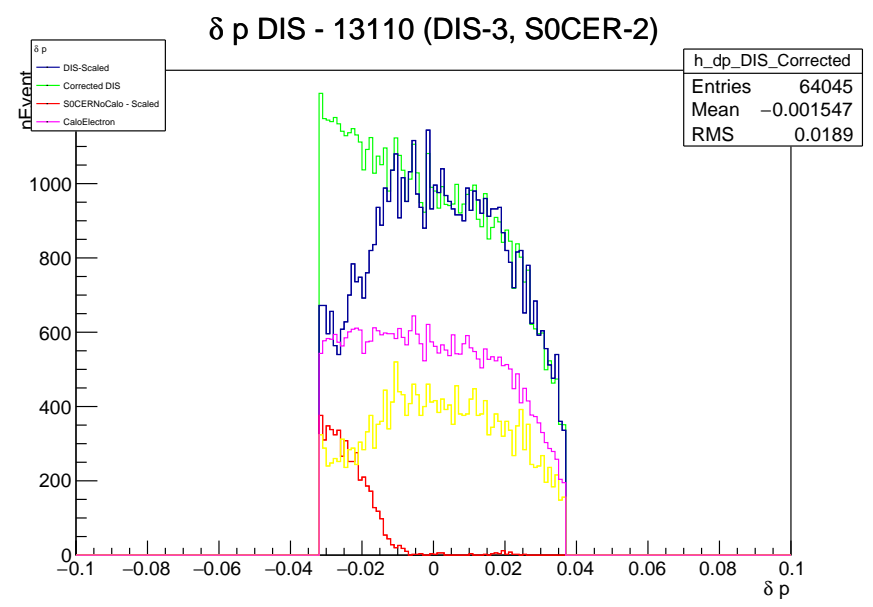

FIG. 41: Plot shows yield corrected $\delta p$ spectrum for Run 13110 using equation 67. Blue spectrum is scaled DIS (uncorrected). Green is the corrected spectrum. Magenta is

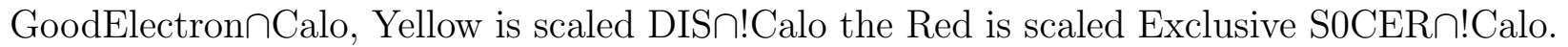
Green spectrum is the sum of Red, Yellow and Magenta and recovers a more natural shape for $\delta p$ distribution.

\subsection{CHARGE CALCULATION}

Hall A provides a system of Unser monitor and RF Cavities for beam current monitoring, discussed in section 3.3.1. Scaler signals from the RF Cavities are calibrated against the Unser monitor during calibration runs. In a calibration run, current is ramped up from zero to the max current in cycles to make sure the drift of the Unser monitor is taken into account. Scaler outputs from the coda run taken at the same time is then calibrated together with the Unser monitor. These calibrations are taken every 2-3 months and are stable up to $\pm 0.5 \%$ [5]. The Unser Calibration for DVCS3 runs were performed by J. Roche and B. Karki [52. The calibration constant found for each run period is then used in charge calculations. Among the multiple BCM scalers, scaler D3 was found to be the most stable and used for charge calculations for DVCS3 runs. Table 13 gives the calibration coefficient for D3. 


\begin{tabular}{|c|c|c|c|}
\hline Run Period & Kinematic settings & Gain & Offset \\
\hline Fall 2014 & 36_1 & $1.274 \mathrm{E}-4$ & 0.39 \\
\hline Spring 2016 & $48 \_1,48 \_2,48 \_3$ and 48_4 & $9.309 \mathrm{E}-5$ & 0.30 \\
\hline Fall 2016 & 36_2, 36_3, 60_1 and 60_2 & $9.705 \mathrm{E}-5$ & 0.19 \\
\hline
\end{tabular}

TABLE 13: This table summarizes the BCM calibration Coefficient from B. Karki [50]

\subsection{EXPERIMENTAL EFFICIENCY CORRECTIONS}

Table 14 provides a summary of all the corrections factors used in the normalization studies for each kinematics. Tracking and Dead-time corrections are applied run by run and the trigger efficiency correction is determined for the whole run period. Subsequent sections will discuss the correction factors in details.

\subsubsection{DAQ DEAD TIME}

Data Acquisition System (DAQ) discussed in section 3.5 shows that some real events can be lost during the time the system is procession an event. This time in which the DAQ system is not available to record new data is called the DAQ dead-time. A dead-time correction factor should be established in order to accounting for this lost events. A scaler system, which only record the count of events rather than the events themselves individually with all the information can avoid the huge delay in the processing and hence can be used to measure the dead-time correction.

We use two scalers to estimate dead time correction factor for each trigger used in DVCS3. One scalar counts every time there is a coincidence in the trigger regardless of whether it is recorded by the DAQ or not. We call it the Raw Scalar. Another scalar increments only when there is a coincidence and the DAQ is not busy, we call it the Live scalar.

Dead-time correction factor, then can be defined as

$$
\eta_{D T}=\frac{\text { Live Scalar }}{\text { Raw Scalar }}
$$

It is computed run-by-run.

\subsubsection{TRACKING EFFICIENCY CORRECTION}

Only events with at most one multi cluster in the VDC wire planes were selected for the yield as discussed in section 5.1. Tracking Efficiency Correction, accounts for the events 
excluded from the analysis as discussed in section 4.4.6. For the normalization studies I computed the tracking corrections run-by-run.

\subsubsection{TRIGGER EFFICIENCY CORRECTION}

DIS events are triggered using the coincidence between S2 and Gas Cherenkov. Any inefficiency in either detector in producing a proper time signal would have caused the trigger to miss events. We calculate the efficiency of a given detector, using events triggered by the coincidences of the other detector with S0 from dedicated efficiency runs as described in section 4.6

\begin{tabular}{|c|c|c|c|c|}
\hline Kin & $\eta_{\text {Tracking }}$ & $\eta_{\text {Trigger_S2 }}$ & $\eta_{\text {Trigger_CER }}$ & $\eta_{\text {Livetime }}^{\text {DIS }}$ \\
\hline $36 \_1$ & 0.943 & 0.997 & 0.998 & 0.981 \\
\hline $36 \_2$ & 0.940 & 0.996 & 0.998 & 0.980 \\
\hline $36 \_3$ & 0.935 & 0.996 & 0.998 & 0.966 \\
\hline $48 \_1$ & 0.959 & - & 0.996 & 0.985 \\
\hline $48 \_2$ & 0.941 & - & 0.996 & 0.963 \\
\hline $48 \_3$ & 0.947 & - & 0.996 & 0.985 \\
\hline $48 \_4$ & 0.944 & 0.996 & 0.996 & 0.978 \\
\hline $60 \_1$ & 0.938 & 0.996 & 0.998 & 0.979 \\
\hline $60 \_3$ & 0.940 & 0.996 & 0.998 & 0.974 \\
\hline
\end{tabular}

TABLE 14: This table shows experimental correction factors.

\subsection{PHASE-SPACE AND RADIATIVE CORRECTION}

$$
\Gamma=\frac{N_{D e t}}{N_{G e n}} \times \Gamma_{M C}
$$

An event generator which generates events uniformly in a given $\left[\chi_{B}, Q^{2}, \phi_{e}\right]$ space can be used to compute the Phase-Space from which the accepted DIS events are coming. The spacespace is limited by the acceptance, but radiative effects can migrate an event originated outside of the acceptance region in to the acceptance region thus the phase-space can be large and a proper accounting for these effects is needed.

Since the phase-space of the events at the vertex can be much larger than that at the detector, the kinematics at which the cross section is extracted could vary from the kinematics 
at the detector, and to correct for this, a quantity $\alpha$ is defined as follows

$$
\alpha=\frac{1}{\Gamma \times\left.\frac{d \sigma}{d \Omega d E}\right|_{\text {at detector Kin }}} \sum_{\text {accepted events }} \Gamma_{M C}^{i} \times\left.\frac{d \sigma}{d \Omega d E}\right|_{\text {at vertex kin }}
$$

the quantity $\alpha$ here requires a parametrization of DIS cross section suitable to use at the kinematics of the DVCS3.

\subsubsection{EXTERNAL AND INTERNAL RADIATIVE CORRECTION}

\section{External Radiative Correction}

The radiative loss due to the incoming electron traveling through the target material before scattering and the scattered electron traveling through the target before exiting the target cell could be accounted by applying a Bremsstrahlung correction of the form

$$
\frac{\Delta E}{E}=a^{\frac{1}{b t}}
$$

with $a \in[0,1]$ a uniform random number, $b \simeq 4 / 3$, and $t$ is the material thickness in radiation length through which the electron travelled. This generates an electron spectrum following the general expression estimated in [63],

$$
I\left(E_{0}, \Delta E, t\right)=\frac{b t}{\Delta E}\left[\frac{\Delta E}{E_{0}}\right]^{b t}
$$

\section{Internal radiative correction}

The internal radiative correction, where the radiation occurs at the vertex, whether a virtual photon exchange between the incoming and the scattering electron $\left(\delta_{\text {vertex }}\right)$, or a vacuum fluctuation of the scattered electron $\left(\delta_{\text {vacc }}\right)$ or an Internal Bremsstrahlung, where either the incoming or the scattered electron emitting a real photon $\left(\delta_{\mathrm{R}}\right)$ would yield a modification to the extracted cross section.

$$
\sigma_{\text {measured }}=\sigma_{\text {real }} \times \eta_{R}
$$

$\eta_{R}$ can be defined as follows [79]

$$
\eta_{R}=\frac{e^{\delta_{R}^{0}+\delta_{\text {vertex }}}}{\left(1-\delta_{\text {vacc }}\right)^{2}}
$$

where $\delta_{i}$ are $\mathrm{f}\left(Q^{2}, \theta_{e}\right)$. 
Initial normalization study with DIS cross section extraction was carried out by A. Stefanko and B. Karki independently, using the DIS parametrization developed by Eric Christy et al. [21]. During their studies they found that the extracted cross section differed from the world data by up to $9 \%$ in some kinematics [51]. The bigger discrepancy was coming from fall 2016 data, where we had a known optics issue. We also had a known issue with the trigger tagging mechanism that brought some pre-scale ambiguity and complexities in the yield extraction discussed in 5.2. I developed this study to understand this discrepancy and it's effects on actual DVCS analysis and hence I use the phase-space and radiative correction factors computed by A. Stefanko [74] and B. Karki [53] listed in Table 15. B.Karki provides two different computation for the factors, using two different simulations. One using a modified version of standard DVCS simulation adapted for the DIS studies by eliminating the photon generation and calorimeter simulation and other Simulation provided by Eric Christy adapted to the new hall A optics. A. Stefanko uses her own DIS simulation.

\begin{tabular}{|c|c|c|c|c|c|c|c|c|}
\hline Kin & $\alpha_{A}$ & $\Gamma_{A}\left[\mathrm{GeV}^{2}\right]$ & $\eta_{\mathbf{R}}^{\mathrm{AB}}$ & $\alpha_{B}$ & $\Gamma_{B}\left[\mathrm{GeV}^{2}\right]$ & $\alpha_{E}$ & $\Gamma_{E}\left[\mathrm{GeV}^{2}\right]$ & $\eta_{\mathbf{R}}^{\mathbf{E}}$ \\
\hline $36 \_1$ & 0.948 & $0.503 \mathrm{E}-3$ & 1.077 & 0.8633 & $5.229 \mathrm{E}-4$ & 1.03919 & $4.56195 \mathrm{E}-4$ & 1.0511 \\
\hline $36 \_2$ & 0.867 & $0.726 \mathrm{E}-3$ & 1.078 & 0.865 & $6.962 \mathrm{E}-4$ & 1.05158 & $6.076 \mathrm{E}-4$ & 1.041 \\
\hline $36 \_3$ & 0.862 & $1.143 \mathrm{E}-3$ & 1.079 & 0.863 & $11.066 \mathrm{E}-4$ & 1.053 & $9.6701 \mathrm{E}-4$ & 1.036 \\
\hline $48 \_1$ & 0.946 & $0.116 \mathrm{E}-3$ & 1.076 & 0.947 & $1.1365 \mathrm{E}-4$ & 1.05635 & $1.1036 \mathrm{E}-4$ & 1.0387 \\
\hline $48 \_2$ & 1.226 & $0.508 \mathrm{E}-3$ & 1.079 & 1.209 & $5.095 \mathrm{E}-4$ & - & - & - \\
\hline $48 \_3$ & 1.057 & $0.422 \mathrm{E}-3$ & 1.080 & 1.0371 & $4.133 \mathrm{E}-4$ & - & - & - \\
\hline $48 \_4$ & 1.133 & $0.443 \mathrm{E}-3$ & 1.082 & 1.123 & $4.387 \mathrm{E}-4$ & - & - & - \\
\hline $60 \_1$ & 0.914 & $1.405 \mathrm{E}-3$ & 1.080 & 0.885 & $13.8025 \mathrm{E}-4$ & 1.09753 & $13.8585 \mathrm{E}-4$ & 0.911 \\
\hline $60 \_3$ & 0.920 & $0.904 \mathrm{E}-3$ & 1.083 & 0.889 & $8.868 \mathrm{E}-4$ & 1.08217 & $8.678 \mathrm{E}-4$ & 0.943023 \\
\hline
\end{tabular}

TABLE 15: This table shows the Phase-Space and Radiative Correction factors from A. Stefanko [74, and B. Karki 53. Subscript or Superscript B and E denotes the computations from Bishnu Karki with two different simulations. A- denotes the computation by Alexa Stefanko with a $3_{r d}$ simulation. All three computations are using the same DIS parametrization from [21]

\subsection{DIS CROSS SECTION}

As mentioned before in section 5.5, the purpose of this study is to understand the discrepancies in the previous normalization studies, and I use the radiative correction and phase-space factors from A. Stefanko [74], and B. Karki [53]. Since all the event selection cuts are standardized between all analysis, there should not be any discrepancy in the computed 
phase-space and the accepted space-space in the yield analysis. However to account for this fact, and to keep consistency, lets modify equation 61 as follows, and call it Fancy Normalized DIS Yield rather than a Cross-Section.

$$
Y_{\text {fn }}=\frac{D I S Y \text { ield }}{\mathcal{L} \eta_{\text {exp }}} \times \frac{1}{\eta_{R}^{j} \alpha_{i} \Gamma_{i}}
$$

where, $i \in\{\mathrm{A}, \mathrm{B}, \mathrm{E}\}$ and $j \in\{\mathrm{AB}, \mathrm{E}\}$ are from the table Table 15

Figure 42 shows run by run cross section for kinematic 603 using three different model DIS cross-section normalization. The cross section extraction shows good agreement run-by run within each method, and the variation between the methods suggest that it is mostly due to the uncertainties in normalizing the model DIS cross-section rather than any overarching acceptance issue. Figure 43 shows the run-by-run cross section for kin 483, showing the improvement made by the new yield extraction method. The pre-scale ambiguity amounts for about $9 \%$ variation in this kinematic. First look would suggests that the run over run stability of cross section was better in the previous extraction vs the new extraction, a closer look suggests that the variation is coming due to the calorimeter cluster threshold set for the triggering cluster analysis discussed in section sec.caloThresh, since the new yield analysis utilizes the variable caloTriggered which is dependent on the set software threshold.

\subsection{CONCLUSIONS}

Table 16 shows the DIS Cross-Section extracted using equation 75 for the cross-section and 67 for the yield extraction of Spring 2016 kinematics, with the results from the two previous studies by A. Stefanko [74], and B. Karki [51]. All cross sections are expressed as a fraction $\frac{\sigma_{i}}{\sigma_{s}}$ where $\sigma_{i} \in(\mathrm{A}, \mathrm{B}, \mathrm{E})$ and $\sigma_{s}$ is the reference cross-sectionThe new yield extraction method derived in Equation 67 drastically improved the cross section extraction for Spring 2016 kinematics. After the new extraction, the average variation over all kinematics came down to $1 \%$ compared to the world data from the previous $4 \%$ while the variation between the three methods of cross-section normalization remained the same. This indicates the variation in DIS cross-section observed in the previous studies are mostly due to the prescale ambiguity and uncertainties in the model cross-section normalization rather than the acceptance. A more careful analysis As discussed in section 5.2.2, since the Calo-validated $S 2 \cap C E R$ trigger known as the DVCS trigger was always pre-scaled 1, the DVCS analysis does not require any selection on the triggerPatternWord and is immune from any pre-scale ambiguity discussed in here. 


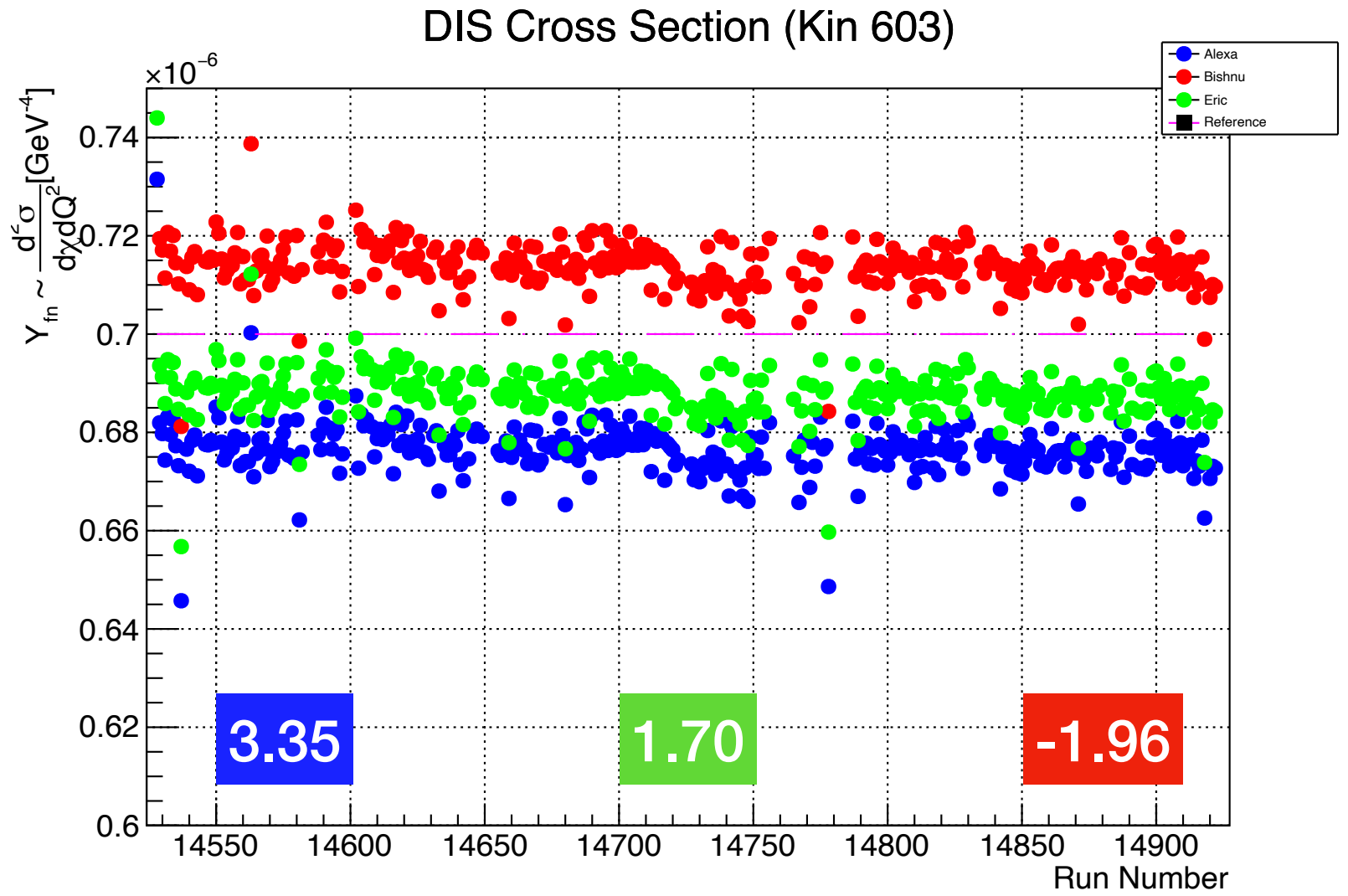

FIG. 42: Plot shows the run-by-run extraction of DIS cross section for Kin 603 using equation 75. Red (Bishnu) Blue(Alexa) and Green (Eric) are using 3 different $\alpha$, Гand $\eta_{R}$ given in Table 15, which were computed with the same cross-section parametrization but with 3 different simulations. All extraction of the cross section uses extracted Yield with the same acceptance cuts, implying the variations among them are due to the uncertainties of normalizing the model cross section rather than the uncertainty in the acceptance. The numbers stated are the average percentage variations of each extraction from the reference cross section for the kinematics. 


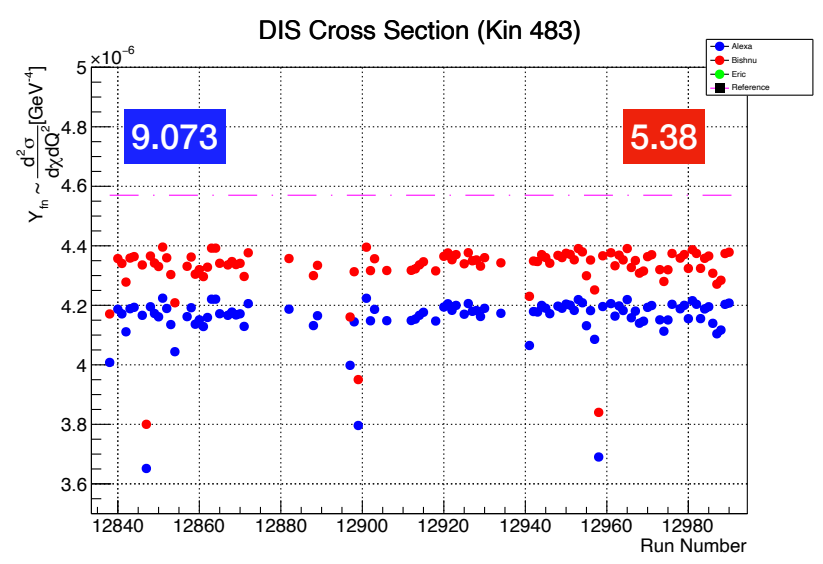

(a) Old Yield extraction method.

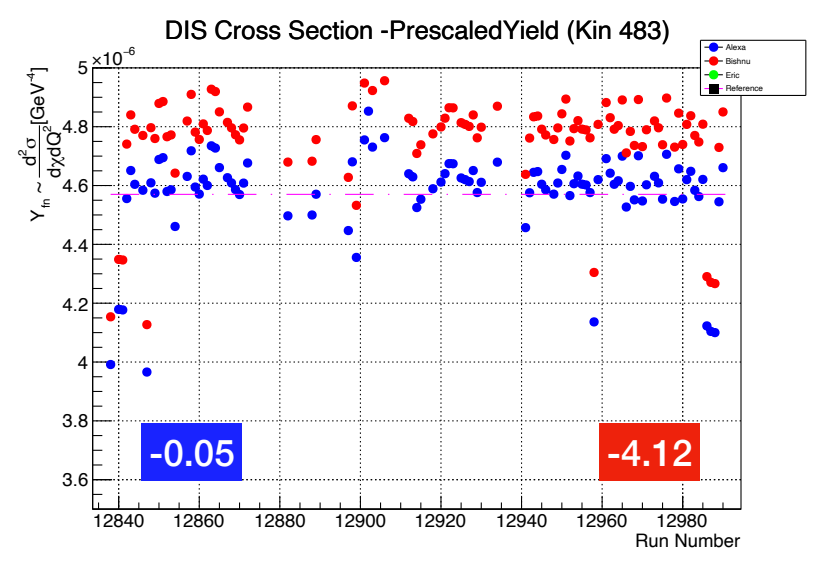

(b) New Yield extraction method.

FIG. 43: Figure shows the run-by run DIS cross section for Kin 483, a spring 2016 kinematics. Figure (a) shows the cross section using the old yield extraction and Figure (b) shows the cross section using the new yield extraction, indicating the improvement made by the Equation 67 in Yield extraction. Red (Bishnu) and Blue(Alexa) are using 2 different $\alpha$, Гand $\eta_{R}$ given in Table 15 , which were computed with the same cross-section parametrization but with 2 different simulations. All extraction of the cross section uses extracted Yield with the same acceptance cuts. The simulation labelled Eric was not available for this kinematics. The numbers stated are the average percentage variations of each extraction from the reference cross section for the kinematics, negative sign implying the data is above the reference 


\begin{tabular}{|c|c|c|c|c|c|c|c||c|c|}
\hline Kin & $\left.\frac{d^{2} \sigma}{d x d Q^{2}}\right|_{s} G e V^{4}$ & $A_{\text {old }}$ & $B_{\text {old }}$ & $E_{\text {old }}$ & $A_{\text {new }}$ & $B_{\text {new }}$ & $E_{\text {new }}$ & $A v . A g_{\text {old }}$ & $A v . A g_{\text {new }}$ \\
\hline $36 \_1$ & $2.798 \mathrm{E}-5$ & 0.99 & 0.95 & 0.97 & 0.99 & 0.93 & 0.96 & 0.97 & 0.96 \\
\hline $36 \_2$ & $2.079 \mathrm{E}-5$ & 1.06 & 1.04 & 1.06 & 1.07 & 1.02 & 1.05 & 1.05 & 1.05 \\
\hline $36 \_3$ & $1.318 \mathrm{E}-5$ & 1.06 & 1.04 & 1.07 & 1.06 & 1.02 & 1.05 & 1.06 & 1.04 \\
\hline $48 \_1$ & $1.954 \mathrm{E}-5$ & 1.00 & 1.03 & 1.06 & 1.03 & 1.01 & 1.06 & 1.03 & 1.03 \\
\hline $48 \_2$ & $7.61 \mathrm{E}-6$ & 1.06 & 1.06 & - & 0.96 & 0.954 & - & 1.06 & 0.96 \\
\hline $48 \_3$ & $4.57 \mathrm{E}-6$ & 1.09 & 1.06 & - & 1.00 & 0.96 & - & 1.06 & 0.98 \\
\hline $48 \_4$ & $2.53 \mathrm{E}-6$ & 1.09 & 1.09 & - & 1.04 & 1.02 & - & 1.09 & 1.03 \\
\hline $60 \_1$ & $2.05 \mathrm{E}-6$ & 1.06 & 1.01 & 1.06 & 1.09 & 1.03 & 1.09 & 1.04 & 1.07 \\
\hline $60 \_3$ & $7 \mathrm{E}-07$ & 1.03 & 0.98 & 1.02 & 1.03 & 0.98 & 1.02 & 1.01 & 1.01 \\
\hline \hline Av.Ag & - & 1.05 & 1.03 & 1.04 & 1.03 & 0.99 & 1.04 & 1.04 & 1.01 \\
\hline$\sigma$ & - & 0.03 & 0.04 & 0.04 & 0.04 & 0.04 & 0.04 & 0.04 & 0.03 \\
\hline
\end{tabular}

TABLE 16: This table shows the extracted DIS Cross-Section from a previous study by A. Stefanko $\left(A_{\text {old }}\right)$ and B. Karki $\left(B_{\text {old }}\right.$ and $\left.E_{\text {old }}\right)$ with a New study I did using the same DIS Cross section normalizations but a standardized yield analysis. All cross sections are expressed as a fraction $\frac{\sigma_{i}}{\sigma_{s}}$ where $\sigma_{i} \in(\mathrm{A}, \mathrm{B}, \mathrm{E})$ and $\sigma_{s}$ is the reference cross-section 


\section{CHAPTER 6}

\section{DVCS CALORIMETER AND PHOTON SELECTION}

DVCS3 experiment utilizes an electro magnetic calorimeter made up of $208 \mathrm{PbF}_{2}$ blocks capacitively coupled to fast response PMT and read by $1 \mathrm{GHz}$ Analogue Ring Sampler (ARS) for photon detection, discussed in section 3.4. ARS records full signal pulses similar to a digital oscilloscope. Initially a waveform analysis algorithm shifts through the recorded ARS data to extract the timing and energy information for each signal pulse registered. The amplitude and time information are then calibrated to optimize energy and timing resolutions. Then this calibrated data is analyzed by a clustering algorithm to find clusters in the calorimeter and the timing and position information for the detected photon is resolved from the centroid block information and energies of each blocks in a cluster are summed to find the detected photon energy.

Following calibration steps are necessary before the calorimeter can be used to construct the final photon information using the clustering analysis.

- Energy Calibration

- Cosmic Calibration : Pre-Beam calibration to adjust the phototube gains by adjusting the High Voltage

- Elastic Calibration : Absolute calibration using elastic data

$-\pi^{0}$ calibration : Monitoring the energy calibration throughout the run period

- HRS-Calorimeter coincidence time correction : To better match the photon detected in the Calorimeter with the electron in the HRS.

\subsection{WAVEFORM ANALYSIS}

Shape of the PMT response signals can be assumed to be characteristics of the PMT and independent of signal amplitude. A waveform analysis algorithm can then analyze the ARS signal to find these signal pulses and fit it with a reference shape to extract the arrival time and signal amplitude of the pulse. Reference shapes for the pulses are determined for each 
individual calorimeter block from elastic $e p \rightarrow e^{\prime} p^{\prime}$ data where the signals are clean due to the low probability of pileup. The algorithm initially performs a baseline fit to identify any signal to fit using a flat baseline $b(t)$ for any given arrival time $t$. When a signal is found then the signal $\left\{x_{i}\right\}$ is fit with a one pulse reference shape $\left\{h_{i}\right\}$ to extract the amplitude $a(t)$ by minimizing,

$$
\chi^{2}(t)=\sum_{i_{\min }}^{i_{\max }}\left(x_{i}-a(t) h_{i-t}-b(t)\right)^{2}
$$

Here, the amplitude of the pulse $a(t)$ and it's arrival time $t$ are free parameters. \{ $\left.i_{\min }, i_{\max }\right\}$ is the analysis time window, which is smaller than the full $128 \mathrm{~ns}$ window of the ARS to minimize the effects from accidental pulses. When the minimum $\chi^{2}(t)$ evaluated is above a set analysis threshold, then a two pulse reference shape is fitted and the arrival time and amplitude of the two pulse is obtained by minimizing the following $\chi^{2}\left(t_{1}, t_{2}\right)$ for each combination of $t_{1}$ and $t_{2}$.

$$
\chi^{2}\left(t_{1}, t_{2}\right)=\sum_{i_{\min }}^{i_{\max }}\left(x_{i}-a_{1}\left(t_{1}, t_{2}\right) h_{i-t_{1}}-a_{2}\left(t_{1}, t_{2}\right) h_{i-t_{2}}-b\left(t_{1}, t_{2}\right)\right)^{2}
$$

Waveform analysis algorithm is initially developed by C. Muñoz Camacho [18] for the first Hall-A DVCS experiment in 2004. Mongi Dlamini [28] and Frédéric Georges[33] performed the analysis for the DVCS3 data independently using improved versions of the same algorithm. Figure 44 shows an example of a one and a two pulse fit samples.

\subsection{CALORIMETER ENERGY CALIBRATION}

The goal of the energy calibration is to adjust the gain of each individual phototube so that the signal response of the individual PMTs matches each other across all 208 blocks. This goal is achieved in three distinct calibration steps.

\subsubsection{COSMIC RAY CALIBRATION}

Cosmic rays were used to calibrate and adjust the high voltage (HV) on each PMTs to give a first order gain matching before the actual beam run. Cosmic runs were taken by triggering the data acquisition using the coincidence between two large plastic scintillators placed on the top and the bottom of the calorimeter indicating a cosmic ray passing through the calorimeter. Cosmic ray is a minimum ionizing particle and deposit about $35 \mathrm{MeV}$ per calorimeter block when traveling vertically through the $3 \mathrm{~cm}$ distance as the energy 


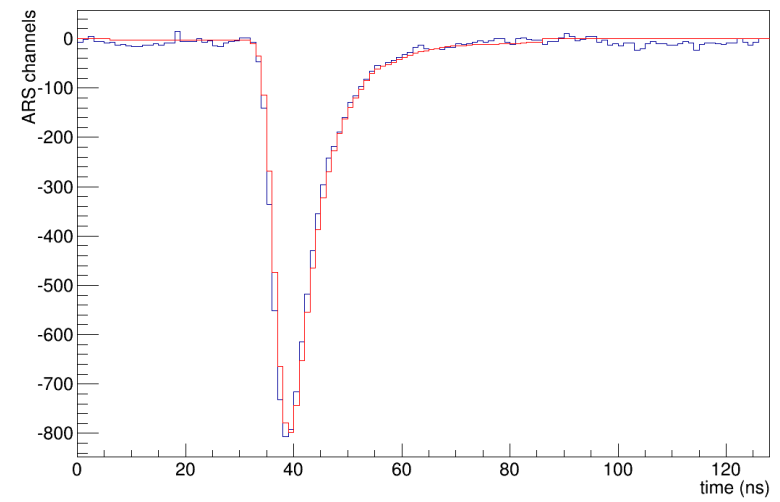

(a) Single pulse fit

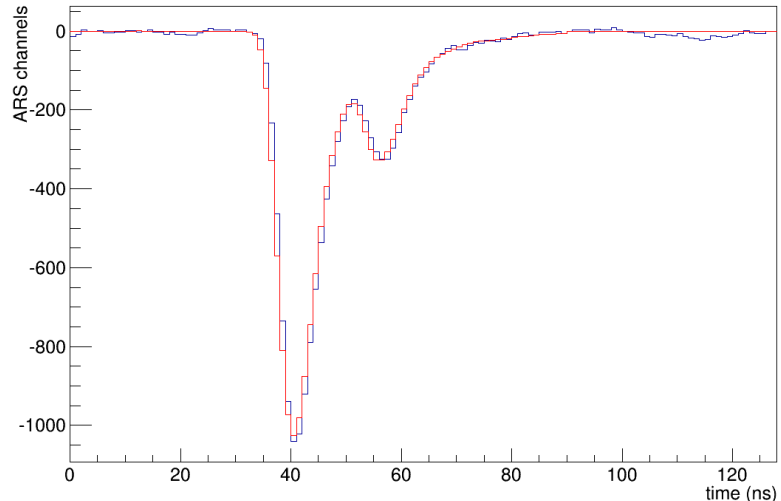

(b) Two pulse fit

FIG. 44: Figure shows the one pulse and two pulse fit sample events. The signal pulse is is in blue and the fit using the reference shape is in red. This results are courtesy of Frédéric Georges from 33 ]

deposition is a function only of the distance travelled. It will produce about 35 Cherenkov electron. To make sure that the cosmic ray travelled vertically, and hence same distance through each block, a vertical cosmic cut which requires each selected cosmic event to have deposited energy in both top and the bottom block of the same column can be employed. The amplitude spectra of each block can then fitted using a gaussian function and the high voltage can be adjusted so that the signal mean is centered around a common value for all PMTs using equation 79 in several iterations.

$$
\begin{gathered}
G=\alpha H V^{\beta} \\
H V_{2}=H V_{1}\left(\frac{G_{1}}{G_{2}}\right)^{\frac{1}{\beta}}
\end{gathered}
$$

The method yielded a 4.5\% cross-calibration for ARS signal and 5.3\% for the ADC signals [28].

\subsubsection{ELASTIC CALIBRATION}

The absolute energy calibrations are performed using the elastic $e p \rightarrow e^{\prime} p^{\prime}$ data. In this step the polarity of the High Resolution Spectrometer was reversed so that the recoiling proton is detected in the spectrometer and the scattered electron is detected in the DVCS 
calorimeter. In order to circumvent the limitation posed by the small acceptance of the spectrometer in fully illuminating the calorimeter, the calorimeter was placed 6 meters from the target in three different angles. The kinematics of the elastic scattering is known so that the expected energy of the electron can be computed from the energy of the detected proton using equation 80. We can define the reconstructed electron energy in the calorimeter as in equation 81 then optimizing the $\chi^{2}$ given in equation 82 will provide the optimized block co-efficient $C_{j}$ to reconstruct the energy deposited in each block.

$$
\begin{array}{lcc}
E_{i}^{e}= & E_{B}+M_{P}-E_{i}^{P} \\
E_{i}^{e}= & \sum_{j=0}^{208}\left(C_{j} A_{j}^{i}\right) \\
\chi^{2}= & \sum_{i=0}^{N}\left(E_{i}^{e}-\sum_{j=0}^{208}\left(C_{j} A_{j}^{i}\right)\right)^{2}
\end{array}
$$

$E_{i}^{e}$ is the scattered electron energy of the $i^{t h}$ event. $E_{B}$ is the beam energy, $M_{P}$ is the proton mass and $E_{i}^{P}$ is the detected recoil proton energy of the $i^{\text {th }}$ event. $C_{j}$ is the block co-efficient and $A_{j}^{i}$ is the signal amplitude of the block $\mathrm{j}$ for the event $\mathrm{i}$.

The calibration is performed at the beginning and closer to the end of each run period. An energy resolution of $2.39 \%$ was measured at $4.2 \mathrm{GeV}$ during Fall 2016 and $4.94 \%$ was measured at 5.0 GeV during Fall 2014 [28].

\subsection{3 $\pi^{0}$ CALIBRATION}

The close proximity of the calorimeter to the beamline resulted in rapid radiation damage to the calorimeter blocks resulting in gain losses requiring frequent adjustment to the blockcoefficients in order to keep up with the gain losses. As discussed in the previous section, elastic calibration requires the reversal of the polarity in the HRS and hence an invasive process during which production data cannot be taken. Moreover the process is lengthy, consuming up to one whole day of beam time. Hence the process is done only in affordable intervals. That required a complimentary calibration procedure to monitor and optimize for the gain losses.

R.T. Jones et al., [49] developed a calibration method for Pb-Glass calorimeter that doesn't require a beam of known energy by detecting all decay photons from a meson and optimizing the function given in equation 83 .

$$
\mathcal{F}=\sum_{i=1}^{N}\left(m_{i}^{2}-m_{0}^{2}\right)^{2}+2 \lambda \sum_{i=1}^{N}\left(m_{i}^{2}-m_{0}^{2}\right)
$$

Here, the index $i$ denotes the $i^{\text {th }}$ calibration event, $m_{i}$ and $m_{0}$ are the reconstructed and the 
rest mass of the meson respectively and $\lambda$ is a Lagrange multiplier to enforce the constrain of $\left\langle m_{i}\right\rangle=m_{0}$

The detected $\pi^{0}$ photon pair from the $e p \rightarrow e^{\prime} p^{\prime} \pi^{0} \rightarrow e^{\prime} p^{\prime} \gamma \gamma$ reaction can be used to establish a calibration procedure internally called $\pi^{0}$ calibration by reconstructing the $\pi^{0}$ invariant mass. After finding the photon clusters, for an event with only two clusters in the calorimeter, energies of all blocks in the the individual clusters can be summed to find the individual photon momentum values and the cluster position information can give the opening angle between them. $\pi^{0}$ invariant mass then can be reconstructed using equation 84.

$$
m_{i}^{2}=2 p_{\gamma_{1}} P_{\gamma_{2}}\left(1-\cos \left(\theta_{\gamma_{1} \gamma_{2}}\right)\right)
$$

A block dependent gain correction factor $\epsilon_{k}$ can be introduced in to the photon momentum reconstruction as follows

$$
p_{i j}=\sum_{k=0}^{208}\left(1+\epsilon_{k}\right) E_{i j}^{k}
$$

Here, $j \in\left\{\gamma_{1}, \gamma_{2}\right\}, i$ is the event index, $k$ is the block index and $E_{i j}^{k}$ is the reconstructed energy of the given block in the cluster $\mathrm{j}$ for the $i^{t} h$ event. Minimizing the function given in equation 83 with respect to $\epsilon_{k}$ will yield

$$
\epsilon_{k}=\left[C_{k k^{\prime}}^{-1}\right](D-\lambda L)_{k}^{\prime}
$$

with,

$$
\begin{array}{rcc}
C_{k k^{\prime}} & = & \sum_{i=1}^{N}\left(\frac{\partial m_{i}^{2}}{\partial \epsilon_{k}} \frac{\partial m_{i}^{2}}{\partial \epsilon_{k}^{\prime}}\right) \\
D_{k}= & -\sum_{i=1}^{N}\left(\left(m_{i}^{2}-m_{0}^{2}\right) \frac{\partial m_{i}^{2}}{\partial \epsilon_{k}}\right) \\
L_{k}= & \sum_{i=1}^{N} \frac{\partial m_{i}^{2}}{\partial \epsilon_{k}}
\end{array}
$$

Figure 45 from [33] shows the effectiveness of $\pi^{0}$ calibration method in compensating for the gain loss during the run period.

\subsection{HRS-CALORIMETER COINCIDENCE TIME CORRECTION}

In our analysis we not only need to detect a photon, but also need to make sure that the detected photon is in fact from the same event as the detected electron. Since we detect the electron in the spectrometer and the photon in the calorimeter, it is very important to measure the coincidence timing between the two with good timing resolution. This will not only play a role in minimizing the accidentals but also will improve the reconstructed 


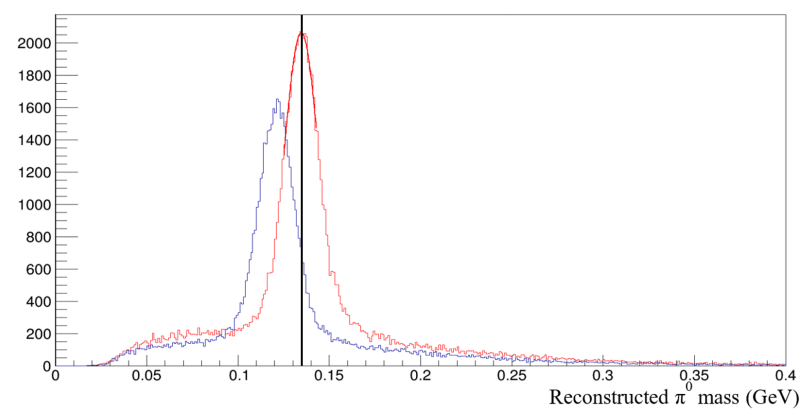

FIG. 45: Figure shows the reconstructed $\pi^{0}$ invariant mass before (blue) and after (red) the $\pi^{0}$ calibration for a fall 2016 data performed by Frédéric Georges [33]. The black reference line indicates the expected $\pi^{0}$ mass. The result demonstrate that the gain loss due to radiative effects are successfully corrected using the $\pi^{0}$ calibration.

missing mass resolution. The arrival time of a signal varies between the ARS channels (calorimeter blocks) as well as event by event mainly due to the cabling effect. Calibrating the arrival times of individual ARS channels to a common value would enable us to use a global coincidence time window rather than a block dependent time window.

Following factors are calibrated in order to achieve a good coincidence timing

- Trigger Jitter : Jitter in the ARS Stop signal

- S2 timing correction: Timing correction for the 16 scintillator paddles of the S2 detector compensating for any cable issues as well as the photon travel time correction to compensate the timing offset due to the hit position.

- Electron travel time correction : Travel time variation through the spectrometer due to the dispersive angle and momentum variation.

Figure 46 fro [28] shows the results of the coincidence time correction performed by Mongi Dlamini for DVCS3 experiment.

\subsection{CLUSTERING ALGORITHM}

$\mathrm{PbF}_{2}$ blocks used in the DVCS Calorimeter has very small Moliére radius. This implies $90 \%$ of the energy from a single photon is deposited in the central block. Still a considerable amount of energy is shared with neighboring blocks. The goal of the clustering algorithm is to construct photon energies from the block informations saved during the waveform analysis. When a cluster is detected, the cluster arrival time is computed as an energy weighted sum of 


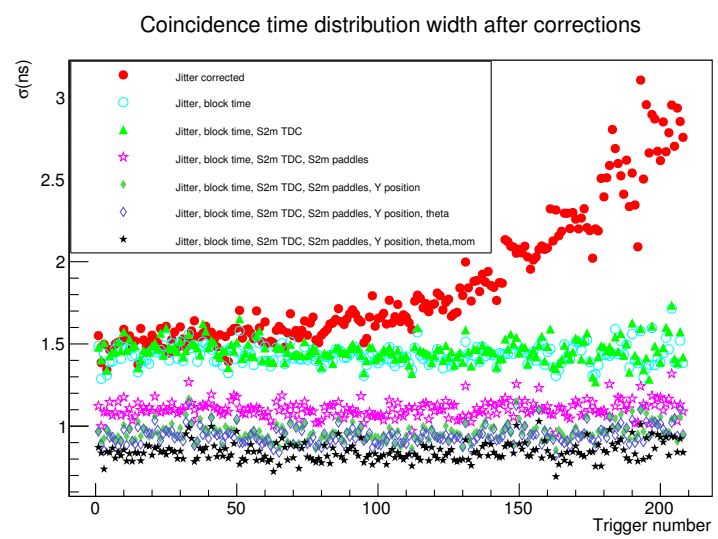

FIG. 46: Figure shows the width of the timing distribution per calorimeter block after each consecutive timing correction. The black star spectrum indicates the final corrected time distribution. Timing correction for DVCS3 was performed by Mongi Dlamini[28]

block signal arrival times, the photon energy is computed as the sum of the energies of each block in the cluster and the impact position is computed as the logarithmically weighted sum of block positions as follows,

$$
\begin{array}{rc}
E_{\gamma}= & \sum_{i \in \text { cluster }} C_{i} A_{i} \\
t_{\gamma}= & \frac{\sum_{i} A_{i}\left(t_{i}-t_{\text {corr }}\right)}{\sum_{i} A_{i}} \\
R_{\gamma}= & \frac{\sum_{i} w_{i} R_{i}}{\sum_{i} w_{i}} ; R_{\gamma} \in\left\{x_{\gamma}, y_{\gamma}\right\} \\
w_{i}= & \operatorname{Max}\left\{0,\left[W_{0}+\ln \left(\frac{C_{i} A_{i}}{E_{p}}\right)\right]\right\}
\end{array}
$$

Here $C_{i}$ are the energy calibration co-efficient and $A_{i}$ is the signal amplitude of the block. When blocks are considered for a cluster, the base noise present in the blocks could contribute to an over estimation of the photon energy, to remedy this, the neighboring blocks are analyzed in a $2 \times 2$ combinations and only kept for the cluster if the combination passed a calorimeter cluster threshold. Waveform analysis preserves up to 2 pulses per event per calorimeter block. When this happens, the pulse with the arrival time closes to zero is kept in the cluster. The clustering algorithm uses an infection algorithm similar to the one described in section 4.4.5. In this analysis I apply lower clustering threshold and keep up to 9 clusters (photons) per event

When additional photons arrive at the calorimeter, they can be separated from the previous photons in space or in time. These photons could be coming from a real process like a $\pi^{0}$ decay or accidental coincidences. The clustering algorithm has to distinguish between 
the many separate clusters formed by these separate photons. CEBAF beam has a pulse frequency of $249.5 \mathrm{MHz}$ during 4 hall operations giving rise to a 4 ns time structure in the beam. In order to minimize the complication in clustering due to the random coincidence events coming from a nearby beam bunch, the clustering is done within a small coincidence time window. The central coincidence time window is determined to be $[-3 n s, 3 n s]$.

Since the calorimeter is placed only a meter away from the target, the position coordinates obtained in equation 92 need to be corrected for the vertex position as well as the shower depth.

$$
\delta R_{\gamma}=R_{\gamma}\left(1-\frac{a}{\sqrt{L_{v c}^{2}+R_{\gamma}^{2}}}\right) ; R_{\gamma} \in\left\{x_{\gamma}, y_{\gamma}\right\}
$$

$W_{0}$ and $a$ can be optimized by using a Monte-Carlo simulation and then using elastic data. An optimized value of $a=7 \mathrm{~cm}$ and $W_{0}=4.3$ was used in the first DVCS experiment [18]. A more accurate representation of the shower depth accounted for the energy dependency is used in the DVCS3 experiment given in equation 95

$$
a=0.30 E_{\gamma}^{0.28}+4.862
$$

\subsection{PHOTON SELECTION}

As discussed in the previous section, the clustering analysis is done in separate time windows. Figure 47 shows the full timing spectrum (blue) and the different clustering time windows (red). Only the central bucket is analyzed for DVCS photon candidates. The left and right buckets are used to estimate the accidental coincidence background in the true coincidence window.

\subsubsection{CALORIMETER FIDUCIAL CUT}

When the photon hits the edges of the calorimeter, some leakage of energy could happen, this could result in an under estimate of the detected photon energy. To avoid this situation a calorimeter boundary for good photon reconstruction is defined as follows.

$$
\begin{gathered}
-20 \leq x \leq x_{\max } \\
-20 \leq y \leq 20 \\
-33 \leq x+y \leq 24 \\
-24 \leq y-x \leq 33
\end{gathered}
$$




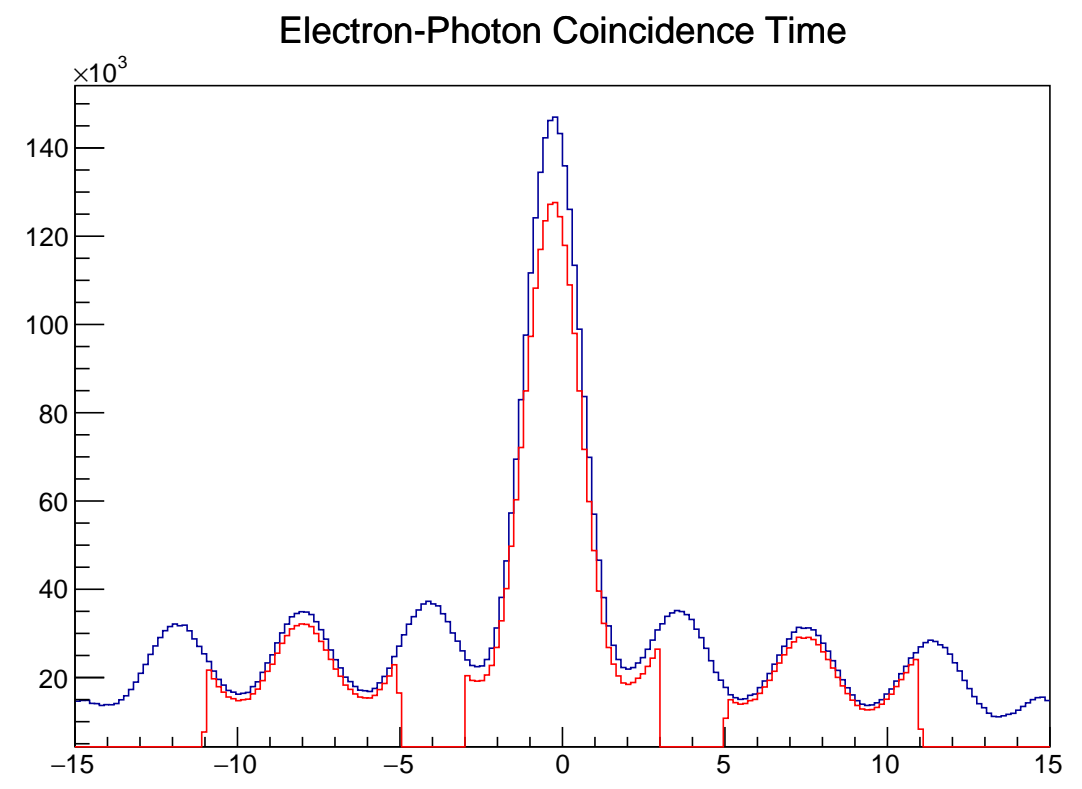

FIG. 47: Figure shows the coincidence time spectrum of all detected photons. The blue spectrum is obtained by clustering in the whole $[-15,15] \mathrm{ns}$ window. The red spectrum is obtained by doing the clustering in each individual timing windows of $[-3,3] \mathrm{ns},[-11,5] \mathrm{ns}$ and $[5,11] \mathrm{ns}$. Red spectrum includes an additional selection in the calorimeter boundaries.

Here $x_{\max }=7.5$ for Kin 484 and $x_{\max }=11$ for all other kinematics.

\subsubsection{PHOTON ENERGY CUT}

On top of the clustering threshold a kinematic dependent cut on the photon minimum energy is applied to eliminate low energy photons. Table 17 summarizes the minimum photon energy cut values.

\subsection{CONTAMINATION SUBTRACTION}

The DVCS photon candidates selected after applying above mentioned cuts still would have contaminations coming from the accidental coincidences and the $\pi^{0}$ decays. These accidentals should be estimated and subtracted to obtain a clean missing mass spectrum.

\subsubsection{ACCIDENTAL SUBTRACTION}

As briefly mentioned in section 6.4 a random photon from a different event or process can make an accidental coincidence with the electron detected in the HRS. This background will 


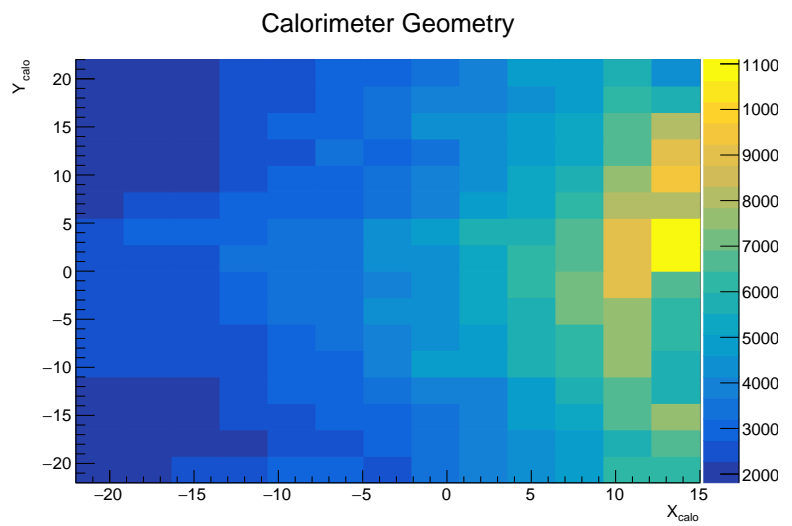

(a) Full calorimeter

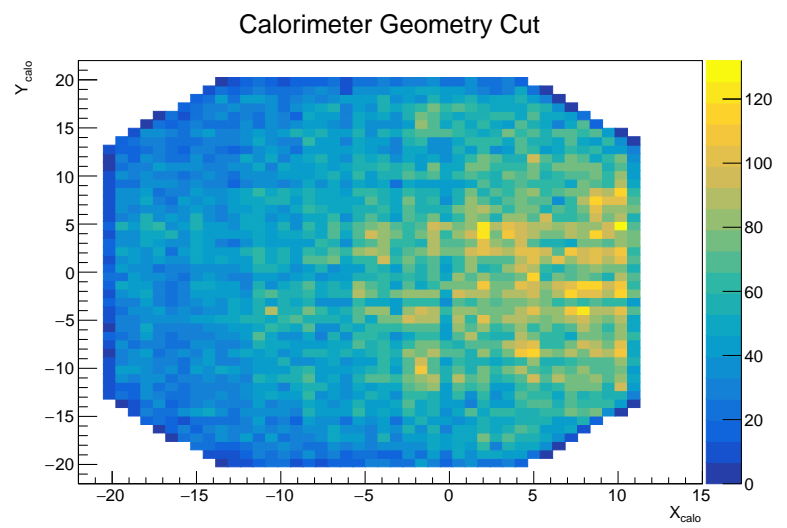

(b) Accepted region for photon selection

FIG. 48: Figure (a) shows the full calorimeter and (b) shows the calorimeter fiducial region for photon selection, for Kin 483. (b) is more finely binned than F(a).

\begin{tabular}{|c|c|c|c|c|c|}
\hline Kinematic & Run Range & $E_{\gamma}^{\text {min }} \mathrm{GeV}$ & Clust.Threshold GeV & $M_{\pi^{0}}$ & $\sigma_{M_{\pi^{0}}}$ \\
\hline $36 \_1$ & {$[10370,10649]$} & 3.0 & 1.1 & 0.134286 & 0.0102703 \\
\hline $36 \_2$ & {$[14147,14261]$} & 4.24 & 1.6 & 0.135402 & 0.00972696 \\
\hline $36 \_3$ & {$[4471,14525]$} & 4.8 & 1.6 & 0.134976 & 0.00947850 \\
\hline $48 \_1$ & {$[2508,12661]$} & 2.0 & 0.5 & 0.135588 & 0.0115915 \\
\hline $48 \_2$ & {$[13000,13015]$} & 3.0 & 0.9 & 0.134976 & 0.0108733 \\
\hline $48 \_3$ & {$[12838,12999]$} & 4.0 & 1.1 & 0.134379 & 0.0100754 \\
\hline $48 \_4$ & {$[113100,13162]$} & 5.8 & 1.1 & 0.135058 & 0.0110005 \\
\hline $60 \_1$ & {$[14267,14470]$} & 2.8 & 0.8 & 0.134418 & 0.0115311 \\
\hline $60 \_3$ & {$[14526,14950]$} & 5.0 & 1.0 & 0.134219 & 0.0109747 \\
\hline $48 \_2$ & {$[13183,1326]$} & 3.0 & 0.9 & 0.134976 & 0.0108733 \\
\hline $48 \_4$ & {$[13279,13418]$} & 5.8 & 1.5 & 0.135560 & 0.00984475 \\
\hline $60 \_1$ & {$[14951,15177]$} & 2.8 & 0.8 & 0.134418 & 0.0115311 \\
\hline
\end{tabular}

TABLE 17: This table summarizes the photon energy cut, Calorimeter cluster threshold, $\pi^{0}$ invariant mass $M_{\pi^{0}}$ and $\sigma_{M_{\pi^{0}}}$ for each run period of each kinematics. 
be distributed uniformly across the different time windows. Selecting a time window with the same width as the true coincident time window well outside of the it will enable us to estimate this background. The beam has a time structure of $4 \mathrm{~ns}$ as mentioned in section 6.4 and can be seen in Figure 47. We select the accidental time window such that it is at least $8 \mathrm{~ns}$ apart from the tru coincidence time window in order to account for this time structure of the beam.

We select two such time windows $[-11,5]$ ns and $[5,11]$ ns. We then apply all photon selection cut as similar to the DVCS photon and estimate the events in each of this time intervals and then average it over. These events are then subtracted from the DVCS canditates. Figure 47 shows the coincidence time spectrum and the different time windows chosen to estimate the accidentals.

\subsection{2 $\pi^{0}$ SUBTRACTION}

A $\pi^{0}$ produced discussed in section 2.4.1, will decay into two photons with a branching ratio of 0.99 as $\pi^{0} \rightarrow \gamma \gamma$. in the rest frame of the pion these two photons will have $\frac{1}{2}$ of the $\pi^{0}$ invariant mass and travel in opposite directions to conserve momentum. In the laboratory frame however, the decay photons can travel asymmetrically and one of the two photons can escape detection either due to the finite angular acceptance of the calorimeter or in some extreme cases, the forward traveling photon can contain almost all of the $\pi^{0}$ invariant mass and appear like a DVCS photon and the other other photon can escape detection. A Monte-Carlo based statistical subtraction method is developed for handling these situations.

I first detect a $\pi^{0}$ candidate from up-to 9 photon candidates. An algorithm systematically goes through all 9 photons and look for photons that are inside a slightly larger fiducial region in the calorimeter defined in equation 97 and match them up in pairs until it detects a photon pair that is compatible with $\pi^{0}$ invariant mass for the kinematics within $3 \sigma$ listed in Table 17.

$$
\begin{aligned}
& -21 \leq x \leq 12 \\
& -21 \leq y \leq 21
\end{aligned}
$$

For each of the detected $\pi^{0}$, the algorithm then randomly generates $5000 \pi^{0}$ events and computes the decay pairs in $\pi^{0}$ rest frame and then boost it in the laboratory frame corresponding to the detected $\pi^{0}$ momentum. The photons are then propagated to the calorimeter and the instances of $0\left(n_{0}\right), 1\left(n_{1}\right)$ or both $\left(n_{2}\right)$ photons being detected in the calorimeter is tracked. 
For the cases of only one of the photon is detected, all experimental variables pertaining to the detected photons are computed and written out to a root file as if they are actual photons detected experimentally. Then these photons are analyzed identically to the DVCS data and subtracted from the data with a weighting factor of $W=\frac{1}{5000} \times \frac{n_{0}+n_{1}+n_{2}}{n_{2}}=\frac{1}{n_{2}}$.

Figure 49a shows a typical reconstructed missing mass spectrum (Black), the estimated contamination (accidental in Magenta and $\pi^{0}$ in Green) and The contamination subtracted spectrum (Blue). Subtracted spectrum shows a gaussian distribution centered around the expected value of $M_{P}^{2}$.

\subsection{MONTE-CARLO EVENT GENERATION AND GEANT4 SIMULATION}

We use a geant4 simulation to estimate the phase-space of each event. A MonteCarlo event generator generates random DVCS events in the five fold kinematic space $\left\{Q^{2}, x_{B},\left(t-t_{\text {min }}\right), \phi_{e}, \phi_{\gamma \gamma}\right\}$. The reaction vertex is randomly generated. The electron and photon kinematics at the vertex is then computed after applying the external radiative correction mentioned in section 5.5.1. The particles are then propagated through the major structures of the DVCS experiment including the target holder, reaction chamber, various shieldings, HRS entrance and DVCS calorimeter using a geant4 simulation. The electron's acceptance in the HRS is determined by the R-Functions mentioned in section 4.5. DVCS Calorimeter is fully implemented, after running the generated photon through the calorimeter, the clustering algorithm mentioned in section 6.4 will analyze the information from the calorimeter blocks and reconstruct the photon clusters as if they are real DVCS events.

The phase-space of each event $\Gamma_{i}^{D V C S}$ is computed event by event using equation 98 and recorded in the data stream with all other relevant kinematics.

$$
\Gamma_{i}^{D V C S}=\Delta Q^{2} \Delta x_{B} \Delta\left(t-t_{\text {min }}\right) \Delta \phi_{\gamma \gamma} \Delta \phi_{e}
$$

Vertex kinematics generated here are used to compute the Kinematic dependencies in the BMK terms mentioned in equation 120.

\subsection{CALIBRATION AND SMEARING OF THE SIMULATION}

The geant4 simulation mentioned above does not include the simulation for the energy and angular resolution. In a real experiment there are always more variables contributing to the resolution effect than a simulation can reasonably account for. Moreover $4 \%$ of photon energy leaks between and behind the calorimeter crystals. While the calibration process 
discussed in section 6.2 compensate for this loss in real data, the simulation doesn't do that. This results in the missing mass peak is centered at a higher than expected position. We use a post smearing algorithm to smear the simulation by a gaussian distributed random deviate.

$\overrightarrow{q^{\prime}} \rightarrow \operatorname{Gauss}(\mu, \sigma) \times \overrightarrow{q^{\prime}}$. Here $\overrightarrow{q^{\prime}}$ is the four-momentum of the generated real photon $\overrightarrow{q^{\prime}}=\left(E_{q}, q_{x}, q_{y}, q_{z}\right)$ and $\mu$ is the calibration coefficient and $\sigma$ is the smearing coefficient.

The calorimeter crystals suffered some fast darkening which affected different crystals differently. To handle this, the calorimeter is divided in to a $4 \times 4$ grid and a locally optimized $\mu$ and $\sigma$ are found for each calorimeter zone for each kinematic settings individually by fitting the missing mass distribution of the simulation to the real data. Figure 49 shows the smeared missing mass spectrum for Kin 481.

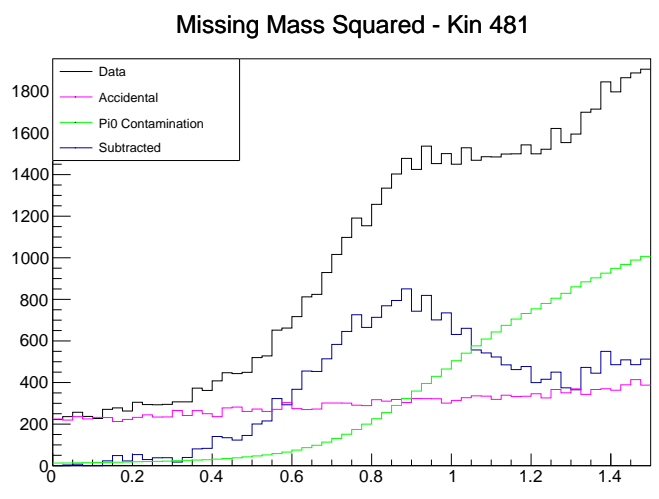

(a) Missing Mass Subtraction

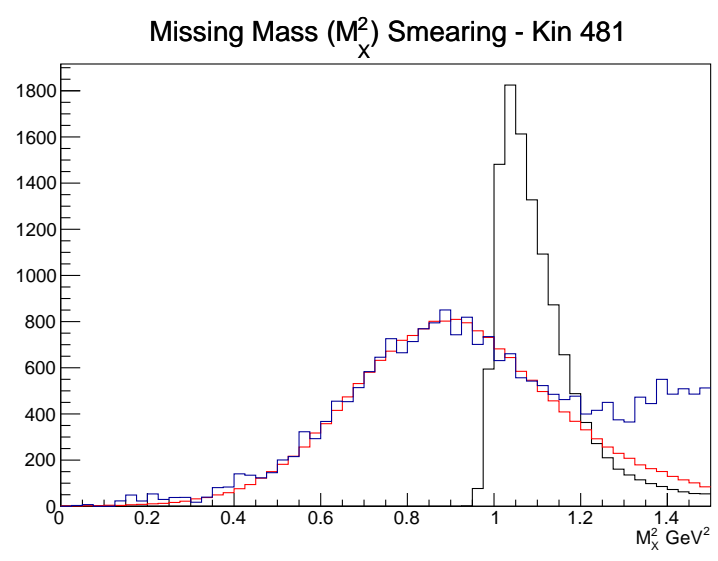

(b) Simulation smearing

FIG. 49: Left figure shows the reconstructed missing mass spectrum for kin 481 in Black. This spectrum includes the contamination from accidental coincidences (Magenta) and when only one photon is detected from a $\pi^{0}$ decay pair (Green spectrum is simulated estimate of this case). The blue spectrum is from the estimated DVCS event candidates after the contamination subtraction (Black - Green - Magenta). Right figure shows missing mass spectrum from the simulation after smearing (Red) overlaid on top of the missing mass subtracted spectrum of the data (Blue). The black spectrum in the left plot is the simulated missing mass before smearing.

\subsection{CALORIMETER MULTI CLUSTER, MISSING MASS AND EXCLUSIVITY}

The next important step in selecting exclusive DVCS events is to identify the recoil proton. In DVCS3 experiment we do not detect the final state proton, rather we employ a missing mass cut to identify the proton and ensure the exclusivity of the DVCS event. 
In the reaction $e p \rightarrow e \gamma X$, where $X$ is the undetected final state, the missing mass $M_{x}$ can be given by the formula $M_{x}^{2}=\left(\vec{k}+\vec{P}-\overrightarrow{k^{\prime}}-\overrightarrow{q^{\prime}}\right)^{2}$, where $\vec{k}, \overrightarrow{k^{\prime}}, \vec{P}, \overrightarrow{q^{\prime}}$ are the four-momenta of the initial electron, initial proton, scattered electron and the final state photon. For the fixed target experiment of ours, the initial four-vectors would be $\vec{P}=\left(M_{P}, 0,0,0\right)$ and $\vec{k}=$ $\left(E_{\text {beam }}, 0,0, E_{\text {beam }}\right)$ after neglecting the electron mass. The final state four-vectors $\overrightarrow{k^{\prime}}$ and $\overrightarrow{q^{\prime}}$ are constructed from the detected electron and the photon, where $M_{P}^{2}=0.88 G e V^{2}$. Figure 49 shows the reconsrtucted missing mass spectrum for kin 481.

\subsubsection{MISSING MASS CUT}

In order for the detected process to be exclusive DVCS, the reconstructed missing mass should be that of a proton. In reality due to the resolution effects and energy reconstruction uncertainties, the reconstructed missing mass spectrum would be a gaussian centered around the expected value, rather than a delta function.

Missing Mass Squared - Kin 481

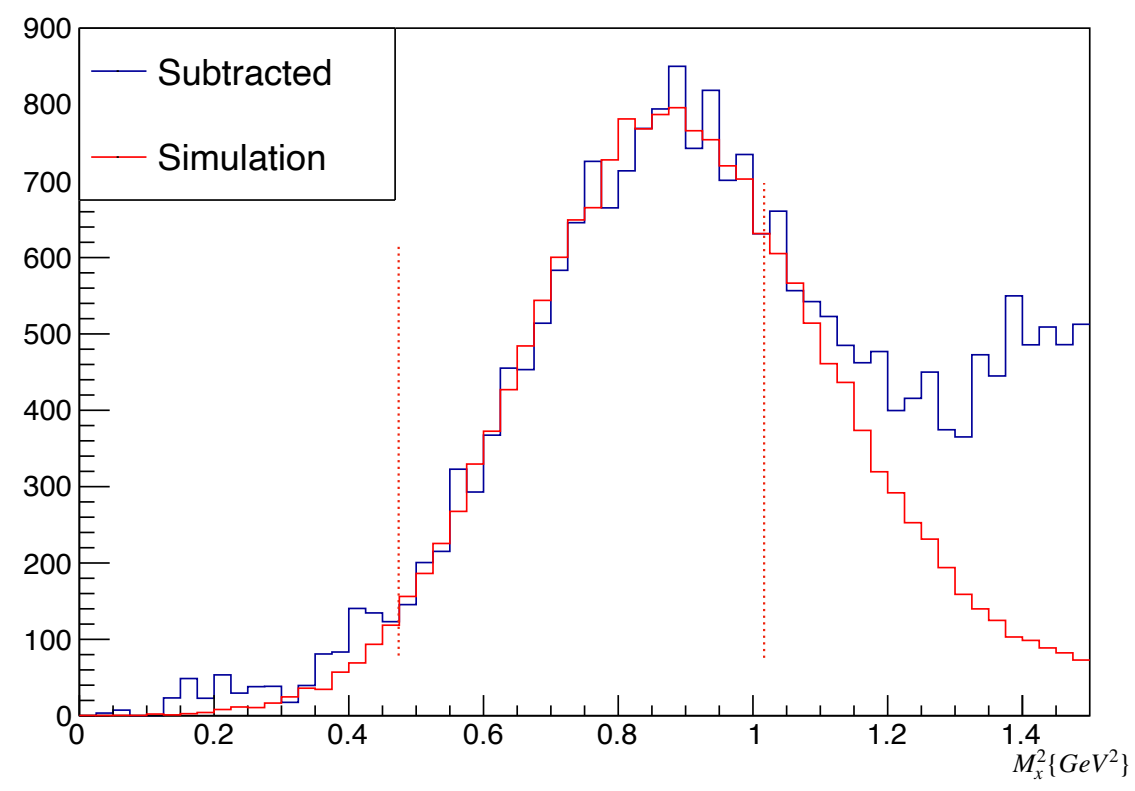

FIG. 50: Plot shows missing mass spectrum from the simulation after smearing (Red) overlaid on top of the missing mass subtracted spectrum of the data (Blue). Red dotted lines are indicating the missing mass range for photon selection.

Figure 50 shows a typical contamination subtracted missing mass spectrum from DVCS data overlaid on the smeared simulated missing mass spectrum. The contamination subtracted spectrum shows a gaussian distribution centered around the expected value of $M_{P}^{2}$. We take the region in which both spectra show acceptable match as the missing mass cut 


\begin{tabular}{|c|c|c|}
\hline Kin & $M x_{\text {cut }_{\min }}^{2}$ & $M x_{\text {cut }_{\max }}^{2}$ \\
\hline $36 \_1$ & 0.35 & 1.10 \\
\hline $36 \_2$ & 0.35 & 1.10 \\
\hline $36 \_3$ & 0.45 & 1.075 \\
\hline $48 \_1$ & 0.45 & 1.10 \\
\hline $48 \_2$ & 0.45 & 1.10 \\
\hline $48 \_3$ & 0.275 & 1.10 \\
\hline $48 \_4$ & 0.40 & 1.1 \\
\hline $60 \_1$ & 0.575 & 1.05 \\
\hline $60 \_3$ & 0.5 & 1.075 \\
\hline
\end{tabular}

TABLE 18: This table shows the missing mass cut region for each kinematic setting.

region. The Red dotted lines in the figure 50 indicate the chosen cut region for kin 481.

\subsubsection{MISSING MASS SYSTEMATIC UNCERTAINTY}

The associated DVCS discussed in section 2.4.1 is only separated from the exclusive DVCS by the very small $\pi^{0}$ mass seen in Table 17 . Due to the resolution effects, this process will leak in to the exclusive spectrum even after keeping the maximum of the $M x^{2}$ cut bellow 1.1 GeV. This background is the main systematic uncertainty in this analysis.

Figure 51 shows a study of this systematic uncertainty. We varied one end of the missing mass cut while keeping the other end fixed and extracted the DVCS cross section. The extracted cross section is insensitive to the variation of the low missing mass cut. The high missing mass cut howver clearly shows the background from Associated DVCS. We take the maximum cross section variation within + and $-0.06 \mathrm{GeV}$ region from the central missing mass high cut shown in Table 18 as the systematic uncertainty.

\subsubsection{CALORIMETER MULTI CLUSTER}

DVCS event has only one photon in the final state. Traditional DVCS analysis kept only events with a single calorimeter cluster for the yield analysis and applied a multi-cluster correction estimated with two and three cluster events. Clustering algorithm discussed in section 6.4 also applies a clustering threshold. Lower the threshold is, more multi cluster events we get, and the single cluster requirement will skip over more event as they now got additional lower energy clusters. Higher the threshold we will have less likelihood to get a 

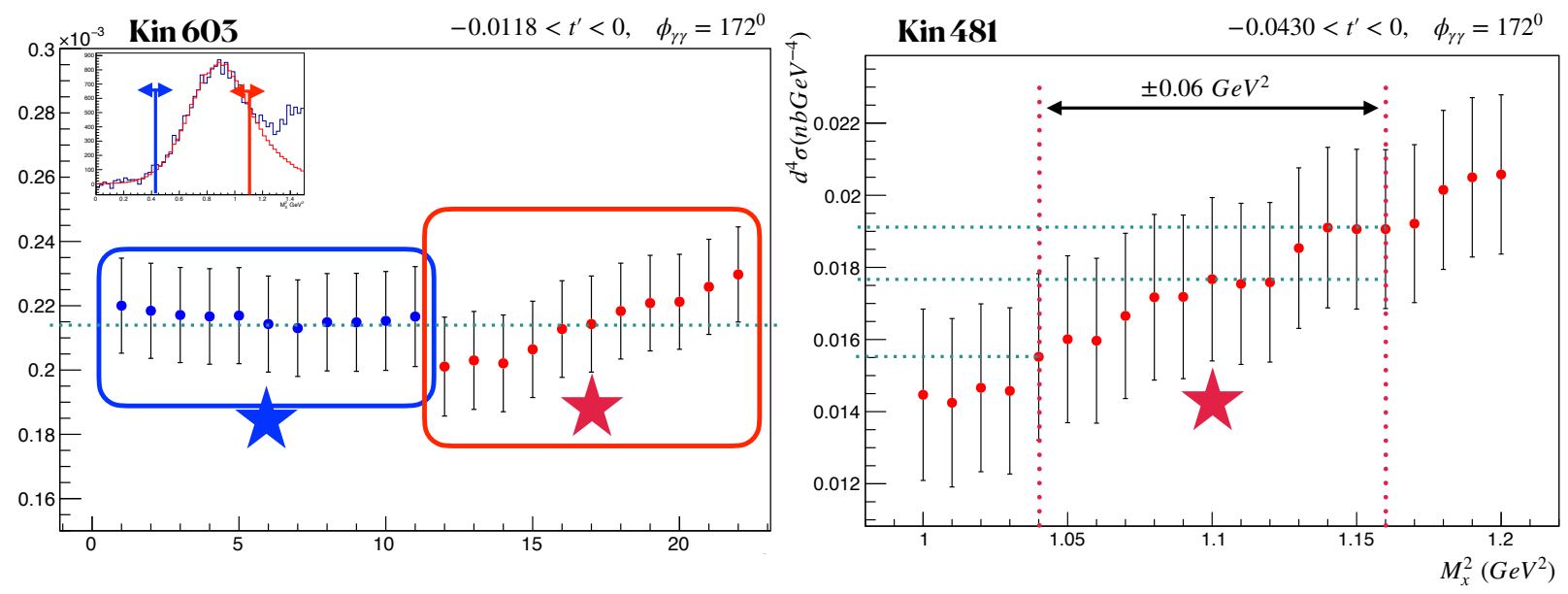

FIG. 51: Left plot shows the extracted cross section at $-0.0118<t^{\prime}<0$, and $\phi_{\gamma \gamma}=172^{0}$ with varied missing mass cut for kin 603 . The blue box extraction are obtained by varying the $M x_{c u t_{\min }}^{2}$ values in $0.2 \mathrm{GeV}$ steps around the central value for the fixed $M x_{c u t_{\text {max }}}^{2}$. The red box extractions are the other way around. Start marked points are the central points. Extracted cross sections are independent of $M x_{c u t_{m i n}}^{2}$ while a strong systematic uncertainty is seen for $M x_{c u t_{\max }}^{2}$. Right plot shows only the red box extraction of left plot with $M x_{c u t_{\max }}^{2}$ varied in $0.1 \mathrm{GeV}$ steps at $-0.0430<t^{\prime}<0$, and $\phi_{\gamma \gamma}=172^{0}$ for kin 481 . The maximum variation between $\pm 0.06 \mathrm{GeV}$ region around the central cut value is taken to be the systematic uncertainty. 
low energy cluster. Thus the yield and the multi cluster correction both becomes functions of the clustering threshold. The situation is complicated even further as we have to lower the clustering threshold and analyze the two cluster events to find the $\pi^{0}$ events to estimate the contamination discussed in section 6.6.2, making the subtraction a function of the clustering threshold as well.

To remedy this my clustering algorithm applies only the lower clustering threshold mentioned in Table 17 and keeps up to 9 clusters. $\pi^{0}$ contamination estimation algorithm uses all 9 clusters to find a $\pi^{0}$ candidate and the found photons are then tagged as $\pi^{0}$ photon and excluded from DVCS yield analysis. All the other photons are treated as likely DVCS candidates, but when it comes to the estimate of the DVCS events, only one photon is picked out of all these 9 possible candidates, using the reconstructed missing mass. I pick the one photon that gives a missing mass that is closest to the mass of a proton out of all candidates. Accidental background estimation employs the exact same procedure including the $\pi^{0}$ photon tagging. Figure 52 shows the number of clusters per event and the photon energy distribution. The right plot shows the selected DVCS photons are both independent of the clustering threshold and the photon energy cut. This method, eliminates the systematic due to the cluster threshold and the photon energy cut and make the missing mass cut the only source of systematic. 


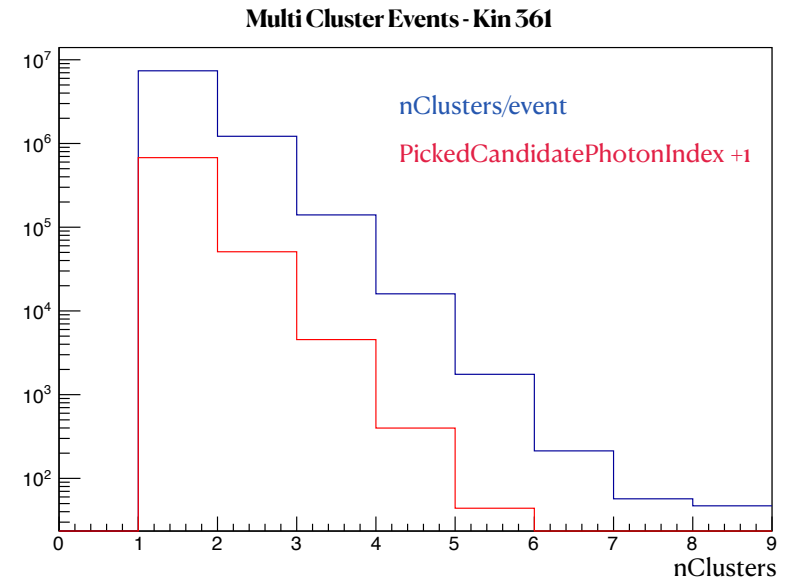

(a) Calorimeter Multi Cluster

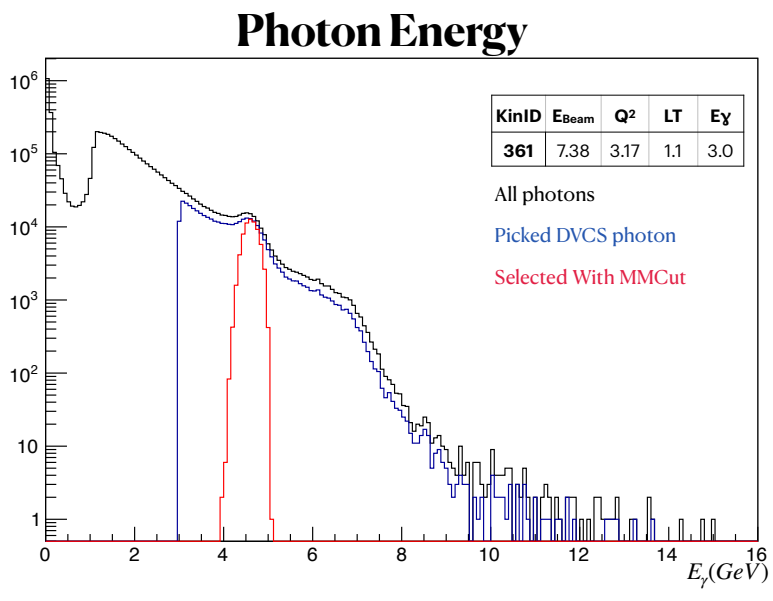

(b) Photon Selection

FIG. 52: Number of clusters recorded in each events are shown in Blue on the left plot. The selected photon Index is shown in Red. The algorithm not always picks the first photon, If we only kept 1 photon in the clustering, either the cases in which the second or higher photon is picked would have been excluded or a photon with a lower likelihood of being from DVCS event would have been picked. Plot on the right shows the photon energy spectrum, Black is for all photon in the true coincidence time window of $[-3,3] n s$, Blue is after the application of Calorimeter fiducial cut discussed in section 6.5. Red spectrum is after applying the missing mass cut shown in Figure 49 . 


\section{CHAPTER 7}

\section{DVCS CROSS SECTION ANALYSIS}

We mentioned in section 1.3 that the first step of the analysis process is to count the number of exclusive DVCS events found in the experiment. This is done by identifying all the final state particle of the reaction $e p \rightarrow e p \gamma$. Electrons are detected in the High Resolution Spectrometer and the selection cuts for identifying a good electron are described in section 5.1. Photons are detected in the DVCS calorimeter and the selection cuts are described in section 6.5. Even when a coincidence timing selection applied to ensure both the electron and photon selected are originated from the same event, there still could be some background photons from accidental coincidences and/or asymmetric $\pi^{0}$ decay. These contaminations are then subtracted from the selected photons as discussed in section 6.6. We apply a missing mass cut discussed in section 6.9.1 to ensure the exclusivity of the process.

The yield of an experiment process is connected to it's cross-section by the following relationship.

$$
N=\mathcal{L} \Delta \Omega \times \frac{d \sigma}{d \Omega}
$$

$\Delta \Omega$ is the space-space from which the accepted events are originated.

$$
\Delta \Omega=\sum_{\text {events }} \frac{\Gamma_{\text {event }}^{D V C S}}{N_{\text {gen }}}
$$

$\Gamma_{\text {event }}^{D V C S}$ given in equation 98 is obtain from the Monte-Carlo simulation described in section 6.7. $N_{\text {gen }}$ is the total number of generated events. $\mathcal{L}$ is the integrated luminosity computed as described in equation 62. The only difference being that the total accumulated charge is computed for the entire kinematic rather than per run as described in section 5.3 . Table 19 tabulates the live time corrected total accumulated charge in coulomb.

\subsection{EXPERIMENTAL EFFICIENCY CORRECTIONS}

Detector inefficiencies and certain selection process will cause us to miss or discard good events. The raw yield obtained should then be corrected for the inefficiencies of the detectors. Let $\eta_{D V C S}$ be the total correction. Then,

$$
\begin{aligned}
N^{\text {exp }} & =N^{\text {selected }} \times \frac{1}{\eta_{D V C S}^{\text {Tot }}} \\
\eta_{D V C S}^{\text {Tot }} & =\eta_{\text {Tracking }} \times \eta_{\text {Trigger_S2 }} \times \eta_{\text {Trigger_CER }} \times \eta_{\text {Radiative }}^{D V C S}
\end{aligned}
$$




\begin{tabular}{|c|c|c|c|c|}
\hline Kin & LTCF & Adjusted $Q^{\text {Total }}(C)$ & $\eta_{\text {Polarization }}$ & $\eta_{\text {hel }}$ \\
\hline $36 \_1$ & 0.965 & 1.8955 & 0.846 & 0.994 \\
\hline $36 \_2$ & 0.968 & 1.63791 & 0.868 & 0.985 \\
\hline $36 \_3$ & 0.949 & 1.24659 & 0.854 & 0.985 \\
\hline $48 \_1$ & 0.984 & 2.17742 & 0.867 & 0.985 \\
\hline $48 \_2$ & 0.952 & 2.08707 & 0.870 & 0.985 \\
\hline $48 \_3$ & 0.977 & 3.63362 & 0.870 & 0.9964 \\
\hline $48 \_4$ & 0.974 & 5.66845 & 0.875 & 0.985 \\
\hline $60 \_1$ & 0.979 & 5.89503 & 0.862 & 0.985 \\
\hline $60 \_3$ & 0.974 & 18.38649 & 0.842 & 0.985 \\
\hline
\end{tabular}

TABLE 19: This table summarizes the average live time correction factors and live time adjusted total accumulated charge. We compute the charge and LTCF run by run and sum them together to get the total accumulated charge $\left(\right.$ Adjusted $\left.Q^{\text {Total }}\right)$. Since the live time correction is already included in the listed charge, we do not apply a correction for it again. Charge calculation is described in section 5.3 and the deadtime corrections are discussed in section 5.4.1. $\eta_{\text {Polarization }}$ is described in section 7.1.1 and $\eta_{\text {hel }}$ in section 7.1.2

$\eta_{D V C S}^{T o t}$ is the total correction. $\eta_{\text {Tracking }}, \eta_{\text {Trigger_S2 }}$ and $\eta_{\text {Trigger_CER }}$ are discussed in section 5.4 and summarized in Table 14. $\eta_{\text {Trigger_S2 }}$ for Spring 2016 kinematics are assumed to be similar to the Fall 2016 runs as discussed in section 4.6. $\eta_{\text {Radiative }}^{D V C S}$ is discussed in section 7.1.3. Dead time corrections for DVCS are computed using a different set of scalars than the DIS computations in Table 14 and included in the charge extraction itself. A summary of average DVCS live time correction factors per kinematic is given in Table 19.

We measure two cross-sections, unpolarized and helicity dependent discussed in section 7.2.3. Two additional corrections $\eta_{\text {Polarization }}$ and $\eta_{\text {hel }}$ applies to the helicity dependent cross section measurements as discussed in next two sub sections.

\subsubsection{POLARIZATION MEASUREMENT}

DVCS3 experiment uses Moller and Compton polarimetry for beam polarization measurements as mentioned in section 3.3.1. We use the Moller results provided by S. Glamazdin and R. Pomatsalyuk [37] in our analysis. For the entire DVCS run period, beam polarization was $\sim 86 \%$ with a statistical uncertainty of $\leq 0.3 \%$ and a systematic uncertainty of 1\%. Table 19 summarizes the polarization measurement for each kinematic settings. The Compton results were consistent with the Moller measurements [24]. 
We apply the polarization as a correction to the helicity dependent yield as $\eta_{D V C S}^{\mathrm{Hel}}=$ $\eta_{D V C S}^{\text {Tot }} \times \eta_{\text {Polarization. }} \eta_{D V C S}^{\text {Tot }}$ is given in equation 102 .

\subsubsection{BEAM HELICITY MEASUREMENT}

Helicity of the beam is flipped at a frequency of $30 \mathrm{~Hz}$ as discussed in section 3.1, and the helicity state is recorded in the data stream. There is a transition window between each helicity flips that results in an uncertainty of beam helicity. Two EPICS variables control the duration in which the helicity state is defined (Helicity Stable Read) and when the helicity state is undefined (Helicity Settle Read), by using the Pockels cell's stable frequency and the transition time. The transition time for the Pockels Cell is $60 \mu s$, but a conservative estimate of $200 \mu s$ for fall 2014 and $500 \mu s$ for later was set. $\eta_{h e l}$ is estimated using equation 103 [70, and used to estimate the helicity dependent charge using equation 104, with the assumption of negligible charge asymmetry.

$$
\begin{array}{r}
\eta_{\text {hel }}=\frac{\text { Helicity Stable Read }}{\text { Helicity Stable Read }+ \text { Helicity Settle Read }} \\
\text { Adjusted } Q^{\text {hel }}=\frac{1}{2} \times \text { Adjusted } Q^{\text {Total }} \times \eta_{\text {hel }}
\end{array}
$$

Estimated $\eta_{\text {hel }}$ and Adjusted $Q^{\text {Total }}$ are given in Table 19 .

\subsubsection{INTERNAL RADIATIVE CORRECTIONS - DVCS}

There are additional real and virtual radiations occur at the vertex contributing to radiation loss called internal radiation are discussed in section 5.5.1. Figure 53 illustrates the different internal radiative processes for DVCS.
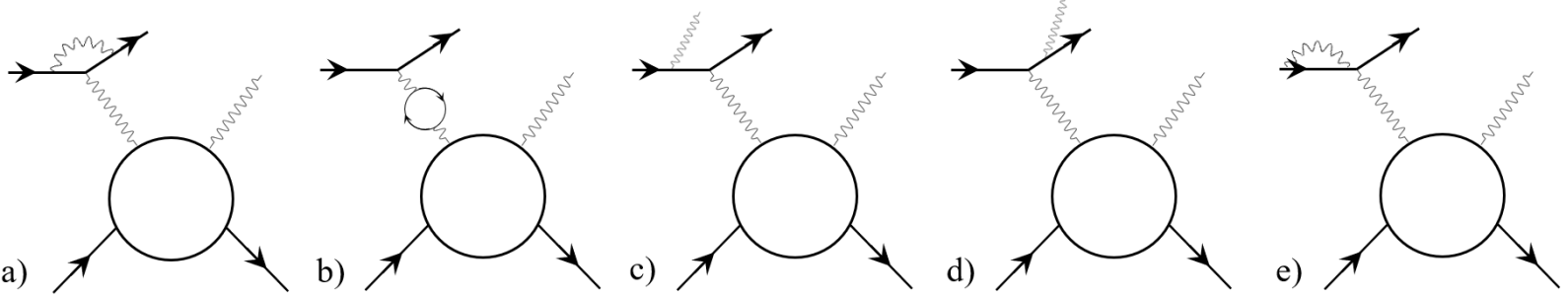

FIG. 53: The diagrams shows the different internal radiative processes for DVCS

Internal radiative correction discussed by $\mathrm{M}$. Vanderhaeghen et al [79] is adapted to Hall-A DVCS exclusive process by Charles E. Hyde [44] and given as,

$$
\left.\frac{d \sigma}{d \Omega}\right|_{\text {exp }}=\left.\frac{d \sigma}{d \Omega}\right|_{b o r n}\left[\frac{e^{\delta_{\text {vertex }}+\delta_{R}^{0}}}{\left(1-\delta_{\text {vac }}\right)^{2}}\right]\left(\frac{\Delta E}{\sqrt{E E^{\prime}}}\right)^{\delta_{s}}
$$


here,

$$
\begin{aligned}
\delta_{s} & =\frac{2 \alpha}{\pi}\left[\ln \left(\frac{Q^{2}}{m_{e}^{2}}\right)-1\right] \\
\delta_{\text {vertex }} & =\frac{\alpha}{\pi}\left[\frac{3}{2} \ln \left(\frac{Q^{2}}{m_{e}^{2}}\right)-2-\frac{1}{2} \ln ^{2} \ln \left(\frac{Q^{2}}{m_{e}^{2}}\right)+\frac{\pi^{2}}{6}\right] \\
\delta_{v a c} & =\frac{\alpha}{3 \pi}\left[\ln \left(\frac{Q^{2}}{m_{e}^{2}}\right)-\frac{5}{3}\right] \\
\delta_{R}^{0} & =\frac{\alpha}{\pi}\left[\frac{1}{2} \ln ^{2}\left(\frac{Q^{2}}{m_{e}^{2}}\right)-\frac{1}{2} \ln ^{2} \ln \left(\frac{E}{E^{\prime}}\right)+\frac{\pi^{2}}{3}+S_{p}\left(\cos ^{2}\left(\frac{\theta_{e}}{2}\right)\right)\right]
\end{aligned}
$$

The Spence function $S p(z)$ is defined as

$$
S p(z)=-\int_{0}^{z} \frac{\ln (1-t)}{t} d t
$$

Equation 105 is an approximate formula based on elastic scattering. $\left(\frac{\Delta E}{\sqrt{E E^{\prime}}}\right)^{\delta_{s}}$ term is incorporated in to the Monte-Carlo discussed in section 6.7. $\left[\frac{e^{\delta_{\text {vertex }}+\delta_{R}^{0}}}{\left(1-\delta_{\text {vac }}\right)^{2}}\right]$ term in all kinematics are computed by Charles E. Hyde and given in [45] using codes developed by D. Lhuillier et. al. [25].

\subsection{CROSS SECTION EXTRACTION METHOD}

Belitsky-Muller-Kirchner [BMK] model [14] provides the harmonic expansion of the amplitude terms of the DVCS cross section. Angular harmonics can be expressed as a product of a pure kinematic term and t-dependent linear and bilinear combinations of Elastic Form factors $F_{1}$ and $F_{2}$ and Compton Form Factors (CFFs). To handle the t- dependency of the angular harmonic terms, we bin our phase-space. Then the cross section parametrization discussed in section 2.4 .2 can be expressed as follows,

$$
\begin{array}{r}
\left\langle\frac{d \sigma}{d \Omega}\right\rangle_{B i n}=\sum_{j}^{n C F F} \sum_{B i n}^{n B i n} K_{j, B i n}\left(E, Q^{2}, x_{B}, t, \phi_{\gamma \gamma}, \phi_{e}\right) \times C_{j, B i n}(t) \\
\text { where; } C j \in\left\{C_{n}^{T}, S_{n}^{T} \mid T=B H, D V C S, \mathcal{I} ; n=0 \ldots 3\right\}
\end{array}
$$

The experimental yield in a given bin, then can be related to the cross section by the following relationship.

$$
N_{\text {Bin }}^{\text {exp }}=\sum_{i \in \text { events }} \frac{\mathcal{L} \Gamma_{i}}{N_{\text {gen }}}\left\langle\frac{d \sigma}{d \Omega}\right\rangle_{\text {Bin }}
$$


$\Gamma_{i}$ computation is given in equation 98. Equations 112 and 113 will give the following with a minor caveat discussed in next section,

$$
N_{\text {Bin }}^{\text {exp }}=\sum_{i \in \text { events }} \frac{\mathcal{L} \Gamma_{i}}{N_{g e n}}\left(\sum_{j}^{n C F F} \sum_{\text {Bin }}^{n B i n}\left(K_{j, B i n}\left(E, Q^{2}, x_{B}, t, \phi_{\gamma \gamma}, \phi_{e}\right) \times C_{j, \text { Bin }}(t)\right)\right)
$$

\subsubsection{BIN MIGRATION}

If we pay a little closer attention to the equation 114, we will realize that, the Bin indices on the left side of the equations are for the detected variables and the right side are for the vertex variables. In other words the event distribution happens according to the kinematics at which it originated and the experimental yield is extracted at the kinematics where the events are landed after all the radiation and resolution effects. This means that an event originating at one bin can migrate to another bin at the detector. This is called bin migration. We have access only to the detector variables in our experiment. We construct the vertex variables from the geant 4 simulation discussed in section 6.7. We need to find a way to map these two bins.

Lets define a migration matrix $\mathcal{M}$ with elements

$$
\mathcal{M}_{d v}= \begin{cases}1, & \text { if the event originating at the vertex bin } v \text { landed in the detector bin } d \\ 0, & \text { otherwise }\end{cases}
$$

now we can re-write the equation 114 with the help of equation 115 as follows,

$$
N_{d}^{e x p}=\mathcal{L} \sum_{j}^{n C F F} \sum_{v}^{n v_{b i n}} \sum_{\text {events }}^{N_{\text {gen }}} \sum_{v} \mathcal{M}_{d v} \frac{\Gamma_{i}}{N_{g e n}} \times K_{j, v}\left(E, Q^{2}, x_{B}, t, \phi_{\gamma \gamma}, \phi_{e}\right) \times C_{j, v}(t)
$$

It is easier to see from the equation 115 that,

$$
\sum_{\text {events }} \sum_{v} \mathcal{M}_{d v}=\sum_{i \in d \cap v}
$$

then we will get,

$$
N_{d}^{e x p}=\mathcal{L} \sum_{j}^{n C F F} \sum_{v}^{n v_{b i n}} \sum_{i \in d \cap v} \frac{\Gamma_{i}}{N_{g e n}} \times K_{j, v}\left(E, Q^{2}, x_{B}, t, \phi_{\gamma \gamma}, \phi_{e}\right) \times C_{j, v}(t)
$$




\subsubsection{CROSS-SECTION FITTING PROCEDURE}

In DVCS3 experiment, we not only want to extract the cross-section, we want to extract the Compton Form Factors. If we look at the equation 118, the right side of it is completely from Monte-Carlo. We can think of it as the Monte-Carlo yield $\left(N^{s i m}\right)$. We can treat the angular harmonic terms $C_{j, v}(t)$ as free parameters and fit the experimental yield to the simulated yield to extract these parameters by minimizing the $\chi^{2}$ given in equation 119. Then equation 112 can be used to construct the experimental cross-section from the extracted angular harmonics.

$$
\chi^{2}=\sum_{d}^{N_{b i n}}\left(\frac{N_{d}^{e x p}-N_{d}^{s i m}}{\sigma_{k}}\right)^{2}
$$

here, $\sigma_{k}$ is the associated error for the extracted experimental yield.

Let,

$$
\mathcal{K}_{j}=\sum_{v}^{n v_{b i n}} \sum_{i \in d \cap v} \frac{\Gamma_{i}}{N_{g e n}} \times K_{j, v}\left(E, Q^{2}, x_{B}, t, \phi_{\gamma \gamma}, \phi_{e}\right)
$$

Then, for the minimization $\frac{\partial \chi^{2}}{\partial C_{j, v}}=0$, we can get the following from equation 118 ,

$$
\begin{aligned}
0 & =\sum_{d}^{N_{b i n}}\left(\frac{N_{d}^{e x p}-\mathcal{L} \sum_{j}^{n C F F} \mathcal{K}_{j} C_{j, v}}{\sigma} \times \frac{1}{\sigma}\left(0-\mathcal{L} \sum_{j}^{n C F F} \mathcal{K}_{j}\right)\right) \\
& =\sum_{d}^{N_{b i n}}\left(\frac{\sum_{j}^{n C F F} \sum_{j^{\prime}}^{n C F F} \mathcal{L}^{2} \mathcal{K}_{j} \mathcal{K}_{j^{\prime}} C_{j, v}}{\sigma^{2}}-\frac{N_{d}^{\exp } \sum_{j}^{n C F F} \mathcal{L} \mathcal{K}_{j}}{\sigma^{2}}\right)
\end{aligned}
$$

Let,

$$
\begin{aligned}
\alpha_{j, j^{\prime}} & =\sum_{d}^{N_{\text {bin }}} \frac{\mathcal{L}^{2} \mathcal{K}_{j} \mathcal{K}_{j^{\prime}}}{\sigma^{2}} \\
\beta_{j} & =\frac{N_{d}^{\exp } \mathcal{L} \mathcal{K}_{j}}{\sigma^{2}}
\end{aligned}
$$

we get the matrix equation,

$$
\alpha C_{j, v}-\beta=0
$$

$C_{j, v}(t)$ are dependent only on $t_{\text {bins }}$ and uniform within the bin. That means, $\alpha$ is a $n C F F$. $n t_{\text {bin }} \times n C F F \cdot n t_{\text {bin }}$ square matrix and $\beta$ is a $n C F F \cdot n t_{\text {bin }}$ column vector.

$$
C_{j, v}=\alpha^{-1} \times \beta
$$


We make 5 bins in $t-t_{\text {min }}$ for experimental data and Monte-Carlo. $t=(p-p)^{2}=\left(q-q^{\prime}\right)^{2}$ is the total 4-momentum transfer squared at the proton vertex. Since, we don't detect the final state proton, we use $q$ and $q^{\prime}$, which are the virtual and real photon 4-momenta to compute $t . t_{\min }$ is given bellow,

$$
t_{\text {min }}=\frac{Q^{2} M_{p}+2 M_{p} \nu(\nu-|\vec{q}|)}{|\vec{q}|-\nu-M_{p}}
$$

here, $\nu$ is the energy transfer at electron vertex, $\vec{q}$ is the virtual photon 3-momentum and $M_{p}$ is proton mass. The data will have an additional 24 bins in $\phi_{\gamma \gamma}$ defined in equation 40 .

\subsubsection{UNPOLARIZED AND HELICITY DEPENDENT CROSS SECTION}

The full form of the parametrization with trivialized helicity dependence can be expressed as,

$$
\frac{d^{4} \sigma}{d \Omega}=\left.\frac{d^{4} \sigma}{d \Omega}\right|_{B H}+\sum_{n=0}^{N} C_{n}(t) \cos (n \phi) K_{n}\left(Q^{2}, x_{B}, t\right)+\sum_{n=0}^{N} \lambda S_{n}(t) \sin (n \phi) \widetilde{K}_{n}\left(Q^{2}, x_{B}, t\right)
$$

here, $\lambda$ is helicity. We can write,

$$
\begin{aligned}
& d \sigma=\frac{\frac{d \sigma+}{d \Omega}+\frac{d \sigma-}{d \Omega}}{2}=\left.\frac{d^{4} \sigma}{d \Omega}\right|_{B H}+\sum_{n=0}^{N} C_{n}(t) \cos (n \phi) K_{n}\left(Q^{2}, x_{B}, t\right) \\
& d \Sigma=\frac{\frac{d \sigma+}{d \Omega}-\frac{d \sigma-}{d \Omega}}{2}=\sum_{n=0}^{N} \lambda S_{n}(t) \sin (n \phi) \widetilde{K}_{n}\left(Q^{2}, x_{B}, t\right)
\end{aligned}
$$

here + or - in the cross section denotes the helicity state of the probe.

Equation 128 is defined as Unpolarized Cross Section and It is sensitive to the $\cos (n \phi)$ terms and equation 129 is defined as Helicity Dependent Cross Section and It is sensitive to the $\sin (n \phi)$ terms.

\subsubsection{CHOICES OF PARAMETERS}

DVCS cross section has many angular harmonic terms. The pure DVCS term has 3 $\cos (n \phi)$ terms with $n=\{0,1,2\}$ and $2 \sin (n \phi)$ terms with $n=\{1,2\}$. The Interference term of the cross section has one additional sin and $\cos$ term for $\mathrm{n}=3$. The angular harmonics themselves are combinations of twist 2 and twist 3 Compton Form Factors. The $\phi$ dependence alone is not sufficient to separate all possible combinations of CFF terms. $\phi$ dependence however, can be fully described by many choices of parametrization while the extraction of the cross-section is independent of the choices of parametrization. 
I make two different sets of choices for the parametrization. The expressions for the Choice 1 is given in [12] and Choice 2 is given in [13]. The physical interpretation of the choices in terms of the Compton Form Factor combinations are stated in the following two sub sections.

\section{Choice1 : $D V C S^{2}$ Terms Only}

$$
\begin{gathered}
C_{\text {unp }}^{V C S}\left(\mathcal{F}_{++}, \mathcal{F}_{++}^{*} \mid \mathcal{F}_{-+}, \mathcal{F}_{-+}^{*}\right) \\
\left\{\begin{array}{l}
\operatorname{Re} \\
\operatorname{Im}
\end{array}\right\} C_{\text {unp }}^{V C S}\left(\mathcal{F}_{0+} \mid \mathcal{F}_{++}^{*}, \mathcal{F}_{-+}^{*}\right) \\
\left\{\begin{array}{l}
\operatorname{Re} \\
\operatorname{Im}
\end{array}\right\} C_{\text {unp }}^{V C S}\left(\mathcal{F}_{-+}, \mathcal{F}_{++}^{*}\right)
\end{gathered}
$$

\section{Choice2 : $C_{0}^{D V C S^{2}}$ and Interference terms}

$$
\begin{aligned}
C_{\text {unp }}^{V C S} & \left(\mathcal{F}_{++}, \mathcal{F}_{++}^{*} \mid \mathcal{F}_{-+}, \mathcal{F}_{-+}^{*}\right) \\
& \left\{\begin{array}{l}
\operatorname{Re} \\
\operatorname{Im}
\end{array}\right\}\left\{\begin{array}{c}
C_{++}^{\mathcal{I}}\left(1 \mid \mathcal{F}_{++}\right) \\
S_{++}^{\mathcal{I}}\left(1 \mid \mathcal{F}_{++}\right.
\end{array}\right\}_{\text {unp }} \\
& \left\{\begin{array}{l}
\operatorname{Re} \\
\operatorname{Im}
\end{array}\right\}\left\{\begin{array}{c}
C_{0+}^{\mathcal{I}}\left(2 \mid \mathcal{F}_{0+}\right) \\
S_{0+}^{\mathcal{I}}\left(2 \mid \mathcal{F}_{0+}\right.
\end{array}\right\}_{\text {unp }}
\end{aligned}
$$

\subsubsection{FITTED YIELD}

Figure 54 shows the fitted yield (Unpolarized) for kin 362 in all $5 t^{\prime}$ bins using both parametrization choices given in section 7.2.4. Overall reduced $\chi^{2}$ for this fit is 1.66 and 1.37 for choice 1 and 2. At the edges of the tbins, where $\phi=0^{0}$ or $360^{\circ}$, the acceptance drops and the fit becomes slightly unreliable in many tBins. The last $t_{b i n}$ is only used for bin migration. The events in this bin can migrate out, but there is no bin outside of it to migrate in, and hence the fit and cross section are expected to be poorly reconstructed in this bin.

\subsection{PRELIMINARY RESULTS}

Angular harmonic terms $C_{j}, v$ extracted from equation 125 by fitting the experimental yield to the simulated yield as shown in Figure54 together with the kinematic terms $K_{j}$, bin whose forms are given in [12, 13], computed at the center of the bin are used to compute the cross section from equation 112 . Figure 55 shows the extracted unpolarized cross section for 

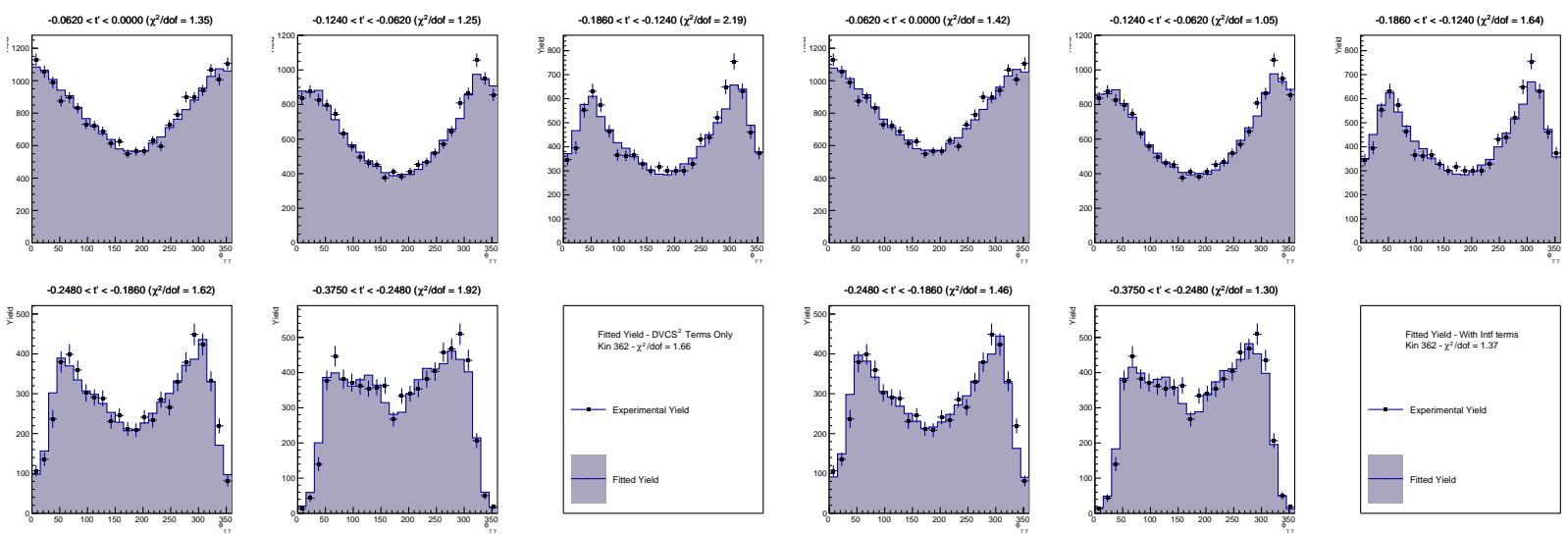

(a) DVCS Only Fit

(b) Fit with $\mathcal{I}$

FIG. 54: Fitted yield results for Kin 362 unpolarized case is shown here. Reduced $\chi^{2}$ for the overall fit is 1.66 for choice 1 and 1.37 for choice 2. The points are the experimental yield and the error bars are statistical only. Fit is given as the fill. Parametrization Choices are given in section 7.2 .4

kin 362 at $t_{\text {Bin }}-0.062<t^{\prime}<0.000$ with Beam energy $=8.521 \mathrm{GeV},<Q^{2}>=3.6 \mathrm{GeV}^{2}$ and $\left\langle x_{B}\right\rangle=0.36$. Figure 56 shows the helicity dependent cross section for kin 361 at $t_{B i n}$ $-0.087<t^{\prime}<0.000$ with Beam energy $=7.38 \mathrm{GeV},<Q^{2}>=3.2 \mathrm{GeV}^{2}$ and $<x_{B}>=0.36$. Unpolarized cross sections are sensitive to Bethe-Heitler and $\cos (n \phi)$ terms of the angular harmonics. Helicity dependent cross section is sensitive to the $\sin (n \phi)$ terms of the angular harmonics.

We extracted cross section with two different parametrization choices. The agreement in the extracted cross section between the two parametrization choices validates that the extracted cross sections are in fact independent of the choices of parametrization while the independent angular harmonics extracted are a function of the choice. Figure 57 shows a comparison between the two choices. This is a particularly interesting validation since we directly fit the free parameters rather than extracting a cross section in the traditional way. The two choices are discussed in section 7.2.4.

Both Unpolarized and Helicity dependent cross sections are compared against two main global fits to the DVCS data KM10a and KM15 given in [57] and [56]. KM15 includes data from previous Hall-A experiments while the KM10a doesn't. The model cross sections are computed using the the executable available from http://calculon.phy.hr/gpd/. The site links to the executable that used for the low $\mathrm{x}$ data. For our kinematics we used the executable available through the download link http://calculon.phy.hr/gpd/xs $s_{l}$ argex.exe. 


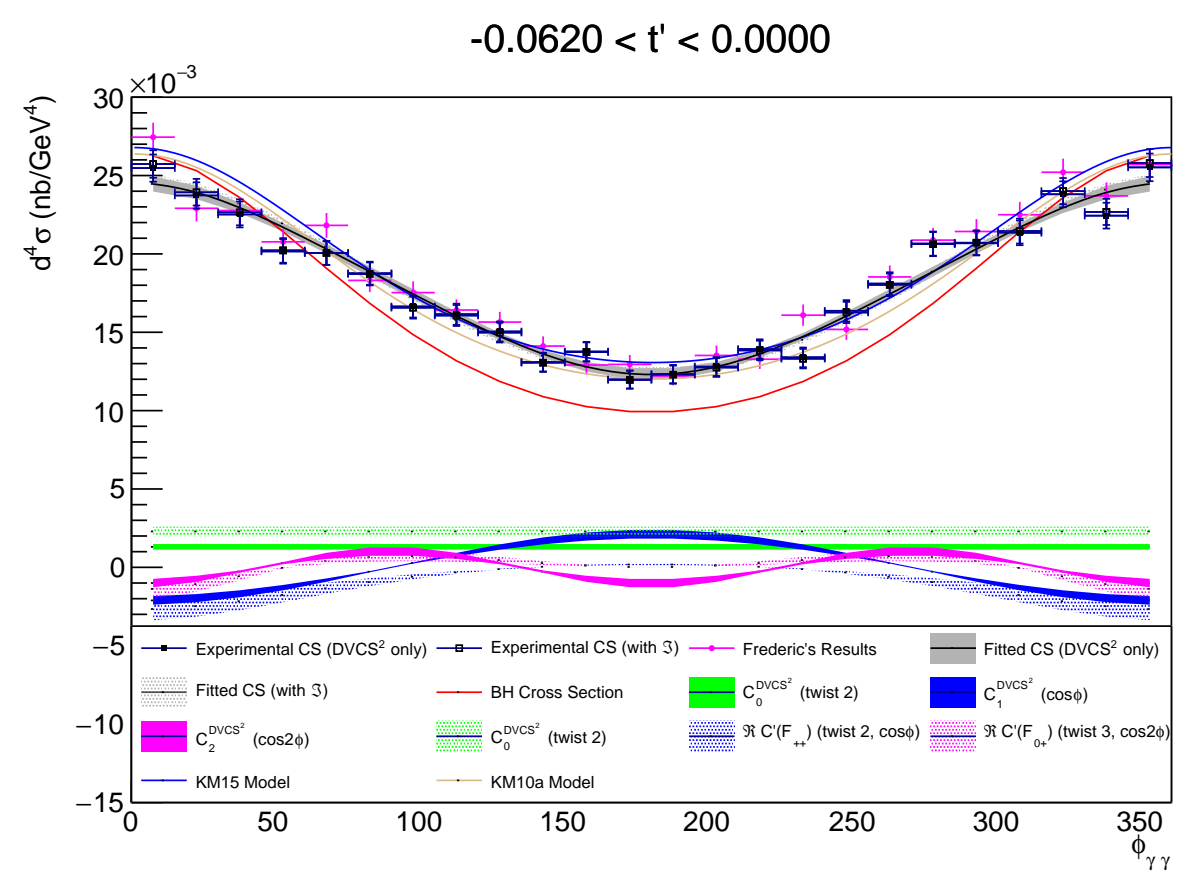

FIG. 55: DVCS Unpolarized cross section for kin 362 at $t_{\text {Bin }}-0.062<t^{\prime}<0.000$ with Beam energy $=8.521 \mathrm{GeV},\left\langle Q^{2}\right\rangle=3.6 \mathrm{GeV}^{2}$ and $\left\langle x_{B}\right\rangle=0.36$. Closed boxes $/$ Filled colors represents Choice 1 given in section 7.2.4. The open boxes/ Polka Squares represents Choice 2 given is section 7.2.4. Red curve is the contribution from pure BH cross section. The grey bands around the fits are the statistical uncertainty. Magenta dots represents a parallel analysis by Frédéric Georges [33]. Blue and Brown lines are two global fits given in [57] [56]. The agreement between the different parametrization validates the claim that the cross section is independent of the choices of parametrization while the extracted terms are dependent on the choice. The bands structures closer to the bottom in the plots are the individual angular harmonics. This analysis and the parallel analysis agrees within error bars. KM15 model represents our data better than KM10 model. 


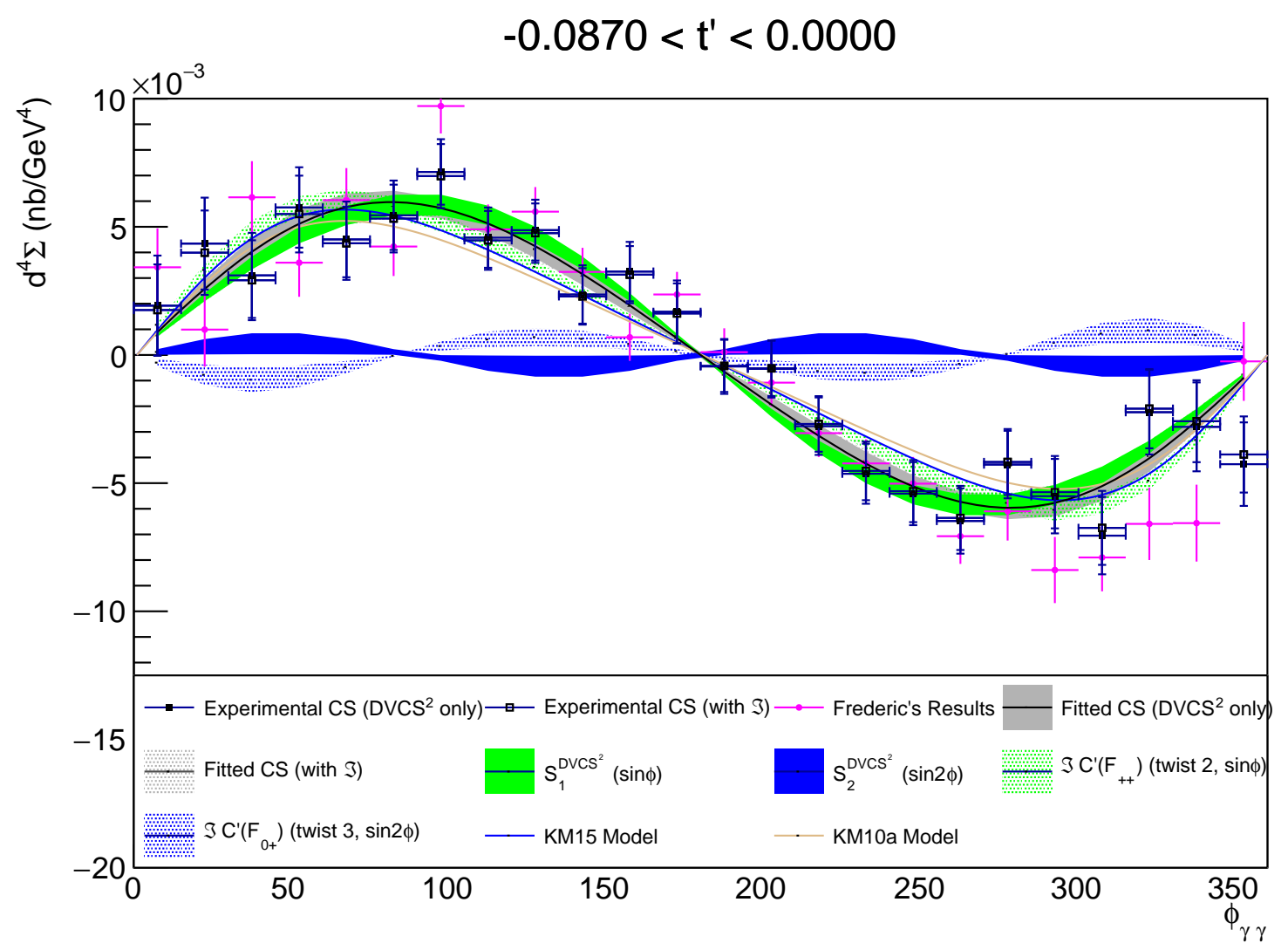

FIG. 56: DVCS helicity dependent cross section for kin 361 with Beam energy $=7.38 \mathrm{GeV}$, $\left.<Q^{2}\right\rangle=3.2 \mathrm{GeV}^{2}$ and $\left\langle x_{B}\right\rangle=0.36$. Closed boxes/Filled colors represents Choice 1 given in section 7.2.4. Open boxes/ Polka Squares represents Choice 2 given in section 7.2.4. The Grey bands around the fits are the statistical uncertainty. Magenta dot represents a parallel analysis by Frédéric Georges [33]. Blue and Brown lines are two global fits given in [57, 56]. Agreement between the different parametrization validates the claim that the cross section is independent of the choices of parametrization. This analysis and the parallel analysis agrees to the most part. There are slight disagreement in the edges of the bin at $\phi=0^{0}$ or $\phi=360^{\circ}$. The Blue and Green bands are the individual angular harmonic terms. 

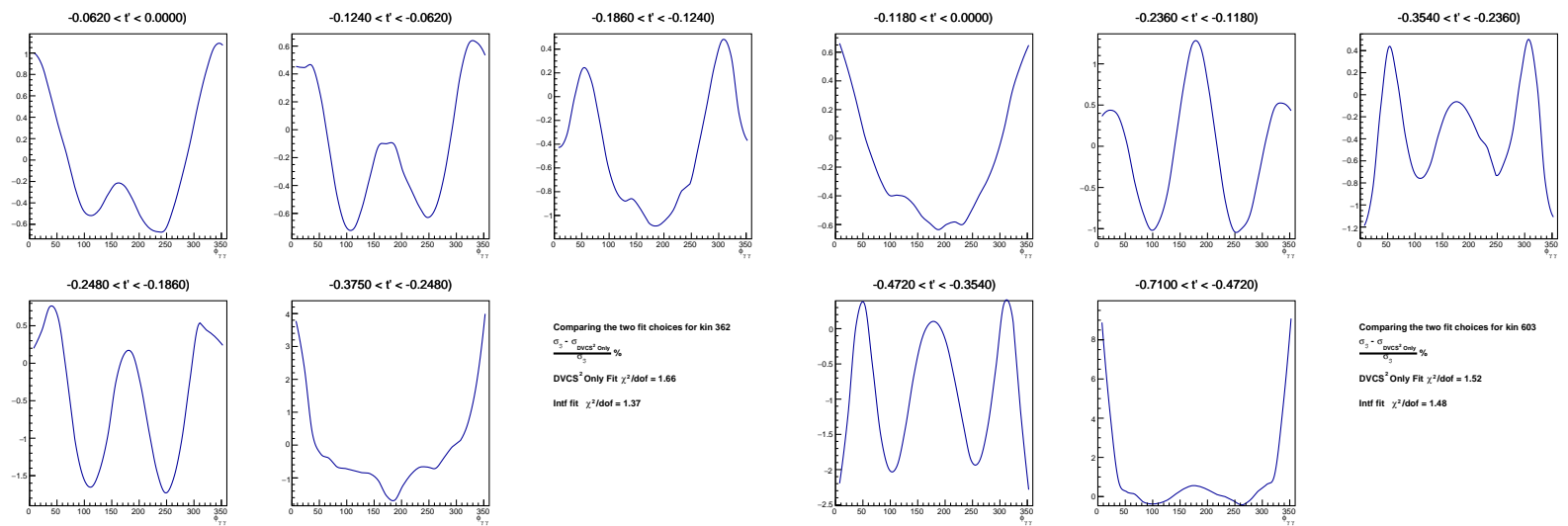

(a) Kin 362

(b) Kin 603

FIG. 57: Figure shows a comparison between two different choices of parametrization discussed in section 7.2.4. Here I plot $\frac{\sigma_{w i t h \mathcal{I}}-\sigma_{D V C S_{\text {only }}^{2}}^{2}}{\sigma_{\text {withI }}} \%$ in each bin of kin 362 and 602 . The uncertainty is within $1 \%$ for most bin. The angular structure is coming from the angular dependency of the Bethe-Heitler propagators in the denominator of the Interference terms.

Model computation for the large $t_{B i n}$ 's of the kinematic 601 and 603 are still not available through the executable. A prevailing feature is that the model KM15 better describes our unpolarized data than the KM10a in most kinematics. When $x_{B}$ gets higher the model starts to deviate from the data, but this is expected as not enough word data is available in these high $x_{B}$ values to tune the model better. The KM10a model most often underestimates the cross section.

I compare my results with a parallel analysis by Frédéric Georges given in [33]. For most part the results are in agreement within error bars as shown in Figure 58. The results differ considerably in the first two $t_{\text {Bin }}$ of kin 601 (Figure 73) and 603 (Figure 75). Interestingly KM10a is closer to my results in kin 603 and KM15 is closer to Frédéric Georges'. This slight difference could be due to a change in the R-Function between the two analysis. DAQ live time correction factors used in Frédéric Georges' analysis are estimated using the DIS scalars and equal to the values given in Table 14 . The values used in this analysis are computed using the DVCS scalars and given in Table 19 . This change accounts for the minor variations seen in few of the kin 48 bins together with the R-Function update. 

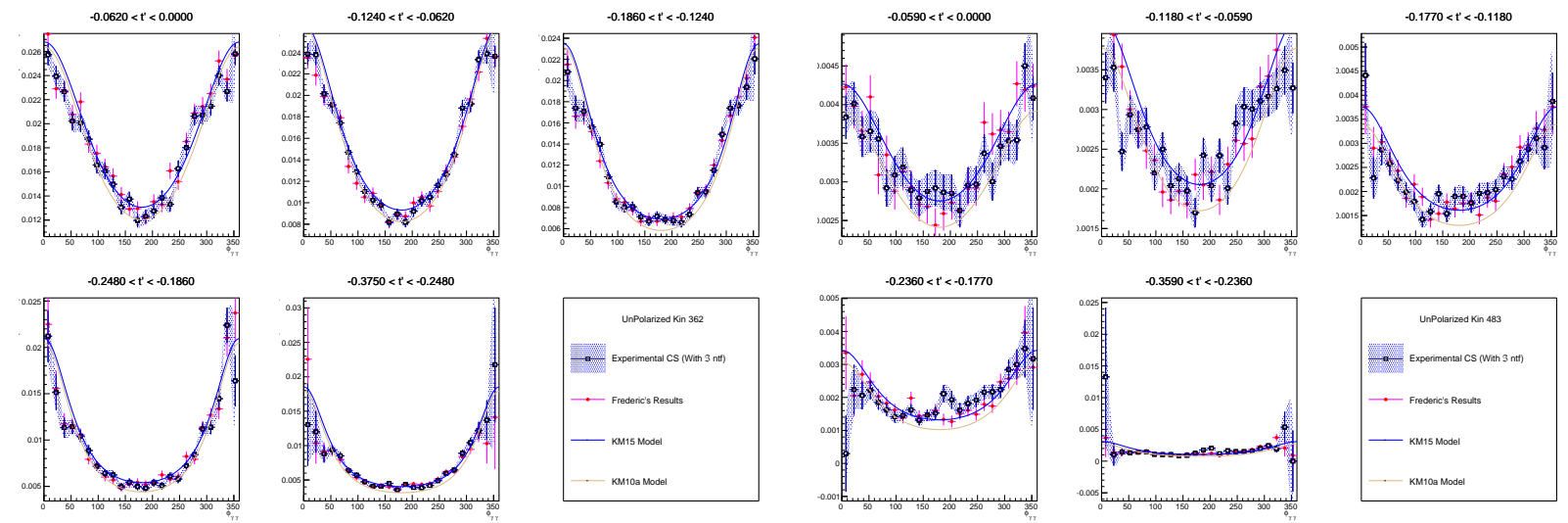

(a) Kin 362

(b) Kin 483

FIG. 58: Figure shows a comparison between two parallel cross section extraction performed in this thesis and by Frédéric Georges in [33. The two extractions are in agreement within the error bar except for the first $2 t_{\text {bins }}$ of kin 601 and 603 as discussed in the text.

Cross Section results for All 9 kinematics in all $5 t_{b i n}$ are given in Figure 59 to Figure 76. The last $t_{b i n}$ is there to estimate the events migrating into the bin before it. Since there is no bin next to it for the events to migrate from, the cross sections in the last $t_{b i n}$ are unreliable. Acceptance drops at the edges of the bin around $\phi=0^{0}$ or $\phi=360^{\circ}$ and the statistical uncertainty raises as a result. 

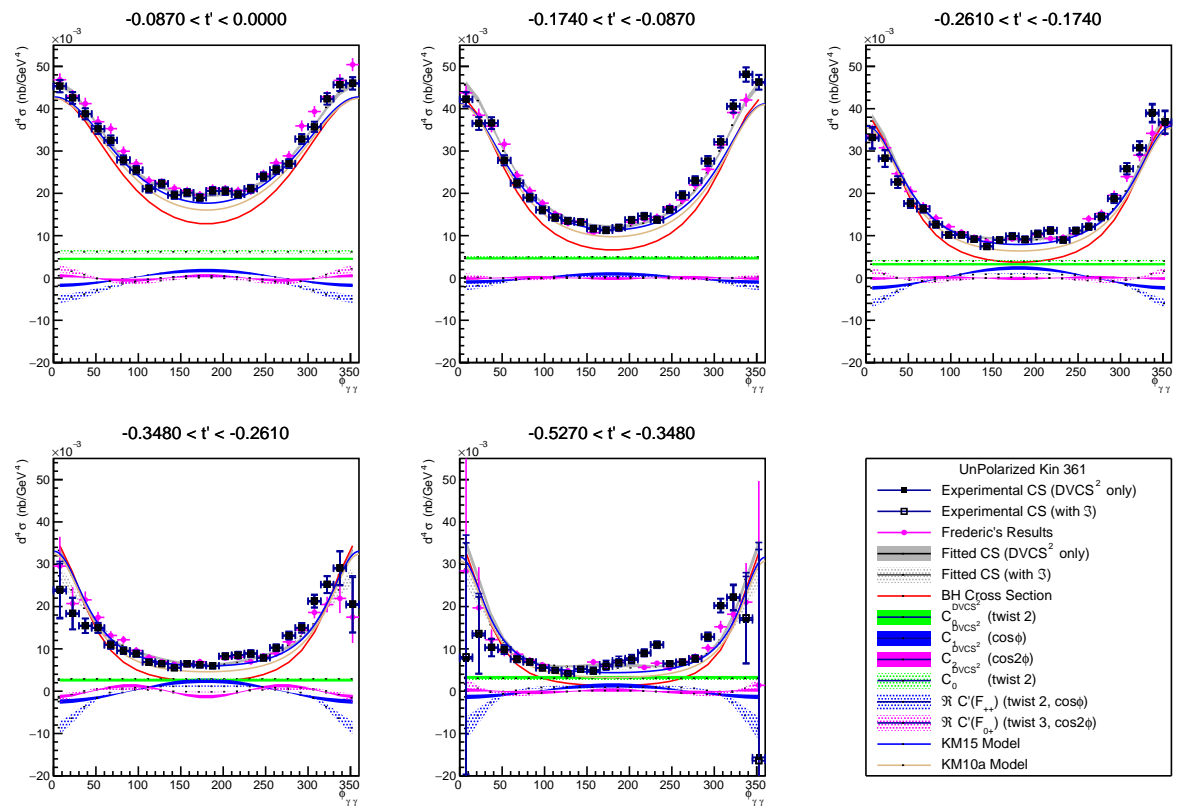

FIG. 59: DVCS unpolarized cross section for Kin 361. Cross section is extracted with 2 different parametrization choices discussed in section 7.2.4. The bands around the fits are the statistical uncertainty. Magenta dot represents a parallel analysis by Frédéric Georges [33].
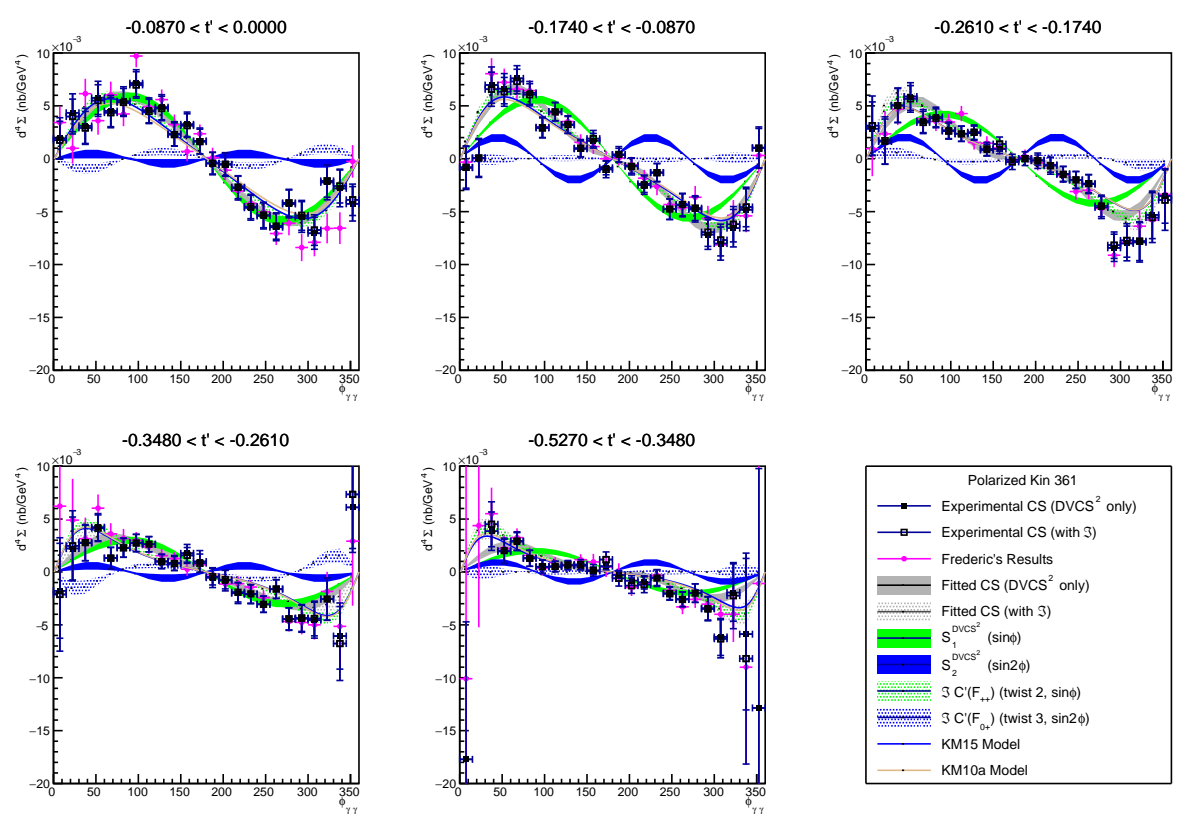

FIG. 60: DVCS helicity dependent cross section for Kin 361. Cross section is extracted with 2 different parametrization choices discussed in section 7.2.4. The bands around the fits are the statistical uncertainty. Magenta dot represents a parallel analysis by Frédéric Georges[33]. 

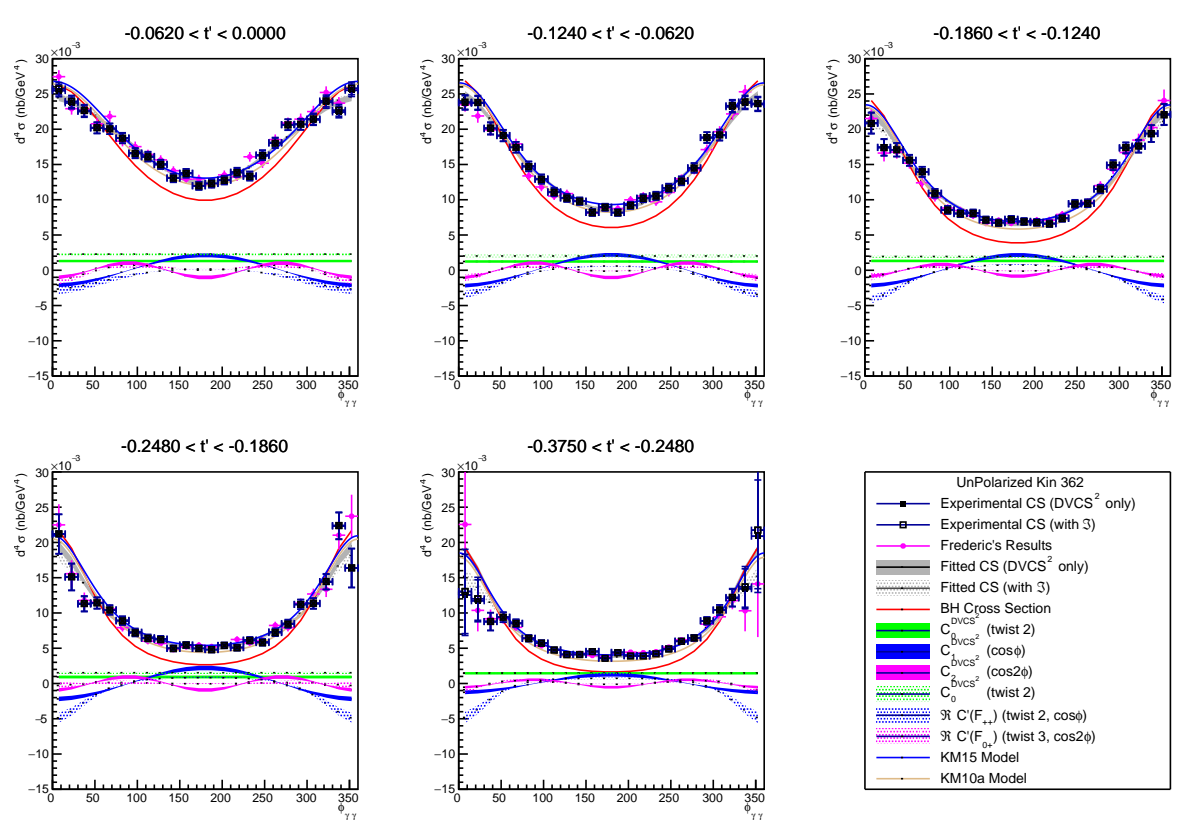

FIG. 61: DVCS unpolarized cross section for Kin 362. Cross section is extracted with 2 different parametrization choices discussed in section 7.2.4. The bands around the fits are the statistical uncertainty. Magenta dot represents a parallel analysis by Frédéric Georges [33]. Model cross sections are given in [57 [56]
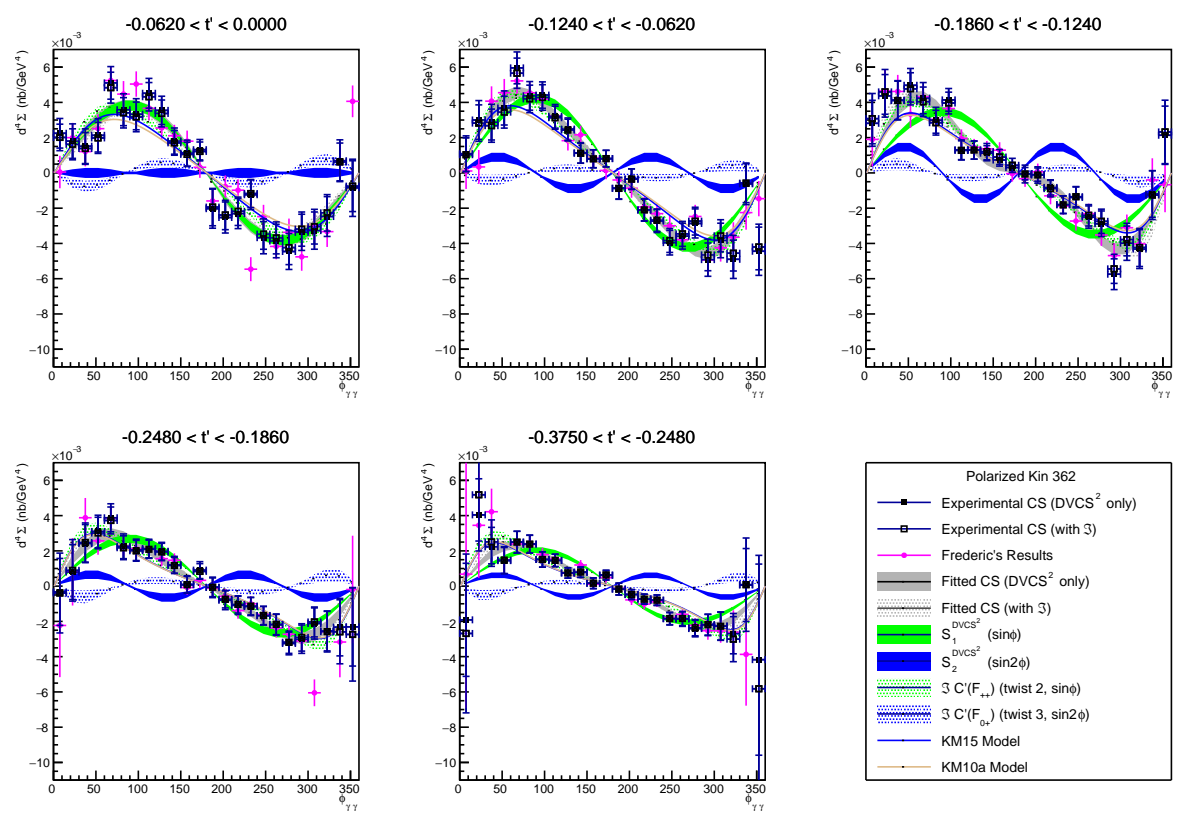

FIG. 62: DVCS helicity dependent cross section for Kin 362. Cross section is extracted with 2 different parametrization choices discussed in section 7.2.4. The bands around the fits are the statistical uncertainty. Magenta dot represents a parallel analysis by Frédéric Georges [33]. Model cross sections are given in [57] [56] 

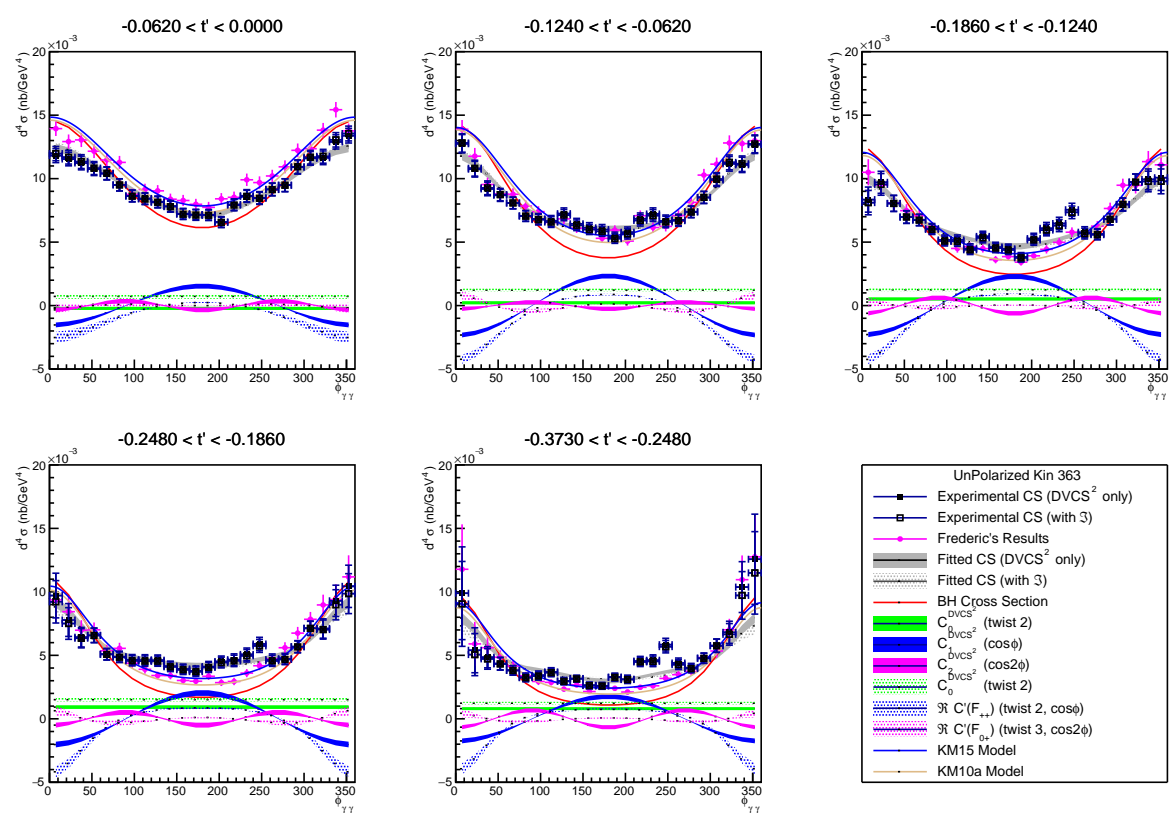

FIG. 63: DVCS unpolarized cross section for Kin 363. Cross section is extracted with 2 different parametrization choices discussed in section 7.2.4. The bands around the fits are the statistical uncertainty. Magenta dot represents a parallel analysis by Frédéric Georges [33]. Model cross sections are given in [57] [56]
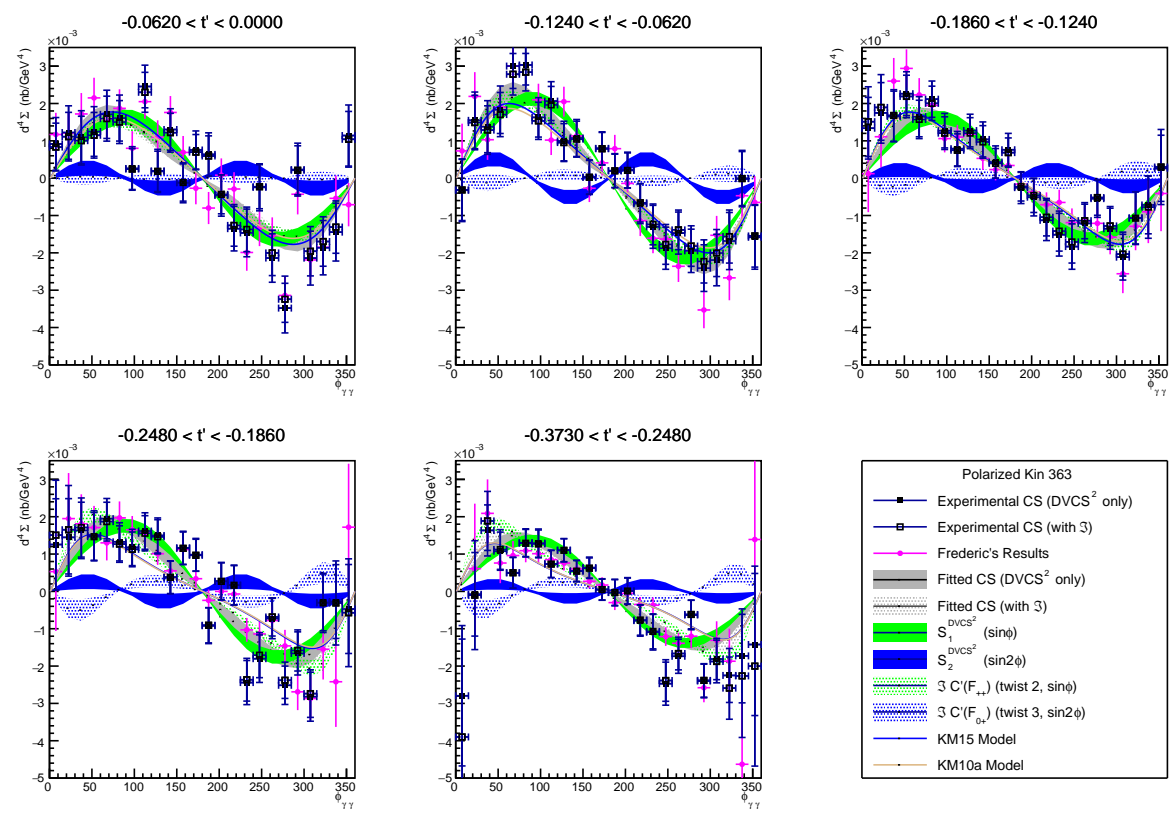

FIG. 64: DVCS helicity dependent cross section for Kin 363. Cross section is extracted with 2 different parametrization choices discussed in section 7.2.4. The bands around the fits are the statistical uncertainty. Magenta dot represents a parallel analysis by Frédéric Georges [33]. Model cross sections are given in [57] [56] 

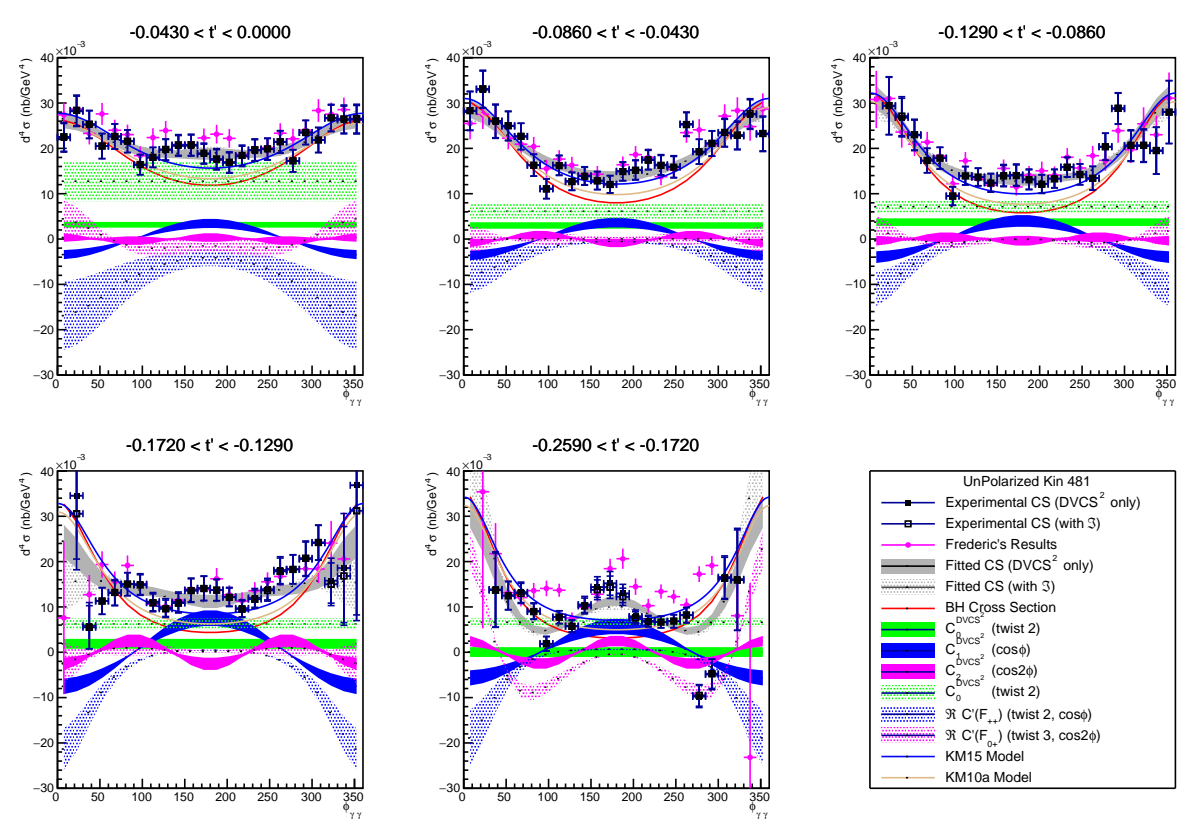

FIG. 65: DVCS unpolarized cross section for Kin 481. Cross section is extracted with 2 different parametrization choices discussed in section 7.2.4. The bands around the fits are the statistical uncertainty. Magenta dot represents a parallel analysis by Frédéric Georges [33]. Model cross sections are given in [57] [56]
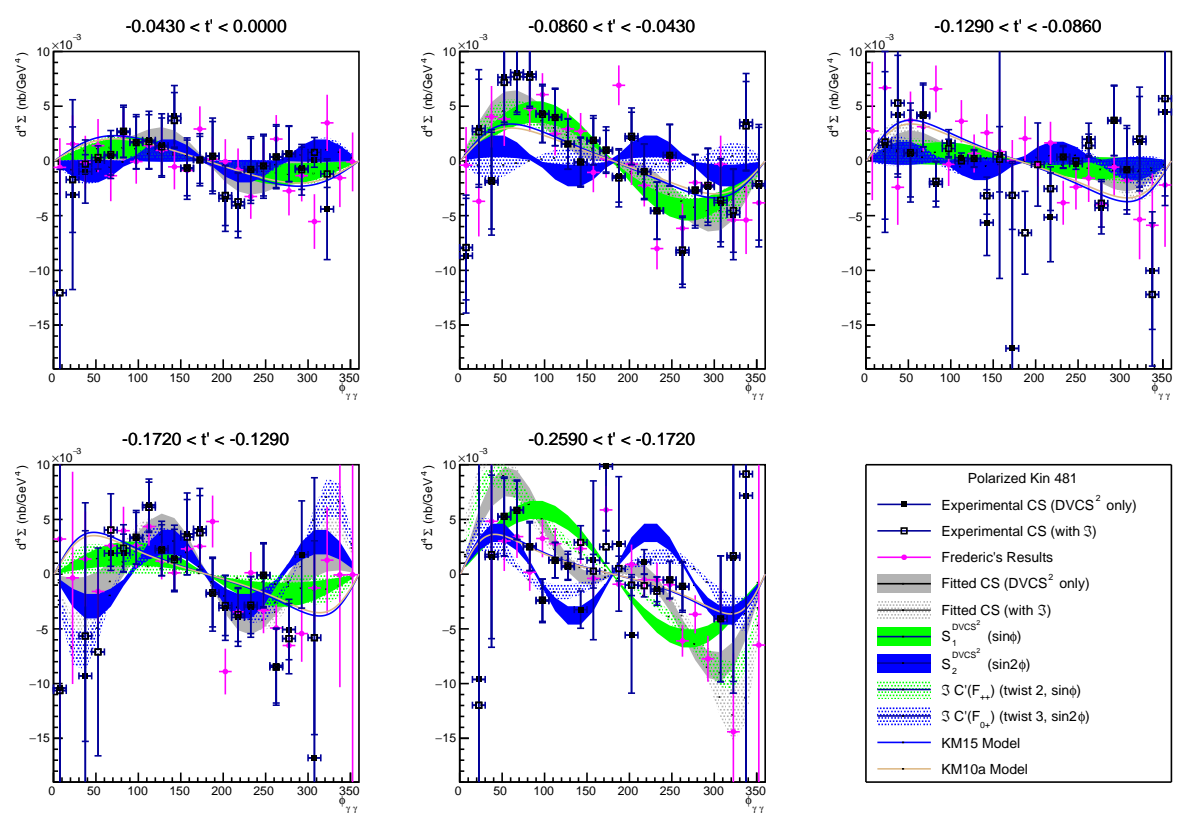

FIG. 66: DVCS helicity dependent cross section for Kin 481. Cross section is extracted with 2 different parametrization choices discussed in section 7.2.4. The bands around the fits are the statistical uncertainty. Magenta dot represents a parallel analysis by Frédéric Georges [33]. Model cross sections are given in [57] [56] 

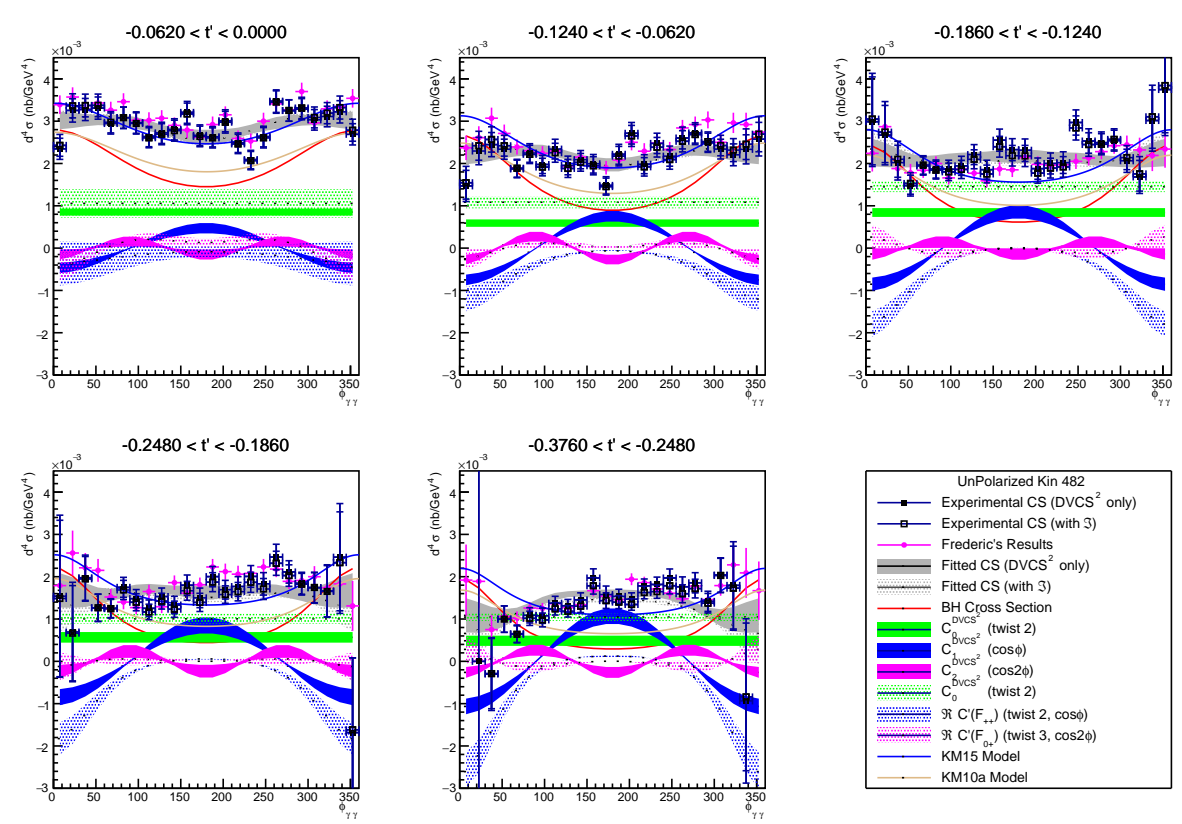

FIG. 67: DVCS unpolarized cross section for Kin 482. Cross section is extracted with 2 different parametrization choices discussed in section 7.2.4. The bands around the fits are the statistical uncertainty. Magenta dot represents a parallel analysis by Frédéric Georges [33]. Model cross sections are given in [57 [56]
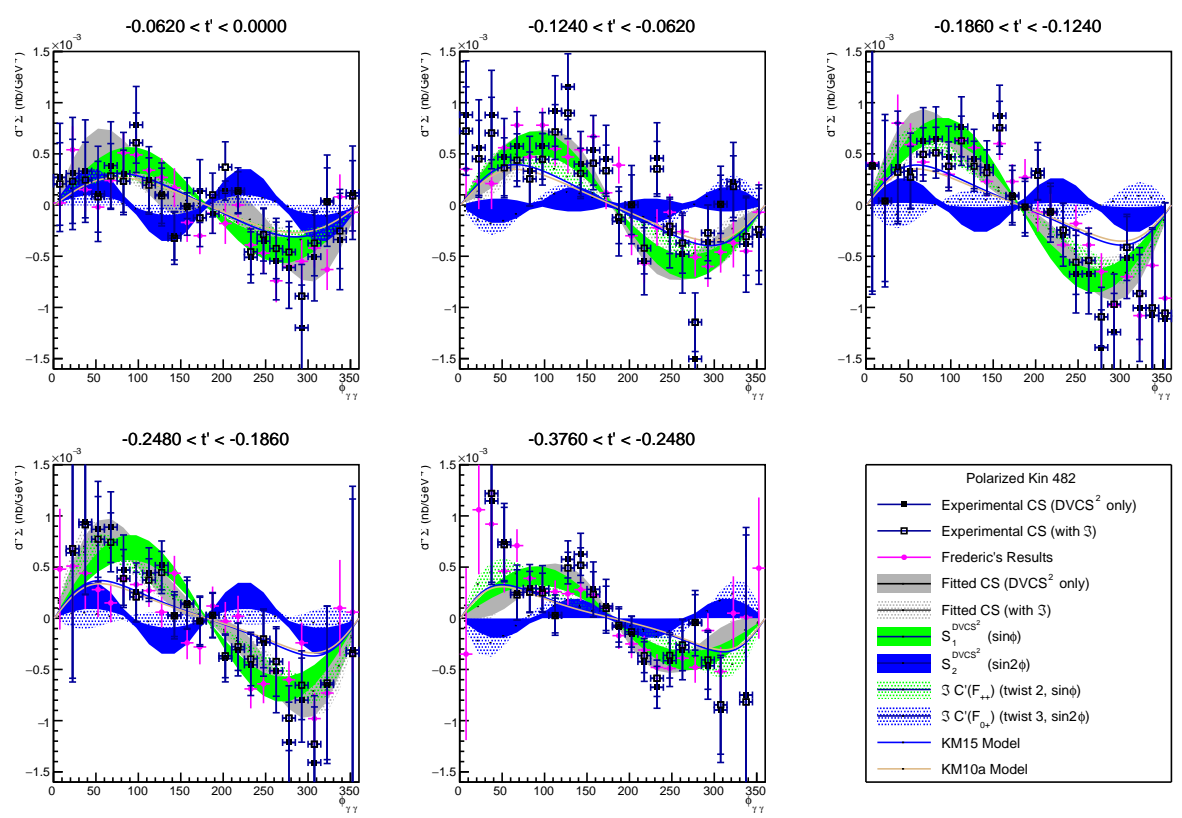

FIG. 68: DVCS helicity dependent cross section for Kin 482. Cross section is extracted with 2 different parametrization choices discussed in section 7.2.4. The bands around the fits are the statistical uncertainty. Magenta dot represents a parallel analysis by Frédéric Georges [33]. Model cross sections are given in [57] [56] 

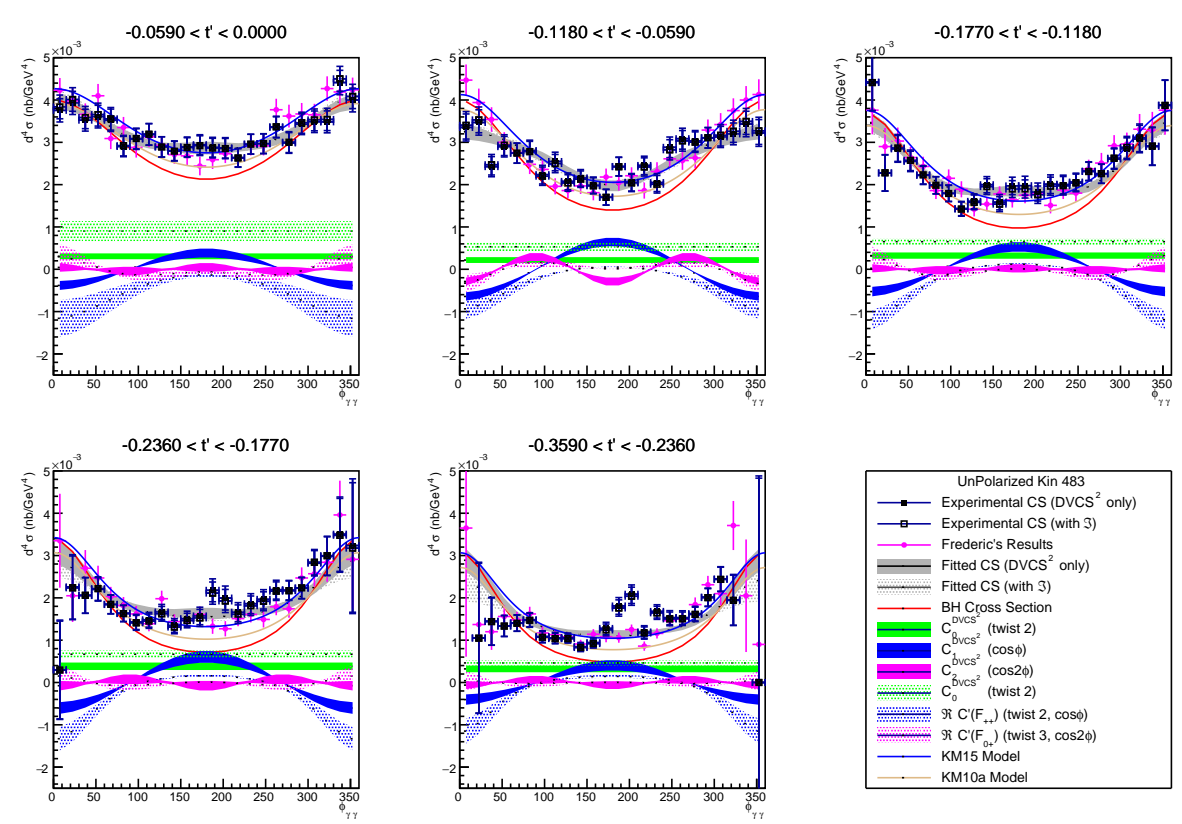

FIG. 69: DVCS unpolarized cross section for Kin 483. Cross section is extracted with 2 different parametrization choices discussed in section 7.2.4. The bands around the fits are the statistical uncertainty. Magenta dot represents a parallel analysis by Frédéric Georges [33]. Model cross sections are given in [57] [56]
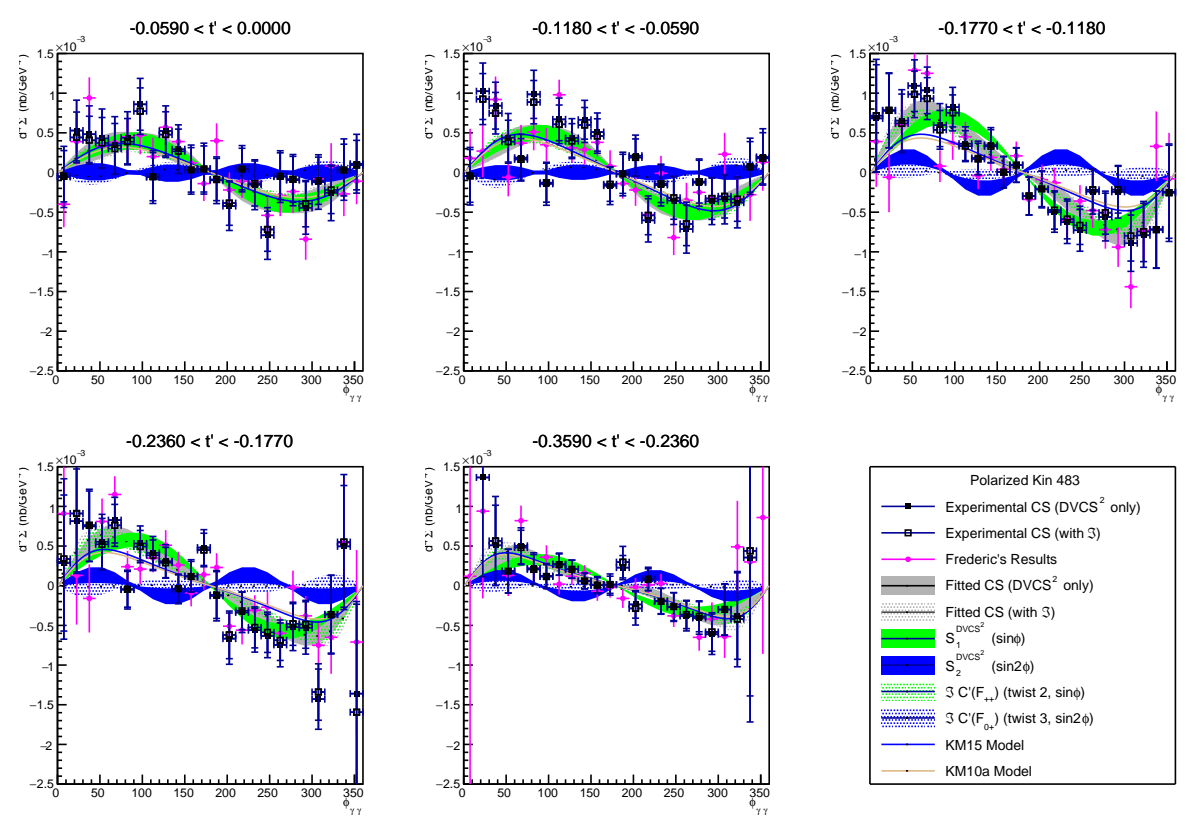

FIG. 70: DVCS helicity dependent cross section for Kin 483. Cross section is extracted with 2 different parametrization choices discussed in section 7.2.4. The bands around the fits are the statistical uncertainty. Magenta dot represents a parallel analysis by Frédéric Georges [33]. Model cross sections are given in [57] [56] 

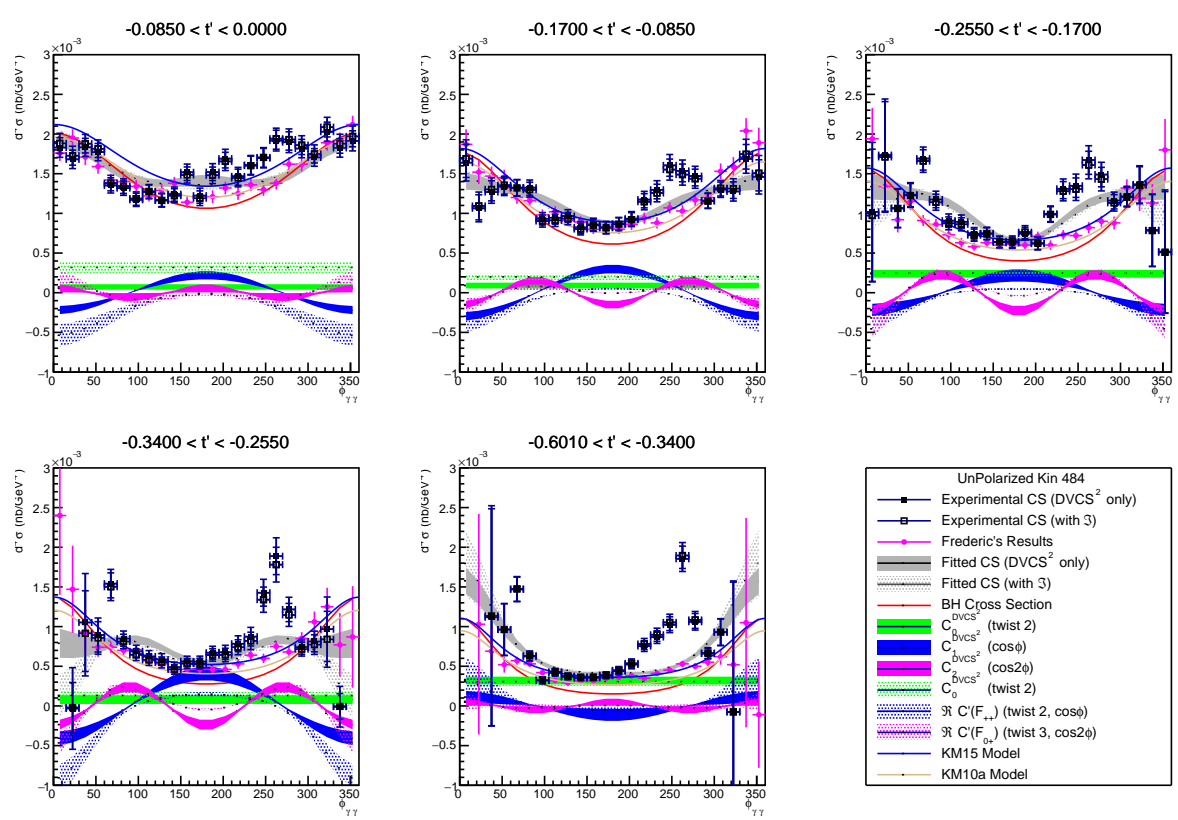

FIG. 71: DVCS unpolarized cross section for Kin 484. Cross section is extracted with 2 different parametrization choices discussed in section 7.2.4. The bands around the fits are the statistical uncertainty. Magenta dot represents a parallel analysis by Frédéric Georges [33]. Model cross sections are given in [57] [56]
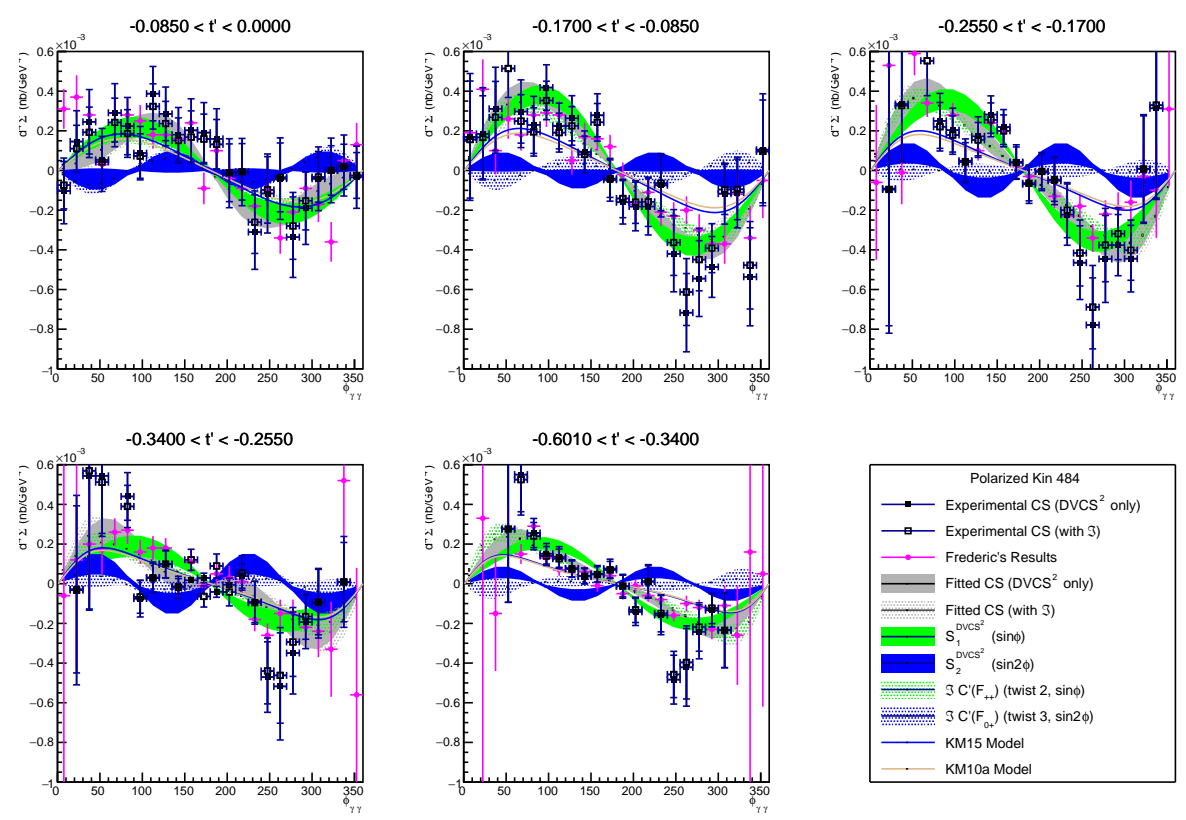

FIG. 72: DVCS helicity dependent cross section for Kin 484. Cross section is extracted with 2 different parametrization choices discussed in section 7.2.4. The bands around the fits are the statistical uncertainty. Magenta dot represents a parallel analysis by Frédéric Georges [33]. Model cross sections are given in [57] [56] 

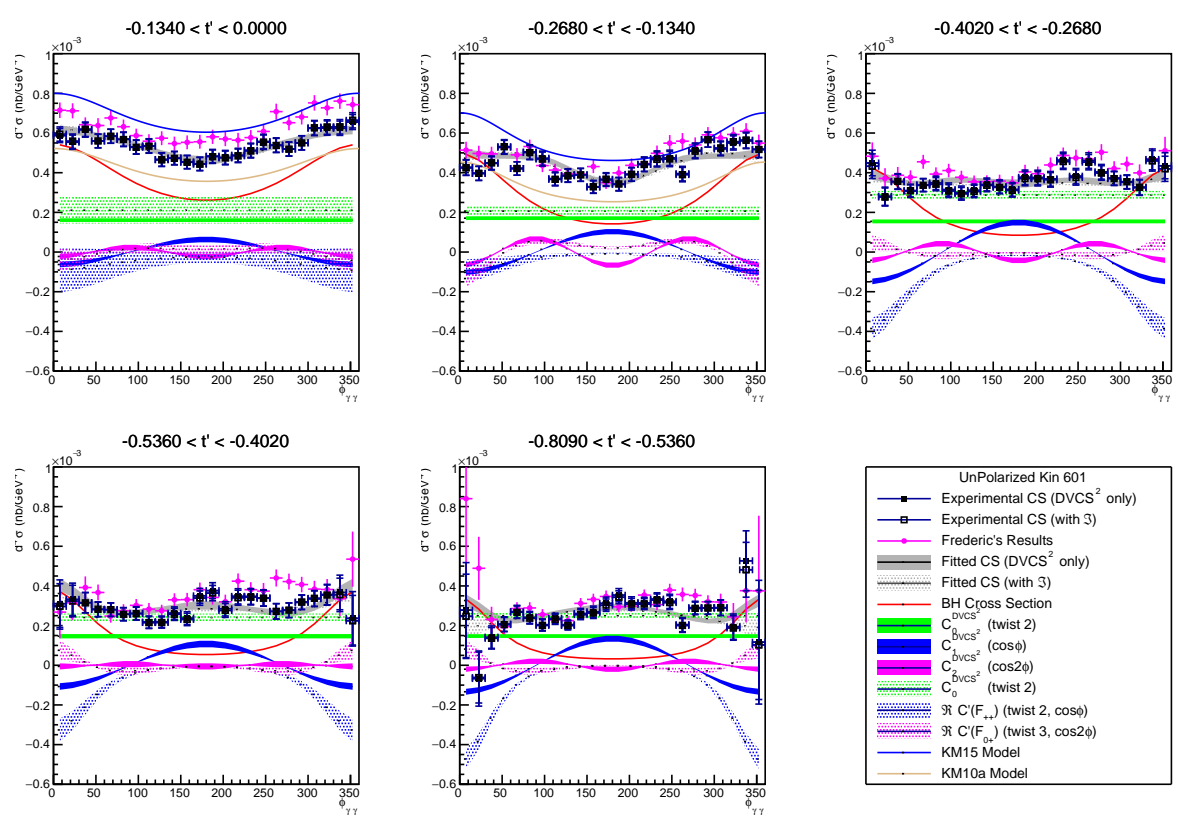

FIG. 73: DVCS unpolarized cross section for Kin 601. Cross section is extracted with 2 different parametrization choices discussed in section 7.2.4. The bands around the fits are the statistical uncertainty. Magenta dot represents a parallel analysis by Frédéric Georges [33]. Model cross sections are given in [57 [56]
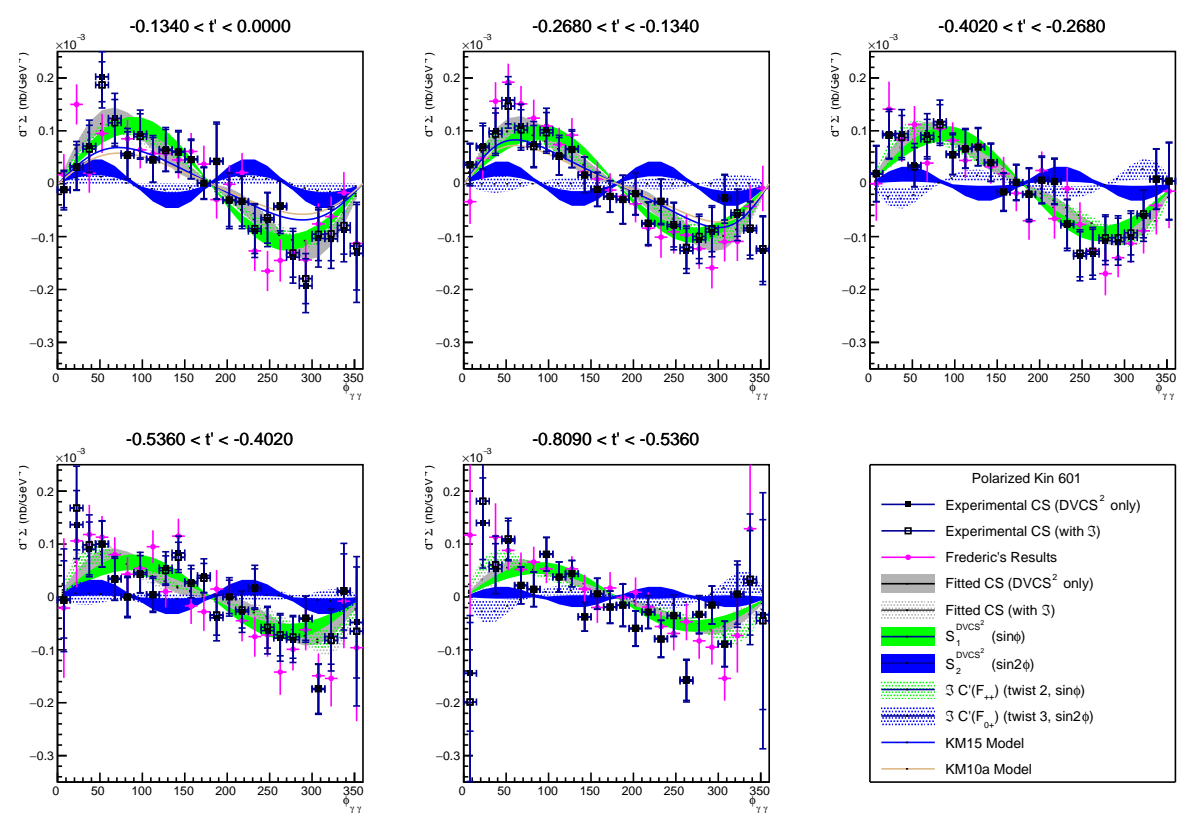

FIG. 74: DVCS helicity dependent cross section for Kin 601. Cross section is extracted with 2 different parametrization choices discussed in section 7.2.4. The bands around the fits are the statistical uncertainty. Magenta dot represents a parallel analysis by Frédéric Georges [33]. Model cross sections are given in [57] [56] 

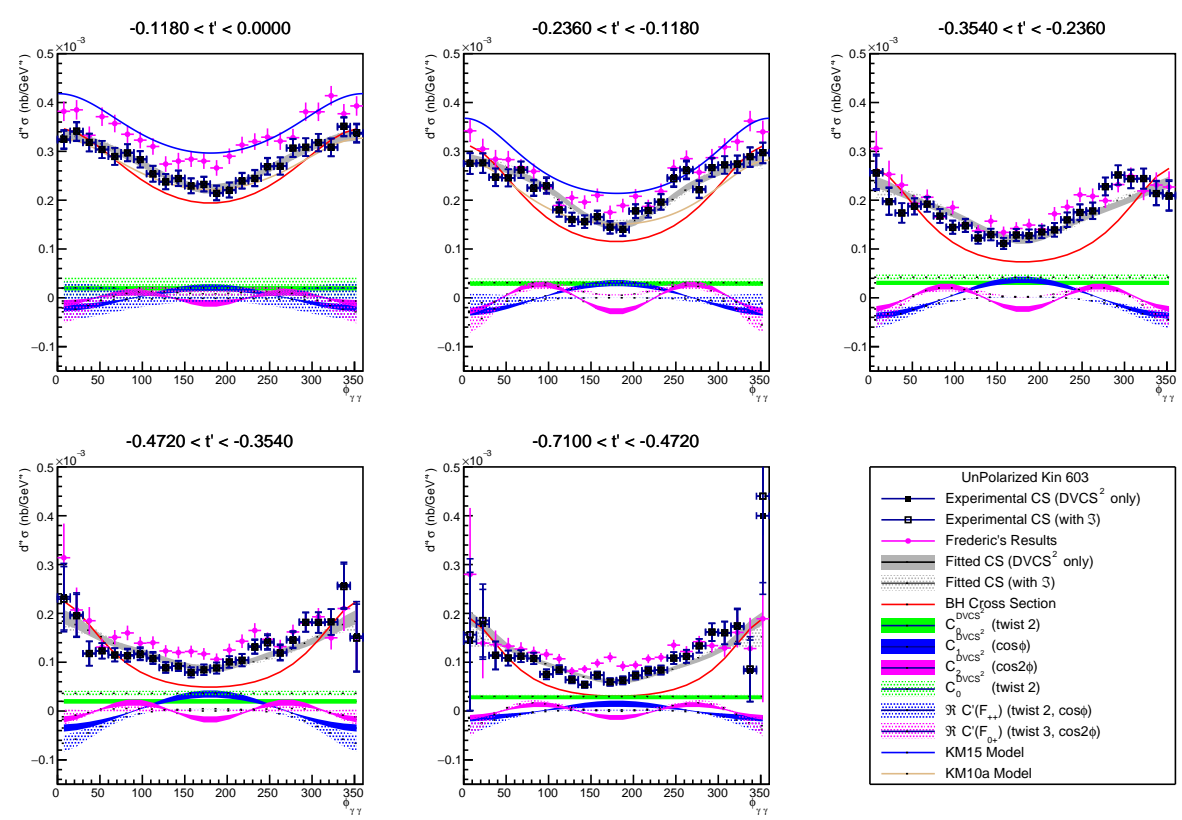

FIG. 75: DVCS unpolarized cross section for Kin 603. Cross section is extracted with 2 different parametrization choices discussed in section 7.2.4. The bands around the fits are the statistical uncertainty. Magenta dot represents a parallel analysis by Frédéric Georges [33]. Model cross sections are given in [57] [56]
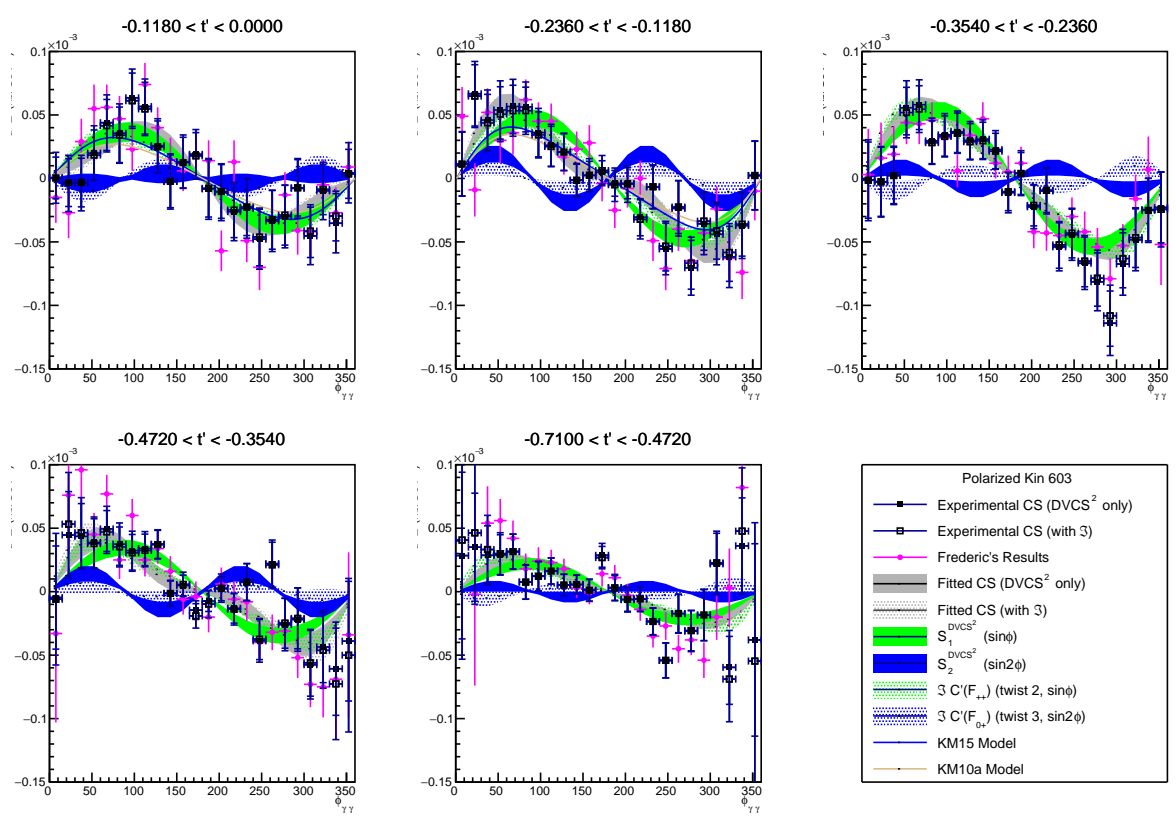

FIG. 76: DVCS helicity dependent cross section for Kin 603. Cross section is extracted with 2 different parametrization choices discussed in section 7.2.4. The bands around the fits are the statistical uncertainty. Magenta dot represents a parallel analysis by Frédéric Georges [33]. Model cross sections are given in [57] [56] 


\section{CHAPTER 8}

\section{CONCLUSIONS}

Generalized Parton Distributions provide co-related transverse spacial and longitudinal momentum distribution of the quarks and gluons making up the nucleon. Deeply Virtual Compton Scattering is the theoretically simple and cleanest process to access GPDs. GPDs appear in DVCS cross section via complex integrals called Compton Form Factors. While the interference between DVCS and virtually indistinguishable competing process Bethe-Heitler amplifies the otherwise smaller DVCS cross section, combined with the high luminosity capabilities of the experimental Hall A at Jefferson Lab, making it possible to measure the cross section, the angular dependencies in the Bethe-Heitler propogators add additional complexities in extracting CFFs. Associated DVCS ep $\rightarrow e \gamma N \pi$ process adds additional layer of complexity in extracting the elastic DVCS events using the missing mass technique.

The DVCS3 experiment was the first experiment to run after the $12 \mathrm{GeV}$ upgrade of JLab and used the first available $10.5 \mathrm{GeV}$ beam. However, this came at a price with new machine and many new unknowns requiring extensive calibration and analysis, including an optics calibration issue caused by the failing Q1 magnet of the Left High Resolution Spectrometer in Spring 2016, the saturation issue of the replaced Q1 magnets at higher current during Fall 2016. During Spring 2016 run period DVCS3 experienced a trigger issue caused by the interference of S0 timing signal with the S2 timing signal causing the trigger to mislabel some $\mathrm{S} 2 \cap$ Cer coincidence triggers as S0กCer coincidences introducing an ambiguity in applying the pre-scale for DIS yield extraction. The trigger issue was studies extensively to minimize this pre-scale ambiguity in DIS analysis and was concluded that the DVCS analysis is immune from it.

HRS detector calibrations were studied and re-calibrations and re-normalizations were performed when needed. A new electron ID cut was developed using re-normalized Pion Rejector energies, effectively eliminating the long non-Poisson tail seen in the Cherenkov spectrum due to multiple low energy electron pile-up and recovering a clean Poisson distribution for the Cherenkov signal. Performance of the Wave Length Shifting Paint on the Cherenkov PMTs were studied and an impressive 50-65\% improvement was found. A long standing multi electron track issue was studied, connecting Pion Rejector, Cherenkov and 
Vertical Drift Chamber to identify the origin, and the nature of the issue and a new correction method was developed to solve the inefficiencies caused by the tracking algorithm when multiple clusters occur in the VDC.

A new photon identification method was developed by analysing up to 9 photons per event to eliminate the systematic uncertainty due to clustering threshold and improving the performances of the $\pi^{0}$ contamination subtraction and accidental coincidence photon subtraction. Systematic uncertainty due to missing mass cut is studied for each kinematic bin in $t^{\prime}$ and $\phi_{\gamma \gamma}$. Preliminary Unpolarized and Helicity Dependent cross sections were extracted in all 9 kinematic point of DVCS3 subdevided in to 120 bins each, with two different choices of parametrization and compared with two models KM15 and KM10a and a parallel analysis. Good agreement between KM15 model and the unpolarized cross section data was found for all the low $x_{B}$ values for which the model is well defined. 


\section{APPENDIX A}

\section{PION REJECTOR RENORMALIZATION PROCEDURE AND STABILITY ANALYSIS}

Following minimization method was developed to renormalize the pion rejector energies as discussed in section 4.1 .

Define a total energy axis on a 2D scatter of $\mathrm{x}$ vs $\mathrm{y}$,

$$
E_{t o t}=x \cos \theta+y \sin \theta
$$

Compute the variance,

$$
\sigma_{t o t}^{2}=\sigma_{x}^{2} \cos ^{2} \theta+\sigma_{y}^{2} \sin ^{2} \theta+\sin 2 \theta(<x y>-<x><y>)
$$

Minimizing the variance with respect to the angle $\theta$ would yeild,

$$
\tan 2 \theta=2 \frac{<x y>-<x><y>}{\sigma_{x}^{2}+\sigma_{y}^{2}}
$$

from this we can extract W1 and W2 as follows,

$$
\begin{aligned}
& W 1=\frac{<E_{t o t}>^{\prime}}{1000 \sin \theta} \\
& W 2=\frac{<E_{t o t}>^{\prime}}{1000 \cos \theta}
\end{aligned}
$$

here,

$$
<E_{t o t}>^{\prime}=<x>\sin \theta+<y>\cos \theta
$$




\section{APPENDIX B}

\section{TABLE OF CROSS SECTIONS}

This section tabulates the extracted cross section in all 9 kinematics. Cross Sections are

given in $p b / G e V^{4}$. $t^{\prime}$ given here is $t-t_{\text {min }}$ and in $G e V^{2} . t_{m i n}$ is defined in equation 126 . All cross section tabulated here are extracted using parametrization choice 2 discussed in section 7.2.4. 


\begin{tabular}{|c|c|c|c|c|c|}
\hline & \multicolumn{5}{|c|}{$\sigma$} \\
\hline$\phi(\operatorname{deg})$ & $\begin{array}{l}\left\langle Q^{2}\right\rangle=3.167 \\
\left\langle x_{B}\right\rangle=0.363 \\
\left\langle t^{\prime}\right\rangle=-0.044\end{array}$ & $\begin{array}{l}\left\langle Q^{2}\right\rangle=3.169 \\
\left\langle x_{B}\right\rangle=0.363 \\
\left\langle t^{\prime}\right\rangle=-0.130\end{array}$ & $\begin{array}{l}\left\langle Q^{2}\right\rangle=3.180 \\
\left\langle x_{B}\right\rangle=0.365 \\
\left\langle t^{\prime}\right\rangle=-0.216\end{array}$ & $\begin{array}{l}\left\langle Q^{2}\right\rangle=3.184 \\
\left\langle x_{B}\right\rangle=0.365 \\
\left\langle t^{\prime}\right\rangle=-0.302\end{array}$ & $\begin{array}{l}\left\langle Q^{2}\right\rangle=3.180 \\
\left\langle x_{B}\right\rangle=0.364 \\
\left\langle t^{\prime}\right\rangle=-0.418\end{array}$ \\
\hline 7.5 & $45.37 \pm 1.44$ & $42.27 \pm 1.69$ & $33.20 \pm 2.52$ & $24.04 \pm 6.63$ & $8.12 \pm 28.77$ \\
\hline 22.5 & $42.59 \pm 1.39$ & $36.56 \pm 1.51$ & $28.31 \pm 1.98$ & $18.40 \pm 3.75$ & $13.64 \pm 9.43$ \\
\hline 37.5 & $38.79 \pm 1.31$ & $36.61 \pm 1.40$ & $22.69 \pm 1.38$ & $15.50 \pm 1.72$ & $10.36 \pm 2.07$ \\
\hline 52.5 & $35.25 \pm 1.24$ & $27.81 \pm 1.20$ & $17.61 \pm 1.07$ & $15.02 \pm 1.17$ & $9.83 \pm 1.07$ \\
\hline 67.5 & $32.53 \pm 1.16$ & $22.41 \pm 1.01$ & $16.34 \pm 0.91$ & $10.94 \pm 0.92$ & $7.58 \pm 0.78$ \\
\hline 82.5 & $27.91 \pm 1.07$ & $18.99 \pm 0.93$ & $12.64 \pm 0.79$ & $9.55 \pm 0.80$ & $6.91 \pm 0.69$ \\
\hline 97.5 & $25.52 \pm 1.00$ & $16.07 \pm 0.84$ & $10.16 \pm 0.68$ & $8.85 \pm 0.72$ & $5.51 \pm 0.58$ \\
\hline 112.5 & $21.11 \pm 0.92$ & $14.26 \pm 0.78$ & $10.19 \pm 0.66$ & $6.84 \pm 0.63$ & $4.93 \pm 0.47$ \\
\hline 127.5 & $22.31 \pm 0.93$ & $13.48 \pm 0.75$ & $9.15 \pm 0.65$ & $6.48 \pm 0.57$ & $4.15 \pm 0.42$ \\
\hline 142.5 & $19.60 \pm 0.88$ & $13.18 \pm 0.75$ & $7.43 \pm 0.61$ & $5.54 \pm 0.55$ & $5.19 \pm 0.49$ \\
\hline 157.5 & $20.18 \pm 0.88$ & $11.65 \pm 0.72$ & $8.95 \pm 0.66$ & $6.49 \pm 0.61$ & $4.75 \pm 0.63$ \\
\hline 172.5 & $18.98 \pm 0.86$ & $11.26 \pm 0.71$ & $9.82 \pm 0.71$ & $6.27 \pm 0.68$ & $5.87 \pm 0.89$ \\
\hline 187.5 & $20.63 \pm 0.90$ & $11.89 \pm 0.74$ & $9.07 \pm 0.68$ & $6.03 \pm 0.65$ & $6.74 \pm 0.92$ \\
\hline 202.5 & $20.52 \pm 0.90$ & $13.69 \pm 0.76$ & $10.40 \pm 0.73$ & $8.25 \pm 0.72$ & $7.38 \pm 0.84$ \\
\hline 217.5 & $19.75 \pm 0.89$ & $14.59 \pm 0.80$ & $11.15 \pm 0.78$ & $8.49 \pm 0.78$ & $8.96 \pm 0.79$ \\
\hline 232.5 & $21.11 \pm 0.93$ & $13.73 \pm 0.79$ & $8.98 \pm 0.69$ & $8.82 \pm 0.77$ & $10.93 \pm 0.79$ \\
\hline 247.5 & $23.99 \pm 0.99$ & $16.16 \pm 0.87$ & $11.17 \pm 0.73$ & $7.87 \pm 0.67$ & $6.50 \pm 0.58$ \\
\hline 262.5 & $25.56 \pm 1.02$ & $19.54 \pm 0.95$ & $12.11 \pm 0.78$ & $10.21 \pm 0.78$ & $6.86 \pm 0.64$ \\
\hline 277.5 & $27.02 \pm 1.06$ & $22.99 \pm 1.03$ & $14.48 \pm 0.90$ & $13.12 \pm 0.94$ & $7.69 \pm 0.79$ \\
\hline 292.5 & $32.83 \pm 1.18$ & $27.63 \pm 1.20$ & $18.74 \pm 1.08$ & $15.00 \pm 1.15$ & $12.82 \pm 1.02$ \\
\hline 307.5 & $35.71 \pm 1.23$ & $32.16 \pm 1.34$ & $25.82 \pm 1.34$ & $21.37 \pm 1.51$ & $20.23 \pm 1.64$ \\
\hline 322.5 & $42.35 \pm 1.36$ & $40.58 \pm 1.49$ & $30.80 \pm 1.57$ & $25.26 \pm 2.00$ & $22.25 \pm 3.03$ \\
\hline 337.5 & $45.69 \pm 1.45$ & $48.14 \pm 1.69$ & $39.09 \pm 2.12$ & $29.16 \pm 4.02$ & $17.34 \pm 10.67$ \\
\hline 352.5 & $46.04 \pm 1.46$ & $46.32 \pm 1.74$ & $36.92 \pm 2.71$ & $20.60 \pm 6.64$ & $-16.43 \pm 51.57$ \\
\hline
\end{tabular}

TABLE 20: Unpolarized cross section for Kin 361 in $\mathrm{pb} / \mathrm{GeV}^{4}$ 


\begin{tabular}{|c|c|c|c|c|c|}
\hline & \multicolumn{5}{|c|}{$\sigma$} \\
\hline$\phi(\operatorname{deg})$ & $\begin{array}{l}\left\langle Q^{2}\right\rangle=3.167 \\
\left\langle x_{B}\right\rangle=0.363 \\
\left\langle t^{\prime}\right\rangle=-0.044\end{array}$ & $\begin{array}{l}\left\langle Q^{2}\right\rangle=3.169 \\
\left\langle x_{B}\right\rangle=0.363 \\
\left\langle t^{\prime}\right\rangle=-0.130\end{array}$ & $\begin{array}{l}\left\langle Q^{2}\right\rangle=3.180 \\
\left\langle x_{B}\right\rangle=0.365 \\
\left\langle t^{\prime}\right\rangle=-0.216\end{array}$ & $\begin{array}{l}\left\langle Q^{2}\right\rangle=3.184 \\
\left\langle x_{B}\right\rangle=0.365 \\
\left\langle t^{\prime}\right\rangle=-0.302\end{array}$ & $\begin{array}{l}\left\langle Q^{2}\right\rangle=3.180 \\
\left\langle x_{B}\right\rangle=0.364 \\
\left\langle t^{\prime}\right\rangle=-0.418\end{array}$ \\
\hline 7.5 & $1.75 \pm 1.78$ & $-0.81 \pm 1.97$ & $3.13 \pm 2.80$ & $-2.13 \pm 5.36$ & $-30.70 \pm 22.53$ \\
\hline 22.5 & $3.99 \pm 1.65$ & $0.07 \pm 1.74$ & $1.72 \pm 2.24$ & $2.47 \pm 3.31$ & $15.66 \pm 8.88$ \\
\hline 37.5 & $2.92 \pm 1.56$ & $6.57 \pm 1.61$ & $5.02 \pm 1.61$ & $2.77 \pm 1.70$ & $4.51 \pm 2.12$ \\
\hline 52.5 & $5.50 \pm 1.50$ & $6.32 \pm 1.36$ & $5.66 \pm 1.26$ & $4.13 \pm 1.26$ & $2.01 \pm 1.19$ \\
\hline 67.5 & $4.36 \pm 1.43$ & $7.31 \pm 1.15$ & $3.43 \pm 1.05$ & $1.31 \pm 1.01$ & $2.93 \pm 0.87$ \\
\hline 82.5 & $5.32 \pm 1.32$ & $6.05 \pm 1.05$ & $3.82 \pm 0.89$ & $2.29 \pm 0.90$ & $1.29 \pm 0.76$ \\
\hline 97.5 & $6.98 \pm 1.25$ & $2.91 \pm 0.95$ & $2.62 \pm 0.76$ & $2.74 \pm 0.82$ & $0.49 \pm 0.63$ \\
\hline 112.5 & $4.47 \pm 1.15$ & $4.43 \pm 0.86$ & $2.36 \pm 0.72$ & $2.62 \pm 0.72$ & $0.52 \pm 0.50$ \\
\hline 127.5 & $4.75 \pm 1.16$ & $3.24 \pm 0.82$ & $2.50 \pm 0.69$ & $0.98 \pm 0.67$ & $0.65 \pm 0.43$ \\
\hline 142.5 & $2.28 \pm 1.10$ & $0.99 \pm 0.81$ & $0.92 \pm 0.63$ & $0.80 \pm 0.66$ & $0.70 \pm 0.46$ \\
\hline 157.5 & $3.13 \pm 1.12$ & $1.90 \pm 0.78$ & $1.39 \pm 0.66$ & $1.57 \pm 0.74$ & $0.15 \pm 0.55$ \\
\hline 172.5 & $1.62 \pm 1.17$ & $-1.01 \pm 0.78$ & $-0.30 \pm 0.72$ & $0.83 \pm 0.87$ & $1.17 \pm 0.72$ \\
\hline 187.5 & $-0.43 \pm 1.02$ & $0.40 \pm 0.75$ & $0.01 \pm 0.65$ & $-0.45 \pm 0.78$ & $-0.60 \pm 0.73$ \\
\hline 202.5 & $-0.52 \pm 1.08$ & $-0.72 \pm 0.81$ & $-0.19 \pm 0.73$ & $-0.72 \pm 0.88$ & $-1.29 \pm 0.73$ \\
\hline 217.5 & $-2.69 \pm 1.08$ & $-2.49 \pm 0.87$ & $-0.66 \pm 0.80$ & $-1.91 \pm 0.93$ & $-1.20 \pm 0.75$ \\
\hline 232.5 & $-4.52 \pm 1.14$ & $-1.32 \pm 0.86$ & $-1.47 \pm 0.73$ & $-2.07 \pm 0.88$ & $-0.57 \pm 0.80$ \\
\hline 247.5 & $-5.31 \pm 1.21$ & $-4.76 \pm 0.97$ & $-2.00 \pm 0.80$ & $-3.05 \pm 0.76$ & $-2.05 \pm 0.61$ \\
\hline 262.5 & $-6.36 \pm 1.25$ & $-4.33 \pm 1.07$ & $-2.35 \pm 0.86$ & $-1.61 \pm 0.88$ & $-2.60 \pm 0.70$ \\
\hline 277.5 & $-4.17 \pm 1.29$ & $-4.67 \pm 1.16$ & $-4.46 \pm 1.02$ & $-4.43 \pm 1.05$ & $-2.00 \pm 0.87$ \\
\hline 292.5 & $-5.35 \pm 1.41$ & $-6.93 \pm 1.35$ & $-8.17 \pm 1.25$ & $-4.35 \pm 1.27$ & $-3.49 \pm 1.13$ \\
\hline 307.5 & $-6.75 \pm 1.44$ & $-7.68 \pm 1.50$ & $-7.72 \pm 1.58$ & $-4.40 \pm 1.64$ & $-6.32 \pm 1.80$ \\
\hline 322.5 & $-2.09 \pm 1.55$ & $-6.19 \pm 1.67$ & $-7.79 \pm 1.84$ & $-2.55 \pm 2.00$ & $-2.24 \pm 3.12$ \\
\hline 337.5 & $-2.58 \pm 1.60$ & $-4.56 \pm 1.87$ & $-5.57 \pm 2.36$ & $-6.77 \pm 3.49$ & $-8.19 \pm 9.97$ \\
\hline 352.5 & $-3.88 \pm 1.48$ & $1.00 \pm 1.86$ & $-3.91 \pm 2.86$ & $7.33 \pm 5.12$ & $-20.43 \pm 35.97$ \\
\hline
\end{tabular}

TABLE 21: Helicity-Dependent cross section for Kin 361 in pb/GeV 


\begin{tabular}{|c|c|c|c|c|c|}
\hline & \multicolumn{5}{|c|}{$\sigma$} \\
\hline$\phi(\operatorname{deg})$ & $\begin{array}{l}\left\langle Q^{2}\right\rangle=3.650 \\
\left\langle x_{B}\right\rangle=0.367 \\
\left\langle t^{\prime}\right\rangle=-0.032\end{array}$ & $\begin{array}{l}\left\langle Q^{2}\right\rangle=3.653 \\
\left\langle x_{B}\right\rangle=0.367 \\
\left\langle t^{\prime}\right\rangle=-0.093\end{array}$ & $\begin{array}{l}\left\langle Q^{2}\right\rangle=3.669 \\
\left\langle x_{B}\right\rangle=0.369 \\
\left\langle t^{\prime}\right\rangle=-0.155\end{array}$ & $\begin{array}{l}\left\langle Q^{2}\right\rangle=3.678 \\
\left\langle x_{B}\right\rangle=0.370 \\
\left\langle t^{\prime}\right\rangle=-0.216\end{array}$ & $\begin{array}{l}\left\langle Q^{2}\right\rangle=3.679 \\
\left\langle x_{B}\right\rangle=0.370 \\
\left\langle t^{\prime}\right\rangle=-0.304\end{array}$ \\
\hline 7.5 & $25.73 \pm 0.88$ & $23.85 \pm 1.02$ & $20.82 \pm 1.48$ & $21.21 \pm 2.80$ & $13.05 \pm 5.98$ \\
\hline 22.5 & $23.94 \pm 0.85$ & $23.79 \pm 0.98$ & $17.39 \pm 1.17$ & $15.14 \pm 1.91$ & $12.01 \pm 3.05$ \\
\hline 37.5 & $22.66 \pm 0.83$ & $20.16 \pm 0.86$ & $17.08 \pm 0.96$ & $11.34 \pm 1.08$ & $8.79 \pm 1.26$ \\
\hline 52.5 & $20.22 \pm 0.78$ & $19.12 \pm 0.81$ & $15.61 \pm 0.78$ & $11.44 \pm 0.80$ & $9.38 \pm 0.70$ \\
\hline 67.5 & $20.06 \pm 0.76$ & $17.43 \pm 0.76$ & $13.97 \pm 0.72$ & $10.42 \pm 0.67$ & $8.51 \pm 0.56$ \\
\hline 82.5 & $18.72 \pm 0.73$ & $14.70 \pm 0.68$ & $10.91 \pm 0.62$ & $8.87 \pm 0.59$ & $6.39 \pm 0.45$ \\
\hline 97.5 & $16.57 \pm 0.70$ & $12.88 \pm 0.64$ & $8.53 \pm 0.55$ & $7.18 \pm 0.52$ & $5.71 \pm 0.39$ \\
\hline 112.5 & $16.07 \pm 0.67$ & $11.05 \pm 0.58$ & $8.03 \pm 0.50$ & $6.40 \pm 0.46$ & $4.74 \pm 0.32$ \\
\hline 127.5 & $14.98 \pm 0.63$ & $10.23 \pm 0.55$ & $8.07 \pm 0.49$ & $6.23 \pm 0.43$ & $4.10 \pm 0.27$ \\
\hline 142.5 & $13.06 \pm 0.59$ & $9.78 \pm 0.52$ & $7.12 \pm 0.46$ & $4.96 \pm 0.40$ & $4.11 \pm 0.27$ \\
\hline 157.5 & $13.74 \pm 0.61$ & $8.19 \pm 0.48$ & $6.72 \pm 0.45$ & $5.44 \pm 0.41$ & $4.52 \pm 0.29$ \\
\hline 172.5 & $11.97 \pm 0.57$ & $8.93 \pm 0.50$ & $7.18 \pm 0.46$ & $4.98 \pm 0.41$ & $3.57 \pm 0.28$ \\
\hline 187.5 & $12.29 \pm 0.58$ & $8.19 \pm 0.47$ & $6.88 \pm 0.46$ & $4.81 \pm 0.40$ & $4.36 \pm 0.29$ \\
\hline 202.5 & $12.75 \pm 0.60$ & $9.20 \pm 0.50$ & $6.76 \pm 0.45$ & $5.39 \pm 0.40$ & $3.92 \pm 0.26$ \\
\hline 217.5 & $13.84 \pm 0.62$ & $10.19 \pm 0.55$ & $6.58 \pm 0.45$ & $5.07 \pm 0.40$ & $3.89 \pm 0.26$ \\
\hline 232.5 & $13.31 \pm 0.62$ & $10.52 \pm 0.56$ & $7.35 \pm 0.48$ & $6.09 \pm 0.42$ & $4.21 \pm 0.27$ \\
\hline 247.5 & $16.26 \pm 0.68$ & $11.62 \pm 0.59$ & $9.39 \pm 0.53$ & $5.80 \pm 0.45$ & $4.90 \pm 0.32$ \\
\hline 262.5 & $18.02 \pm 0.72$ & $12.65 \pm 0.61$ & $9.48 \pm 0.55$ & $7.21 \pm 0.51$ & $5.97 \pm 0.36$ \\
\hline 277.5 & $20.62 \pm 0.77$ & $14.49 \pm 0.68$ & $11.51 \pm 0.61$ & $8.42 \pm 0.55$ & $6.46 \pm 0.41$ \\
\hline 292.5 & $20.71 \pm 0.78$ & $18.82 \pm 0.76$ & $14.92 \pm 0.71$ & $11.20 \pm 0.68$ & $8.89 \pm 0.53$ \\
\hline 307.5 & $21.46 \pm 0.80$ & $19.21 \pm 0.77$ & $17.39 \pm 0.77$ & $11.38 \pm 0.75$ & $10.43 \pm 0.72$ \\
\hline 322.5 & $24.01 \pm 0.84$ & $23.33 \pm 0.86$ & $17.62 \pm 0.90$ & $14.46 \pm 1.10$ & $12.21 \pm 1.25$ \\
\hline 337.5 & $22.68 \pm 0.83$ & $23.87 \pm 0.93$ & $19.37 \pm 1.19$ & $22.40 \pm 1.90$ & $13.70 \pm 2.89$ \\
\hline 352.5 & $25.80 \pm 0.89$ & $23.64 \pm 0.99$ & $22.05 \pm 1.48$ & $16.39 \pm 2.76$ & $21.75 \pm 8.31$ \\
\hline
\end{tabular}

TABLE 22: Unpolarized cross section for Kin 362 in $\mathrm{pb} / \mathrm{GeV}^{4}$ 


\begin{tabular}{|c|c|c|c|c|c|}
\hline & \multicolumn{5}{|c|}{$\sigma$} \\
\hline$\phi(\operatorname{deg})$ & $\begin{array}{l}\left\langle Q^{2}\right\rangle=3.650 \\
\left\langle x_{B}\right\rangle=0.367 \\
\left\langle t^{\prime}\right\rangle=-0.032\end{array}$ & $\begin{array}{l}\left\langle Q^{2}\right\rangle=3.653 \\
\left\langle x_{B}\right\rangle=0.367 \\
\left\langle t^{\prime}\right\rangle=-0.093\end{array}$ & $\begin{array}{l}\left\langle Q^{2}\right\rangle=3.669 \\
\left\langle x_{B}\right\rangle=0.369 \\
\left\langle t^{\prime}\right\rangle=-0.155\end{array}$ & $\begin{array}{l}\left\langle Q^{2}\right\rangle=3.678 \\
\left\langle x_{B}\right\rangle=0.370 \\
\left\langle t^{\prime}\right\rangle=-0.216\end{array}$ & $\begin{array}{l}\left\langle Q^{2}\right\rangle=3.679 \\
\left\langle x_{B}\right\rangle=0.370 \\
\left\langle t^{\prime}\right\rangle=-0.304\end{array}$ \\
\hline 7.5 & $2.01 \pm 0.75$ & $1.03 \pm 0.96$ & $3.08 \pm 1.43$ & $-0.39 \pm 2.26$ & $-2.68 \pm 4.50$ \\
\hline 22.5 & $1.62 \pm 0.88$ & $2.82 \pm 1.03$ & $4.60 \pm 1.27$ & $0.90 \pm 1.75$ & $5.18 \pm 2.62$ \\
\hline 37.5 & $1.39 \pm 0.90$ & $2.68 \pm 0.94$ & $4.10 \pm 1.10$ & $2.47 \pm 1.11$ & $2.51 \pm 1.25$ \\
\hline 52.5 & $1.98 \pm 0.88$ & $3.45 \pm 0.90$ & $4.77 \pm 0.91$ & $3.01 \pm 0.89$ & $1.48 \pm 0.76$ \\
\hline 67.5 & $4.84 \pm 0.88$ & $5.65 \pm 0.86$ & $4.03 \pm 0.84$ & $3.73 \pm 0.76$ & $2.49 \pm 0.62$ \\
\hline 82.5 & $3.41 \pm 0.86$ & $4.20 \pm 0.78$ & $2.85 \pm 0.70$ & $2.18 \pm 0.68$ & $2.38 \pm 0.50$ \\
\hline 97.5 & $3.18 \pm 0.84$ & $4.28 \pm 0.73$ & $3.96 \pm 0.62$ & $2.00 \pm 0.59$ & $1.51 \pm 0.44$ \\
\hline 112.5 & $4.32 \pm 0.81$ & $3.13 \pm 0.65$ & $1.28 \pm 0.54$ & $2.09 \pm 0.52$ & $1.46 \pm 0.35$ \\
\hline 127.5 & $3.37 \pm 0.76$ & $2.43 \pm 0.62$ & $1.30 \pm 0.52$ & $1.94 \pm 0.49$ & $0.75 \pm 0.29$ \\
\hline 142.5 & $1.70 \pm 0.70$ & $1.12 \pm 0.57$ & $1.21 \pm 0.46$ & $1.17 \pm 0.46$ & $0.80 \pm 0.29$ \\
\hline 157.5 & $1.05 \pm 0.69$ & $0.80 \pm 0.51$ & $0.90 \pm 0.43$ & $0.08 \pm 0.47$ & $0.17 \pm 0.31$ \\
\hline 172.5 & $1.22 \pm 0.54$ & $0.81 \pm 0.47$ & $0.43 \pm 0.39$ & $0.84 \pm 0.44$ & $0.63 \pm 0.28$ \\
\hline 187.5 & $-1.94 \pm 1.06$ & $-0.88 \pm 0.62$ & $-0.06 \pm 0.50$ & $-0.06 \pm 0.53$ & $-0.17 \pm 0.34$ \\
\hline 202.5 & $-2.39 \pm 0.83$ & $-0.35 \pm 0.58$ & $-0.12 \pm 0.46$ & $-0.74 \pm 0.49$ & $-0.48 \pm 0.28$ \\
\hline 217.5 & $-2.18 \pm 0.80$ & $-2.09 \pm 0.62$ & $-0.89 \pm 0.47$ & $-1.04 \pm 0.49$ & $-0.74 \pm 0.28$ \\
\hline 232.5 & $-1.17 \pm 0.80$ & $-2.68 \pm 0.64$ & $-1.81 \pm 0.50$ & $-1.13 \pm 0.50$ & $-0.80 \pm 0.30$ \\
\hline 247.5 & $-3.47 \pm 0.86$ & $-3.83 \pm 0.68$ & $-1.35 \pm 0.57$ & $-1.66 \pm 0.52$ & $-1.84 \pm 0.35$ \\
\hline 262.5 & $-3.69 \pm 0.91$ & $-3.46 \pm 0.71$ & $-2.40 \pm 0.61$ & $-2.17 \pm 0.59$ & $-1.84 \pm 0.40$ \\
\hline 277.5 & $-4.23 \pm 0.97$ & $-2.74 \pm 0.79$ & $-2.75 \pm 0.69$ & $-3.18 \pm 0.63$ & $-2.36 \pm 0.46$ \\
\hline 292.5 & $-3.19 \pm 0.97$ & $-4.65 \pm 0.89$ & $-5.44 \pm 0.83$ & $-2.91 \pm 0.78$ & $-2.18 \pm 0.59$ \\
\hline 307.5 & $-3.05 \pm 0.98$ & $-3.57 \pm 0.91$ & $-3.78 \pm 0.92$ & $-2.03 \pm 0.85$ & $-2.27 \pm 0.80$ \\
\hline 322.5 & $-2.26 \pm 1.03$ & $-4.55 \pm 1.01$ & $-4.23 \pm 1.08$ & $-2.58 \pm 1.15$ & $-3.01 \pm 1.28$ \\
\hline 337.5 & $0.62 \pm 1.07$ & $-0.58 \pm 1.10$ & $-1.26 \pm 1.39$ & $-2.59 \pm 1.82$ & $0.08 \pm 2.65$ \\
\hline 352.5 & $-0.75 \pm 1.44$ & $-4.20 \pm 1.29$ & $2.32 \pm 1.80$ & $-2.74 \pm 2.64$ & $-5.82 \pm 7.57$ \\
\hline
\end{tabular}

TABLE 23: Helicity-Dependent cross section for Kin 362 in pb/GeV 


\begin{tabular}{|c|c|c|c|c|c|}
\hline & \multicolumn{5}{|c|}{$\sigma$} \\
\hline$\phi(\operatorname{deg})$ & $\begin{array}{l}\left\langle Q^{2}\right\rangle=4.532 \\
\left\langle x_{B}\right\rangle=0.369 \\
\left\langle t^{\prime}\right\rangle=-0.031\end{array}$ & $\begin{array}{l}\left\langle Q^{2}\right\rangle=4.550 \\
\left\langle x_{B}\right\rangle=0.370 \\
\left\langle t^{\prime}\right\rangle=-0.093\end{array}$ & $\begin{array}{l}\left\langle Q^{2}\right\rangle=4.574 \\
\left\langle x_{B}\right\rangle=0.372 \\
\left\langle t^{\prime}\right\rangle=-0.154\end{array}$ & $\begin{array}{l}\left\langle Q^{2}\right\rangle=4.585 \\
\left\langle x_{B}\right\rangle=0.373 \\
\left\langle t^{\prime}\right\rangle=-0.216\end{array}$ & $\begin{array}{l}\left\langle Q^{2}\right\rangle=4.568 \\
\left\langle x_{B}\right\rangle=0.371 \\
\left\langle t^{\prime}\right\rangle=-0.303\end{array}$ \\
\hline 7.5 & $11.97 \pm 0.56$ & $12.81 \pm 0.73$ & $8.11 \pm 0.96$ & $9.22 \pm 1.67$ & $9.05 \pm 3.34$ \\
\hline 22.5 & $11.69 \pm 0.54$ & $10.82 \pm 0.62$ & $9.55 \pm 0.85$ & $7.51 \pm 1.25$ & $5.06 \pm 1.66$ \\
\hline 37.5 & $11.34 \pm 0.55$ & $9.28 \pm 0.54$ & $8.04 \pm 0.63$ & $6.35 \pm 0.76$ & $4.72 \pm 0.81$ \\
\hline 52.5 & $10.83 \pm 0.51$ & $8.74 \pm 0.49$ & $6.98 \pm 0.49$ & $6.57 \pm 0.57$ & $4.32 \pm 0.49$ \\
\hline 67.5 & $10.43 \pm 0.50$ & $8.09 \pm 0.46$ & $6.74 \pm 0.45$ & $5.07 \pm 0.45$ & $3.81 \pm 0.37$ \\
\hline 82.5 & $9.51 \pm 0.48$ & $7.05 \pm 0.42$ & $6.00 \pm 0.40$ & $4.85 \pm 0.42$ & $3.24 \pm 0.32$ \\
\hline 97.5 & $8.62 \pm 0.46$ & $6.77 \pm 0.42$ & $5.08 \pm 0.38$ & $4.57 \pm 0.40$ & $3.37 \pm 0.33$ \\
\hline 112.5 & $8.40 \pm 0.45$ & $6.58 \pm 0.42$ & $5.06 \pm 0.39$ & $4.54 \pm 0.41$ & $3.64 \pm 0.32$ \\
\hline 127.5 & $8.14 \pm 0.45$ & $7.13 \pm 0.44$ & $4.39 \pm 0.37$ & $4.54 \pm 0.40$ & $2.95 \pm 0.28$ \\
\hline 142.5 & $7.79 \pm 0.43$ & $6.33 \pm 0.42$ & $5.34 \pm 0.40$ & $4.10 \pm 0.38$ & $3.15 \pm 0.25$ \\
\hline 157.5 & $7.20 \pm 0.42$ & $5.99 \pm 0.40$ & $4.53 \pm 0.37$ & $3.86 \pm 0.38$ & $2.61 \pm 0.25$ \\
\hline 172.5 & $7.13 \pm 0.41$ & $5.85 \pm 0.39$ & $4.39 \pm 0.37$ & $3.71 \pm 0.37$ & $2.59 \pm 0.26$ \\
\hline 187.5 & $7.11 \pm 0.42$ & $5.28 \pm 0.38$ & $3.76 \pm 0.34$ & $4.04 \pm 0.39$ & $3.26 \pm 0.31$ \\
\hline 202.5 & $6.55 \pm 0.41$ & $5.65 \pm 0.40$ & $5.17 \pm 0.41$ & $4.43 \pm 0.42$ & $3.09 \pm 0.31$ \\
\hline 217.5 & $7.91 \pm 0.45$ & $6.71 \pm 0.44$ & $5.95 \pm 0.45$ & $4.56 \pm 0.45$ & $4.48 \pm 0.36$ \\
\hline 232.5 & $8.60 \pm 0.48$ & $7.09 \pm 0.47$ & $6.30 \pm 0.48$ & $4.96 \pm 0.49$ & $4.53 \pm 0.41$ \\
\hline 247.5 & $8.45 \pm 0.48$ & $6.58 \pm 0.45$ & $7.35 \pm 0.49$ & $5.74 \pm 0.51$ & $5.68 \pm 0.49$ \\
\hline 262.5 & $9.14 \pm 0.50$ & $6.69 \pm 0.44$ & $5.67 \pm 0.42$ & $4.57 \pm 0.44$ & $4.27 \pm 0.38$ \\
\hline 277.5 & $9.48 \pm 0.51$ & $7.37 \pm 0.45$ & $5.58 \pm 0.41$ & $4.68 \pm 0.41$ & $3.96 \pm 0.34$ \\
\hline 292.5 & $10.94 \pm 0.54$ & $8.52 \pm 0.47$ & $6.79 \pm 0.44$ & $5.66 \pm 0.47$ & $4.76 \pm 0.39$ \\
\hline 307.5 & $11.68 \pm 0.54$ & $9.96 \pm 0.51$ & $7.98 \pm 0.51$ & $7.12 \pm 0.56$ & $5.75 \pm 0.52$ \\
\hline 322.5 & $11.76 \pm 0.54$ & $11.28 \pm 0.58$ & $9.73 \pm 0.63$ & $7.03 \pm 0.75$ & $6.65 \pm 0.85$ \\
\hline 337.5 & $13.06 \pm 0.57$ & $11.18 \pm 0.61$ & $9.78 \pm 0.81$ & $8.98 \pm 1.19$ & $9.72 \pm 1.88$ \\
\hline 352.5 & $13.55 \pm 0.58$ & $12.74 \pm 0.70$ & $9.79 \pm 0.98$ & $9.86 \pm 1.57$ & $11.50 \pm 3.25$ \\
\hline
\end{tabular}

TABLE 24: Unpolarized cross section for Kin 363 in $\mathrm{pb} / \mathrm{GeV}^{4}$ 


\begin{tabular}{|c|c|c|c|c|c|}
\hline & \multicolumn{5}{|c|}{$\sigma$} \\
\hline$\phi(\operatorname{deg})$ & $\begin{array}{l}\left\langle Q^{2}\right\rangle=4.532 \\
\left\langle x_{B}\right\rangle=0.369 \\
\left\langle t^{\prime}\right\rangle=-0.031\end{array}$ & $\begin{array}{l}\left\langle Q^{2}\right\rangle=4.550 \\
\left\langle x_{B}\right\rangle=0.370 \\
\left\langle t^{\prime}\right\rangle=-0.093\end{array}$ & $\begin{array}{l}\left\langle Q^{2}\right\rangle=4.574 \\
\left\langle x_{B}\right\rangle=0.372 \\
\left\langle t^{\prime}\right\rangle=-0.154\end{array}$ & $\begin{array}{l}\left\langle Q^{2}\right\rangle=4.585 \\
\left\langle x_{B}\right\rangle=0.373 \\
\left\langle t^{\prime}\right\rangle=-0.216\end{array}$ & $\begin{array}{l}\left\langle Q^{2}\right\rangle=4.568 \\
\left\langle x_{B}\right\rangle=0.371 \\
\left\langle t^{\prime}\right\rangle=-0.303\end{array}$ \\
\hline 7.5 & $0.83 \pm 0.64$ & $-0.32 \pm 0.82$ & $1.51 \pm 0.93$ & $1.51 \pm 1.47$ & $-3.90 \pm 2.62$ \\
\hline 22.5 & $1.11 \pm 0.65$ & $1.49 \pm 0.73$ & $1.89 \pm 0.88$ & $1.65 \pm 1.18$ & $-0.10 \pm 1.46$ \\
\hline 37.5 & $1.00 \pm 0.65$ & $1.29 \pm 0.65$ & $1.68 \pm 0.69$ & $1.71 \pm 0.79$ & $1.89 \pm 0.79$ \\
\hline 52.5 & $1.15 \pm 0.61$ & $1.70 \pm 0.59$ & $2.19 \pm 0.57$ & $1.46 \pm 0.65$ & $1.12 \pm 0.53$ \\
\hline 67.5 & $1.59 \pm 0.60$ & $2.78 \pm 0.56$ & $1.57 \pm 0.51$ & $1.87 \pm 0.52$ & $0.50 \pm 0.41$ \\
\hline 82.5 & $1.50 \pm 0.58$ & $2.84 \pm 0.50$ & $2.00 \pm 0.46$ & $1.27 \pm 0.49$ & $1.29 \pm 0.37$ \\
\hline 97.5 & $0.24 \pm 0.55$ & $1.54 \pm 0.50$ & $1.21 \pm 0.44$ & $1.13 \pm 0.47$ & $1.27 \pm 0.37$ \\
\hline 112.5 & $2.30 \pm 0.53$ & $1.96 \pm 0.49$ & $0.75 \pm 0.44$ & $1.56 \pm 0.47$ & $0.73 \pm 0.36$ \\
\hline 127.5 & $0.18 \pm 0.54$ & $0.95 \pm 0.51$ & $1.21 \pm 0.41$ & $1.46 \pm 0.46$ & $1.10 \pm 0.31$ \\
\hline 142.5 & $1.22 \pm 0.52$ & $1.05 \pm 0.48$ & $0.97 \pm 0.44$ & $0.38 \pm 0.44$ & $0.54 \pm 0.28$ \\
\hline 157.5 & $-0.12 \pm 0.50$ & $0.02 \pm 0.45$ & $0.40 \pm 0.40$ & $1.15 \pm 0.44$ & $0.62 \pm 0.28$ \\
\hline 172.5 & $0.70 \pm 0.47$ & $0.79 \pm 0.44$ & $0.68 \pm 0.39$ & $0.97 \pm 0.43$ & $0.05 \pm 0.28$ \\
\hline 187.5 & $0.59 \pm 0.54$ & $0.20 \pm 0.45$ & $-0.23 \pm 0.38$ & $-0.91 \pm 0.47$ & $-0.03 \pm 0.34$ \\
\hline 202.5 & $-0.43 \pm 0.52$ & $0.21 \pm 0.47$ & $-0.46 \pm 0.46$ & $0.27 \pm 0.50$ & $0.01 \pm 0.35$ \\
\hline 217.5 & $-1.26 \pm 0.56$ & $-0.66 \pm 0.51$ & $-1.04 \pm 0.50$ & $0.17 \pm 0.52$ & $-0.77 \pm 0.41$ \\
\hline 232.5 & $-1.37 \pm 0.60$ & $-1.24 \pm 0.55$ & $-1.42 \pm 0.54$ & $-2.37 \pm 0.57$ & $-1.07 \pm 0.47$ \\
\hline 247.5 & $-0.23 \pm 0.59$ & $-1.77 \pm 0.53$ & $-1.71 \pm 0.57$ & $-1.71 \pm 0.60$ & $-2.39 \pm 0.56$ \\
\hline 262.5 & $-2.00 \pm 0.61$ & $-1.38 \pm 0.53$ & $-1.14 \pm 0.48$ & $-0.68 \pm 0.51$ & $-1.66 \pm 0.44$ \\
\hline 277.5 & $-3.24 \pm 0.62$ & $-1.82 \pm 0.54$ & $-0.52 \pm 0.47$ & $-2.38 \pm 0.49$ & $-0.62 \pm 0.38$ \\
\hline 292.5 & $0.21 \pm 0.66$ & $-2.23 \pm 0.58$ & $-1.28 \pm 0.52$ & $-1.58 \pm 0.55$ & $-2.38 \pm 0.45$ \\
\hline 307.5 & $-1.96 \pm 0.66$ & $-2.01 \pm 0.63$ & $-2.03 \pm 0.59$ & $-2.74 \pm 0.64$ & $-1.88 \pm 0.57$ \\
\hline 322.5 & $-1.70 \pm 0.66$ & $-1.56 \pm 0.71$ & $-1.07 \pm 0.71$ & $-0.31 \pm 0.79$ & $-2.59 \pm 0.83$ \\
\hline 337.5 & $-1.31 \pm 0.70$ & $-0.01 \pm 0.72$ & $-0.78 \pm 0.83$ & $-0.31 \pm 1.13$ & $-2.26 \pm 1.65$ \\
\hline 352.5 & $1.04 \pm 0.75$ & $-1.55 \pm 0.84$ & $0.30 \pm 1.01$ & $-0.57 \pm 1.44$ & $-2.00 \pm 2.68$ \\
\hline
\end{tabular}

TABLE 25: Helicity-Dependent cross section for Kin 363 in pb/GeV ${ }^{4}$ 


\begin{tabular}{|c|c|c|c|c|c|}
\hline & \multicolumn{5}{|c|}{$\sigma$} \\
\hline$\phi(\operatorname{deg})$ & $\begin{array}{l}\left\langle Q^{2}\right\rangle=2.707 \\
\left\langle x_{B}\right\rangle=0.483 \\
\left\langle t^{\prime}\right\rangle=-0.022\end{array}$ & $\begin{array}{l}\left\langle Q^{2}\right\rangle=2.708 \\
\left\langle x_{B}\right\rangle=0.483 \\
\left\langle t^{\prime}\right\rangle=-0.064\end{array}$ & $\begin{array}{l}\left\langle Q^{2}\right\rangle=2.713 \\
\left\langle x_{B}\right\rangle=0.484 \\
\left\langle t^{\prime}\right\rangle=-0.107\end{array}$ & $\begin{array}{l}\left\langle Q^{2}\right\rangle=2.715 \\
\left\langle x_{B}\right\rangle=0.485 \\
\left\langle t^{\prime}\right\rangle=-0.150\end{array}$ & $\begin{array}{l}\left\langle Q^{2}\right\rangle=2.717 \\
\left\langle x_{B}\right\rangle=0.485 \\
\left\langle t^{\prime}\right\rangle=-0.210\end{array}$ \\
\hline 7.5 & $22.55 \pm 3.18$ & $28.33 \pm 4.29$ & $40.55 \pm 7.92$ & $59.50 \pm 31.05$ & nan \pm nan \\
\hline 22.5 & $28.38 \pm 3.38$ & $33.12 \pm 4.11$ & $29.43 \pm 6.36$ & $30.57 \pm 12.38$ & $-63.44 \pm 59.02$ \\
\hline 37.5 & $25.32 \pm 3.07$ & $26.06 \pm 3.71$ & $27.09 \pm 4.31$ & $5.51 \pm 4.77$ & $13.69 \pm 8.20$ \\
\hline 52.5 & $20.52 \pm 2.77$ & $25.00 \pm 3.23$ & $23.03 \pm 3.39$ & $11.30 \pm 2.93$ & $12.44 \pm 3.10$ \\
\hline 67.5 & $22.63 \pm 2.78$ & $22.65 \pm 2.91$ & $17.33 \pm 2.70$ & $13.20 \pm 2.81$ & $12.98 \pm 2.22$ \\
\hline 82.5 & $21.56 \pm 2.49$ & $16.27 \pm 2.40$ & $17.83 \pm 2.39$ & $15.03 \pm 2.53$ & $8.98 \pm 1.75$ \\
\hline 97.5 & $16.41 \pm 2.22$ & $11.07 \pm 2.18$ & $9.48 \pm 2.05$ & $14.82 \pm 2.27$ & $1.87 \pm 1.60$ \\
\hline 112.5 & $18.03 \pm 2.21$ & $16.19 \pm 2.17$ & $13.94 \pm 2.02$ & $10.91 \pm 1.97$ & $7.73 \pm 1.42$ \\
\hline 127.5 & $19.76 \pm 2.33$ & $12.67 \pm 1.96$ & $13.64 \pm 1.94$ & $9.60 \pm 1.91$ & $5.71 \pm 1.31$ \\
\hline 142.5 & $20.75 \pm 2.29$ & $13.85 \pm 1.97$ & $12.35 \pm 2.01$ & $10.84 \pm 2.17$ & $10.21 \pm 1.75$ \\
\hline 157.5 & $20.79 \pm 2.34$ & $12.85 \pm 1.98$ & $13.90 \pm 2.35$ & $13.60 \pm 2.60$ & $13.78 \pm 2.18$ \\
\hline 172.5 & $18.94 \pm 2.22$ & $12.04 \pm 1.90$ & $13.99 \pm 2.35$ & $14.06 \pm 2.68$ & $14.34 \pm 2.47$ \\
\hline 187.5 & $17.68 \pm 2.27$ & $14.87 \pm 2.04$ & $13.07 \pm 2.20$ & $13.70 \pm 2.35$ & $12.24 \pm 1.90$ \\
\hline 202.5 & $16.95 \pm 2.28$ & $15.16 \pm 2.14$ & $12.08 \pm 2.03$ & $12.12 \pm 2.04$ & $7.58 \pm 1.35$ \\
\hline 217.5 & $18.45 \pm 2.27$ & $17.41 \pm 2.15$ & $13.28 \pm 2.03$ & $9.51 \pm 1.85$ & $6.75 \pm 1.18$ \\
\hline 232.5 & $19.69 \pm 2.40$ & $16.24 \pm 2.20$ & $15.83 \pm 2.15$ & $11.70 \pm 2.03$ & $6.64 \pm 1.27$ \\
\hline 247.5 & $19.89 \pm 2.39$ & $15.85 \pm 2.37$ & $14.25 \pm 2.29$ & $13.61 \pm 2.33$ & $6.86 \pm 1.44$ \\
\hline 262.5 & $21.46 \pm 2.46$ & $25.25 \pm 2.63$ & $13.41 \pm 2.61$ & $17.85 \pm 2.93$ & $8.21 \pm 1.69$ \\
\hline 277.5 & $17.26 \pm 2.45$ & $19.26 \pm 2.73$ & $20.38 \pm 2.98$ & $18.27 \pm 3.20$ & $-9.52 \pm 2.39$ \\
\hline 292.5 & $23.50 \pm 2.66$ & $21.10 \pm 2.78$ & $28.84 \pm 3.43$ & $20.72 \pm 3.82$ & $-4.73 \pm 3.24$ \\
\hline 307.5 & $21.87 \pm 2.77$ & $23.51 \pm 3.08$ & $20.67 \pm 3.42$ & $24.22 \pm 3.85$ & $16.31 \pm 4.63$ \\
\hline 322.5 & $26.76 \pm 2.95$ & $22.93 \pm 3.08$ & $20.67 \pm 3.62$ & $15.00 \pm 4.71$ & $15.90 \pm 11.09$ \\
\hline 337.5 & $26.47 \pm 3.09$ & $27.67 \pm 3.29$ & $19.53 \pm 5.17$ & $16.83 \pm 10.89$ & $49.44 \pm 67.41$ \\
\hline 352.5 & $26.56 \pm 3.17$ & $23.28 \pm 3.84$ & $27.99 \pm 6.93$ & $31.21 \pm 24.23$ & nan \pm nan \\
\hline
\end{tabular}

TABLE 26: Unpolarized cross section for Kin 481 in $\mathrm{pb} / \mathrm{GeV}^{4}$ 


\begin{tabular}{|c|c|c|c|c|c|}
\hline & \multicolumn{5}{|c|}{$\sigma$} \\
\hline$\phi(\mathrm{deg})$ & $\begin{array}{l}\left\langle Q^{2}\right\rangle=2.707 \\
\left\langle x_{B}\right\rangle=0.483 \\
\left\langle t^{\prime}\right\rangle=-0.022\end{array}$ & $\begin{array}{l}\left\langle Q^{2}\right\rangle=2.708 \\
\left\langle x_{B}\right\rangle=0.483 \\
\left\langle t^{\prime}\right\rangle=-0.064\end{array}$ & $\begin{array}{l}\left\langle Q^{2}\right\rangle=2.713 \\
\left\langle x_{B}\right\rangle=0.484 \\
\left\langle t^{\prime}\right\rangle=-0.107\end{array}$ & $\begin{array}{l}\left\langle Q^{2}\right\rangle=2.715 \\
\left\langle x_{B}\right\rangle=0.485 \\
\left\langle t^{\prime}\right\rangle=-0.150\end{array}$ & $\begin{array}{l}\left\langle Q^{2}\right\rangle=2.717 \\
\left\langle x_{B}\right\rangle=0.485 \\
\left\langle t^{\prime}\right\rangle=-0.210\end{array}$ \\
\hline 7.5 & $\begin{array}{l}-12.05 \\
-11.27\end{array}$ & $-7.91 \pm 4.79$ & $13.93 \pm 9.02$ & $-10.63 \pm 44.01$ & nan $\pm \inf$ \\
\hline 22.5 & $-1.71 \pm-4.82$ & $2.66 \pm 4.81$ & $1.74 \pm 8.26$ & $17.81 \pm 19.54$ & $-11.98 \pm 55.46$ \\
\hline 37.5 & $-0.29 \pm-0.87$ & $-1.80 \pm 4.42$ & $5.29 \pm 6.77$ & $-5.66 \pm 9.62$ & $1.79 \pm 8.47$ \\
\hline 52.5 & $0.32 \pm 1.06$ & $7.17 \pm 3.85$ & $0.77 \pm 4.52$ & $-7.11 \pm 9.50$ & $5.28 \pm 3.60$ \\
\hline 67.5 & $0.59 \pm 2.19$ & $7.70 \pm 3.45$ & $4.21 \pm 2.91$ & $4.03 \pm-3.31$ & $5.83 \pm 2.68$ \\
\hline 82.5 & $2.71 \pm 2.38$ & $7.66 \pm 2.84$ & $-1.87 \pm 1.81$ & $2.37 \pm 2.14$ & $2.50 \pm 2.18$ \\
\hline 97.5 & $1.67 \pm 2.37$ & $4.26 \pm 2.60$ & $1.13 \pm 0.85$ & $3.37 \pm 2.45$ & $-2.38 \pm 1.94$ \\
\hline 112.5 & $1.76 \pm 2.46$ & $3.97 \pm 2.59$ & $-0.00 \pm-0.00$ & $6.11 \pm 2.30$ & $1.27 \pm 1.61$ \\
\hline 127.5 & $1.29 \pm 2.64$ & $1.55 \pm 2.32$ & $0.22 \pm-0.82$ & $2.19 \pm 2.33$ & $0.76 \pm 1.32$ \\
\hline 142.5 & $3.69 \pm 2.55$ & $-0.11 \pm 2.28$ & $-3.18 \pm-1.70$ & $1.31 \pm 2.79$ & $2.90 \pm 1.53$ \\
\hline 157.5 & $-0.64 \pm 2.54$ & $1.89 \pm 2.25$ & $0.15 \pm-2.98$ & $3.38 \pm 3.44$ & $0.27 \pm 1.54$ \\
\hline 172.5 & $0.09 \pm 2.15$ & $0.99 \pm 2.12$ & $-3.15 \pm-3.11$ & $3.78 \pm 3.39$ & $2.50 \pm 1.50$ \\
\hline 187.5 & $0.42 \pm 3.17$ & $-1.54 \pm 2.66$ & $-6.56 \pm-3.79$ & $-1.69 \pm 3.13$ & $0.51 \pm 1.14$ \\
\hline 202.5 & $-3.15 \pm 2.75$ & $2.25 \pm 2.59$ & $-0.33 \pm-3.80$ & $-2.86 \pm 2.75$ & $-1.00 \pm 0.96$ \\
\hline 217.5 & $-3.74 \pm 2.70$ & $-0.99 \pm 2.55$ & $-2.53 \pm-1.99$ & $-3.69 \pm 2.46$ & $-1.05 \pm 1.03$ \\
\hline 232.5 & $-0.78 \pm 2.82$ & $-4.55 \pm 2.62$ & $0.36 \pm-1.04$ & $-2.82 \pm 2.61$ & $-1.55 \pm 1.28$ \\
\hline 247.5 & $-0.46 \pm 2.74$ & $0.52 \pm 2.79$ & $0.00 \pm-0.01$ & $-0.10 \pm 2.87$ & $-0.52 \pm 1.67$ \\
\hline 262.5 & $0.37 \pm 2.81$ & $-8.15 \pm 3.11$ & $1.41 \pm 1.05$ & $-8.49 \pm 3.48$ & $-1.12 \pm 2.19$ \\
\hline 277.5 & $0.65 \pm 2.56$ & $-2.66 \pm 3.23$ & $-3.87 \pm 2.17$ & $-5.89 \pm 3.21$ & $-33.05 \pm 3.21$ \\
\hline 292.5 & $-0.81 \pm 2.32$ & $-2.24 \pm 3.36$ & $3.71 \pm 3.22$ & $11.97 \pm-32.65$ & $-35.91 \pm 4.28$ \\
\hline 307.5 & $0.78 \pm 1.36$ & $-3.60 \pm 3.80$ & $-0.81 \pm 4.05$ & $-5.81 \pm 8.85$ & $-4.08 \pm 5.76$ \\
\hline 322.5 & $-1.18 \pm-1.24$ & $-4.53 \pm 3.82$ & $2.00 \pm 4.74$ & $14.03 \pm 8.89$ & $1.69 \pm 12.56$ \\
\hline 337.5 & $16.26 \pm-6.99$ & $3.21 \pm 4.05$ & $-12.21 \pm 6.54$ & $20.64 \pm 16.42$ & $9.14 \pm 67.42$ \\
\hline 352.5 & $26.92 \pm-65.67$ & $-2.08 \pm 5.15$ & $5.70 \pm 9.77$ & $43.84 \pm 38.23$ & $\inf \pm$-inf \\
\hline
\end{tabular}

TABLE 27: Helicity-Dependent cross section for Kin 481 in pb/GeV ${ }^{4}$ 


\begin{tabular}{|c|c|c|c|c|c|}
\hline & \multicolumn{5}{|c|}{$\sigma$} \\
\hline$\phi(\operatorname{deg})$ & $\begin{array}{l}\left\langle Q^{2}\right\rangle=4.497 \\
\left\langle x_{B}\right\rangle=0.497 \\
\left\langle t^{\prime}\right\rangle=-0.031\end{array}$ & $\begin{array}{l}\left\langle Q^{2}\right\rangle=4.528 \\
\left\langle x_{B}\right\rangle=0.501 \\
\left\langle t^{\prime}\right\rangle=-0.093\end{array}$ & $\begin{array}{l}\left\langle Q^{2}\right\rangle=4.558 \\
\left\langle x_{B}\right\rangle=0.504 \\
\left\langle t^{\prime}\right\rangle=-0.154\end{array}$ & $\begin{array}{l}\left\langle Q^{2}\right\rangle=4.573 \\
\left\langle x_{B}\right\rangle=0.506 \\
\left\langle t^{\prime}\right\rangle=-0.216\end{array}$ & $\begin{array}{l}\left\langle Q^{2}\right\rangle=4.593 \\
\left\langle x_{B}\right\rangle=0.508 \\
\left\langle t^{\prime}\right\rangle=-0.305\end{array}$ \\
\hline 7.5 & $2.43 \pm 0.26$ & $1.54 \pm 0.39$ & $3.05 \pm 1.08$ & $1.49 \pm 1.85$ & nan \pm nan \\
\hline 22.5 & $3.36 \pm 0.28$ & $2.42 \pm 0.34$ & $2.74 \pm 0.72$ & $0.66 \pm 1.13$ & $0.00 \pm 4.31$ \\
\hline 37.5 & $3.37 \pm 0.27$ & $2.57 \pm 0.30$ & $2.10 \pm 0.43$ & $1.95 \pm 0.53$ & $-0.29 \pm 0.83$ \\
\hline 52.5 & $3.37 \pm 0.27$ & $2.42 \pm 0.25$ & $1.53 \pm 0.26$ & $1.27 \pm 0.32$ & $1.00 \pm 0.31$ \\
\hline 67.5 & $2.97 \pm 0.25$ & $1.89 \pm 0.21$ & $1.97 \pm 0.22$ & $1.24 \pm 0.22$ & $0.64 \pm 0.20$ \\
\hline 82.5 & $3.08 \pm 0.25$ & $2.22 \pm 0.22$ & $1.84 \pm 0.21$ & $1.69 \pm 0.23$ & $1.02 \pm 0.17$ \\
\hline 97.5 & $2.95 \pm 0.25$ & $1.92 \pm 0.21$ & $1.80 \pm 0.21$ & $1.40 \pm 0.20$ & $0.98 \pm 0.16$ \\
\hline 112.5 & $2.61 \pm 0.24$ & $2.25 \pm 0.22$ & $1.86 \pm 0.22$ & $1.17 \pm 0.18$ & $1.24 \pm 0.15$ \\
\hline 127.5 & $2.69 \pm 0.25$ & $1.88 \pm 0.21$ & $2.10 \pm 0.22$ & $1.42 \pm 0.19$ & $1.18 \pm 0.14$ \\
\hline 142.5 & $2.78 \pm 0.25$ & $2.03 \pm 0.21$ & $1.75 \pm 0.21$ & $1.23 \pm 0.19$ & $1.30 \pm 0.15$ \\
\hline 157.5 & $3.17 \pm 0.26$ & $1.95 \pm 0.21$ & $2.40 \pm 0.25$ & $1.67 \pm 0.22$ & $1.79 \pm 0.20$ \\
\hline 172.5 & $2.64 \pm 0.24$ & $1.45 \pm 0.20$ & $2.18 \pm 0.24$ & $1.39 \pm 0.21$ & $1.43 \pm 0.18$ \\
\hline 187.5 & $2.60 \pm 0.24$ & $2.17 \pm 0.23$ & $2.18 \pm 0.25$ & $1.87 \pm 0.22$ & $1.40 \pm 0.15$ \\
\hline 202.5 & $2.98 \pm 0.26$ & $2.65 \pm 0.25$ & $1.77 \pm 0.24$ & $1.58 \pm 0.21$ & $1.34 \pm 0.14$ \\
\hline 217.5 & $2.46 \pm 0.23$ & $1.92 \pm 0.22$ & $1.89 \pm 0.23$ & $1.65 \pm 0.22$ & $1.63 \pm 0.15$ \\
\hline 232.5 & $2.07 \pm 0.22$ & $2.38 \pm 0.23$ & $1.92 \pm 0.24$ & $1.87 \pm 0.24$ & $1.65 \pm 0.16$ \\
\hline 247.5 & $2.61 \pm 0.24$ & $2.09 \pm 0.23$ & $2.84 \pm 0.29$ & $1.71 \pm 0.25$ & $1.79 \pm 0.19$ \\
\hline 262.5 & $3.45 \pm 0.26$ & $2.52 \pm 0.25$ & $2.45 \pm 0.28$ & $2.32 \pm 0.28$ & $1.60 \pm 0.21$ \\
\hline 277.5 & $3.25 \pm 0.25$ & $2.68 \pm 0.24$ & $2.45 \pm 0.25$ & $2.03 \pm 0.25$ & $1.72 \pm 0.22$ \\
\hline 292.5 & $3.31 \pm 0.25$ & $2.52 \pm 0.23$ & $2.57 \pm 0.25$ & $1.82 \pm 0.26$ & $1.37 \pm 0.23$ \\
\hline 307.5 & $3.09 \pm 0.25$ & $2.42 \pm 0.24$ & $2.14 \pm 0.28$ & $1.74 \pm 0.36$ & $2.03 \pm 0.41$ \\
\hline 322.5 & $3.19 \pm 0.25$ & $2.32 \pm 0.27$ & $1.76 \pm 0.41$ & $1.65 \pm 0.61$ & $1.73 \pm 0.99$ \\
\hline 337.5 & $3.32 \pm 0.27$ & $2.46 \pm 0.36$ & $3.10 \pm 0.75$ & $2.33 \pm 1.20$ & $-0.84 \pm 1.74$ \\
\hline 352.5 & $2.79 \pm 0.26$ & $2.69 \pm 0.42$ & $3.84 \pm 1.12$ & $-1.62 \pm 1.70$ & nan \pm nan \\
\hline
\end{tabular}

TABLE 28: Unpolarized cross section for Kin 482 in pb/GeV $V^{4}$ 


\begin{tabular}{|c|c|c|c|c|c|}
\hline & \multicolumn{5}{|c|}{$\sigma$} \\
\hline$\phi(\operatorname{deg})$ & $\begin{array}{l}\left\langle Q^{2}\right\rangle=4.497 \\
\left\langle x_{B}\right\rangle=0.497 \\
\left\langle t^{\prime}\right\rangle=-0.031\end{array}$ & $\begin{array}{l}\left\langle Q^{2}\right\rangle=4.528 \\
\left\langle x_{B}\right\rangle=0.501 \\
\left\langle t^{\prime}\right\rangle=-0.093\end{array}$ & $\begin{array}{l}\left\langle Q^{2}\right\rangle=4.558 \\
\left\langle x_{B}\right\rangle=0.504 \\
\left\langle t^{\prime}\right\rangle=-0.154\end{array}$ & $\begin{array}{l}\left\langle Q^{2}\right\rangle=4.573 \\
\left\langle x_{B}\right\rangle=0.506 \\
\left\langle t^{\prime}\right\rangle=-0.216\end{array}$ & $\begin{array}{l}\left\langle Q^{2}\right\rangle=4.593 \\
\left\langle x_{B}\right\rangle=0.508 \\
\left\langle t^{\prime}\right\rangle=-0.305\end{array}$ \\
\hline 7.5 & $0.21 \pm 0.40$ & $0.72 \pm 0.43$ & $0.38 \pm 1.22$ & $2.12 \pm 1.89$ & nan \pm nan \\
\hline 22.5 & $0.23 \pm 0.41$ & $0.45 \pm 0.38$ & $0.04 \pm 0.79$ & $0.68 \pm 1.30$ & $0.00 \pm 2.83$ \\
\hline 37.5 & $0.24 \pm 0.37$ & $0.70 \pm 0.35$ & $0.32 \pm 0.48$ & $0.91 \pm 0.69$ & $1.22 \pm 0.87$ \\
\hline 52.5 & $0.08 \pm 0.35$ & $0.36 \pm 0.29$ & $0.27 \pm 0.30$ & $0.77 \pm 0.42$ & $0.72 \pm 0.36$ \\
\hline 67.5 & $0.28 \pm 0.32$ & $0.44 \pm 0.26$ & $0.49 \pm 0.26$ & $0.74 \pm 0.29$ & $0.23 \pm 0.24$ \\
\hline 82.5 & $0.23 \pm 0.30$ & $0.26 \pm 0.26$ & $0.51 \pm 0.25$ & $0.39 \pm 0.28$ & $0.26 \pm 0.20$ \\
\hline 97.5 & $0.61 \pm 0.29$ & $0.45 \pm 0.25$ & $0.38 \pm 0.24$ & $0.21 \pm 0.24$ & $0.25 \pm 0.19$ \\
\hline 112.5 & $0.19 \pm 0.27$ & $0.71 \pm 0.27$ & $0.63 \pm 0.24$ & $0.37 \pm 0.21$ & $0.03 \pm 0.17$ \\
\hline 127.5 & $0.09 \pm 0.27$ & $0.90 \pm 0.25$ & $0.38 \pm 0.24$ & $0.45 \pm 0.21$ & $0.49 \pm 0.15$ \\
\hline 142.5 & $-0.32 \pm 0.26$ & $0.31 \pm 0.25$ & $0.32 \pm 0.22$ & $0.03 \pm 0.20$ & $0.52 \pm 0.17$ \\
\hline 157.5 & $-0.01 \pm 0.28$ & $0.41 \pm 0.26$ & $0.76 \pm 0.26$ & $0.14 \pm 0.23$ & $0.23 \pm 0.22$ \\
\hline 172.5 & $-0.13 \pm 0.28$ & $0.34 \pm 0.25$ & $0.08 \pm 0.26$ & $-0.03 \pm 0.23$ & $0.10 \pm 0.22$ \\
\hline 187.5 & $0.10 \pm 0.21$ & $-0.12 \pm 0.26$ & $-0.02 \pm 0.25$ & $0.03 \pm 0.22$ & $-0.07 \pm 0.16$ \\
\hline 202.5 & $0.37 \pm 0.25$ & $0.00 \pm 0.29$ & $0.29 \pm 0.25$ & $-0.37 \pm 0.21$ & $-0.13 \pm 0.15$ \\
\hline 217.5 & $0.14 \pm 0.22$ & $-0.42 \pm 0.25$ & $-0.06 \pm 0.25$ & $-0.28 \pm 0.23$ & $-0.36 \pm 0.17$ \\
\hline 232.5 & $-0.46 \pm 0.22$ & $0.35 \pm 0.27$ & $-0.24 \pm 0.26$ & $-0.40 \pm 0.26$ & $-0.59 \pm 0.18$ \\
\hline 247.5 & $-0.29 \pm 0.26$ & $-0.21 \pm 0.27$ & $-0.56 \pm 0.31$ & $-0.20 \pm 0.28$ & $-0.36 \pm 0.22$ \\
\hline 262.5 & $-0.42 \pm 0.29$ & $-0.37 \pm 0.29$ & $-0.54 \pm 0.32$ & $-0.42 \pm 0.34$ & $-0.26 \pm 0.25$ \\
\hline 277.5 & $-0.46 \pm 0.30$ & $-1.14 \pm 0.29$ & $-1.09 \pm 0.29$ & $-0.98 \pm 0.32$ & $-0.04 \pm 0.27$ \\
\hline 292.5 & $-0.89 \pm 0.31$ & $-0.27 \pm 0.27$ & $-0.97 \pm 0.30$ & $-0.65 \pm 0.34$ & $-0.41 \pm 0.29$ \\
\hline 307.5 & $-0.37 \pm 0.32$ & $0.01 \pm 0.28$ & $-0.41 \pm 0.32$ & $-1.23 \pm 0.47$ & $-0.84 \pm 0.48$ \\
\hline 322.5 & $0.03 \pm 0.33$ & $0.18 \pm 0.30$ & $-0.86 \pm 0.45$ & $-0.63 \pm 0.75$ & $-2.18 \pm 1.06$ \\
\hline 337.5 & $-0.25 \pm 0.37$ & $-0.31 \pm 0.38$ & $-1.01 \pm 0.78$ & $2.89 \pm 1.42$ & $-0.82 \pm 1.70$ \\
\hline 352.5 & $0.09 \pm 0.34$ & $-0.24 \pm 0.40$ & $-1.06 \pm 1.08$ & $-0.34 \pm 1.63$ & nan \pm nan \\
\hline
\end{tabular}

TABLE 29: Helicity-Dependent cross section for Kin 482 in pb/GeV 


\begin{tabular}{|c|c|c|c|c|c|}
\hline & \multicolumn{5}{|c|}{$\sigma$} \\
\hline$\phi(\operatorname{deg})$ & $\begin{array}{l}\left\langle Q^{2}\right\rangle=5.331 \\
\left\langle x_{B}\right\rangle=0.482 \\
\left\langle t^{\prime}\right\rangle=-0.030\end{array}$ & $\begin{array}{l}\left\langle Q^{2}\right\rangle=5.339 \\
\left\langle x_{B}\right\rangle=0.483 \\
\left\langle t^{\prime}\right\rangle=-0.088\end{array}$ & $\begin{array}{l}\left\langle Q^{2}\right\rangle=5.360 \\
\left\langle x_{B}\right\rangle=0.485 \\
\left\langle t^{\prime}\right\rangle=-0.147\end{array}$ & $\begin{array}{l}\left\langle Q^{2}\right\rangle=5.371 \\
\left\langle x_{B}\right\rangle=0.486 \\
\left\langle t^{\prime}\right\rangle=-0.206\end{array}$ & $\begin{array}{l}\left\langle Q^{2}\right\rangle=5.379 \\
\left\langle x_{B}\right\rangle=0.486 \\
\left\langle t^{\prime}\right\rangle=-0.291\end{array}$ \\
\hline 7.5 & $3.84 \pm 0.28$ & $3.40 \pm 0.32$ & $4.41 \pm 0.67$ & $0.30 \pm 1.15$ & $13.28 \pm 10.92$ \\
\hline 22.5 & $4.01 \pm 0.28$ & $3.53 \pm 0.30$ & $2.28 \pm 0.43$ & $2.24 \pm 0.75$ & $1.05 \pm 1.77$ \\
\hline 37.5 & $3.59 \pm 0.26$ & $2.47 \pm 0.24$ & $2.87 \pm 0.32$ & $2.06 \pm 0.42$ & $1.44 \pm 0.56$ \\
\hline 52.5 & $3.66 \pm 0.27$ & $2.93 \pm 0.25$ & $2.57 \pm 0.24$ & $2.22 \pm 0.28$ & $1.33 \pm 0.25$ \\
\hline 67.5 & $3.56 \pm 0.27$ & $2.75 \pm 0.24$ & $2.23 \pm 0.22$ & $1.85 \pm 0.22$ & $1.40 \pm 0.19$ \\
\hline 82.5 & $2.92 \pm 0.24$ & $2.78 \pm 0.24$ & $1.99 \pm 0.20$ & $1.63 \pm 0.19$ & $1.47 \pm 0.16$ \\
\hline 97.5 & $3.09 \pm 0.25$ & $2.20 \pm 0.22$ & $1.79 \pm 0.19$ & $1.41 \pm 0.18$ & $1.07 \pm 0.13$ \\
\hline 112.5 & $3.19 \pm 0.25$ & $2.50 \pm 0.22$ & $1.42 \pm 0.17$ & $1.45 \pm 0.17$ & $1.04 \pm 0.12$ \\
\hline 127.5 & $2.89 \pm 0.24$ & $2.04 \pm 0.20$ & $1.58 \pm 0.17$ & $1.62 \pm 0.17$ & $1.04 \pm 0.11$ \\
\hline 142.5 & $2.79 \pm 0.23$ & $2.13 \pm 0.19$ & $1.95 \pm 0.19$ & $1.31 \pm 0.16$ & $0.83 \pm 0.10$ \\
\hline 157.5 & $2.88 \pm 0.23$ & $1.97 \pm 0.19$ & $1.54 \pm 0.18$ & $1.46 \pm 0.17$ & $0.92 \pm 0.11$ \\
\hline 172.5 & $2.92 \pm 0.23$ & $1.70 \pm 0.19$ & $1.90 \pm 0.20$ & $1.53 \pm 0.20$ & $1.24 \pm 0.14$ \\
\hline 187.5 & $2.86 \pm 0.23$ & $2.42 \pm 0.22$ & $1.90 \pm 0.21$ & $2.11 \pm 0.26$ & $1.76 \pm 0.19$ \\
\hline 202.5 & $2.85 \pm 0.24$ & $2.04 \pm 0.21$ & $1.76 \pm 0.21$ & $1.93 \pm 0.27$ & $2.04 \pm 0.21$ \\
\hline 217.5 & $2.63 \pm 0.22$ & $2.42 \pm 0.22$ & $1.96 \pm 0.22$ & $1.62 \pm 0.22$ & $1.17 \pm 0.14$ \\
\hline 232.5 & $2.96 \pm 0.23$ & $2.01 \pm 0.21$ & $1.97 \pm 0.22$ & $1.81 \pm 0.23$ & $1.65 \pm 0.15$ \\
\hline 247.5 & $2.97 \pm 0.23$ & $2.82 \pm 0.24$ & $2.04 \pm 0.21$ & $1.92 \pm 0.22$ & $1.50 \pm 0.16$ \\
\hline 262.5 & $3.37 \pm 0.25$ & $3.04 \pm 0.24$ & $2.31 \pm 0.22$ & $2.16 \pm 0.22$ & $1.51 \pm 0.16$ \\
\hline 277.5 & $3.00 \pm 0.25$ & $3.00 \pm 0.24$ & $2.26 \pm 0.23$ & $2.17 \pm 0.23$ & $1.61 \pm 0.17$ \\
\hline 292.5 & $3.46 \pm 0.25$ & $3.11 \pm 0.24$ & $2.62 \pm 0.24$ & $2.24 \pm 0.25$ & $2.01 \pm 0.21$ \\
\hline 307.5 & $3.53 \pm 0.26$ & $3.17 \pm 0.25$ & $2.88 \pm 0.26$ & $2.84 \pm 0.30$ & $2.44 \pm 0.29$ \\
\hline 322.5 & $3.54 \pm 0.26$ & $3.26 \pm 0.26$ & $3.11 \pm 0.33$ & $3.00 \pm 0.46$ & $1.94 \pm 0.60$ \\
\hline 337.5 & $4.50 \pm 0.29$ & $3.49 \pm 0.31$ & $2.91 \pm 0.45$ & $3.48 \pm 0.87$ & $5.36 \pm 2.42$ \\
\hline 352.5 & $4.08 \pm 0.29$ & $3.27 \pm 0.32$ & $3.87 \pm 0.59$ & $3.18 \pm 1.55$ & $0.00 \pm 4.83$ \\
\hline
\end{tabular}

TABLE 30: Unpolarized cross section for Kin 483 in pb/GeV $V^{4}$ 


\begin{tabular}{|c|c|c|c|c|c|}
\hline & \multicolumn{5}{|c|}{$\sigma$} \\
\hline$\phi(\operatorname{deg})$ & $\begin{array}{l}\left\langle Q^{2}\right\rangle=5.331 \\
\left\langle x_{B}\right\rangle=0.482 \\
\left\langle t^{\prime}\right\rangle=-0.030\end{array}$ & $\begin{array}{l}\left\langle Q^{2}\right\rangle=5.339 \\
\left\langle x_{B}\right\rangle=0.483 \\
\left\langle t^{\prime}\right\rangle=-0.088\end{array}$ & $\begin{array}{l}\left\langle Q^{2}\right\rangle=5.360 \\
\left\langle x_{B}\right\rangle=0.485 \\
\left\langle t^{\prime}\right\rangle=-0.147\end{array}$ & $\begin{array}{l}\left\langle Q^{2}\right\rangle=5.371 \\
\left\langle x_{B}\right\rangle=0.486 \\
\left\langle t^{\prime}\right\rangle=-0.206\end{array}$ & $\begin{array}{l}\left\langle Q^{2}\right\rangle=5.379 \\
\left\langle x_{B}\right\rangle=0.486 \\
\left\langle t^{\prime}\right\rangle=-0.291\end{array}$ \\
\hline 7.5 & $-0.05 \pm 0.32$ & $-0.04 \pm 0.33$ & $0.72 \pm 0.71$ & $0.34 \pm 1.01$ & $-6.67 \pm 8.77$ \\
\hline 22.5 & $0.44 \pm 0.32$ & $0.93 \pm 0.32$ & $0.78 \pm 0.47$ & $0.91 \pm 0.74$ & $1.69 \pm 1.55$ \\
\hline 37.5 & $0.41 \pm 0.30$ & $0.75 \pm 0.26$ & $0.62 \pm 0.37$ & $0.76 \pm 0.45$ & $0.56 \pm 0.56$ \\
\hline 52.5 & $0.37 \pm 0.32$ & $0.39 \pm 0.27$ & $0.99 \pm 0.29$ & $0.52 \pm 0.33$ & $0.18 \pm 0.28$ \\
\hline 67.5 & $0.30 \pm 0.32$ & $0.17 \pm 0.26$ & $0.93 \pm 0.26$ & $0.76 \pm 0.26$ & $0.48 \pm 0.22$ \\
\hline 82.5 & $0.39 \pm 0.29$ & $0.89 \pm 0.27$ & $0.54 \pm 0.24$ & $-0.04 \pm 0.23$ & $0.21 \pm 0.19$ \\
\hline 97.5 & $0.77 \pm 0.29$ & $-0.13 \pm 0.25$ & $0.75 \pm 0.23$ & $0.49 \pm 0.20$ & $0.12 \pm 0.15$ \\
\hline 112.5 & $-0.05 \pm 0.30$ & $0.61 \pm 0.24$ & $0.33 \pm 0.19$ & $0.38 \pm 0.19$ & $0.27 \pm 0.13$ \\
\hline 127.5 & $0.48 \pm 0.29$ & $0.40 \pm 0.22$ & $0.17 \pm 0.20$ & $0.29 \pm 0.19$ & $0.21 \pm 0.11$ \\
\hline 142.5 & $0.27 \pm 0.28$ & $0.60 \pm 0.22$ & $0.33 \pm 0.21$ & $-0.04 \pm 0.17$ & $0.06 \pm 0.10$ \\
\hline 157.5 & $0.03 \pm 0.28$ & $0.46 \pm 0.22$ & $0.00 \pm 0.19$ & $0.12 \pm 0.19$ & $0.01 \pm 0.11$ \\
\hline 172.5 & $0.04 \pm 0.29$ & $-0.15 \pm 0.21$ & $0.09 \pm 0.22$ & $0.45 \pm 0.21$ & $0.01 \pm 0.13$ \\
\hline 187.5 & $-0.09 \pm 0.27$ & $-0.02 \pm 0.25$ & $-0.29 \pm 0.24$ & $-0.12 \pm 0.30$ & $0.30 \pm 0.20$ \\
\hline 202.5 & $-0.39 \pm 0.28$ & $0.19 \pm 0.23$ & $-0.21 \pm 0.22$ & $-0.62 \pm 0.30$ & $-0.29 \pm 0.21$ \\
\hline 217.5 & $0.04 \pm 0.26$ & $-0.54 \pm 0.24$ & $-0.47 \pm 0.24$ & $-0.32 \pm 0.24$ & $0.08 \pm 0.15$ \\
\hline 232.5 & $-0.14 \pm 0.28$ & $-0.14 \pm 0.24$ & $-0.59 \pm 0.25$ & $-0.53 \pm 0.26$ & $-0.20 \pm 0.16$ \\
\hline 247.5 & $-0.72 \pm 0.28$ & $-0.32 \pm 0.27$ & $-0.67 \pm 0.24$ & $-0.59 \pm 0.25$ & $-0.26 \pm 0.18$ \\
\hline 262.5 & $-0.05 \pm 0.29$ & $-0.65 \pm 0.27$ & $-0.22 \pm 0.26$ & $-0.69 \pm 0.25$ & $-0.36 \pm 0.18$ \\
\hline 277.5 & $-0.09 \pm 0.29$ & $-0.12 \pm 0.27$ & $-0.51 \pm 0.27$ & $-0.48 \pm 0.27$ & $-0.39 \pm 0.19$ \\
\hline 292.5 & $-0.39 \pm 0.29$ & $-0.33 \pm 0.26$ & $-0.22 \pm 0.29$ & $-0.49 \pm 0.30$ & $-0.58 \pm 0.24$ \\
\hline 307.5 & $-0.10 \pm 0.30$ & $-0.30 \pm 0.27$ & $-0.80 \pm 0.32$ & $-1.34 \pm 0.36$ & $-0.30 \pm 0.32$ \\
\hline 322.5 & $-0.22 \pm 0.30$ & $-0.33 \pm 0.28$ & $-0.74 \pm 0.38$ & $-0.36 \pm 0.50$ & $-0.42 \pm 0.60$ \\
\hline 337.5 & $0.03 \pm 0.33$ & $0.07 \pm 0.32$ & $-0.72 \pm 0.49$ & $0.55 \pm 0.85$ & $0.44 \pm 2.16$ \\
\hline 352.5 & $0.09 \pm 0.31$ & $0.18 \pm 0.32$ & $-0.25 \pm 0.61$ & $-1.59 \pm 1.36$ & $0.00 \pm 4.01$ \\
\hline
\end{tabular}

TABLE 31: Helicity-Dependent cross section for Kin 483 in pb/GeV $V^{4}$ 


\begin{tabular}{|c|c|c|c|c|c|}
\hline & \multicolumn{5}{|c|}{$\sigma$} \\
\hline$\phi(\operatorname{deg})$ & $\begin{array}{l}\left\langle Q^{2}\right\rangle=7.044 \\
\left\langle x_{B}\right\rangle=0.494 \\
\left\langle t^{\prime}\right\rangle=-0.043\end{array}$ & $\begin{array}{l}\left\langle Q^{2}\right\rangle=7.093 \\
\left\langle x_{B}\right\rangle=0.498 \\
\left\langle t^{\prime}\right\rangle=-0.127\end{array}$ & $\begin{array}{l}\left\langle Q^{2}\right\rangle=7.115 \\
\left\langle x_{B}\right\rangle=0.499 \\
\left\langle t^{\prime}\right\rangle=-0.212\end{array}$ & $\begin{array}{l}\left\langle Q^{2}\right\rangle=7.106 \\
\left\langle x_{B}\right\rangle=0.499 \\
\left\langle t^{\prime}\right\rangle=-0.297\end{array}$ & $\begin{array}{l}\left\langle Q^{2}\right\rangle=7.102 \\
\left\langle x_{B}\right\rangle=0.498 \\
\left\langle t^{\prime}\right\rangle=-0.457\end{array}$ \\
\hline 7.5 & $1.88 \pm 0.13$ & $1.69 \pm 0.24$ & $0.97 \pm 0.84$ & nan \pm nan & nan $\pm \inf$ \\
\hline 22.5 & $1.73 \pm 0.12$ & $1.09 \pm 0.18$ & $1.71 \pm 0.70$ & $-0.02 \pm 0.31$ & nan $\pm \inf$ \\
\hline 37.5 & $1.88 \pm 0.12$ & $1.30 \pm 0.15$ & $1.06 \pm 0.24$ & $0.92 \pm 0.54$ & $1.14 \pm 1.39$ \\
\hline 52.5 & $1.81 \pm 0.11$ & $1.35 \pm 0.11$ & $1.23 \pm 0.15$ & $0.86 \pm 0.21$ & $0.96 \pm 0.33$ \\
\hline 67.5 & $1.38 \pm 0.10$ & $1.32 \pm 0.10$ & $1.66 \pm 0.14$ & $1.50 \pm 0.17$ & $1.47 \pm 0.16$ \\
\hline 82.5 & $1.34 \pm 0.09$ & $1.30 \pm 0.09$ & $1.14 \pm 0.10$ & $0.81 \pm 0.09$ & $0.63 \pm 0.06$ \\
\hline 97.5 & $1.19 \pm 0.08$ & $0.90 \pm 0.08$ & $0.87 \pm 0.08$ & $0.64 \pm 0.07$ & $0.33 \pm 0.04$ \\
\hline 112.5 & $1.28 \pm 0.09$ & $0.91 \pm 0.08$ & $0.86 \pm 0.08$ & $0.58 \pm 0.07$ & $0.43 \pm 0.04$ \\
\hline 127.5 & $1.16 \pm 0.08$ & $0.93 \pm 0.08$ & $0.73 \pm 0.08$ & $0.55 \pm 0.07$ & $0.38 \pm 0.04$ \\
\hline 142.5 & $1.22 \pm 0.09$ & $0.80 \pm 0.07$ & $0.74 \pm 0.08$ & $0.46 \pm 0.06$ & $0.36 \pm 0.03$ \\
\hline 157.5 & $1.48 \pm 0.10$ & $0.85 \pm 0.08$ & $0.64 \pm 0.07$ & $0.54 \pm 0.06$ & $0.36 \pm 0.03$ \\
\hline 172.5 & $1.19 \pm 0.09$ & $0.82 \pm 0.08$ & $0.64 \pm 0.07$ & $0.53 \pm 0.07$ & $0.39 \pm 0.04$ \\
\hline 187.5 & $1.48 \pm 0.10$ & $0.86 \pm 0.08$ & $0.76 \pm 0.08$ & $0.64 \pm 0.08$ & $0.45 \pm 0.05$ \\
\hline 202.5 & $1.65 \pm 0.12$ & $0.92 \pm 0.09$ & $0.62 \pm 0.08$ & $0.64 \pm 0.08$ & $0.54 \pm 0.06$ \\
\hline 217.5 & $1.45 \pm 0.11$ & $1.15 \pm 0.10$ & $0.99 \pm 0.10$ & $0.74 \pm 0.09$ & $0.78 \pm 0.07$ \\
\hline 232.5 & $1.60 \pm 0.12$ & $1.26 \pm 0.11$ & $1.28 \pm 0.12$ & $0.82 \pm 0.11$ & $0.90 \pm 0.08$ \\
\hline 247.5 & $1.71 \pm 0.12$ & $1.55 \pm 0.13$ & $1.30 \pm 0.14$ & $1.34 \pm 0.16$ & $1.05 \pm 0.10$ \\
\hline 262.5 & $1.94 \pm 0.13$ & $1.49 \pm 0.13$ & $1.61 \pm 0.17$ & $1.78 \pm 0.22$ & $1.90 \pm 0.16$ \\
\hline 277.5 & $1.93 \pm 0.13$ & $1.44 \pm 0.12$ & $1.43 \pm 0.15$ & $1.15 \pm 0.14$ & $1.08 \pm 0.11$ \\
\hline 292.5 & $1.86 \pm 0.12$ & $1.16 \pm 0.09$ & $1.13 \pm 0.10$ & $0.72 \pm 0.09$ & $0.67 \pm 0.07$ \\
\hline 307.5 & $1.75 \pm 0.11$ & $1.32 \pm 0.10$ & $1.20 \pm 0.12$ & $0.77 \pm 0.12$ & $0.93 \pm 0.17$ \\
\hline 322.5 & $2.09 \pm 0.12$ & $1.31 \pm 0.12$ & $1.35 \pm 0.23$ & $0.84 \pm 0.35$ & $-0.08 \pm 1.65$ \\
\hline 337.5 & $1.88 \pm 0.12$ & $1.75 \pm 0.19$ & $0.78 \pm 0.45$ & $-0.01 \pm 0.16$ & nan $\pm \inf$ \\
\hline 352.5 & $1.97 \pm 0.13$ & $1.51 \pm 0.22$ & $0.51 \pm 0.76$ & nan \pm nan & nan \pm inf \\
\hline
\end{tabular}

TABLE 32: Unpolarized cross section for Kin 484 in pb/GeV $V^{4}$ 


\begin{tabular}{|c|c|c|c|c|c|}
\hline & \multicolumn{5}{|c|}{$\sigma$} \\
\hline$\phi(\operatorname{deg})$ & $\begin{array}{l}\left\langle Q^{2}\right\rangle=7.044 \\
\left\langle x_{B}\right\rangle=0.494 \\
\left\langle t^{\prime}\right\rangle=-0.043\end{array}$ & $\begin{array}{l}\left\langle Q^{2}\right\rangle=7.093 \\
\left\langle x_{B}\right\rangle=0.498 \\
\left\langle t^{\prime}\right\rangle=-0.127\end{array}$ & $\begin{array}{l}\left\langle Q^{2}\right\rangle=7.115 \\
\left\langle x_{B}\right\rangle=0.499 \\
\left\langle t^{\prime}\right\rangle=-0.212\end{array}$ & $\begin{array}{l}\left\langle Q^{2}\right\rangle=7.106 \\
\left\langle x_{B}\right\rangle=0.499 \\
\left\langle t^{\prime}\right\rangle=-0.297\end{array}$ & $\begin{array}{l}\left\langle Q^{2}\right\rangle=7.102 \\
\left\langle x_{B}\right\rangle=0.498 \\
\left\langle t^{\prime}\right\rangle=-0.457\end{array}$ \\
\hline 7.5 & $-0.07 \pm 0.12$ & $0.16 \pm 0.30$ & $1.34 \pm 0.94$ & nan $\pm \inf$ & nan \pm nan \\
\hline 22.5 & $0.11 \pm 0.12$ & $0.17 \pm 0.21$ & $-0.10 \pm 0.72$ & $-0.03 \pm 0.48$ & nan \pm nan \\
\hline 37.5 & $0.19 \pm 0.13$ & $0.27 \pm 0.18$ & $0.33 \pm 0.28$ & $0.57 \pm 0.70$ & $-1.39 \pm 1.44$ \\
\hline 52.5 & $0.04 \pm 0.13$ & $0.51 \pm 0.15$ & $0.69 \pm 0.19$ & $0.51 \pm 0.27$ & $0.28 \pm 0.37$ \\
\hline 67.5 & $0.24 \pm 0.12$ & $0.25 \pm 0.13$ & $0.55 \pm 0.18$ & $0.62 \pm 0.22$ & $0.52 \pm 0.18$ \\
\hline 82.5 & $0.19 \pm 0.12$ & $0.19 \pm 0.12$ & $0.22 \pm 0.12$ & $0.39 \pm 0.11$ & $0.24 \pm 0.07$ \\
\hline 97.5 & $0.07 \pm 0.11$ & $0.35 \pm 0.10$ & $0.18 \pm 0.10$ & $-0.07 \pm 0.08$ & $0.14 \pm 0.05$ \\
\hline 112.5 & $0.32 \pm 0.11$ & $0.19 \pm 0.10$ & $0.04 \pm 0.10$ & $0.03 \pm 0.07$ & $0.13 \pm 0.05$ \\
\hline 127.5 & $0.24 \pm 0.11$ & $0.22 \pm 0.10$ & $0.15 \pm 0.09$ & $0.10 \pm 0.07$ & $0.07 \pm 0.04$ \\
\hline 142.5 & $0.15 \pm 0.11$ & $0.08 \pm 0.09$ & $0.26 \pm 0.09$ & $-0.02 \pm 0.06$ & $0.04 \pm 0.04$ \\
\hline 157.5 & $0.17 \pm 0.13$ & $0.24 \pm 0.09$ & $0.20 \pm 0.08$ & $0.12 \pm 0.05$ & $0.05 \pm 0.04$ \\
\hline 172.5 & $0.16 \pm 0.13$ & $-0.04 \pm 0.09$ & $0.04 \pm 0.08$ & $-0.06 \pm 0.05$ & $0.07 \pm 0.04$ \\
\hline 187.5 & $0.13 \pm 0.13$ & $-0.14 \pm 0.10$ & $-0.06 \pm 0.09$ & $0.09 \pm 0.06$ & $-0.01 \pm 0.05$ \\
\hline 202.5 & $-0.01 \pm 0.15$ & $-0.16 \pm 0.11$ & $-0.00 \pm 0.09$ & $-0.04 \pm 0.07$ & $-0.13 \pm 0.06$ \\
\hline 217.5 & $-0.01 \pm 0.14$ & $-0.16 \pm 0.12$ & $-0.05 \pm 0.11$ & $0.05 \pm 0.09$ & $0.01 \pm 0.08$ \\
\hline 232.5 & $-0.26 \pm 0.16$ & $-0.06 \pm 0.14$ & $-0.20 \pm 0.14$ & $-0.10 \pm 0.11$ & $-0.15 \pm 0.09$ \\
\hline 247.5 & $-0.10 \pm 0.17$ & $-0.36 \pm 0.17$ & $-0.42 \pm 0.17$ & $-0.44 \pm 0.17$ & $-0.46 \pm 0.12$ \\
\hline 262.5 & $-0.03 \pm 0.17$ & $-0.61 \pm 0.17$ & $-0.69 \pm 0.21$ & $-0.46 \pm 0.24$ & $-0.40 \pm 0.18$ \\
\hline 277.5 & $-0.28 \pm 0.17$ & $-0.45 \pm 0.16$ & $-0.38 \pm 0.18$ & $-0.29 \pm 0.17$ & $-0.22 \pm 0.12$ \\
\hline 292.5 & $-0.15 \pm 0.15$ & $-0.39 \pm 0.12$ & $-0.32 \pm 0.13$ & $-0.17 \pm 0.11$ & $-0.12 \pm 0.08$ \\
\hline 307.5 & $-0.03 \pm 0.13$ & $-0.10 \pm 0.13$ & $-0.40 \pm 0.15$ & $-0.09 \pm 0.17$ & $-0.23 \pm 0.19$ \\
\hline 322.5 & $0.00 \pm 0.12$ & $-0.09 \pm 0.15$ & $0.01 \pm 0.27$ & $0.75 \pm 0.46$ & $-1.25 \pm 1.82$ \\
\hline 337.5 & $0.02 \pm 0.11$ & $-0.48 \pm 0.22$ & $0.33 \pm 0.48$ & $0.01 \pm 0.23$ & nan \pm nan \\
\hline 352.5 & $-0.02 \pm 0.12$ & $0.10 \pm 0.26$ & $1.07 \pm 0.71$ & nan \pm -inf & nan \pm nan \\
\hline
\end{tabular}

TABLE 33: Helicity-Dependent cross section for Kin 484 in pb/GeV $V^{4}$ 


\begin{tabular}{|c|c|c|c|c|c|}
\hline & \multicolumn{5}{|c|}{$\sigma$} \\
\hline$\phi(\operatorname{deg})$ & $\begin{array}{l}\left\langle Q^{2}\right\rangle=5.604 \\
\left\langle x_{B}\right\rangle=0.610 \\
\left\langle t^{\prime}\right\rangle=-0.068\end{array}$ & $\begin{array}{l}\left\langle Q^{2}\right\rangle=5.617 \\
\left\langle x_{B}\right\rangle=0.612 \\
\left\langle t^{\prime}\right\rangle=-0.200\end{array}$ & $\begin{array}{l}\left\langle Q^{2}\right\rangle=5.638 \\
\left\langle x_{B}\right\rangle=0.615 \\
\left\langle t^{\prime}\right\rangle=-0.333\end{array}$ & $\begin{array}{l}\left\langle Q^{2}\right\rangle=5.653 \\
\left\langle x_{B}\right\rangle=0.617 \\
\left\langle t^{\prime}\right\rangle=-0.467\end{array}$ & $\begin{array}{l}\left\langle Q^{2}\right\rangle=5.659 \\
\left\langle x_{B}\right\rangle=0.616 \\
\left\langle t^{\prime}\right\rangle=-0.654\end{array}$ \\
\hline 7.5 & $0.59 \pm 0.04$ & $0.42 \pm 0.04$ & $0.43 \pm 0.06$ & $0.30 \pm 0.11$ & $0.25 \pm 0.21$ \\
\hline 22.5 & $0.56 \pm 0.04$ & $0.40 \pm 0.04$ & $0.28 \pm 0.05$ & $0.32 \pm 0.08$ & $-0.06 \pm 0.13$ \\
\hline 37.5 & $0.62 \pm 0.04$ & $0.45 \pm 0.03$ & $0.36 \pm 0.04$ & $0.32 \pm 0.05$ & $0.14 \pm 0.05$ \\
\hline 52.5 & $0.56 \pm 0.03$ & $0.53 \pm 0.04$ & $0.31 \pm 0.03$ & $0.28 \pm 0.04$ & $0.21 \pm 0.04$ \\
\hline 67.5 & $0.58 \pm 0.04$ & $0.42 \pm 0.03$ & $0.34 \pm 0.03$ & $0.28 \pm 0.03$ & $0.27 \pm 0.03$ \\
\hline 82.5 & $0.57 \pm 0.04$ & $0.50 \pm 0.04$ & $0.34 \pm 0.03$ & $0.26 \pm 0.03$ & $0.24 \pm 0.03$ \\
\hline 97.5 & $0.53 \pm 0.03$ & $0.47 \pm 0.04$ & $0.31 \pm 0.03$ & $0.26 \pm 0.03$ & $0.20 \pm 0.03$ \\
\hline 112.5 & $0.53 \pm 0.04$ & $0.37 \pm 0.03$ & $0.29 \pm 0.03$ & $0.22 \pm 0.03$ & $0.23 \pm 0.03$ \\
\hline 127.5 & $0.47 \pm 0.03$ & $0.38 \pm 0.03$ & $0.31 \pm 0.03$ & $0.22 \pm 0.03$ & $0.20 \pm 0.02$ \\
\hline 142.5 & $0.47 \pm 0.03$ & $0.39 \pm 0.03$ & $0.34 \pm 0.03$ & $0.26 \pm 0.03$ & $0.26 \pm 0.03$ \\
\hline 157.5 & $0.45 \pm 0.03$ & $0.33 \pm 0.03$ & $0.33 \pm 0.03$ & $0.23 \pm 0.03$ & $0.26 \pm 0.03$ \\
\hline 172.5 & $0.44 \pm 0.03$ & $0.37 \pm 0.03$ & $0.31 \pm 0.03$ & $0.34 \pm 0.04$ & $0.30 \pm 0.03$ \\
\hline 187.5 & $0.48 \pm 0.03$ & $0.34 \pm 0.03$ & $0.37 \pm 0.04$ & $0.36 \pm 0.04$ & $0.34 \pm 0.03$ \\
\hline 202.5 & $0.47 \pm 0.03$ & $0.39 \pm 0.03$ & $0.37 \pm 0.04$ & $0.28 \pm 0.04$ & $0.31 \pm 0.03$ \\
\hline 217.5 & $0.49 \pm 0.03$ & $0.44 \pm 0.04$ & $0.36 \pm 0.04$ & $0.34 \pm 0.04$ & $0.31 \pm 0.03$ \\
\hline 232.5 & $0.51 \pm 0.04$ & $0.47 \pm 0.04$ & $0.46 \pm 0.04$ & $0.34 \pm 0.04$ & $0.33 \pm 0.03$ \\
\hline 247.5 & $0.55 \pm 0.04$ & $0.47 \pm 0.04$ & $0.38 \pm 0.04$ & $0.34 \pm 0.04$ & $0.32 \pm 0.04$ \\
\hline 262.5 & $0.54 \pm 0.04$ & $0.39 \pm 0.04$ & $0.45 \pm 0.04$ & $0.27 \pm 0.04$ & $0.20 \pm 0.03$ \\
\hline 277.5 & $0.52 \pm 0.04$ & $0.51 \pm 0.04$ & $0.40 \pm 0.04$ & $0.28 \pm 0.04$ & $0.29 \pm 0.03$ \\
\hline 292.5 & $0.55 \pm 0.04$ & $0.57 \pm 0.04$ & $0.37 \pm 0.03$ & $0.32 \pm 0.04$ & $0.29 \pm 0.03$ \\
\hline 307.5 & $0.63 \pm 0.04$ & $0.52 \pm 0.04$ & $0.35 \pm 0.03$ & $0.34 \pm 0.04$ & $0.29 \pm 0.04$ \\
\hline 322.5 & $0.63 \pm 0.04$ & $0.56 \pm 0.04$ & $0.33 \pm 0.04$ & $0.35 \pm 0.05$ & $0.19 \pm 0.06$ \\
\hline 337.5 & $0.63 \pm 0.04$ & $0.56 \pm 0.04$ & $0.46 \pm 0.05$ & $0.35 \pm 0.08$ & $0.48 \pm 0.14$ \\
\hline 352.5 & $0.66 \pm 0.04$ & $0.52 \pm 0.04$ & $0.42 \pm 0.06$ & $0.22 \pm 0.13$ & $0.10 \pm 0.28$ \\
\hline
\end{tabular}

TABLE 34: Unpolarized cross section for Kin $601 \mathrm{in} \mathrm{pb/GeV}$ 


\begin{tabular}{|c|c|c|c|c|c|}
\hline & \multicolumn{5}{|c|}{$\sigma$} \\
\hline$\phi(\operatorname{deg})$ & $\begin{array}{l}\left\langle Q^{2}\right\rangle=5.604 \\
\left\langle x_{B}\right\rangle=0.610 \\
\left\langle t^{\prime}\right\rangle=-0.068\end{array}$ & $\begin{array}{l}\left\langle Q^{2}\right\rangle=5.617 \\
\left\langle x_{B}\right\rangle=0.612 \\
\left\langle t^{\prime}\right\rangle=-0.200\end{array}$ & $\begin{array}{l}\left\langle Q^{2}\right\rangle=5.638 \\
\left\langle x_{B}\right\rangle=0.615 \\
\left\langle t^{\prime}\right\rangle=-0.333\end{array}$ & $\begin{array}{l}\left\langle Q^{2}\right\rangle=5.653 \\
\left\langle x_{B}\right\rangle=0.617 \\
\left\langle t^{\prime}\right\rangle=-0.467\end{array}$ & $\begin{array}{l}\left\langle Q^{2}\right\rangle=5.659 \\
\left\langle x_{B}\right\rangle=0.616 \\
\left\langle t^{\prime}\right\rangle=-0.654\end{array}$ \\
\hline 7.5 & $-0.01 \pm 0.03$ & $0.04 \pm 0.04$ & $0.02 \pm 0.05$ & $-0.01 \pm 0.10$ & $-0.20 \pm 0.15$ \\
\hline 22.5 & $0.03 \pm 0.04$ & $0.07 \pm 0.04$ & $0.09 \pm 0.04$ & $0.17 \pm 0.08$ & $0.18 \pm 0.11$ \\
\hline 37.5 & $0.07 \pm 0.05$ & $0.09 \pm 0.04$ & $0.09 \pm 0.04$ & $0.10 \pm 0.06$ & $0.06 \pm 0.05$ \\
\hline 52.5 & $0.19 \pm 0.04$ & $0.15 \pm 0.04$ & $0.03 \pm 0.04$ & $0.10 \pm 0.04$ & $0.11 \pm 0.04$ \\
\hline 67.5 & $0.12 \pm 0.04$ & $0.10 \pm 0.04$ & $0.08 \pm 0.04$ & $0.03 \pm 0.04$ & $0.02 \pm 0.03$ \\
\hline 82.5 & $0.05 \pm 0.04$ & $0.07 \pm 0.04$ & $0.11 \pm 0.04$ & $-0.00 \pm 0.04$ & $0.01 \pm 0.03$ \\
\hline 97.5 & $0.09 \pm 0.04$ & $0.10 \pm 0.04$ & $0.05 \pm 0.04$ & $0.04 \pm 0.04$ & $0.08 \pm 0.03$ \\
\hline 112.5 & $0.05 \pm 0.04$ & $0.05 \pm 0.04$ & $0.07 \pm 0.04$ & $0.00 \pm 0.03$ & $0.04 \pm 0.03$ \\
\hline 127.5 & $0.06 \pm 0.04$ & $0.06 \pm 0.03$ & $0.07 \pm 0.04$ & $0.05 \pm 0.03$ & $0.04 \pm 0.02$ \\
\hline 142.5 & $0.06 \pm 0.04$ & $0.02 \pm 0.03$ & $0.04 \pm 0.04$ & $0.07 \pm 0.03$ & $-0.04 \pm 0.03$ \\
\hline 157.5 & $0.05 \pm 0.04$ & $-0.01 \pm 0.03$ & $-0.02 \pm 0.04$ & $0.03 \pm 0.03$ & $0.01 \pm 0.03$ \\
\hline 172.5 & $0.00 \pm 0.03$ & $-0.02 \pm 0.03$ & $0.00 \pm 0.03$ & $0.04 \pm 0.03$ & $-0.02 \pm 0.03$ \\
\hline 187.5 & $0.04 \pm 0.07$ & $-0.03 \pm 0.05$ & $-0.02 \pm 0.05$ & $-0.03 \pm 0.04$ & $-0.02 \pm 0.04$ \\
\hline 202.5 & $-0.03 \pm 0.05$ & $-0.02 \pm 0.04$ & $0.01 \pm 0.04$ & $0.00 \pm 0.03$ & $-0.06 \pm 0.03$ \\
\hline 217.5 & $-0.03 \pm 0.05$ & $-0.07 \pm 0.04$ & $0.00 \pm 0.04$ & $-0.02 \pm 0.03$ & $-0.03 \pm 0.03$ \\
\hline 232.5 & $-0.08 \pm 0.05$ & $-0.03 \pm 0.04$ & $-0.08 \pm 0.05$ & $0.02 \pm 0.04$ & $-0.08 \pm 0.04$ \\
\hline 247.5 & $-0.06 \pm 0.05$ & $-0.08 \pm 0.04$ & $-0.13 \pm 0.04$ & $-0.06 \pm 0.04$ & $-0.04 \pm 0.04$ \\
\hline 262.5 & $-0.04 \pm 0.05$ & $-0.12 \pm 0.04$ & $-0.13 \pm 0.05$ & $-0.07 \pm 0.04$ & $-0.16 \pm 0.04$ \\
\hline 277.5 & $-0.13 \pm 0.05$ & $-0.10 \pm 0.04$ & $-0.10 \pm 0.04$ & $-0.08 \pm 0.04$ & $-0.03 \pm 0.04$ \\
\hline 292.5 & $-0.18 \pm 0.05$ & $-0.08 \pm 0.04$ & $-0.10 \pm 0.04$ & $-0.04 \pm 0.04$ & $-0.02 \pm 0.04$ \\
\hline 307.5 & $-0.10 \pm 0.05$ & $-0.03 \pm 0.04$ & $-0.09 \pm 0.04$ & $-0.17 \pm 0.05$ & $-0.09 \pm 0.04$ \\
\hline 322.5 & $-0.09 \pm 0.05$ & $-0.05 \pm 0.04$ & $-0.06 \pm 0.05$ & $-0.08 \pm 0.06$ & $0.01 \pm 0.06$ \\
\hline 337.5 & $-0.08 \pm 0.05$ & $-0.08 \pm 0.05$ & $0.01 \pm 0.06$ & $0.01 \pm 0.09$ & $0.03 \pm 0.12$ \\
\hline 352.5 & $-0.12 \pm 0.08$ & $-0.12 \pm 0.06$ & $0.00 \pm 0.07$ & $-0.06 \pm 0.14$ & $-0.04 \pm 0.24$ \\
\hline
\end{tabular}

TABLE 35: Helicity-Dependent cross section for Kin 601 in pb/GeV ${ }^{4}$ 


\begin{tabular}{|c|c|c|c|c|c|}
\hline & \multicolumn{5}{|c|}{$\sigma$} \\
\hline$\phi(\operatorname{deg})$ & $\begin{array}{l}\left\langle Q^{2}\right\rangle=8.440 \\
\left\langle x_{B}\right\rangle=0.608 \\
\left\langle t^{\prime}\right\rangle=-0.060\end{array}$ & $\begin{array}{l}\left\langle Q^{2}\right\rangle=8.453 \\
\left\langle x_{B}\right\rangle=0.609 \\
\left\langle t^{\prime}\right\rangle=-0.177\end{array}$ & $\begin{array}{l}\left\langle Q^{2}\right\rangle=8.481 \\
\left\langle x_{B}\right\rangle=0.611 \\
\left\langle t^{\prime}\right\rangle=-0.294\end{array}$ & $\begin{array}{l}\left\langle Q^{2}\right\rangle=8.501 \\
\left\langle x_{B}\right\rangle=0.613 \\
\left\langle t^{\prime}\right\rangle=-0.412\end{array}$ & $\begin{array}{l}\left\langle Q^{2}\right\rangle=8.513 \\
\left\langle x_{B}\right\rangle=0.613 \\
\left\langle t^{\prime}\right\rangle=-0.577\end{array}$ \\
\hline 7.5 & $0.33 \pm 0.02$ & $0.28 \pm 0.02$ & $0.26 \pm 0.03$ & $0.23 \pm 0.07$ & $0.16 \pm 0.16$ \\
\hline 22.5 & $0.34 \pm 0.02$ & $0.28 \pm 0.02$ & $0.20 \pm 0.03$ & $0.20 \pm 0.04$ & $0.19 \pm 0.07$ \\
\hline 37.5 & $0.32 \pm 0.02$ & $0.25 \pm 0.02$ & $0.17 \pm 0.02$ & $0.12 \pm 0.02$ & $0.11 \pm 0.03$ \\
\hline 52.5 & $0.30 \pm 0.02$ & $0.25 \pm 0.02$ & $0.19 \pm 0.02$ & $0.12 \pm 0.02$ & $0.11 \pm 0.02$ \\
\hline 67.5 & $0.29 \pm 0.02$ & $0.26 \pm 0.02$ & $0.19 \pm 0.02$ & $0.11 \pm 0.01$ & $0.11 \pm 0.01$ \\
\hline 82.5 & $0.30 \pm 0.02$ & $0.22 \pm 0.02$ & $0.17 \pm 0.01$ & $0.11 \pm 0.01$ & $0.11 \pm 0.01$ \\
\hline 97.5 & $0.28 \pm 0.02$ & $0.23 \pm 0.02$ & $0.14 \pm 0.01$ & $0.12 \pm 0.01$ & $0.08 \pm 0.01$ \\
\hline 112.5 & $0.25 \pm 0.02$ & $0.18 \pm 0.01$ & $0.15 \pm 0.01$ & $0.11 \pm 0.01$ & $0.08 \pm 0.01$ \\
\hline 127.5 & $0.24 \pm 0.02$ & $0.16 \pm 0.01$ & $0.12 \pm 0.01$ & $0.09 \pm 0.01$ & $0.06 \pm 0.01$ \\
\hline 142.5 & $0.24 \pm 0.02$ & $0.16 \pm 0.01$ & $0.13 \pm 0.01$ & $0.09 \pm 0.01$ & $0.05 \pm 0.01$ \\
\hline 157.5 & $0.23 \pm 0.02$ & $0.17 \pm 0.01$ & $0.11 \pm 0.01$ & $0.08 \pm 0.01$ & $0.07 \pm 0.01$ \\
\hline 172.5 & $0.23 \pm 0.01$ & $0.15 \pm 0.01$ & $0.13 \pm 0.01$ & $0.08 \pm 0.01$ & $0.06 \pm 0.01$ \\
\hline 187.5 & $0.21 \pm 0.01$ & $0.14 \pm 0.01$ & $0.13 \pm 0.01$ & $0.09 \pm 0.01$ & $0.06 \pm 0.01$ \\
\hline 202.5 & $0.22 \pm 0.02$ & $0.18 \pm 0.01$ & $0.13 \pm 0.01$ & $0.10 \pm 0.01$ & $0.07 \pm 0.01$ \\
\hline 217.5 & $0.24 \pm 0.02$ & $0.18 \pm 0.01$ & $0.14 \pm 0.01$ & $0.10 \pm 0.01$ & $0.08 \pm 0.01$ \\
\hline 232.5 & $0.24 \pm 0.02$ & $0.20 \pm 0.02$ & $0.16 \pm 0.01$ & $0.13 \pm 0.01$ & $0.08 \pm 0.01$ \\
\hline 247.5 & $0.27 \pm 0.02$ & $0.24 \pm 0.02$ & $0.17 \pm 0.02$ & $0.14 \pm 0.01$ & $0.11 \pm 0.01$ \\
\hline 262.5 & $0.27 \pm 0.02$ & $0.26 \pm 0.02$ & $0.18 \pm 0.02$ & $0.12 \pm 0.02$ & $0.11 \pm 0.01$ \\
\hline 277.5 & $0.31 \pm 0.02$ & $0.22 \pm 0.02$ & $0.23 \pm 0.02$ & $0.15 \pm 0.02$ & $0.13 \pm 0.01$ \\
\hline 292.5 & $0.31 \pm 0.02$ & $0.27 \pm 0.02$ & $0.25 \pm 0.02$ & $0.18 \pm 0.02$ & $0.16 \pm 0.02$ \\
\hline 307.5 & $0.32 \pm 0.02$ & $0.27 \pm 0.02$ & $0.24 \pm 0.02$ & $0.18 \pm 0.02$ & $0.16 \pm 0.02$ \\
\hline 322.5 & $0.31 \pm 0.02$ & $0.27 \pm 0.02$ & $0.24 \pm 0.02$ & $0.18 \pm 0.03$ & $0.17 \pm 0.04$ \\
\hline 337.5 & $0.35 \pm 0.02$ & $0.29 \pm 0.02$ & $0.21 \pm 0.02$ & $0.26 \pm 0.05$ & $0.09 \pm 0.07$ \\
\hline 352.5 & $0.34 \pm 0.02$ & $0.30 \pm 0.02$ & $0.21 \pm 0.03$ & $0.15 \pm 0.07$ & $0.44 \pm 0.18$ \\
\hline
\end{tabular}

TABLE 36: Unpolarized cross section for Kin 603 in pb/GeV 


\begin{tabular}{|c|c|c|c|c|c|}
\hline & \multicolumn{5}{|c|}{$\sigma$} \\
\hline$\phi(\operatorname{deg})$ & $\begin{array}{l}\left\langle Q^{2}\right\rangle=8.440 \\
\left\langle x_{B}\right\rangle=0.608 \\
\left\langle t^{\prime}\right\rangle=-0.060\end{array}$ & $\begin{array}{l}\left\langle Q^{2}\right\rangle=8.453 \\
\left\langle x_{B}\right\rangle=0.609 \\
\left\langle t^{\prime}\right\rangle=-0.177\end{array}$ & $\begin{array}{l}\left\langle Q^{2}\right\rangle=8.481 \\
\left\langle x_{B}\right\rangle=0.611 \\
\left\langle t^{\prime}\right\rangle=-0.294\end{array}$ & $\begin{array}{l}\left\langle Q^{2}\right\rangle=8.501 \\
\left\langle x_{B}\right\rangle=0.613 \\
\left\langle t^{\prime}\right\rangle=-0.412\end{array}$ & $\begin{array}{l}\left\langle Q^{2}\right\rangle=8.513 \\
\left\langle x_{B}\right\rangle=0.613 \\
\left\langle t^{\prime}\right\rangle=-0.577\end{array}$ \\
\hline 7.5 & $0.00 \pm 0.02$ & $0.01 \pm 0.03$ & $-0.00 \pm 0.03$ & $-0.01 \pm 0.05$ & $0.04 \pm 0.09$ \\
\hline 22.5 & $-0.00 \pm 0.02$ & $0.06 \pm 0.02$ & $-0.00 \pm 0.03$ & $0.05 \pm 0.04$ & $0.05 \pm 0.06$ \\
\hline 37.5 & $-0.00 \pm 0.02$ & $0.04 \pm 0.02$ & $0.00 \pm 0.02$ & $0.05 \pm 0.03$ & $0.03 \pm 0.03$ \\
\hline 52.5 & $0.02 \pm 0.02$ & $0.05 \pm 0.02$ & $0.05 \pm 0.02$ & $0.04 \pm 0.02$ & $0.03 \pm 0.02$ \\
\hline 67.5 & $0.04 \pm 0.02$ & $0.05 \pm 0.02$ & $0.05 \pm 0.02$ & $0.05 \pm 0.02$ & $0.03 \pm 0.01$ \\
\hline 82.5 & $0.03 \pm 0.02$ & $0.05 \pm 0.02$ & $0.03 \pm 0.02$ & $0.04 \pm 0.02$ & $0.01 \pm 0.01$ \\
\hline 97.5 & $0.06 \pm 0.02$ & $0.03 \pm 0.02$ & $0.03 \pm 0.02$ & $0.03 \pm 0.01$ & $0.01 \pm 0.01$ \\
\hline 112.5 & $0.05 \pm 0.02$ & $0.03 \pm 0.02$ & $0.04 \pm 0.02$ & $0.03 \pm 0.01$ & $0.02 \pm 0.01$ \\
\hline 127.5 & $0.02 \pm 0.02$ & $0.02 \pm 0.01$ & $0.03 \pm 0.01$ & $0.04 \pm 0.01$ & $0.01 \pm 0.01$ \\
\hline 142.5 & $-0.00 \pm 0.02$ & $-0.00 \pm 0.01$ & $0.03 \pm 0.01$ & $-0.00 \pm 0.01$ & $0.01 \pm 0.01$ \\
\hline 157.5 & $0.01 \pm 0.02$ & $0.00 \pm 0.01$ & $0.02 \pm 0.01$ & $0.01 \pm 0.01$ & $0.00 \pm 0.01$ \\
\hline 172.5 & $0.02 \pm 0.02$ & $0.01 \pm 0.01$ & $-0.01 \pm 0.01$ & $-0.02 \pm 0.01$ & $0.03 \pm 0.01$ \\
\hline 187.5 & $-0.01 \pm 0.02$ & $-0.00 \pm 0.01$ & $0.00 \pm 0.02$ & $-0.01 \pm 0.01$ & $0.00 \pm 0.01$ \\
\hline 202.5 & $-0.01 \pm 0.02$ & $-0.00 \pm 0.01$ & $-0.02 \pm 0.02$ & $0.00 \pm 0.01$ & $-0.01 \pm 0.01$ \\
\hline 217.5 & $-0.02 \pm 0.02$ & $-0.03 \pm 0.02$ & $-0.01 \pm 0.02$ & $-0.01 \pm 0.01$ & $-0.01 \pm 0.01$ \\
\hline 232.5 & $-0.02 \pm 0.02$ & $-0.01 \pm 0.02$ & $-0.05 \pm 0.02$ & $0.01 \pm 0.01$ & $-0.02 \pm 0.01$ \\
\hline 247.5 & $-0.05 \pm 0.02$ & $-0.05 \pm 0.02$ & $-0.04 \pm 0.02$ & $-0.04 \pm 0.02$ & $-0.05 \pm 0.01$ \\
\hline 262.5 & $-0.03 \pm 0.02$ & $-0.02 \pm 0.02$ & $-0.07 \pm 0.02$ & $0.02 \pm 0.02$ & $-0.02 \pm 0.02$ \\
\hline 277.5 & $-0.03 \pm 0.02$ & $-0.07 \pm 0.02$ & $-0.08 \pm 0.02$ & $-0.02 \pm 0.02$ & $-0.03 \pm 0.02$ \\
\hline 292.5 & $-0.01 \pm 0.02$ & $-0.03 \pm 0.02$ & $-0.11 \pm 0.02$ & $-0.02 \pm 0.02$ & $-0.02 \pm 0.02$ \\
\hline 307.5 & $-0.04 \pm 0.02$ & $-0.04 \pm 0.02$ & $-0.06 \pm 0.02$ & $-0.06 \pm 0.03$ & $0.02 \pm 0.02$ \\
\hline 322.5 & $-0.01 \pm 0.02$ & $-0.06 \pm 0.02$ & $-0.05 \pm 0.02$ & $-0.05 \pm 0.03$ & $-0.07 \pm 0.03$ \\
\hline 337.5 & $-0.03 \pm 0.02$ & $-0.04 \pm 0.03$ & $-0.03 \pm 0.03$ & $-0.07 \pm 0.04$ & $0.05 \pm 0.05$ \\
\hline 352.5 & $0.00 \pm 0.02$ & $0.00 \pm 0.03$ & $-0.02 \pm 0.03$ & $-0.05 \pm 0.06$ & $-0.05 \pm 0.11$ \\
\hline
\end{tabular}

TABLE 37: Helicity-Dependent cross section for Kin 603 in pb/GeV ${ }^{4}$ 


\section{BIBLIOGRAPHY}

[1] F.D. Aaron et al. Deeply Virtual Compton Scattering and its Beam Charge Asymmetry in e+- Collisions at HERA. Phys. Lett. B, 681:391-399, 2009. arXiv:0907.5289, doi:10.1016/j.physletb.2009.10.035.

[2] A. Accardi et al. Electron ion collider: The next qcd frontier - understanding the glue that binds us all, 2014. arXiv:1212.1701.

[3] D. Adams et al. A new measurement of the spin-dependent structure function g1(x) of the deuteron. Physics Letters B, 357(1):248 - 254, 1995. URL: http: //www.sciencedirect.com/science/article/pii/037026939500898U, doi:https: //doi.org/10.1016/0370-2693(95)00898-U.

[4] C. Adloff et al. Measurement of deeply virtual Compton scattering at HERA. Phys. Lett. B, 517:47-58, 2001. arXiv:hep-ex/0107005, doi:10.1016/S0370-2693(01)00939-X.

[5] J. Alcorn et al. Basic Instrumentation for Hall A at Jefferson Lab. Nucl. Instrum. Meth., A522:294-346, 2004. doi:10.1016/j.nima.2003.11.415.

[6] K. Allada et al. PMT signal increase using a wavelength shifting paint. Nucl. Instrum. Meth, 782:87 - 91, 2015. URL: http://www.sciencedirect.com/science/article/ pii/S0168900215001898, doi:https://doi.org/10.1016/j.nima.2015.02.012.

[7] G. Altarelli and G. Parisi. Asymptotic freedom in parton language. Nuclear Physics B, 126(2):298 - 318, 1977. URL: http://www. sciencedirect.com/science/article/ pii/0550321377903844, doi:https://doi.org/10.1016/0550-3213(77)90384-4.

[8] J. Arrington. Introduction to SIMC. Hall A Technical Note., 2000. URL: https: //hallaweb.jlab.org/wiki/images/0/07/Simc.pdf.

[9] J. Arrington, W. Melnitchouk, and J. A. Tjon. Global analysis of proton elastic form factor data with two-photon exchange corrections. Physical Review C, 76(3), Sep 2007. URL: http://dx.doi.org/10.1103/PhysRevC.76.035205, doi:10.1103/physrevc. 76.035205,

[10] Alessandro Bacchetta, Umberto D'Alesio, Markus Diehl, and C. Miller. Single-spin asymmetries: The trento conventions. Physical Review D, 70(11), Dec 2004. URL: http: //dx.doi.org/10.1103/PhysRevD.70.117504, doi:10.1103/physrevd.70.117504. 
[11] Henri Becquerel. Nobel lecture. URL: www.nobelprize.org/prizes/physics/1903/ becquerel/lecture/.

[12] A. V. Belitsky and D. Müller. Exclusive electroproduction revisited: Treating kinematical effects. Phys. Rev. D, 82:074010, Oct 2010. URL: https://link.aps.org/doi/ 10.1103/PhysRevD.82.074010, doi:10.1103/PhysRevD.82.074010.

[13] A.V. Belitsky, D. Müller, and Y. Ji. Compton scattering: From deeply virtual to quasireal. Nuclear Physics B, 878:214 - 268, 2014. URL: http://www.sciencedirect. com/science/article/pii/S0550321313005786, doi:https://doi.org/10.1016/j. nuclphysb.2013.11.014.

[14] A.V. Belitsky, D. Müller, and A. Kirchner. Theory of deeply virtual Compton scattering on the nucleon. Nuclear Physics B, 629(1):323 - 392, 2002. URL: http: //www.sciencedirect.com/science/article/pii/S055032130200144X, doi:https: //doi.org/10.1016/S0550-3213(02)00144-X.

[15] A.V. Belitsky and A.V. Radyushkin. Unraveling hadron structure with generalized parton distributions. Physics Reports, 418(1):1 - 387, 2005. URL: http: //www.sciencedirect.com/science/article/pii/S0370157305002644, doi:https: //doi.org/10.1016/j.physrep.2005.06.002.

[16] Bishnu Karki. "Deep Exclusive $\pi^{0}$ Electroproduction Measured in Hall A at Jefferson Lab with the Upgraded CEBAF". PhD thesis, College of Arts and Science of Ohio University, 2020.

[17] J. D. Bjorken. Asymptotic sum rules at infinite momentum. Phys. Rev., 179:1547-1553, Mar 1969. URL: https://link.aps.org/doi/10.1103/PhysRev.179.1547, doi:10. 1103/PhysRev.179.1547.

[18] C. Muñoz Camacho. Diffusion Compton profondêment virtuelle dans le Hall A au Jefferson Laboratory. PhD thesis, de l'Universitẽ Paris VI, 2005.

[19] C. Muñoz Camacho et al. Scaling tests of the cross-section for deeply virtual compton scattering. Phys. Rev. Lett., 97:262002, 2006. arXiv:nucl-ex/0607029, doi:10.1103/ PhysRevLett.97.262002.

[20] G.F. Chew. *bootstrap* - a scientific idea? Science, 161:762-765, 1968. doi:10.1126/ science.161.3843.762. 
[21] M. E. Christy and P. E. Bosted. Empirical fit to precision inclusive electronproton cross sections in the resonance region. Phys. Rev. C, 81:055213, May 2010. URL: https://link.aps.org/doi/10.1103/PhysRevC.81.055213, doi:10. 1103/PhysRevC.81.055213.

[22] HERMES collaboration, A Airapetian, N Akopov, Z Akopov, A Andrus, E.C Aschenauer, W Augustyniak, R Avakian, A Avetissian, E Avetisyan, and et al. Measurement of azimuthal asymmetries with respect to both beam charge and transverse target polarization in exclusive electroproduction of real photons. Journal of High Energy Physics, 2008(06):066-066, Jun 2008. URL: http://dx.doi.org/10.1088/1126-6708/ 2008/06/066, doi:10.1088/1126-6708/2008/06/066.

[23] ZEUS collaboration. A measurement of theq2, wandtdependences of deeply virtual compton scattering at hera. Journal of High Energy Physics, 2009(05):108-108, May 2009. URL: http://dx.doi.org/10.1088/1126-6708/2009/05/108, doi:10.1088/ 1126-6708/2009/05/108.

[24] J. C. Cornejo. Compton Update for DVCS. URL: https://www.jlab.org/indico/ event/253/contribution/3/material/slides/0.pdf.

[25] D. Lhuillier. Diffusion Compton Virtuelle à basse énergie. PhD thesis, Université de Caen, 1997.

[26] M. Defurne et al. A glimpse of gluons through deeply virtual compton scattering on the proton. Nature Commun., 8(1):1408, 2017. arXiv:1703.09442, doi:10.1038/ s41467-017-01819-3.

[27] Nicole d'Hose, Silvia Niccolai, and Armine Rostomyan. Experimental overview of deeply virtual compton scattering. The European Physical Journal A, 52(6):151, 2016. doi: 10.1140/epja/i2016-16151-9.

[28] Mongi Dlamini. Measurement of Hard Exclusive Electroproduction of $\pi^{0}$ Meson Cross Section in Hall A of JLab with CEBAF at 12 GeV. PhD thesis, College of Arts and Science of Ohio University, 2018.

[29] Dr. Paul King. private communication.

[30] F. Feinstein. The analogue ring sampler: A front-end chip for ANTARES. Nuclear Instruments and Methods in Physics Research Section A: Accelerators, Spectrometers, 
Detectors and Associated Equipment, 504(1):258 - 261, 2003. Proceedings of the 3rd International Conference on New Developments in Photodetection. URL: http://www . sciencedirect.com/science/article/pii/S0168900203007733, doi:https://doi. org/10.1016/S0168-9002(03)00773-3.

[31] Richard P. Feynman. Very high-energy collisions of hadrons. Phys. Rev. Lett., 23:14151417, Dec 1969. URL: https://link.aps.org/doi/10.1103/PhysRevLett.23.1415, doi:10.1103/PhysRevLett.23.1415.

[32] K. G. Fissum et al. Vertical drift chambers for the Hall A high-resolution spectrometers at Jefferson Lab. Nucl. Instrum. Meth., A474:108-131, 2001. doi:10.1016/ S0168-9002(01)00875-0.

[33] Frédéric Georges. Deeply Virtual Compton Scattering at Jefferson Lab. PhD thesis, de l'Universitẽ Paris-Saclay, 2018.

[34] Arne Freyberger. Commissioning and Operation of $12 \mathrm{GeV}$ CEBAF. In 6th International Particle Accelerator Conference, page MOXGB2, 2015. doi:10.18429/ JACoW-IPAC2015-MOXGB2.

[35] J. J. Thomson M.A. F.R.S. Xl. cathode rays. The London, Edinburgh, and Dublin Philosophical Magazine and Journal of Science, 44(269):293-316, 1897. doi:10.1080/ 14786449708621070.

[36] M. Gell-Mann. A schematic model of baryons and mesons. Physics Letters, 8(3):214 - 215, 1964. URL: http://www.sciencedirect.com/science/article/pii/ S0031916364920013, doi:https://doi.org/10.1016/S0031-9163(64)92001-3.

[37] S. Glamazdin and R. Pomatsalyuk. Moller Polarimetry for DVCS, 2017. URL: https://www.jlab.org/indico/event/197/session/3/contribution/11/ material/slides/0.pdf.

[38] M. Guidal. Deep virtual compton scattering and the nucleon generalized parton distributions. Nuclear Physics A, 751:180-191, Apr 2005. URL: http://dx.doi.org/10. 1016/j.nuclphysa.2005.02.011, doi:10.1016/j.nuclphysa.2005.02.011.

[39] Gulakhshan Hamad. R-fuctions. URL: https://hallaweb.jlab.org/dvcslog/12+ $\mathrm{GeV} / 455$. 
[40] Ole Hansen. HRS Tracking. In Hall A DVCS Collaboration Meetings, Old Dominion University, December 2013. URL: https://www.jlab.org/indico/event/58/.

[41] Harps for Dummies, JLab page. URL: https://www.jlab.org/accel/beam_diag/ harp/harp_dummy.html.

[42] Douglas W. Higinbotham. Determination of the Beam Energy. In Hall A DVCS Collaboration Meetings, JLab, January 2017. URL: https://www.jlab.org/indico/event/ $197 /$.

[43] Robert Hofstadter. Electron Scattering and Nuclear Structure. Rev. Mod. Phys., 28:214254, Jul 1956. URL: https://link.aps.org/doi/10.1103/RevModPhys.28.214, doi: 10.1103/RevModPhys.28.214.

[44] Charles E. Hyde. Comment on Radiative Corrections in Virtual Compton Scattering. URL: https://hallaweb.jlab.org/dvcslog/DVCS2/131219_143249/Rad_Corr_VCS. pdf.

[45] Charles E. Hyde. E12-06-114 Radiative Corrections Update. URL: https: //hallaweb.jlab.org/dvcslog/12+GeV/200526_085453/RadiativeCorrections_ 26May2020.pdf.

[46] M. Iodice et al. The $\mathrm{CO}_{2}$ gas Cherenkov detectors for the Jefferson Lab Hall-A spectrometers. Nucl. Instrum. Meth., A411:223 - 237, 1998. URL: http://www. sciencedirect.com/science/article/pii/S0168900298002356, doi:https://doi. org/10.1016/S0168-9002(98)00235-6.

[47] Xiangdong Ji. Deeply virtual compton scattering. Physical Review D, 55(11):7114-7125, Jun 1997. URL: http://dx.doi.org/10.1103/PhysRevD.55.7114, doi:10.1103/ physrevd.55.7114.

[48] Xiangdong Ji. Gauge-invariant decomposition of nucleon spin. Phys. Rev. Lett., 78:610613, Jan 1997. URL: https://link.aps.org/doi/10.1103/PhysRevLett.78.610, doi:10.1103/PhysRevLett.78.610.

[49] R.T. Jones, M. Kornicer, A.R. Dzierba, J.L. Gunter, R. Lindenbusch, E. Scott, P. Smith, C. Steffen, S. Teige, P. Rubin, and E.S. Smith. A bootstrap method 
for gain calibration and resolution determination of a lead-glass calorimeter. $\mathrm{Nu}$ clear Instruments and Methods in Physics Research Section A: Accelerators, Spectrometers, Detectors and Associated Equipment, 566(2):366 - 374, 2006. URL: http: //www.sciencedirect.com/science/article/pii/S0168900206013556, doi:https: //doi.org/10.1016/j.nima.2006.07.061.

[50] B. Karki. Charge computation. URL: https://hallaweb.jlab.org/dvcslog/12+GeV/ 453 .

[51] B. Karki. DIS Cross section Update. URL: https://hallaweb.jlab.org/dvcslog/ 12+GeV/190429_154235/finalDISResult.pdf.

[52] B. Karki and J.Roche. BCMs' Calibration and Charge Normalization. URL: https: //hallaweb.jlab.org/dvcslog/12+GeV/349.

[53] Bishnu Karki. Private Communication.

[54] Henry W Kendall. Nobel lecture. URL: https://www.nobelprize.org/prizes/ physics/1990/kendall/lecture/.

[55] Jan Kretzschmar. Proton Structure Measurements and the HERAPDF fit. In 24th Lake Louise Winter Institute: Fundamental Interactions, 6 2009. arXiv:0906.1108.

[56] Krešimir Kumerički, Simonetta Liuti, and Hervé Moutarde. GPD phenomenology and DVCS fitting. The European Physical Journal A, 52(6), Jun 2016. URL: http://dx. doi.org/10.1140/epja/i2016-16157-3, doi:10.1140/epja/i2016-16157-3.

[57] Krešimir Kumerički and Dieter Müller. Deeply virtual Compton scattering at small $x$ and the access to the GPD H. Nuclear Physics B, 841(1-2):1-58, Dec 2010. URL: http://dx.doi.org/10.1016/j.nuclphysb.2010.07.015, doi:10.1016/ j.nuclphysb.2010.07.015.

[58] L. de Broglie,. Recherches sur la théorie des quanta (Researches on the quantum theory). PhD thesis, Paris, 1924.

[59] Kyoko Makino and Martin Berz. Cosy infinity version 8. Nuclear Instruments and Methods in Physics Research Section A: Accelerators, Spectrometers, Detectors and Associated Equipment, 427(1):338 - 343, 1999. URL: http://www. sciencedirect.com/science/article/pii/S016890029801554X, doi:https://doi. org/10.1016/S0168-9002(98)01554-X. 
[60] M. Mazouz, A. Camsonne, C. Muñoz Camacho, C. Ferdi, G. Gavalian, E. Kuchina, M. Amarian, K. A. Aniol, M. Beaumel, H. Benaoum, and et al. Deeply virtual compton scattering off the neutron. Physical Review Letters, 99(24), Dec 2007. URL: http://dx. doi.org/10.1103/PhysRevLett.99.242501, doi:10.1103/physrevlett.99.242501.

[61] R. W. McAllister and R. Hofstadter. Elastic scattering of 188-mev electrons from the proton and the alpha particle. Phys. Rev., 102:851-856, May 1956. URL: https: //link.aps.org/doi/10.1103/PhysRev.102.851, doi:10.1103/PhysRev.102.851.

[62] G. Miller, E. D. Bloom, G. Buschhorn, D. H. Coward, H. DeStaebler, J. Drees, C. L. Jordan, L. W. Mo, R. E. Taylor, J. I. Friedman, G. C. Hartmann, H. W. Kendall, and R. Verdier. Inelastic Electron-Proton Scattering at Large Momentum Transfers and the Inelastic Structure Functions of the Proton. Phys. Rev. D, 5:528-544, Feb 1972. URL: https://link.aps.org/doi/10.1103/PhysRevD.5.528, doi:10.1103/ PhysRevD.5.528.

[63] L. W. Mo and Y. S. Tsai. Radiative corrections to elastic and inelastic ep and up scattering. Rev. Mod. Phys., 41:205-235, Jan 1969. URL: https://link.aps.org/ doi/10.1103/RevModPhys.41.205, doi:10.1103/RevModPhys.41.205.

[64] D. Müller, D. Robaschik, B. Geyer, F.-M. Dittes, and J. Hořejši. Wave functions, evolution equations and evolution kernels from light-ray operators of qcd. Fortschritte der Physik/Progress of Physics, 42(2):101-141, 1994. URL: http://dx.doi.org/10. 1002/prop.2190420202, doi:10.1002/prop. 2190420202.

[65] Amy Orsborn. Assessment of Hall A Vertical Drift Chamber Analysis Software Performance Through Monte Carlo Simulation. Hall A Technical Note 05-069, 2005.

[66] M. Baylac P. Adderley, T.Day J. Clark, Hanskneckt J. Grames, J, and M. Stutzman M. Poelker. JEFFERSON LAB POLARIZED SOURCE, 2002. URL: https://www. jlab.org/accel/inj_group/docs/2002/ori.pdf.

[67] A.V Radyushkin. Scaling limit of deeply virtual compton scattering. Physics Letters B, 380(3-4):417-425, Jul 1996. URL: http://dx.doi.org/10.1016/0370-2693(96) 00528-X, doi:10.1016/0370-2693(96)00528-x.

[68] Michael Riordan. The discovery of quarks. Science, 256(5061):12871293, 1992. URL: https://science.sciencemag.org/content/256/5061/1287, 
arXiv:https://science.sciencemag.org/content/256/5061/1287.full.pdf, doi: $10.1126 /$ science.256.5061.1287.

[69] J. Roche, C. E. Hyde-Wright, B. Michel, and C. Munoz Camacho. Measurements of the Electron-Helicity Dependent Cross Sections of Deeply Virtual Compton Scattering with CEBAF at $12 \mathrm{GeV}, 2006$. arXiv:nucl-ex/0609015.

[70] Julie Roche. Private Communication.

[71] M. N. Rosenbluth. High energy elastic scattering of electrons on protons. Phys. Rev., 79:615-619, Aug 1950. URL: https://link.aps.org/doi/10.1103/PhysRev.79.615, doi:10.1103/PhysRev.79.615.

[72] E. Rutherford. The scattering of alpha and beta particles by matter and the structure of the atom. Phil. Mag. Ser. 6, 21:669-688, 1911. doi:10.1080/14786440508637080.

[73] Marat Rvachev. Effective use of JLab Hall A HRS acceptance with R-functions. Hall A Technical Note., 2005. URL: http://hallaweb.jlab.org/publications/Technotes/ files/2001/01-055.pdf.

[74] Alexa Stefanko. DIS Cross Section Extraction for E12-06-114. URL: https://www. jlab.org/indico/event/305/contribution/4/material/slides/0.pdf.

[75] Alexa Stefanko. Private Communication.

[76] Alexa Stefanko. R-Fuction Update. URL: https://hallaweb.jlab.org/dvcslog/12+ $\mathrm{GeV} / 435$.

[77] S. Stepanyan, V. D. Burkert, L. Elouadrhiri, G. S. Adams, E. Anciant, M. Anghinolfi, B. Asavapibhop, G. Audit, T. Auger, H. Avakian, and et al. Observation of exclusive deeply virtual compton scattering in polarized electron beam asymmetry measurements. Physical Review Letters, 87(18), Oct 2001. URL: http://dx.doi.org/10.1103/ PhysRevLett.87.182002, doi:10.1103/physrevlett.87.182002.

[78] Trigger - Hall A Wiki. URL: https://hallaweb.jlab.org/wiki/index.php/Trigger [cited 02/21/2019].

[79] M. Vanderhaeghen, J. M. Friedrich, D. Lhuillier, D. Marchand, L. Van Hoorebeke, and J. Van de Wiele. Qed radiative corrections to virtual compton scattering. Physical 
Review C, 62(2), Jul 2000. URL: http://dx.doi.org/10.1103/PhysRevC.62.025501, doi:10.1103/physrevc.62.025501

[80] G Zweig. An $\mathrm{SU}_{3}$ model for strong interaction symmetry and its breaking; Version 1. Technical Report CERN-TH-401, CERN, Geneva, Jan 1964. URL: https://cds.cern. ch/record/352337. 


\section{VITA}

Mohamed Nuhman Hashir Rashad

Department of Physics

Old Dominion University

Norfolk, VA 23529

Hashir Rashad was born in 1986 in Kalmunaikudy Sri Lanka, where he obtained his primary and secondary education. He attended Trinity College, Kandy for his advanced level education and obtained his Bachelors Degree in Physics from University of Peradeniya, Sri Lanka in 2009. Hashir joined Old Dominion University in August 2010 and obtained his Masters Degree in May, 2012. Following are few of his academic contributions and experiences during his $\mathrm{PhD}$ candidacy

\section{Publications}

M. Defurne et al. A glimpse of gluons through deeply virtual compton scattering on the proton. Nature Commun., 8(1):1408, 2017. arXiv:1703.09442, doi:10.1038/s41467-017-01819-3 M. Benali et al. Deeply virtual Compton scattering off the neutron. Nature Phys., 16(2):191-198, 2020. doi: 10.1038/s41567-019-0774-3

M. Defurne et al. Rosenbluth separation of the $\pi^{0}$ electroproduction cross section. Phys. Rev. Lett., 117(26):262001, 2016. arXiv:1608.01003,doi:10.1103/PhysRevLett.117.262001.

M. Mazouz et al. Rosenbluth separation of the $\pi^{0}$ Electroproduction Cross Section off the Neutron. Phys. Rev. Lett., 118(22):222002, 2017. doi:10.1103/PhysRevLett.118.222002

R. Cruz-Torres et al. Probing Few-Body Nuclear Dynamics via ${ }^{3} H$ and ${ }^{3} \mathrm{He}\left(e, e^{\prime} p\right) p n$ Cross-Section Measurements. Phys. Rev. Lett., 124(21):212501, 2020. doi:10.1103/PhysRevLett.124.212501

\section{Presentations}

Hall A Winter meeting : Deeply virtual Compton Scattering; January, 2019, JLab, VA, USA Joint Hall A and C summer meeting : DVCS; June, 2020, JLab, VA, USA

\section{Conferences and Summer Schools}

Hampton University Graduate School (HUGS); Summer 2012, JLab, VA, USA

National Nuclear Physics Summer School; Summer 2013, Stony Brook, NY

Photonuclear Reactions, Gordon Research Conference; August 2018, Holderness, NH 